\title{
Ruthenium-Catalyzed Highly Enantioselective Synthesis of Cis-3-Quinuclidinols via DKR Asymmetric Transfer Hydrogenation
}

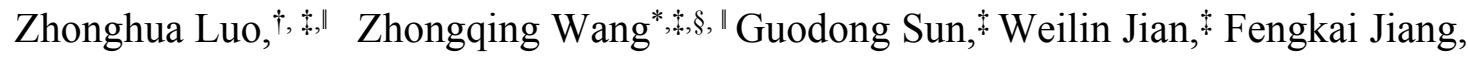
$\ddagger$ Baolei Luan, $₫$ Ridong Li, $\perp$ Lei Zhang, ${ }^{*}, \dagger$

'School of Biology and biological Engineering, South China University of Technology, Guangzhou 510640, P. R. China

*State Key Laboratory of Anti-Infective Drug Development, Sunshine Lake Pharma Co., Ltd, Dongguan 523871, P. R. China

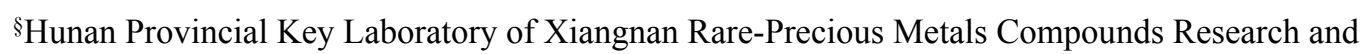
Application, Xiangnan University, Chenzhou 423000, China

${ }^{\perp}$ Institute of Systems Biomedicine, School of Basic Medical Sciences, Peking University Health Science Center, Beijing 100191, PR China

\section{Wangzhongqing@hec.cn; lzhangce@scut.edu.cn}

\section{Contents}

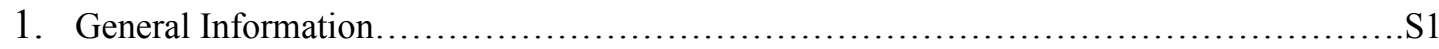

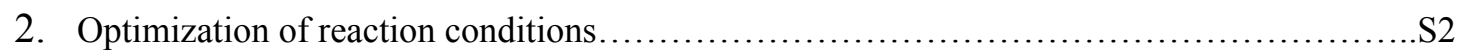

3. General procedure for the synthesis of 3-quinuclidinones $(\mathbf{1 a - w}) \ldots \ldots \ldots \ldots \ldots \ldots \ldots \ldots . . . . . . . \ldots \ldots$

4. General procedure for asymmetric transfer hydrogenation of 3-quinuclidinones (1a-w)......S12

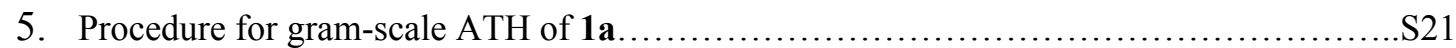

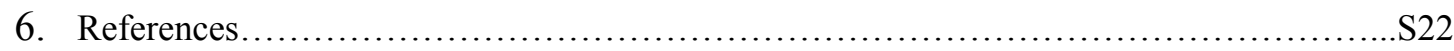

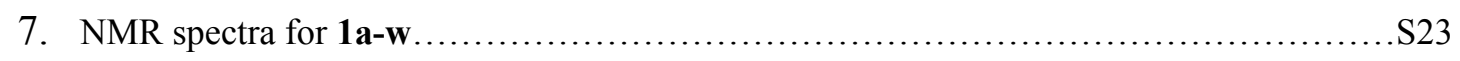

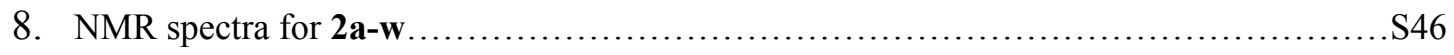

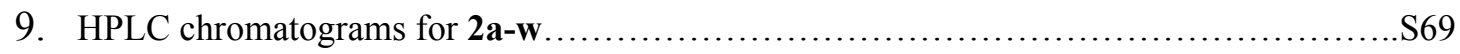




\section{General information}

Commercially available materials purchased from Alfa Aesar or Kelong (China) were used as received. Catalyst $(R, R)-\mathbf{C 4}[(\mathrm{R}, \mathrm{R})-\mathrm{Ts}-\mathrm{DENEB}]$ was purchased from Sino Compound Catalysts Co., Ltd. (China), and $(R, R)$-C6 was purchased from TCI (Shanghai) Development Co., Ltd. ( $R$, $R)-\mathbf{C 1},(R, R)-\mathbf{C 2},(R, R)-\mathbf{C 3}$, and $(R, R)-\mathbf{C 5}$ was synthesized in house. If no special treatments are indicated, all reagents and solvents were used without further purification. NMR spectra were measured on a Bruker Avance $400 \mathrm{MHz}$ spectrometer or $600 \mathrm{MHz}$ spectrometer in solvents indicated; chemical shifts are reported in units (ppm) by assigning the tetramethylsilane resonance in the ${ }^{1} \mathrm{H}$ spectrum as $0.00 \mathrm{ppm}$ and the $\mathrm{CDCl}_{3}$ resonance in the ${ }^{13} \mathrm{C}$ spectrum as $77.0 \mathrm{ppm}$ $\left[\right.$ DMSO- $\left.d_{6}(40.0 \mathrm{ppm})\right]$. Coupling constants are reported in hertz with multiplicities denoted as singlet (s), doublet (d), triplet (t), quartet (q), dd (doublet of doublets), m (multiplets), etc. High-resolution mass spectrometry (HRMS) was performed on a Fourier transform ion cyclotron resonance mass spectrometer. Analytical HPLC for the liquid phase was carried out on an Agilent HPLC workstation. Optical rotations were measured using a $1 \mathrm{~mL}$ cell with a $1 \mathrm{dm}$ path length on a Jasco P-1030 polarimeter and are reported as follows: $[\alpha]_{\mathrm{D}}^{26}(\mathrm{c}$ in g per $100 \mathrm{~mL}$ solvent).

\section{Optimization of reaction conditions}

Table S1. Screening of the solvents ${ }^{a}$

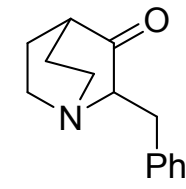

1a

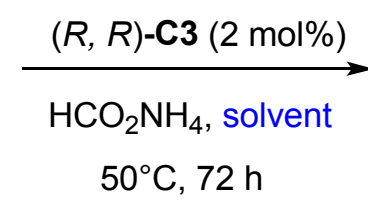

$50^{\circ} \mathrm{C}, 72 \mathrm{~h}$

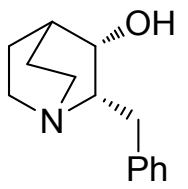

2a

\begin{tabular}{ccccc}
\hline entry & solvent & conv. $(\%)^{b}$ & $\mathrm{dr}^{c}$ & ee $(\%)^{c}$ \\
\hline 1 & DCM & trace & ND & ND \\
2 & EA & trace & ND & ND \\
3 & MeCN & 87 & $99: 1$ & 95 \\
4 & IPA & $>99$ & $>99: 1$ & 98 \\
5 & TFE & trace & ND & ND \\
6 & MeOH/DCM & $>99$ & $>99: 1$ & 97 \\
7 & IPA/DCM & $>99$ & $>99: 1$ & $>99$ \\
8 & IPA/DCE & $>99$ & $>99: 1$ & 97
\end{tabular}


${ }^{a}$ Conditions: $\mathbf{1 a}(0.5 \mathrm{mmol}), \mathrm{HCO}_{2} \mathrm{NH}_{4}\left(5.0\right.$ equiv), and catalyst $(R, R)-\mathbf{C 3}(2.0 \mathrm{~mol} \%)$ in $20 \mathrm{~mL}$ solvent at $50^{\circ} \mathrm{C}$ for 72 h. ${ }^{b}$ determined by HPLC; ${ }^{c}$ determined by HPLC using a Daicel Chiralpak IE column.

Table S2. Screening of reaction temperature and catalyst loading ${ }^{a}$<smiles>O=C1C2CCN(C2)C1Cc1ccccc1</smiles>

$1 \mathbf{a}$

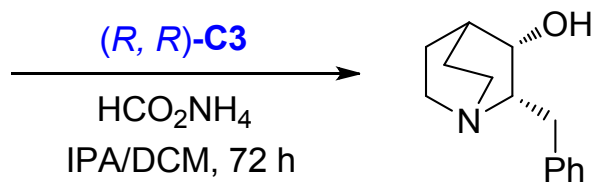

2a

\begin{tabular}{cccccc}
\hline entry & $\mathrm{T}\left({ }^{\circ} \mathrm{C}\right)$ & $\mathrm{S} / \mathrm{C}$ & conv. $(\%)^{b}$ & $\mathrm{dr}^{c}$ & ee $(\%)^{c}$ \\
\hline 1 & 40 & 50 & 90 & $>99: 1$ & $>99$ \\
2 & 50 & 50 & $>99$ & $>99: 1$ & $>99$ \\
3 & 60 & 50 & $>99$ & $>99: 1$ & 98 \\
4 & 50 & 100 & $>99$ & $>99: 1$ & $>99$ \\
5 & 60 & 100 & $>99$ & $>99: 1$ & 98 \\
6 & 70 & 100 & $>99$ & $>99: 1$ & 98 \\
7 & 50 & 200 & 92 & $>99: 1$ & $>99$ \\
8 & 70 & 200 & 97 & $>99: 1$ & 98 \\
9 & 70 & 500 & 95 & $>99: 1$ & 96 \\
\hline
\end{tabular}

${ }^{a}$ Conditions: $\mathbf{1 a}(0.5 \mathrm{mmol}), \mathrm{HCO}_{2} \mathrm{NH}_{4}\left(5.0\right.$ equiv), and catalyst $(R, R)-\mathbf{C} 3$ in $20 \mathrm{~mL}$ solvent for $72 \mathrm{~h} .{ }^{b}$ determined by HPLC; ${ }^{c}$ determined by HPLC using a Daicel Chiralpak IE column.

Table S3. Screening of the equivalent of ammonium formate<smiles>O=C1C2CCN(C2)C1Cc1ccccc1</smiles>

$1 \mathrm{a}$

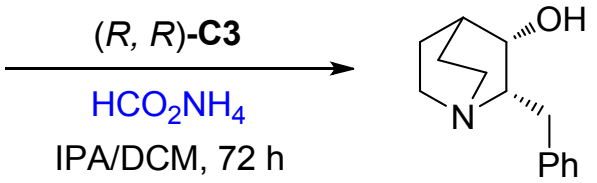

\begin{tabular}{ccccc}
\hline entry & $\mathrm{HCO}_{2} \mathrm{NH}_{4}$ & conv. $(\%)^{b}$ & $\mathrm{dr}^{c}$ & ee (\%) \\
\hline 1 & 4.0 equiv & 96 & $>99: 1$ & $>99$ \\
2 & 5.0 equiv & $>99$ & $>99: 1$ & $>99$ \\
4 & 6.0 equiv & $>99$ & $>99: 1$ & $>99$ \\
\hline
\end{tabular}

${ }^{a}$ Conditions: 1a $(0.5 \mathrm{mmol}), \mathrm{HCO}_{2} \mathrm{NH}_{4}$, and $(R, R)-\mathbf{C 3}(1.0 \mathrm{~mol} \%)$ in IPA/DCM $(20 \mathrm{~mL}, 1: 1)$ at $50^{\circ} \mathrm{C}$ for $72 \mathrm{~h}$. ${ }^{b}$ determined by HPLC; ${ }^{c}$ determined by HPLC using a Daicel Chiralpak IE column. 


\section{General procedure for the synthesis of 3-quinuclidinones (1a-w)}

3-Quinuclidinones 1a-s and 1t-w were synthesized via Method A and Method B, respectively, according to the previously reported procedures ${ }^{1,2}$. Among them, $1 \mathrm{~d}, 1 \mathbf{e}, \mathbf{1 h}, \mathbf{1 k}, \mathbf{1 l}, \mathbf{1 n}, \mathbf{1 0}, 1 \mathbf{s}, 1 \mathbf{w}$ are new compounds.

\subsection{Preparation of 3-quinuclidinones (1a-s)}

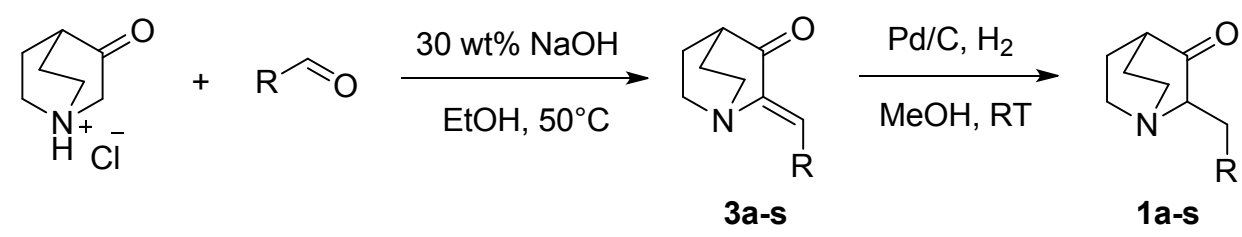

Method A: To a solution of quinuclidin-3-one hydrochloride $(15 \mathrm{mmol})$ in EtOH $(20 \mathrm{~mL})$ was added $\mathrm{NaOH}$ aqueous solution (30 wt $\%, 21 \mathrm{mmol})$, The mixture was stirred at room temperature for $30 \mathrm{~min}$; then benzaldehyde $(15 \mathrm{mmol})$ was added in one portion. The resulting solution was heated in an oil bath at $50^{\circ} \mathrm{C}$ for $8 \mathrm{~h}$, and diluted with $30 \mathrm{~mL}$ of water. The product was collected by filtration and washed with $\mathrm{EtOH} / \mathrm{H}_{2} \mathrm{O}(1: 1,2 \times 20 \mathrm{~mL})$ to afford the product 3 as a yellow solid.

MeOH $(20 \mathrm{~mL})$ was added to the above obtained $3(10 \mathrm{mmol})$. Activated $\mathrm{Pd} / \mathrm{C}(5 \mathrm{wt} \%)$ was charged, and the mixture was stirred under a $\mathrm{H}_{2}$ atmosphere $(1 \mathrm{~atm})$ at room temperature for $4 \mathrm{~h}$. The mixture was filtered to remove $\mathrm{Pd} / \mathrm{C}$ and washed with $\mathrm{MeOH}(10 \mathrm{~mL})$. The filtrate was concentrated under vacuum and the crude product was purified by flash chromatography on silica gel (petroleum ether/ethyl acetate $=5: 1$ ) afforded the product $\mathbf{1}$ as a white solid.

\subsection{Preparation of 3-quinuclidinones (1t-w)}

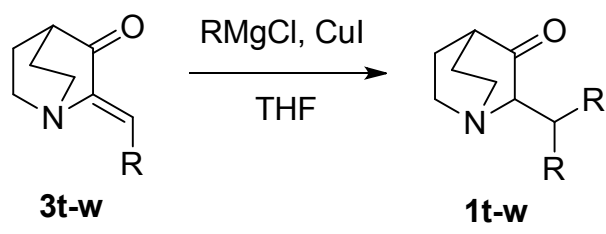

Method B: To a mixture of the corresponding Grignard reagent $(10 \mathrm{mmol})$ and $\mathrm{CuI}(76.2 \mathrm{mg}, 0.4$ $\mathrm{mmol})$ in $20 \mathrm{~mL}$ of THF, was added dropwise a solution of $\mathbf{3}(8.0 \mathrm{mmol})$ in $20 \mathrm{~mL}$ of THF at $0{ }^{\circ} \mathrm{C}$. The mixture was stirred at $0{ }^{\circ} \mathrm{C}$ for 30 minutes then at room temperature for $1-2 \mathrm{~h}$. The reaction mixture was quenched with water $(30 \mathrm{~mL})$, and extracted with $\mathrm{CH}_{2} \mathrm{Cl}_{2}(2 \times 30 \mathrm{~mL})$. The combined organic layers were washed with saturated $\mathrm{NaCl}$ solution and dried over $\mathrm{Na}_{2} \mathrm{SO}_{4}$, and concentrated under reduced pressure. The crude product was purified by flash chromatography on 
silica gel (petroleum ether/ethyl acetate $=10 / 1$ to $8 / 2$ ) to give the desired product 1 .

2-Benzylquinuclidin-3-one (1a) ${ }^{3}$ :<smiles>O=C1C2CCN(C2)C1Cc1ccccc1</smiles>

white solid, $2.5 \mathrm{~g}, 78 \%$ yield. ${ }^{1} \mathrm{H}$ NMR $\left(400 \mathrm{MHz}, \mathrm{CDCl}_{3}\right) \delta 7.36-7.26(\mathrm{~m}, 4 \mathrm{H})$, $7.25-7.19(\mathrm{~m}, 1 \mathrm{H}), 3.41(\mathrm{dd}, J=10.7,3.8 \mathrm{~Hz}, 1 \mathrm{H}), 3.29-3.18(\mathrm{~m}, 2 \mathrm{H}), 3.18-$ $3.06(\mathrm{~m}, 1 \mathrm{H}), 2.96-2.85(\mathrm{~m}, 2 \mathrm{H}), 2.80(\mathrm{dd}, J=14.8,10.8 \mathrm{~Hz}, 1 \mathrm{H}), 2.49$ (p, $J=2.9$ $\mathrm{Hz}, 1 \mathrm{H}), 2.11-1.90(\mathrm{~m}, 4 \mathrm{H}) .{ }^{13} \mathrm{C} \mathrm{NMR}\left(101 \mathrm{MHz}, \mathrm{CDCl}_{3}\right) \delta 221.1,139.1$, 128.7, 1a $128.5,126.4,71.5,48.9,41.0,40.1,33.7,26.7,25.2$.

2-(4-Methoxybenzyl)quinuclidin-3-one (1b) ${ }^{4}$ :

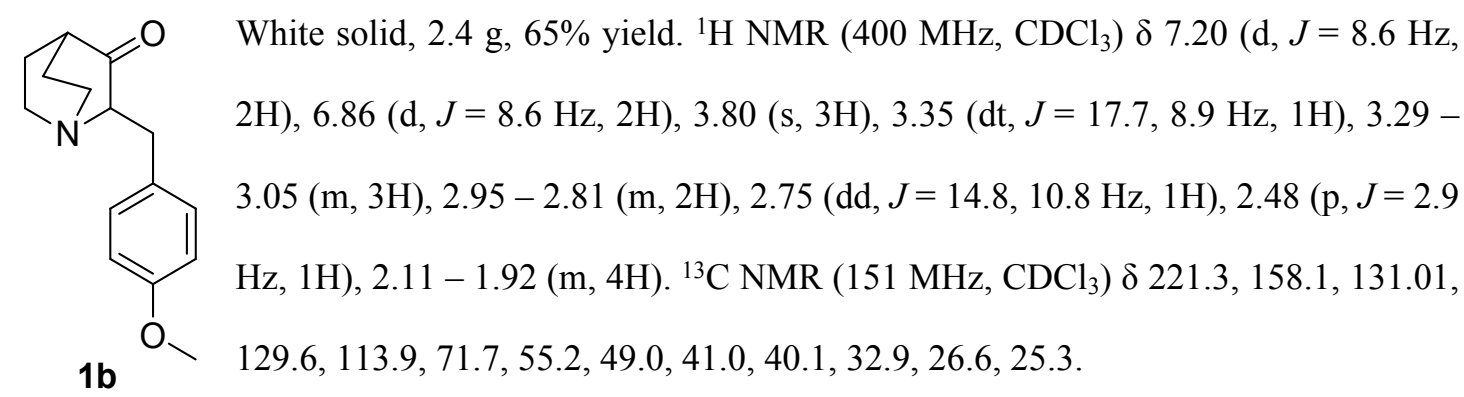

2-(3-Methylbenzyl)quinuclidin-3-one (1c) ${ }^{5}$ :<smiles>Cc1cccc(CC2C(=O)C3CCN2CC3)c1</smiles>

$1 \mathrm{c}$

White solid, $2.6 \mathrm{~g}, 76 \%$ yield. ${ }^{1} \mathrm{H}$ NMR $\left(400 \mathrm{MHz}, \mathrm{CDCl}_{3}\right) \delta 7.21(\mathrm{t}, J=7.9 \mathrm{~Hz}$, 1H), $7.06(\mathrm{dd}, J=15.1,7.1 \mathrm{~Hz}, 3 \mathrm{H}), 3.42(\mathrm{dd}, J=10.7,3.8 \mathrm{~Hz}, 1 \mathrm{H}), 3.29-3.07$ (m, 3H), $2.96-2.83(\mathrm{~m}, 2 \mathrm{H}), 2.76(\mathrm{dd}, J=14.7,10.8 \mathrm{~Hz}, 1 \mathrm{H}), 2.52-2.45(\mathrm{~m}$, 1H), 2.35 (s, 3H), 2.01 (ddd, $J=10.4,8.6,2.9 \mathrm{~Hz}, 4 \mathrm{H}) .{ }^{13} \mathrm{C}$ NMR $(151 \mathrm{MHz}$, $\left.\mathrm{CDCl}_{3}\right) \delta 221.3,139.0,138.0,129.5,128.4,127.2,125.7,71.6,49.0,41.0,40.1$, 33.7, 26.6, 25.2, 21.5.

\section{2-(3,4-Dimethylbenzyl)quinuclidin-3-one (1d):}<smiles>Cc1ccc(CC2C(=O)C3CCN2CC3)cc1C</smiles>

White solid, $2.9 \mathrm{~g}, 81 \%$ yield. ${ }^{1} \mathrm{H}$ NMR $\left(400 \mathrm{MHz}, \mathrm{CDCl}_{3}\right) \delta 7.08(\mathrm{~d}, J=7.6$ $\mathrm{Hz}, 1 \mathrm{H}), 7.06-6.97(\mathrm{~m}, 2 \mathrm{H}), 3.41(\mathrm{dd}, J=10.9,3.9 \mathrm{~Hz}, 1 \mathrm{H}), 3.30-3.04(\mathrm{~m}$, $3 \mathrm{H}), 2.88(\mathrm{qd}, J=10.5,6.9 \mathrm{~Hz}, 2 \mathrm{H}), 2.72(\mathrm{dd}, J=14.7,10.9 \mathrm{~Hz}, 1 \mathrm{H}), 2.48(\mathrm{p}, J$ $=2.9 \mathrm{~Hz}, 1 \mathrm{H}), 2.25(\mathrm{~d}, J=9.1 \mathrm{~Hz}, 6 \mathrm{H}), 2.11-1.92(\mathrm{~m}, 4 \mathrm{H}) .{ }^{13} \mathrm{C}$ NMR $(151$ $\left.\mathrm{MHz}, \mathrm{CDCl}_{3}\right) \delta 221.4,136.5,136.3,134.6,130.0,129.8,126.0,71.7,49.0,41.0$, 40.1, 33.5, 26.5, 25.3, 19.9, 19.4. HRMS (ESI-TOF) m/z: $[\mathrm{M}+\mathrm{H}]^{+}$Calcd. for $\left(\mathrm{C}_{16} \mathrm{H}_{22} \mathrm{NO}\right)$ 


\section{N-(3-((3-oxoquinuclidin-2-yl)methyl)phenyl)acetamide (1e):}

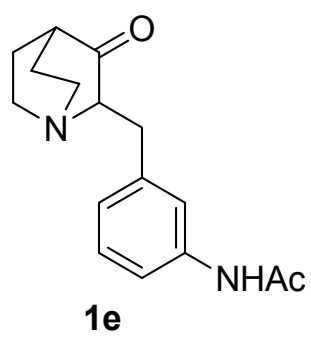

White solid, $2.5 \mathrm{~g}, 61 \%$ yield. ${ }^{1} \mathrm{H}$ NMR (400 MHz, $\left.\mathrm{CDCl}_{3}\right) \delta 7.44(\mathrm{~d}, J=$ $8.4 \mathrm{~Hz}, 2 \mathrm{H}), 7.34$ (d, $J=8.0 \mathrm{~Hz}, 1 \mathrm{H}), 7.24(\mathrm{t}, J=7.8 \mathrm{~Hz}, 1 \mathrm{H}), 7.01$ (d, $J=$ $7.4 \mathrm{~Hz}, 1 \mathrm{H}), 3.40(\mathrm{dd}, J=10.7,3.5 \mathrm{~Hz}, 1 \mathrm{H}), 3.21(\mathrm{dd}, J=14.8,3.8 \mathrm{~Hz}$, 2H), $3.11(\mathrm{dt}, J=15.3,7.9 \mathrm{~Hz}, 1 \mathrm{H}), 2.88(\mathrm{tt}, J=14.5,7.1 \mathrm{~Hz}, 2 \mathrm{H}), 2.77$ (dd, $J=14.7,10.9 \mathrm{~Hz}, 1 \mathrm{H}), 2.53-2.41(\mathrm{~m}, 1 \mathrm{H}), 2.14(\mathrm{~s}, 3 \mathrm{H}), 2.08-1.95$

(m, 4H). ${ }^{13} \mathrm{C}$ NMR $\left(101 \mathrm{MHz}, \mathrm{CDCl}_{3}\right) \delta 221.0,168.3,140.0,138.1,129.0,124.6,120.1,118.0$, 71.4, 48.9, 41.0, 40.0, 33.6, 26.7, 25.1, 24.6. HRMS (ESI-TOF) $\mathrm{m} / \mathrm{z}:[\mathrm{M}+\mathrm{H}]^{+}$Calcd. for $\left(\mathrm{C}_{16} \mathrm{H}_{21} \mathrm{~N}_{2} \mathrm{O}_{2}\right)$ 273.1603; Found 273.1594.

\section{2-(2-Hydroxybenzyl)quinuclidin-3-one (1f) ${ }^{6}$ :}<smiles>O=C1C2CCN(C2)C1Cc1ccccc1O</smiles>

$1 f$

White solid, $1.5 \mathrm{~g}, 43 \%$ yield. ${ }^{1} \mathrm{H}$ NMR $\left(400 \mathrm{MHz}, \mathrm{CDCl}_{3}\right) \delta 7.14$ (dd, $J=$ 16.5, 7.6 Hz, 2H), 6.87 (d, $J=7.9 \mathrm{~Hz}, 1 \mathrm{H}), 6.78$ (t, $J=7.4 \mathrm{~Hz}, 1 \mathrm{H}), 3.51-$ $3.31(\mathrm{~m}, 2 \mathrm{H}), 3.19(\mathrm{dd}, J=11.2,5.2 \mathrm{~Hz}, 2 \mathrm{H}), 3.12(\mathrm{~d}, J=5.9 \mathrm{~Hz}, 2 \mathrm{H}), 3.03$ (dt, $J=14.4,7.4 \mathrm{~Hz}, 1 \mathrm{H}), 2.66-2.50(\mathrm{~m}, 1 \mathrm{H}), 2.14(\mathrm{td}, J=8.2,2.7 \mathrm{~Hz}, 2 \mathrm{H})$, $2.09-2.01(\mathrm{~m}, 2 \mathrm{H}) .{ }^{13} \mathrm{C}$ NMR $\left(101 \mathrm{MHz}, \mathrm{CDCl}_{3}\right) \delta 216.2,157.3,131.2$, $128.6,125.2,119.3,117.9,71.3,47.7,41.2,39.3,30.0,27.1,23.3$.

2-(2-Fluorobenzyl)quinuclidin-3-one (1g) ${ }^{7}$ :<smiles>O=C1C2CCN(C2)C1Cc1ccccc1F</smiles>

$1 \mathrm{~g}$

White solid, $2.4 \mathrm{~g}, 69 \%$ yield. ${ }^{1} \mathrm{H}$ NMR $\left(400 \mathrm{MHz}, \mathrm{CDCl}_{3}\right) \delta 7.28(\mathrm{dd}, J=4.7$, $2.6 \mathrm{~Hz}, 1 \mathrm{H}), 7.24-7.17(\mathrm{~m}, 1 \mathrm{H}), 7.14-6.98(\mathrm{~m}, 2 \mathrm{H}), 3.46(\mathrm{dd}, J=11.0,4.1$ $\mathrm{Hz}, 1 \mathrm{H}), 3.32-3.19(\mathrm{~m}, 2 \mathrm{H}), 3.11(\mathrm{dt}, J=15.5,7.9 \mathrm{~Hz}, 1 \mathrm{H}), 2.96-2.79(\mathrm{~m}$, $3 \mathrm{H}), 2.54-2.44(\mathrm{~m}, 1 \mathrm{H}), 2.11-1.93(\mathrm{~m}, 4 \mathrm{H}) .{ }^{13} \mathrm{C} \mathrm{NMR}\left(151 \mathrm{MHz}, \mathrm{CDCl}_{3}\right) \delta$ $220.8,162.1,160.5,130.9,130.8,128.1,128.1,125.9,125.8,124.1,124.1$, $115.4,115.3,70.3,70.3,48.9,41.0,40.0,27.1,27.1,26.7,25.0$.

2-(3-Fluorobenzyl)quinuclidin-3-one (1h): 


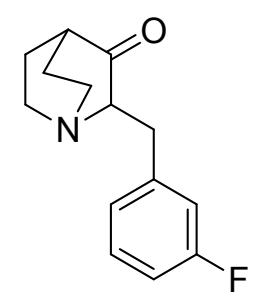

$1 \mathrm{~h}$

White solid, $2.5 \mathrm{~g}, 73 \%$ yield. ${ }^{1} \mathrm{H}$ NMR (400 MHz, $\left.\mathrm{CDCl}_{3}\right) \delta 7.32-7.25(\mathrm{~m}$, 1H), $7.03(\mathrm{dd}, J=15.5,8.8 \mathrm{~Hz}, 2 \mathrm{H}), 6.92(\mathrm{td}, J=8.4,2.0 \mathrm{~Hz}, 1 \mathrm{H}), 3.38(\mathrm{dd}, J=$ 10.8, 3.7 Hz, 1H), $3.28-3.04(\mathrm{~m}, 3 \mathrm{H}), 2.99-2.84(\mathrm{~m}, 2 \mathrm{H}), 2.79(\mathrm{dd}, J=14.9$,

$10.9 \mathrm{~Hz}, 1 \mathrm{H}), 2.55-2.42(\mathrm{~m}, 1 \mathrm{H}), 2.03(\mathrm{tdd}, J=10.3,7.5,3.4 \mathrm{~Hz}, 4 \mathrm{H}) .{ }^{13} \mathrm{C}$ NMR $\left(151 \mathrm{MHz}, \mathrm{CDCl}_{3}\right) \delta 220.9,163.7,162.1,141.7,141.6,129.9,129.8$, $124.4,124.4,115.7,115.6,113.4,113.3,71.2,48.9,41.0,40.00,33.3,33.2,26.8,25.0$. HRMS (ESI-TOF) m/z: $[\mathrm{M}+\mathrm{H}]^{+}$Calcd. for $\left(\mathrm{C}_{14} \mathrm{H}_{17} \mathrm{FNO}\right)$ 234.1294; Found 234.1283.

2-([1,1'-Biphenyl]-4-ylmethyl)quinuclidin-3-one (1i) ${ }^{8}$ :<smiles>O=C1C2CCN(C2)C1Cc1ccc(-c2ccccc2)cc1</smiles>

White solid, $3.7 \mathrm{~g}, 84 \%$ yield. ${ }^{1} \mathrm{H}$ NMR (400 MHz, $\left.\mathrm{CDCl}_{3}\right) \delta 7.64-7.50(\mathrm{~m}, 4 \mathrm{H})$, $7.44(\mathrm{t}, J=7.6 \mathrm{~Hz}, 2 \mathrm{H}), 7.35(\mathrm{t}, J=8.7 \mathrm{~Hz}, 3 \mathrm{H}), 3.46(\mathrm{dd}, J=10.8,3.8 \mathrm{~Hz}, 1 \mathrm{H})$, $3.35-3.22(\mathrm{~m}, 2 \mathrm{H}), 3.16(\mathrm{dt}, J=15.4,7.8 \mathrm{~Hz}, 1 \mathrm{H}), 2.99-2.79(\mathrm{~m}, 3 \mathrm{H}), 2.51(\mathrm{dd}$, $J=5.9,2.9 \mathrm{~Hz}, 1 \mathrm{H}), 2.16-1.94(\mathrm{~m}, 4 \mathrm{H}) .{ }^{13} \mathrm{C} \mathrm{NMR}\left(101 \mathrm{MHz}, \mathrm{CDCl}_{3}\right) \delta 221.1$, $1 \mathrm{i}$ $141.1,139.3,138.2,129.1,128.7,127.3,127.0,71.5,49.0,41.0,40.1,33.4,26.7$, 25.2

\section{2-(4-Fluorobenzyl)quinuclidin-3-one (1j) ${ }^{4}$ :}<smiles>O=C1C2CCN(C2)C1Cc1ccc(F)cc1</smiles>

White solid, $2.6 \mathrm{~g}, 76 \%$ yield. ${ }^{1} \mathrm{H}$ NMR $\left(400 \mathrm{MHz}, \mathrm{CDCl}_{3}\right) \delta 7.24(\mathrm{dd}, J=8.4,5.5$ Hz, 2H), 6.99 (dd, $J=12.1,5.3 \mathrm{~Hz}, 2 \mathrm{H}), 3.34$ (dd, $J=10.7,3.9 \mathrm{~Hz}, 1 \mathrm{H}), 3.26-$ $3.02(\mathrm{~m}, 3 \mathrm{H}), 2.88(\mathrm{ddd}, J=9.4,7.5,4.0 \mathrm{~Hz}, 2 \mathrm{H}), 2.77(\mathrm{dd}, J=14.9,10.8 \mathrm{~Hz}, 1 \mathrm{H})$, $2.49(\mathrm{p}, J=2.9 \mathrm{~Hz}, 1 \mathrm{H}), 2.13-1.95(\mathrm{~m}, 4 \mathrm{H}) .{ }^{13} \mathrm{C} \mathrm{NMR}\left(101 \mathrm{MHz}, \mathrm{CDCl}_{3}\right) \delta$ $220.9,162.8,160.3,134.7,134.7,130.2,130.1,115.3,115.1,71.5,48.9,41.0,40.0$, $32.8,26.7,25.1$.

\section{2-(4-(Trifluoromethyl)benzyl)quinuclidin-3-one (1k):}<smiles>O=C1C2CCN(C2)C1Cc1ccc(C(F)(F)F)cc1</smiles>

White solid, $3.1 \mathrm{~g}, 73 \%$ yield. ${ }^{1} \mathrm{H}$ NMR $\left(400 \mathrm{MHz}, \mathrm{CDCl}_{3}\right) \delta 7.57(\mathrm{~d}, J=8.0 \mathrm{~Hz}$, 2H), $7.40(\mathrm{~d}, J=8.0 \mathrm{~Hz}, 2 \mathrm{H}), 3.39(\mathrm{dd}, J=10.8,3.8 \mathrm{~Hz}, 1 \mathrm{H}), 3.28(\mathrm{dd}, J=14.9$, $3.8 \mathrm{~Hz}, 1 \mathrm{H}), 3.25-3.04(\mathrm{~m}, 2 \mathrm{H}), 2.98-2.78(\mathrm{~m}, 3 \mathrm{H}), 2.57-2.46(\mathrm{~m}, 1 \mathrm{H}), 2.16-$ $1.92(\mathrm{~m}, 4 \mathrm{H}) .{ }^{13} \mathrm{C} \mathrm{NMR}\left(101 \mathrm{MHz}, \mathrm{CDCl}_{3}\right) \delta 220.6,143.3,129.1,128.8,128.5$, 
125.6, 125.4, 125.4, 125.4, 125.3, 122.9, 71.1, 48.9, 41.1, 34.0, 33.3, 26.9, 24.9. HRMS (ESI-TOF) $\mathrm{m} / \mathrm{z}:[\mathrm{M}+\mathrm{H}]^{+}$Calcd. for $\left(\mathrm{C}_{15} \mathrm{H}_{17} \mathrm{~F}_{3} \mathrm{NO}\right)$ 284.1262; Found 284.1253 .

\section{2-(2,5-Difluorobenzyl)quinuclidin-3-one (11):}

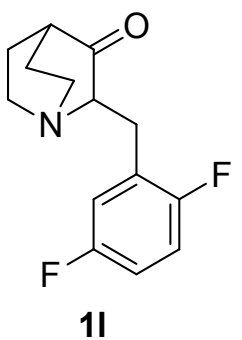

White solid, $2.2 \mathrm{~g}, 60 \%$ yield. ${ }^{1} \mathrm{H}$ NMR $\left(400 \mathrm{MHz}, \mathrm{CDCl}_{3}\right) \delta 7.05-6.94(\mathrm{~m}$, 2H), $6.92-6.83(\mathrm{~m}, 1 \mathrm{H}), 3.43(\mathrm{dd}, J=11.1,4.0 \mathrm{~Hz}, 1 \mathrm{H}), 3.30-3.05(\mathrm{~m}, 3 \mathrm{H})$, $2.99-2.71(\mathrm{~m}, 3 \mathrm{H}), 2.54-2.41(\mathrm{~m}, 1 \mathrm{H}), 2.07-2.00(\mathrm{~m}, 3 \mathrm{H}), 1.67(\mathrm{~s}, 1 \mathrm{H}) .{ }^{13} \mathrm{C}$ NMR $\left(151 \mathrm{MHz}, \mathrm{CDCl}_{3}\right) \delta 220.41,159.4,159.4,158.0,158.0,157.8,157.8$, $156.5,156.4,127.7,127.7,127.6,127.6,117.3,117.2,117.1,117.1,116.3$, 116.3, 116.1, 116.1, 114.5, 114.4, 114.3, 114.3, 69.9, 69.9, 48.9, 41.0, 39.9, 26.9, 24.9. HRMS (ESI-TOF) m/z: [M+H $]^{+}$Calcd. for $\left(\mathrm{C}_{14} \mathrm{H}_{16} \mathrm{~F}_{2} \mathrm{NO}\right)$ 252.1200; Found 252.1190 .

\section{2-(3,5-Dimethoxybenzyl)quinuclidin-3-one (1m) ${ }^{9}$ :}

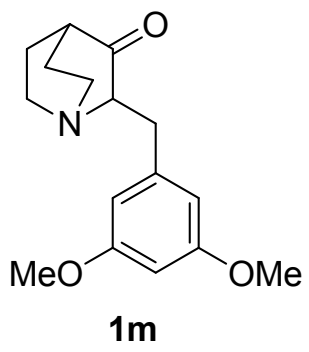

White solid, $3.3 \mathrm{~g}, 82 \%$ yield. ${ }^{1} \mathrm{H}$ NMR (400 MHz, $\left.\mathrm{CDCl}_{3}\right) \delta 6.45(\mathrm{~d}, J=$ $2.1 \mathrm{~Hz}, 2 \mathrm{H}), 6.33$ (t, $J=2.1 \mathrm{~Hz}, 1 \mathrm{H}), 3.79(\mathrm{~s}, 6 \mathrm{H}), 3.42(\mathrm{dd}, J=10.7,3.7$ $\mathrm{Hz}, 1 \mathrm{H}), 3.28-3.06(\mathrm{~m}, 3 \mathrm{H}), 2.99-2.81$ (m, 2H), 2.73 (dd, $J=14.7,10.8$ $\mathrm{Hz}, 1 \mathrm{H}), 2.48(\mathrm{dd}, J=5.9,2.9 \mathrm{~Hz}, 1 \mathrm{H}), 2.02(\mathrm{tdd}, J=10.2,7.4,3.2 \mathrm{~Hz}$, 4H). ${ }^{13} \mathrm{C}$ NMR $\left(101 \mathrm{MHz}, \mathrm{CDCl}_{3}\right) \delta 221.2,160.8,141.4,106.9,98.3,71.4$, $55.2,49.0,41.0,40.1,34.0,26.7,25.2$.

\section{2-(3-Hydroxy-4-Methoxybenzyl)quinuclidin-3-one (1n):}

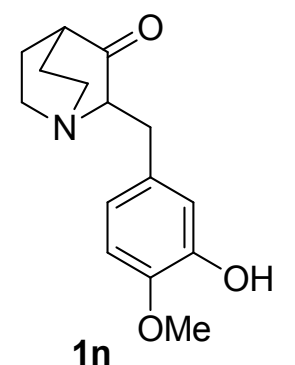

White solid, $2.6 \mathrm{~g}, 62 \%$ yield. ${ }^{1} \mathrm{H}$ NMR $\left(400 \mathrm{MHz}, \mathrm{CDCl}_{3}\right) \delta 6.81(\mathrm{~d}, J=1.7$ $\mathrm{Hz}, 1 \mathrm{H}), 6.73$ (dt, $J=8.2,5.0 \mathrm{~Hz}, 2 \mathrm{H}), 6.04$ (s, 1H), 3.84 (s, 3H), 3.38 (dd, $J$ $=11.0,3.8 \mathrm{~Hz}, 1 \mathrm{H}), 3.31-3.04(\mathrm{~m}, 3 \mathrm{H}), 2.96-2.80(\mathrm{~m}, 2 \mathrm{H}), 2.69(\mathrm{dd}, J=$ $14.8,11.0 \mathrm{~Hz}, 1 \mathrm{H}), 2.48(\mathrm{dd}, J=5.9,2.9 \mathrm{~Hz}, 1 \mathrm{H}), 2.12-1.89(\mathrm{~m}, 4 \mathrm{H}) .{ }^{13} \mathrm{C}$ NMR (101 MHz, $\left.\mathrm{CDCl}_{3}\right) \delta 221.0,145.6,145.3,132.0,112.0,115.1,110.7$, 71.6, 55.8, 48.9, 40.9, 40.1, 33.2, 26.4, 25.2. Found 262.1435. HRMS (ESI-TOF) m/z: $[\mathrm{M}+\mathrm{H}]^{+}$ Calcd. for $\left(\mathrm{C}_{15} \mathrm{H}_{20} \mathrm{FNO}_{3}\right)$ 262.1443; Found 262.1435. 


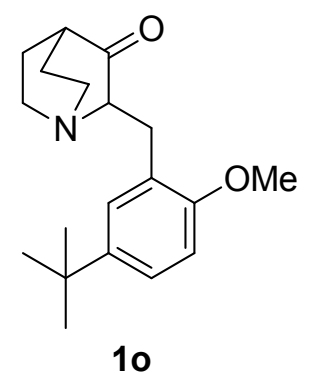

White solid, $3.5 \mathrm{~g}, 77 \%$ yield. ${ }^{1} \mathrm{H}$ NMR (400 $\left.\mathrm{MHz}, \mathrm{CDCl}_{3}\right) \delta 7.26-7.17$ $(\mathrm{m}, 2 \mathrm{H}), 6.80(\mathrm{~d}, J=8.9 \mathrm{~Hz}, 1 \mathrm{H}), 3.83(\mathrm{~s}, 3 \mathrm{H}), 3.60(\mathrm{dd}, J=10.5,4.8 \mathrm{~Hz}$, 1H), $3.41-3.28(\mathrm{~m}, 1 \mathrm{H}), 3.22(\mathrm{dd}, J=14.4,4.9 \mathrm{~Hz}, 1 \mathrm{H}), 3.15-3.00(\mathrm{~m}$, 1H), $2.95-2.76(\mathrm{~m}, 3 \mathrm{H}), 2.46(\mathrm{dd}, J=5.9,2.9 \mathrm{~Hz}, 1 \mathrm{H}), 2.02(\mathrm{ddd}, J=20.5$, 11.5, $5.4 \mathrm{~Hz}, 4 \mathrm{H}), 1.31(\mathrm{~s}, 9 \mathrm{H}) .{ }^{13} \mathrm{C} \mathrm{NMR}\left(101 \mathrm{MHz}, \mathrm{CDCl}_{3}\right) \delta 221.8,155.5$, $142.8,127.8,126.3,124.2,109.9,70.1,55.3,49.0,40.9,40.3,34.0,31.5$, 29.5, 26.4, 25.5. HRMS (ESI-TOF) $\mathrm{m} / \mathrm{z}$ : $[\mathrm{M}+\mathrm{H}]^{+}$Calcd. for $\left(\mathrm{C}_{19} \mathrm{H}_{28} \mathrm{NO}_{2}\right)$ 302.2120; Found 302.2113

2-(Benzo[d][1,3]dioxol-5-ylmethyl)quinuclidin-3-one $(1 p){ }^{10}$ :

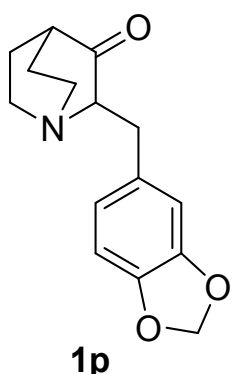

White solid, $2.8 \mathrm{~g}, 72 \%$ yield. ${ }^{1} \mathrm{H}$ NMR (400 MHz, $\left.\mathrm{CDCl}_{3}\right) \delta 6.79(\mathrm{~d}, J=1.1$ $\mathrm{Hz}, 1 \mathrm{H}), 6.73$ (q, $J=7.9 \mathrm{~Hz}, 2 \mathrm{H}), 5.93(\mathrm{~s}, 2 \mathrm{H}), 3.34$ (dd, $J=10.8,3.8 \mathrm{~Hz}, 1 \mathrm{H})$, $3.24-3.08(\mathrm{~m}, 3 \mathrm{H}), 2.96-2.79(\mathrm{~m}, 2 \mathrm{H}), 2.71(\mathrm{dd}, J=14.9,10.9 \mathrm{~Hz}, 1 \mathrm{H}), 2.48$ $(\mathrm{p}, J=2.9 \mathrm{~Hz}, 1 \mathrm{H}), 2.02(\mathrm{tdd}, J=10.2,7.7,3.6 \mathrm{~Hz}, 4 \mathrm{H}) .{ }^{13} \mathrm{C}$ NMR $(151 \mathrm{MHz}$, $\left.\mathrm{CDCl}_{3}\right) \delta 221.1,147.7,146.1,132.7,121.6,109.1,108.3,100.9,71.7,49.0$, $41.0,40.1,33.4,26.6,25.2$.

2-(Naphthalen-1-ylmethyl)quinuclidin-3-one (1q) ${ }^{11}$ :<smiles>O=C1C2CCN(C2)C1Cc1cccc2ccccc12</smiles>

19

White solid, $3.5 \mathrm{~g}, 88 \%$ yield. ${ }^{1} \mathrm{H}$ NMR $\left(400 \mathrm{MHz}, \mathrm{CDCl}_{3}\right) \delta 8.18(\mathrm{~d}, J=8.3$ Hz, 1H), 7.89 (d, $J=7.8 \mathrm{~Hz}, 1 \mathrm{H}), 7.76(\mathrm{dd}, J=6.0,3.3 \mathrm{~Hz}, 1 \mathrm{H}), 7.62-7.47$ (m, 2H), $7.44(\mathrm{dd}, J=7.9,5.2 \mathrm{~Hz}, 2 \mathrm{H}), 3.80(\mathrm{dd}, J=14.9,3.6 \mathrm{~Hz}, 1 \mathrm{H}), 3.56$ $(\mathrm{dd}, J=9.7,3.3 \mathrm{~Hz}, 1 \mathrm{H}), 3.37(\mathrm{ddd}, J=10.4,8.7,2.4 \mathrm{~Hz}, 1 \mathrm{H}), 3.24-3.05$ $(\mathrm{m}, 2 \mathrm{H}), 3.03-2.90(\mathrm{~m}, 1 \mathrm{H}), 2.85(\mathrm{ddd}, J=8.9,7.7,2.4 \mathrm{~Hz}, 1 \mathrm{H}), 2.60-2.47$ (m, 1H), $2.10(\mathrm{td}, J=8.2,2.7 \mathrm{~Hz}, 2 \mathrm{H}), 1.99$ (td, $J=6.6,3.1 \mathrm{~Hz}, 2 \mathrm{H}) .{ }^{13} \mathrm{C}$ NMR $(101 \mathrm{MHz}$, $\left.\mathrm{CDCl}_{3}\right) \delta 221.3,135.0,134.0,131.7,129.0,127.2,127.1,126.1,125.5,125.5,123.5,70.7,48.9$, $41.3,40.1,31.1,27.0,25.0$.

\section{2-(Furan-2-ylmethyl)quinuclidin-3-one (1r) ${ }^{4}$ :}

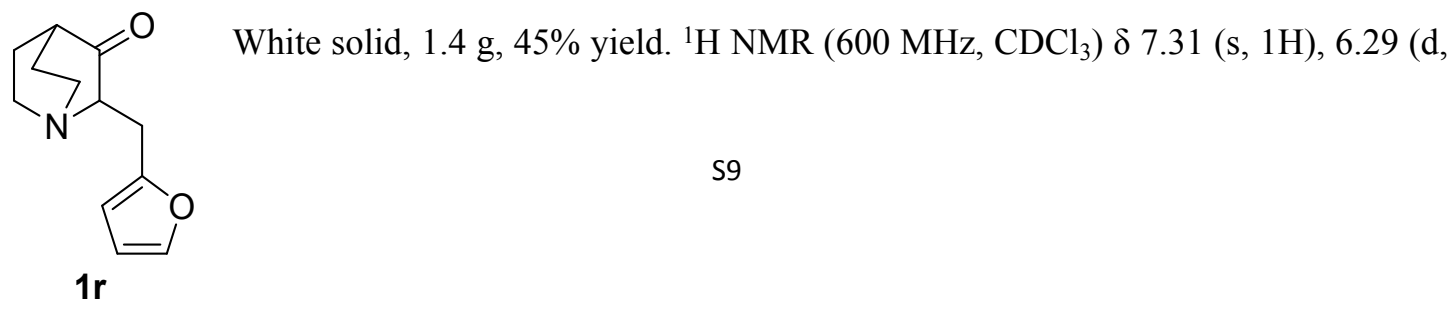


$J=1.9 \mathrm{~Hz}, 1 \mathrm{H}), 6.11(\mathrm{~d}, J=2.8 \mathrm{~Hz}, 1 \mathrm{H}), 3.20(\mathrm{dd}, J=15.8,4.2 \mathrm{~Hz}, 1 \mathrm{H}), 3.10(\mathrm{tt}, J=5.5,5.1 \mathrm{~Hz}$, 2H), 2.95 (ddd, $J=10.1,8.4,2.2 \mathrm{~Hz}, 1 \mathrm{H}), 2.86(\mathrm{dt}, J=10.2,8.2 \mathrm{~Hz}, 2 \mathrm{H}), 2.47-2.41$ (m, 1H), $2.06-1.92(\mathrm{~m}, 4 \mathrm{H}) .{ }^{13} \mathrm{C} \mathrm{NMR}\left(151 \mathrm{MHz}, \mathrm{CDCl}_{3}\right) \delta 220.4,152.6,141.3,110.4,106.5,68.9,48.8$, $41.0,40.0,26.7,26.6,25.0$.

\section{2-((5-Methylthiophen-2-yl)methyl)quinuclidin-3-one (1s):}

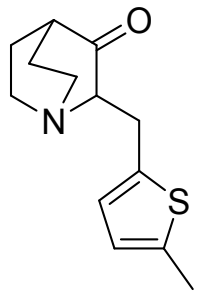

$1 \mathrm{~s}$

White solid, $2.0 \mathrm{~g}, 57 \%$ yield. ${ }^{1} \mathrm{H}$ NMR $\left(400 \mathrm{MHz}, \mathrm{CDCl}_{3}\right) \delta 6.68(\mathrm{~d}, J=3.2 \mathrm{~Hz}$, 1H), $6.57(\mathrm{dd}, J=3.2,1.0 \mathrm{~Hz}, 1 \mathrm{H}), 3.44-3.30(\mathrm{~m}, 2 \mathrm{H}), 3.26-3.10(\mathrm{~m}, 2 \mathrm{H}), 3.04$ $-2.83(\mathrm{~m}, 3 \mathrm{H}), 2.53-2.46(\mathrm{~m}, 1 \mathrm{H}), 2.44(\mathrm{~s}, 3 \mathrm{H}), 2.12-1.89(\mathrm{~m}, 4 \mathrm{H}) .{ }^{13} \mathrm{C}$ NMR $\left(151 \mathrm{MHz}, \mathrm{CDCl}_{3}\right) \delta 220.7,138.9,138.4,125.3,124.6,71.4,48.9,41.0,40.0$, 28.3, 26.9, 25.1, 15.3. HRMS (ESI-TOF) m/z: $[\mathrm{M}+\mathrm{H}]^{+}$Calcd. for $\left(\mathrm{C}_{13} \mathrm{H}_{18} \mathrm{NOS}\right)$ 236.1109; Found 236.1102.

\section{2-Benzhydrylquinuclidin-3-one (1t) ${ }^{12}$ :}<smiles>O=C1C2CCN(CC2)C1C(c1ccccc1)c1ccccc1</smiles>

White solid, $1.9 \mathrm{~g}, 82 \%$ yield. ${ }^{1} \mathrm{H}$ NMR $\left(600 \mathrm{MHz}, \mathrm{CDCl}_{3}\right) \delta 7.42(\mathrm{~d}, J=$ $7.4 \mathrm{~Hz}, 2 \mathrm{H}), 7.34-7.29$ (m, 4H), 7.28 (d, $J=0.9 \mathrm{~Hz}, 1 \mathrm{H}), 7.27$ (s, 1H), 7.20 (dt, $J=19.3,7.3 \mathrm{~Hz}, 2 \mathrm{H}), 4.55(\mathrm{~d}, J=8.0 \mathrm{~Hz}, 1 \mathrm{H}), 4.01(\mathrm{~d}, J=8.0 \mathrm{~Hz}, 1 \mathrm{H})$, $3.18-3.01(\mathrm{~m}, 2 \mathrm{H}), 2.70-2.52(\mathrm{~m}, 2 \mathrm{H}), 2.45(\mathrm{p}, J=2.9 \mathrm{~Hz}, 1 \mathrm{H}), 2.09-$ $1.95(\mathrm{~m}, 2 \mathrm{H}), 1.96-1.84(\mathrm{~m}, 2 \mathrm{H}) .{ }^{13} \mathrm{C}$ NMR $\left(101 \mathrm{MHz}, \mathrm{CDCl}_{3}\right) \delta{ }^{13} \mathrm{C} \mathrm{NMR}$ $\left(151 \mathrm{MHz}, \mathrm{CDCl}_{3}\right) \delta 219.7,143.1,142.2,128.5,128.5,128.4,126.6,126.5,72.5,50.4,49.7,41.8$, $40.9,26.7,25.8$.

\section{2-(Bis(4-fluorophenyl)methyl)quinuclidin-3-one (1u) ${ }^{1}$ :}<smiles>O=C1C2CCN(C2)C1C(c1ccc(F)cc1)c1ccc(F)cc1</smiles>

White solid, $1.1 \mathrm{~g}, 41 \%$ yield. ${ }^{1} \mathrm{H}$ NMR $\left(400 \mathrm{MHz}, \mathrm{CDCl}_{3}\right) \delta 7.42-7.32$ (m, 2H), $7.27-7.18(\mathrm{~m}, 2 \mathrm{H}), 6.98(\mathrm{dt}, J=14.7,8.7 \mathrm{~Hz}, 4 \mathrm{H}), 4.54$ (d, $J=$ $7.8 \mathrm{~Hz}, 1 \mathrm{H}), 3.90$ (d, $J=7.7 \mathrm{~Hz}, 1 \mathrm{H}), 3.20-2.97$ (m, 2H), 2.58 (t, $J=7.9$ $\mathrm{Hz}, 2 \mathrm{H}), 2.51-2.39$ (m, 1H), 1.96 (dddd, $J=16.1,11.3,9.4,2.4 \mathrm{~Hz}, 4 \mathrm{H})$. ${ }^{13} \mathrm{C}$ NMR $\left(151 \mathrm{MHz}, \mathrm{CDCl}_{3}\right) \delta 219.6,162.4,162.3,160.8,160.72,138.8$,

138.7, 137.7, 137.7, 129.9, 129.9, 129.8, 129.8, 115.4, 115.4, 115.3, 115.2, 72.5, 49.7, 48.6, 42.0, $40.8,26.9,25.5$. 


\section{2-(Bis(4-methoxyphenyl)methyl)quinuclidin-3-one (1v) ${ }^{1}$ :}

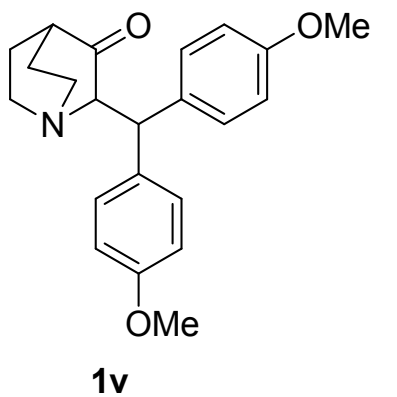

White solid, $1.2 \mathrm{~g}, 44 \%$ yield. ${ }^{1} \mathrm{H}$ NMR $\left(400 \mathrm{MHz}, \mathrm{CDCl}_{3}\right) \delta 7.31$ (d, $J=8.6 \mathrm{~Hz}, 2 \mathrm{H}), 7.19(\mathrm{~d}, J=8.6 \mathrm{~Hz}, 2 \mathrm{H}), 6.90-6.70(\mathrm{~m}, 4 \mathrm{H}), 4.48(\mathrm{~d}$, $J=7.8 \mathrm{~Hz}, 1 \mathrm{H}), 3.91(\mathrm{~d}, J=7.7 \mathrm{~Hz}, 1 \mathrm{H}), 3.76(\mathrm{t}, J=12.2 \mathrm{~Hz}, 6 \mathrm{H})$, $3.18-2.97(\mathrm{~m}, 2 \mathrm{H}), 2.72-2.48(\mathrm{~m}, 2 \mathrm{H}), 2.48-2.36(\mathrm{~m}, 1 \mathrm{H}), 2.09-$ $1.96(\mathrm{~m}, 2 \mathrm{H}), 1.94-1.84(\mathrm{~m}, 2 \mathrm{H}) .{ }^{13} \mathrm{C}$ NMR $\left(101 \mathrm{MHz}, \mathrm{CDCl}_{3}\right) \delta$ $219.9,158.1,158.0,135.6,134.7,129.3,129.3,113.8,113.8,72.9$, $55.2,55.1,49.7,48.7,41.9,40.9,26.6,25.9$.

\section{2-(Di(naphthalen-1-yl)methyl)quinuclidin-3-one (1w)}<smiles>O=C1C2CCN(CC2)C1C(c1cccc2ccccc12)c1cccc2ccccc12</smiles>

$1 w$

White solid, $1.2 \mathrm{~g}, 37 \%$ yield. ${ }^{1} \mathrm{H}$ NMR $\left(400 \mathrm{MHz}, \mathrm{CDCl}_{3}\right) \delta 8.61(\mathrm{~d}, J=$ $8.6 \mathrm{~Hz}, 1 \mathrm{H}), 7.98(\mathrm{dd}, J=7.6,3.4 \mathrm{~Hz}, 2 \mathrm{H}), 7.86(\mathrm{t}, J=9.0 \mathrm{~Hz}, 2 \mathrm{H}), 7.79$ $(\mathrm{d}, J=8.2 \mathrm{~Hz}, 1 \mathrm{H}), 7.72(\mathrm{~d}, J=8.1 \mathrm{~Hz}, 1 \mathrm{H}), 7.61(\mathrm{t}, J=7.3 \mathrm{~Hz}, 1 \mathrm{H}), 7.58$ -7.44 (m, 3H), 7.41 (t, $J=7.1 \mathrm{~Hz}, 1 \mathrm{H}), 7.38-7.30$ (m, 2H), 6.51 (d, $J=$ $3.4 \mathrm{~Hz}, 1 \mathrm{H}), 4.29(\mathrm{~d}, J=3.1 \mathrm{~Hz}, 1 \mathrm{H}), 3.41-3.25(\mathrm{~m}, 1 \mathrm{H}), 3.25-3.09(\mathrm{~m}$, 1H), $2.52-2.43(\mathrm{~m}, 1 \mathrm{H}), 2.42-2.26(\mathrm{~m}, 1 \mathrm{H}), 2.13-1.92(\mathrm{~m}, 2 \mathrm{H}), 1.82(\mathrm{t}, J=12.4 \mathrm{~Hz}, 1 \mathrm{H}), 1.76$

- $1.67(\mathrm{~m}, 1 \mathrm{H}), 1.56-1.40(\mathrm{~m}, 1 \mathrm{H}) .{ }^{13} \mathrm{C} \mathrm{NMR}\left(151 \mathrm{MHz}, \mathrm{CDCl}_{3}\right) \delta 219.8,141.1,137.0,134.0$, $133.9,131.8,131.7,129.0,128.9,127.9,127.4,127.4,126.5,126.3,125.6,125.5,125.4,125.4$, 124.0, 123.5, 73.8, 50.4, 42.9, 40.8, 38.9, 26.8, 25.6. HRMS (ESI-TOF) m/z: $[\mathrm{M}+\mathrm{H}]^{+}$Calcd. for $\left(\mathrm{C}_{28} \mathrm{H}_{26} \mathrm{NO}\right)$ 392.2014; Found 392.2008. 


\section{General procedure for asymmetric transfer hydrogenation of 3-quinuclidinones (1a-w)}

To a round-bottom tube was charged with ammonium formate $(6.0 \mathrm{mmol})$, IPA $(8 \mathrm{~mL})$ and $\mathrm{CH}_{2} \mathrm{Cl}_{2}(8 \mathrm{~mL})$, followed by addition of substrate $\mathbf{1}(1.2 \mathrm{mmol})$ and $(R, R)-\mathbf{C 3}(1.0 \mathrm{~mol} \%)$. The mixture was then stirred at $50{ }^{\circ} \mathrm{C}$ in an oil bath for $72 \mathrm{~h}$. After completion of the reaction, the solvents were removed under vacuum. The crude mixture was finally purified by flash column chromatography $\left(\mathrm{MeOH} / \mathrm{CH}_{2} \mathrm{Cl}_{2}=1 / 40\right.$ to $\left.1 / 10\right)$ to give the desired product with mainly cis configuration. The enantiomeric excesses and diastereomeric ratios were determined by chiral HPLC analysis (Daicel Chiralpak or Phenomenex Chiral column).

(2S,3S)-2-Benzylquinuclidin-3-ol (2a):<smiles>OC1C2CCN(C2)C1Cc1ccccc1</smiles>

$2 a$ White solid (250 mg, 96\% yield); mp 170-171 ${ }^{\circ} \mathrm{C} ;[\alpha]_{\mathrm{D}}^{26}-94.0,(\mathrm{c}=0.4, \mathrm{MeOH}) .{ }^{1} \mathrm{H}$ NMR (400 MHz, $\left.\mathrm{CDCl}_{3}\right) \delta 8.54(\mathrm{~s}, 1 \mathrm{H}), 7.37$ (d, $\left.J=7.3 \mathrm{~Hz}, 2 \mathrm{H}\right), 7.30$ (t, $J=6.1$ $\mathrm{Hz}, 2 \mathrm{H}), 7.23(\mathrm{t}, J=7.2 \mathrm{~Hz}, 1 \mathrm{H}), 4.05-3.88(\mathrm{~m}, 1 \mathrm{H}), 3.59(\mathrm{t}, J=7.9 \mathrm{~Hz}, 1 \mathrm{H})$, $3.36(\mathrm{dd}, J=25.3,13.4 \mathrm{~Hz}, 3 \mathrm{H}), 3.21(\mathrm{~d}, J=12.5 \mathrm{~Hz}, 1 \mathrm{H}), 3.15-2.99(\mathrm{~m}, 2 \mathrm{H})$, $2.25(\mathrm{dd}, J=20.3,9.7 \mathrm{~Hz}, 1 \mathrm{H}), 1.86(\mathrm{dd}, J=17.1,8.4 \mathrm{~Hz}, 1 \mathrm{H}), 1.76-1.51(\mathrm{~m}, 2 \mathrm{H}), 1.28(\mathrm{~d}, J=$ $12.0 \mathrm{~Hz}, 1 \mathrm{H}) .{ }^{13} \mathrm{C} \mathrm{NMR}\left(151 \mathrm{MHz}, \mathrm{CDCl}_{3}\right) \delta 169.1,137.3,129.2,128.8,126.8,65.2,62.5,46.9$, 41.1, 30.8, 28.0, 21.1, 17.2. Enantiometric excess: >99\%, determined by HPLC (Daicel Chiralpak IE column, hexane/EtOH/DEA 80:20:0.1, flow rate $0.5 \mathrm{~mL} / \mathrm{min}, \mathrm{t}_{\text {major }}=22.21 \mathrm{~min}, \lambda=210 \mathrm{~nm}$ ). HRMS (ESI-TOF) m/z: [M+H] $]^{+}$Calcd. for $\mathrm{C}_{14} \mathrm{H}_{20} \mathrm{NO} 218.1545$; Found 218.1545.

\section{(2S,3S)-2-(4-Methoxybenzyl)quinuclidin-3-ol (2b):}

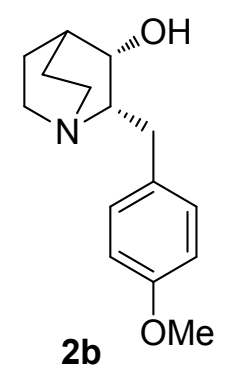

White solid (291 mg, 98\% yield); mp 141-142 ${ }^{\circ} \mathrm{C} ;[\alpha]_{\mathrm{D}}^{26}-78.5,(\mathrm{c}=0.4, \mathrm{MeOH})$. ${ }^{1} \mathrm{H}$ NMR $\left(400 \mathrm{MHz}, \mathrm{CDCl}_{3}\right) \delta 7.24(\mathrm{~d}, J=8.5 \mathrm{~Hz}, 2 \mathrm{H}), 6.86(\mathrm{~d}, J=8.6 \mathrm{~Hz}, 2 \mathrm{H})$, $3.89(\mathrm{t}, J=5.0 \mathrm{~Hz}, 1 \mathrm{H}), 3.80(\mathrm{~s}, 3 \mathrm{H}), 3.27-3.09(\mathrm{~m}, 3 \mathrm{H}), 2.84(\mathrm{dd}, J=17.9,9.0$ $\mathrm{Hz}, 3 \mathrm{H}), 2.72(\mathrm{t}, J=12.2 \mathrm{~Hz}, 1 \mathrm{H}), 2.07-1.86(\mathrm{~m}, 3 \mathrm{H}), 1.65(\mathrm{dt}, J=8.5,6.5 \mathrm{~Hz}$, $157.9,132.3,129.7,114.1,69.0,62.0,55.2,48.9,41.79,32.9,28.8,24.8,19.0$. Enantiometric excess: $>99 \%$, determined by HPLC (Daicel Chiralpak IE column, hexane/EtOH/DEA 60:40:0.1, flow rate $0.5 \mathrm{~mL} / \mathrm{min}, \mathrm{t}_{\text {major }}=16.8 \mathrm{~min}, \lambda=210 \mathrm{~nm}$ ). HRMS (ESI-TOF) $\mathrm{m} / \mathrm{z}:[\mathrm{M}+\mathrm{H}]^{+}$Calcd. for $\mathrm{C}_{15} \mathrm{H}_{22} \mathrm{NO}_{2}$ 248.1651; Found 248.1648.

(2S,3S)-2-(3-Methylbenzyl)quinuclidin-3-ol (2c): 


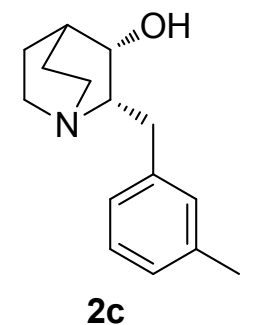

White solid (269 mg, 97\% yield); $\mathrm{mp} 142-143{ }^{\circ} \mathrm{C} ;[\alpha]_{\mathrm{D}}^{26}-88.5,(\mathrm{c}=0.4, \mathrm{MeOH})$. ${ }^{1} \mathrm{H} \mathrm{NMR}\left(600 \mathrm{MHz}, \mathrm{CDCl}_{3}\right) \delta 7.21(\mathrm{t}, J=7.5 \mathrm{~Hz}, 1 \mathrm{H}), 7.13(\mathrm{~d}, J=9.2 \mathrm{~Hz}, 2 \mathrm{H})$, $7.03(\mathrm{~d}, J=7.4 \mathrm{~Hz}, 1 \mathrm{H}), 3.90(\mathrm{~s}, 1 \mathrm{H}), 3.26-3.12(\mathrm{~m}, 3 \mathrm{H}), 2.85(\mathrm{dt}, J=13.9,9.0$

$\mathrm{Hz}, 3 \mathrm{H}), 2.78-2.67(\mathrm{~m}, 1 \mathrm{H}), 2.35$ (s, 3H), $2.00-1.87(\mathrm{~m}, 2 \mathrm{H}), 1.81(\mathrm{~s}, 1 \mathrm{H})$, $1.67(\mathrm{ddd}, \mathrm{J}=12.9,10.2,5.7 \mathrm{~Hz}, 1 \mathrm{H}), 1.58-1.43(\mathrm{~m}, 1 \mathrm{H}), 1.40-1.25(\mathrm{~m}, 1 \mathrm{H})$. ${ }^{13} \mathrm{C}$ NMR (151 MHz, CDCl3) $\delta 140.2,138.2,129.6,128.6,126.9,125.8,68.9,61.8,48.9,41.8$, 33.8, 28.7, 24.7, 21.5, 19.0. Enantiometric excess: >99\%, determined by HPLC (Daicel Chiralpak IE column, hexane/EtOH/DEA 80:20:0.1, flow rate $0.5 \mathrm{~mL} / \mathrm{min}, \mathrm{t}_{\text {major }}=24.65 \mathrm{~min}, \lambda=210 \mathrm{~nm}$ ). HRMS (ESI-TOF) m/z: [M+H] $]^{+}$Calcd. for $\mathrm{C}_{15} \mathrm{H}_{22} \mathrm{NO} 232.1701$; Found 232.1703 .

\section{(2S,3S)-2-(3,4-Dimethylbenzyl)quinuclidin-3-ol (2d):}

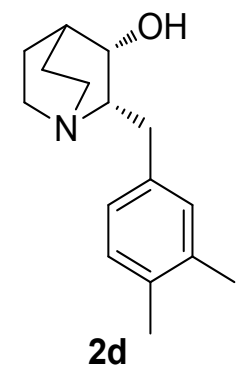

White solid (289 mg, 98\% yield); mp $154-155^{\circ} \mathrm{C} ;[\alpha]_{\mathrm{D}}^{26}-73.0,(\mathrm{c}=0.4, \mathrm{MeOH})$. ${ }^{1} \mathrm{H}$ NMR (400 MHz, DMSO- $\left.d_{6}\right) \delta 7.12(\mathrm{~s}, 1 \mathrm{H}), 7.07$ (s, 2H), 5.39 (d, $J=4.1 \mathrm{~Hz}$, $1 \mathrm{H}), 3.91-3.83(\mathrm{~m}, 1 \mathrm{H}), 3.71(\mathrm{dd}, J=12.0,8.2 \mathrm{~Hz}, 1 \mathrm{H}), 3.22(\mathrm{dt}, J=17.9,9.1$ Hz, 3H), $3.13-2.89$ (m, 3H), 2.19 (d, J=6.0 Hz, 6H), 2.04 (s, 2H), $1.86-1.66$ $(\mathrm{m}, 2 \mathrm{H}), 1.59(\mathrm{dd}, J=19.1,10.6 \mathrm{~Hz}, 1 \mathrm{H}) .{ }^{13} \mathrm{C}$ NMR $\left(101 \mathrm{MHz}, \mathrm{DMSO}-d_{6}\right) \delta$ 136.3, 135.1, 134.5, 130.8, 129.9, 127.0, 64.3, 62.7, 47.4, 41.5, 30.2, 28.0, 20.4, 19.9, 19.4, 17.0. Enantiometric excess: $>99 \%$, determined by HPLC (Daicel Chiralpak IE column, hexane/EtOH/DEA 80:20:0.1, flow rate $0.5 \mathrm{~mL} / \mathrm{min}, \mathrm{t}_{\text {major }}=24.41 \mathrm{~min}, \mathrm{t}_{\text {minor }}=14.12 \mathrm{~min}, \lambda=210$ nm). HRMS (ESI-TOF) m/z: [M+H] $]^{+}$Calcd. for $\mathrm{C}_{16} \mathrm{H}_{24} \mathrm{NO} 246.1858$; Found 246.1853.

\section{N-(3-(((2S,3S)-3-hydroxyquinuclidin-2-yl)methyl)phenyl)acetamide (2e):}

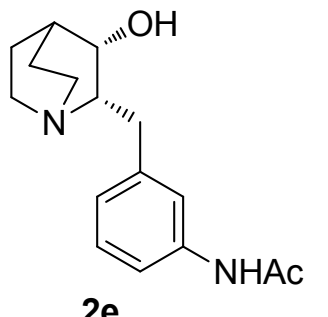

White solid (316 mg, 96\% yield); mp $133-134{ }^{\circ} \mathrm{C} ;[\alpha]_{\mathrm{D}}^{26} \quad-46.5$, $(\mathrm{c}=0.4$, MeOH). ${ }^{1} \mathrm{H}$ NMR (600 MHz, DMSO-d 6 ) $\delta 9.91(\mathrm{~s}, 1 \mathrm{H}), 8.36(\mathrm{~s}, 1 \mathrm{H}), 7.44$ $(\mathrm{d}, J=11.8 \mathrm{~Hz}, 2 \mathrm{H}), 7.17(\mathrm{t}, J=7.7 \mathrm{~Hz}, 1 \mathrm{H}), 6.98(\mathrm{~d}, J=7.5 \mathrm{~Hz}, 1 \mathrm{H}), 3.88$ $-3.75(\mathrm{~m}, 1 \mathrm{H}), 3.34(\mathrm{~d}, J=6.6 \mathrm{~Hz}, 1 \mathrm{H}), 3.12(\mathrm{dt}, J=13.2,9.9 \mathrm{~Hz}, 2 \mathrm{H})$, $2.89(\mathrm{dd}, J=19.0,10.7 \mathrm{~Hz}, 2 \mathrm{H}), 2.80(\mathrm{dd}, J=13.5,6.6 \mathrm{~Hz}, 2 \mathrm{H}), 2.03(\mathrm{~s}$, 3H), 1.96 (t, $J=11.0 \mathrm{~Hz}, 1 \mathrm{H}), 1.92(\mathrm{~s}, 1 \mathrm{H}), 1.74-1.63(\mathrm{~m}, 1 \mathrm{H}), 1.59$ (dd, $J=20.3,9.9 \mathrm{~Hz}, 1 \mathrm{H})$, $1.40(\mathrm{dd}, J=19.1,10.6 \mathrm{~Hz}, 1 \mathrm{H}) .{ }^{13} \mathrm{C}$ NMR $\left(151 \mathrm{MHz}, \mathrm{DMSO}-d_{6}\right) \delta 168.6,165.7,140.4,139.6$, $128.7,124.3,120.4,117.3,66.0,62.3,48.0,31.7,28.7,24.4,22.4,18.1$. Enantiometric 
excess: $>99 \%$, determined by HPLC (Phenomenex Chiral MX column, hexane/EtOH/DEA 82:18:0.1, flow rate $0.5 \mathrm{~mL} / \mathrm{min}, \mathrm{t}_{\text {major }}=21.82 \mathrm{~min}, \lambda=210 \mathrm{~nm}$ ). HRMS (ESI-TOF) $\mathrm{m} / \mathrm{z}:[\mathrm{M}+\mathrm{H}]^{+}$ Calcd. for $\mathrm{C}_{16} \mathrm{H}_{23} \mathrm{~N}_{2} \mathrm{O}_{2}$ 275.1760; Found 275.1762.

\section{(2S,3S)-2-(2-Hydroxybenzyl)quinuclidin-3-ol (2f):}<smiles>Oc1ccccc1C[C@@H]1CC2CCN1CC2</smiles>

$2 f$

White solid (255 mg, 91\% yield); mp $139-140{ }^{\circ} \mathrm{C} ;[\alpha]_{\mathrm{D}}^{26}-161.0,(\mathrm{c}=0.4$, MeOH). ${ }^{1} \mathrm{H}$ NMR $\left(600 \mathrm{MHz}, \mathrm{CDCl}_{3}\right) \delta 7.18-7.08(\mathrm{~m}, 1 \mathrm{H}), 7.07-6.99(\mathrm{~m}$, 1H), $6.89-6.81(\mathrm{~m}, 1 \mathrm{H}), 6.74(\mathrm{td}, J=7.4,0.9 \mathrm{~Hz}, 1 \mathrm{H}), 4.08(\mathrm{dd}, J=7.0,5.0$ $\mathrm{Hz}, 1 \mathrm{H}), 3.45$ (ddd, $J=17.7,9.8,1.6 \mathrm{~Hz}, 1 \mathrm{H}), 3.31(\mathrm{dd}, J=15.5,11.0 \mathrm{~Hz}$, 1H), $3.15-3.03(\mathrm{~m}, 1 \mathrm{H}), 2.97-2.78(\mathrm{~m}, 3 \mathrm{H}), 2.69(\mathrm{~d}, J=15.5 \mathrm{~Hz}, 1 \mathrm{H}), 2.13$ $-1.98(\mathrm{~m}, 2 \mathrm{H}), 1.75(\mathrm{ddd}, J=13.2,8.2,4.2 \mathrm{~Hz}, 1 \mathrm{H}), 1.60-1.49(\mathrm{~m}, 1 \mathrm{H}), 1.50-1.39(\mathrm{~m}, 1 \mathrm{H})$. ${ }^{13} \mathrm{C}$ NMR $\left(151 \mathrm{MHz}, \mathrm{CDCl}_{3}\right) \delta 158.1,131.0,128.2,126.8,118.6,117.9,69.0,62.6,47.2,40.7$, 30.5, 28.6, 23.4, 18.5. Enantiometric excess: $>99 \%$, determined by HPLC (Daicel Chiralpak IE column, hexane/EtOH/DEA 80:20:0.1, flow rate $0.5 \mathrm{~mL} / \mathrm{min}$, $\mathrm{t}_{\text {major }}=24.69 \mathrm{~min}, \lambda=210 \mathrm{~nm}$ ). HRMS (ESI-TOF) m/z: [M+H] $]^{+}$Calcd. for $\mathrm{C}_{14} \mathrm{H}_{20} \mathrm{NO}_{2}$ 234.1494; Found 234.1492.

\section{(2S,3S)-2-(2-Fluorobenzyl)quinuclidin-3-ol (2g):}<smiles>O[C@@H]1C2CCN(C2)[C@H]1Cc1ccccc1F</smiles>

$2 \mathrm{~g}$
White solid (271 mg, 96\% yield); mp 176-177 ${ }^{\circ} \mathrm{C} ;[\alpha]_{\mathrm{D}}^{26} \quad-71.0,(\mathrm{c}=0.4$, MeOH). ${ }^{1} \mathrm{H}$ NMR (400 MHz, $\left.\mathrm{CDCl}_{3}\right) \delta 7.37-7.29(\mathrm{~m}, 1 \mathrm{H}), 7.19$ (td, $J=7.4$, $1.6 \mathrm{~Hz}, 1 \mathrm{H}), 7.06(\mathrm{ddd}, J=23.1,13.0,7.6 \mathrm{~Hz}, 2 \mathrm{H}), 4.09-3.80(\mathrm{~m}, 1 \mathrm{H}), 3.27-$ $3.10(\mathrm{~m}, 3 \mathrm{H}), 2.98-2.65(\mathrm{~m}, 4 \mathrm{H}), 1.97(\mathrm{dd}, J=10.4,6.5 \mathrm{~Hz}, 3 \mathrm{H}), 1.66(\mathrm{qd}, J$ $=8.9,3.9 \mathrm{~Hz}, 1 \mathrm{H}), 1.50(\mathrm{ddd}, J=12.9,9.0,5.0 \mathrm{~Hz}, 1 \mathrm{H}), 1.40-1.28(\mathrm{~m}, 1 \mathrm{H})$. ${ }^{13} \mathrm{C}$ NMR $\left(151 \mathrm{MHz}, \mathrm{CDCl}_{3}\right) \delta 162.2,160.5,131.2,131.2,127.7,127.6,127.5,127.4$ 124.1, 124.1, 115.4, 115.2, 69.1, 60.9, 48.8, 41.5, 29.0, 26.8, 24.7, 18.9. Enantiometric excess: 97\%, determined by HPLC (Daicel Chiralpak IE column, hexane/EtOH/DEA 80:20:0.1, flow rate 0.5 $\left.\mathrm{mL} / \mathrm{min}, \mathrm{t}_{\text {major }}=15.98 \mathrm{~min}, \mathrm{t}_{\text {minor }}=11.80 \mathrm{~min}, \lambda=210 \mathrm{~nm}\right)$. HRMS $\left(\right.$ ESI-TOF) $\mathrm{m} / \mathrm{z}:[\mathrm{M}+\mathrm{H}]^{+}$ Calcd. for $\mathrm{C}_{14} \mathrm{H}_{19} \mathrm{FNO} 236.1451$; Found 236.1449.

(2S,3S)-2-(3-Fluorobenzyl)quinuclidin-3-ol (2h): 


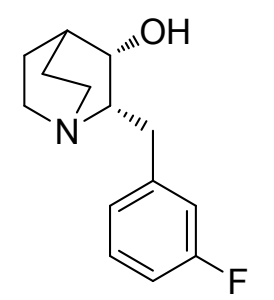

$2 \mathrm{~h}$

White solid (274 mg, 97\% yield); mp 171-172 ${ }^{\circ} \mathrm{C} ;[\alpha]_{\mathrm{D}}^{26} \quad-83.9,(\mathrm{c}=0.4$, MeOH). ${ }^{1} \mathrm{H}$ NMR $\left(600 \mathrm{MHz}, \mathrm{CDCl}_{3}\right) \delta 7.27(\mathrm{dd}, J=12.9,5.1 \mathrm{~Hz}, 1 \mathrm{H}), 7.09$ (d, $J=7.6 \mathrm{~Hz}, 1 \mathrm{H}), 7.04(\mathrm{~d}, J=10.1 \mathrm{~Hz}, 1 \mathrm{H}), 6.90(\mathrm{td}, J=8.4,1.9 \mathrm{~Hz}, 1 \mathrm{H}), 3.91$

$(\mathrm{dd}, J=6.9,4.2 \mathrm{~Hz}, 1 \mathrm{H}), 3.29-3.08(\mathrm{~m}, 3 \mathrm{H}), 2.84(\mathrm{tdd}, J=15.9,11.3,4.2 \mathrm{~Hz}$, $3 \mathrm{H}), 2.72(\mathrm{t}, J=11.4 \mathrm{~Hz}, 1 \mathrm{H}), 1.95(\mathrm{dd}, J=16.4,13.2 \mathrm{~Hz}, 3 \mathrm{H}), 1.67(\mathrm{qd}, J=$ 8.6, $3.8 \mathrm{~Hz}, 1 \mathrm{H}), 1.56-1.41(\mathrm{~m}, 1 \mathrm{H}), 1.40-1.22(\mathrm{~m}, 1 \mathrm{H}) .{ }^{13} \mathrm{C} \mathrm{NMR}\left(151 \mathrm{MHz}, \mathrm{CDCl}_{3}\right) \delta 163.8$, $162.1,143.3,143.3,129.9,129.8,124.6,124.5,115.9,115.7,113.0,112.8,68.8,61.8,48.8,41.70$, 33.5, 33.5, 29.0, 24.6, 18.9. Enantiometric excess: 98\%, determined by HPLC (Daicel Chiralpak IE column, hexane/EtOH/DEA 80:20:0.1, flow rate $0.5 \mathrm{~mL} / \mathrm{min}, \mathrm{t}_{\text {major }}=15.74 \mathrm{~min}, \mathrm{t}_{\text {minor }}=11.58$ min, $\lambda=210 \mathrm{~nm}$ ). HRMS (ESI-TOF) m/z: $[\mathrm{M}+\mathrm{H}]^{+}$Calcd. for $\mathrm{C}_{14} \mathrm{H}_{19} \mathrm{FNO} 236.1451$; Found 236.1448 .

(2S,3S)-2-([1,1'-Biphenyl]-4-ylmethyl)quinuclidin-3-ol (2i):<smiles>OC1C2CCN(C2)C1Cc1ccc(-c2ccccc2)cc1</smiles>

2i

White solid (348.5 mg, 99\% yield); mp 190-191 ${ }^{\circ} \mathrm{C} ;[\alpha]_{\mathrm{D}}^{26}-71.0,(\mathrm{c}=0.4, \mathrm{MeOH})$. ${ }^{1} \mathrm{H}$ NMR (400 MHz, DMSO- $\left.d_{6}\right) \delta 7.62(\mathrm{~d}, J=7.4 \mathrm{~Hz}, 2 \mathrm{H}), 7.51(\mathrm{~d}, J=8.1 \mathrm{~Hz}$, 2H), $7.45(\mathrm{t}, J=7.6 \mathrm{~Hz}, 2 \mathrm{H}), 7.32(\mathrm{dd}, J=14.8,7.7 \mathrm{~Hz}, 3 \mathrm{H}), 4.67(\mathrm{~d}, J=3.7 \mathrm{~Hz}$, 1H), $3.87-3.70(\mathrm{~m}, 1 \mathrm{H}), 3.11-2.88(\mathrm{~m}, 3 \mathrm{H}), 2.78(\mathrm{dd}, J=14.3,9.7 \mathrm{~Hz}, 1 \mathrm{H}), 2.68$ - $2.50(\mathrm{~m}, 3 \mathrm{H}), 1.85$ (t, $J=9.8 \mathrm{~Hz}, 1 \mathrm{H}), 1.76(\mathrm{~s}, 1 \mathrm{H}), 1.59-1.47$ (m, 1H), 1.47 $1.34(\mathrm{~m}, 1 \mathrm{H}), 1.17(\mathrm{dd}, J=18.3,11.1 \mathrm{~Hz}, 1 \mathrm{H}) \cdot{ }^{13} \mathrm{C}$ NMR $\left(151 \mathrm{MHz}\right.$, DMSO- $\left.d_{6}\right) \delta$ $141.7,140.8,137.6,130.1,129.3,127.4,126.9,126.6,7.14,62.4,49.0,41.27,32.68,29.41,24.99$, 19.54. Enantiometric excess: 99\%, determined by HPLC (Daicel Chiralpak IE column, hexane/EtOH/DEA 60:40:0.1, flow rate $0.5 \mathrm{~mL} / \mathrm{min}, \mathrm{t}_{\text {major }}=16.55 \mathrm{~min}, \mathrm{t}_{\text {minor }}=11.26 \mathrm{~min}, \lambda=210$ nm). HRMS (ESI-TOF) m/z: [M+H] $]^{+}$Calcd. for $\mathrm{C}_{20} \mathrm{H}_{24} \mathrm{NO}$ 294.1858; Found 294.1856.

(2S,3S)-2-(4-Fluorobenzyl)quinuclidin-3-ol (2j):<smiles>O[C@@H]1C2CCN(C2)[C@H]1Cc1ccc(F)cc1</smiles>

White solid (274 mg, 97\% yield); mp 160-161 ${ }^{\circ} \mathrm{C} ;[\alpha]_{\mathrm{D}}^{26}-83.5,(\mathrm{c}=0.4, \mathrm{MeOH}) .{ }^{1} \mathrm{H}$ NMR (400 MHz, $\left.\mathrm{CDCl}_{3}\right) \delta 7.33-7.26(\mathrm{~m}, 2 \mathrm{H}), 6.99(\mathrm{t}, J=8.7 \mathrm{~Hz}, 2 \mathrm{H}), 3.89(\mathrm{~s}$, $1 \mathrm{H}), 3.26-3.05(\mathrm{~m}, 3 \mathrm{H}), 2.92-2.77(\mathrm{~m}, 3 \mathrm{H}), 2.76-2.65(\mathrm{~m}, 1 \mathrm{H}), 1.91(\mathrm{~d}, J=9.3$ $\mathrm{Hz}, 2 \mathrm{H}), 1.80-1.60(\mathrm{~m}, 2 \mathrm{H}), 1.49(\mathrm{dt}, J=12.9,7.8 \mathrm{~Hz}, 1 \mathrm{H}), 1.33(\mathrm{td}, J=10.8,7.4$ 
$\mathrm{Hz}, 1 \mathrm{H}) .{ }^{13} \mathrm{C} \mathrm{NMR}\left(151 \mathrm{MHz}, \mathrm{CDCl}_{3}\right) \delta 162.1,160.5,136.2,136.1,130.3,130.2,115.3,115.2$, $68.9,62.0,48.9,41.7,32.9,29.0,24.7,18.9$. Enantiometric excess: $>99 \%$, determined by HPLC (Daicel Chiralpak IE column, hexane/EtOH/DEA 80:20:0.1, flow rate $0.5 \mathrm{~mL} / \mathrm{min}$, $\mathrm{t}_{\text {major }}=13.21$ min, $\lambda=210 \mathrm{~nm}$ ). HRMS (ESI-TOF) $\mathrm{m} / \mathrm{z}$ : $[\mathrm{M}+\mathrm{H}]^{+}$Calcd. for $\mathrm{C}_{14} \mathrm{H}_{19} \mathrm{FNO} 236.1451$; Found 236.1453

(2S,3S)-2-(4-(Trifluoromethyl)benzyl)quinuclidin-3-ol (2k):

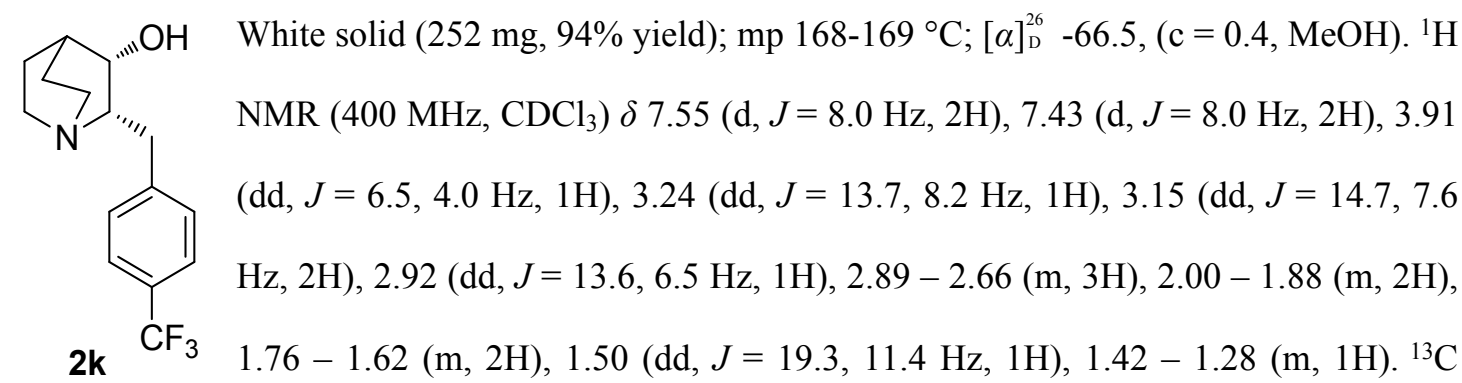
$\operatorname{NMR}\left(151 \mathrm{MHz}, \mathrm{CDCl}_{3}\right) \delta 145.0,129.3,128.5,128.3,128.1,127.9,125.3,125.3,125.3,125.2$, 123.5, 68.9, 61.9, 48.8, 41.6, 33.5, 29.2, 24.6, 18.9. Enantiometric excess: 98\%, determined by HPLC (Phenomenex Chiral MZ (2) column, hexane/EtOH/DEA 96:4:0.1, flow rate $0.5 \mathrm{~mL} / \mathrm{min}$, $\mathrm{t}_{\text {major }}=17.85 \mathrm{~min}, \mathrm{t}_{\text {minor }}=20.64 \mathrm{~min}, \lambda=210 \mathrm{~nm}$ ). HRMS $\left(\right.$ ESI-TOF) $\mathrm{m} / \mathrm{z}:[\mathrm{M}+\mathrm{H}]^{+}$Calcd. for $\mathrm{C}_{15} \mathrm{H}_{19} \mathrm{~F}_{3} \mathrm{NO} 286.1419$; Found 286.1416.

\section{(2S,3S)-2-(2,5-Difluorobenzyl)quinuclidin-3-ol (2I):}

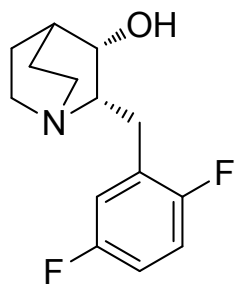

2I

White solid (280 mg, 92\% yield); mp $167-168^{\circ} \mathrm{C} ;[\alpha]_{\mathrm{D}}^{26}-65.5$, $(\mathrm{c}=0.4, \mathrm{MeOH})$. ${ }^{1} \mathrm{H}$ NMR $\left(400 \mathrm{MHz}, \mathrm{CDCl}_{3}\right) \delta 7.04(\mathrm{ddd}, J=8.9,5.7,3.1 \mathrm{~Hz}, 1 \mathrm{H}), 6.97(\operatorname{td}, J=$ 9.1, 4.6 Hz, 1H), $6.85(\mathrm{ddd}, J=11.8,8.1,3.4 \mathrm{~Hz}, 1 \mathrm{H}), 4.00-3.87(\mathrm{~m}, 1 \mathrm{H})$, $3.24-3.06(\mathrm{~m}, 3 \mathrm{H}), 2.94-2.67(\mathrm{~m}, 4 \mathrm{H}), 2.03-1.85(\mathrm{~m}, 3 \mathrm{H}), 1.74-1.60(\mathrm{~m}$, 1H), $1.57-1.43(\mathrm{~m}, 1 \mathrm{H}), 1.34(\mathrm{dt}, J=10.8,7.6 \mathrm{~Hz}, 1 \mathrm{H}) .{ }^{13} \mathrm{C} \mathrm{NMR}(101 \mathrm{MHz}$, $\left.\mathrm{CDCl}_{3}\right) \delta 159.8,159.7,158.5,158.5,157.4,157.4,156.1,156.1,129.6,129.5,129.4,129.3,117.7$, 117.6, 117.4, 117.4, 116.1, 116.0, 115.9, 115.8, 113.9, 113.8, 113.7, 113.6, 69.0, 60.7, 48.8, 41.4, 29.2, 26.6, 24.7, 18.9. Enantiometric excess: 95\%, determined by HPLC (Daicel Chiralpak IE column, hexane/EtOH/DEA 80:20:0.1, flow rate $0.5 \mathrm{~mL} / \mathrm{min}, \mathrm{t}_{\text {major }}=12.82 \mathrm{~min}, \mathrm{t}_{\mathrm{minor}}=10.29 \mathrm{~min}$, $\lambda=210 \mathrm{~nm}$ ). HRMS (ESI-TOF) m/z: [M+H] $]^{+}$Calcd. for $\mathrm{C}_{14} \mathrm{H}_{18} \mathrm{~F}_{2} \mathrm{NO} 254.1356$; Found 254.1356. 


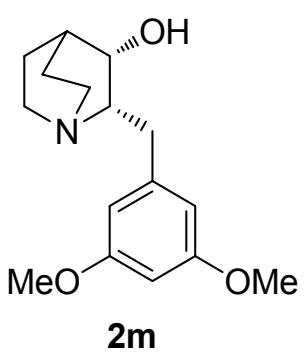

White solid (326 mg, 98\% yield); mp 141-142 ${ }^{\circ} \mathrm{C} ;[\alpha]_{\mathrm{D}}^{26}-72.5$, $(\mathrm{c}=0.4$, MeOH). ${ }^{1} \mathrm{H}$ NMR (400 MHz, $\left.\mathrm{CDCl}_{3}\right) \delta 6.50(\mathrm{~s}, 2 \mathrm{H}), 6.33(\mathrm{~s}, 1 \mathrm{H}), 3.91(\mathrm{~s}$, 1H), 3.79 (s, 6H), $3.25-3.09$ (m, 3H), $2.91-2.77$ (m, 3H), 2.73 (t, $J=$ $11.2 \mathrm{~Hz}, 1 \mathrm{H}), 1.90(\mathrm{~d}, J=22.7 \mathrm{~Hz}, 3 \mathrm{H}), 1.66(\mathrm{dd}, J=11.7,5.5 \mathrm{~Hz}, 1 \mathrm{H})$, $1.50(\mathrm{dd}, J=20.6,9.8 \mathrm{~Hz}, 1 \mathrm{H}), 1.31(\mathrm{dd}, J=18.0,10.7 \mathrm{~Hz}, 1 \mathrm{H}) .{ }^{13} \mathrm{C} \mathrm{NMR}$ $\left(101 \mathrm{MHz}, \mathrm{CDCl}_{3}\right) \delta 161.0,142.7,106.9,98.0,68.8,61.6,55.3,48.9,41.9,34.2,28.7,24.7,18.9$. Enantiometric excess: $>99 \%$, determined by HPLC (Daicel Chiralpak IA column, hexane/EtOH/DEA 60:40:0.1, flow rate $0.5 \mathrm{~mL} / \mathrm{min}, \mathrm{t}_{\text {major }}=21.25 \mathrm{~min}, \mathrm{t}_{\text {minor }}=10.40 \mathrm{~min}, \lambda=210$ nm). HRMS (ESI-TOF) m/z: $[\mathrm{M}+\mathrm{H}]^{+}$Calcd. for $\mathrm{C}_{16} \mathrm{H}_{24} \mathrm{NO}_{3}$ 278.1756; Found 278.1758.

\section{(2S,3S)-2-(3-Hydroxy-4-methoxybenzyl)quinuclidin-3-ol (2n)}

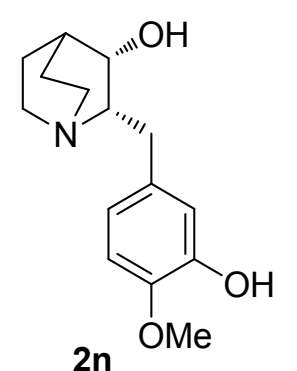

White solid (294 mg, 93\% yield); $[\alpha]_{\mathrm{D}}^{26}-49.0,(\mathrm{c}=0.4, \mathrm{MeOH}) .{ }^{1} \mathrm{H}$ NMR $(400$ $\left.\mathrm{MHz}, \mathrm{CDCl}_{3}\right) \delta 6.86(\mathrm{~s}, 1 \mathrm{H}), 6.79(\mathrm{~d}, J=8.2 \mathrm{~Hz}, 2 \mathrm{H}), 3.95-3.90(\mathrm{~m}, 1 \mathrm{H})$, $3.87(\mathrm{~s}, 3 \mathrm{H}), 3.19(\mathrm{~d}, J=11.2 \mathrm{~Hz}, 3 \mathrm{H}), 2.91(\mathrm{~d}, J=7.9 \mathrm{~Hz}, 2 \mathrm{H}), 2.80(\mathrm{dd}, J=$ 24.8, 10.6 Hz, 3H), $1.97(\mathrm{~s}, 2 \mathrm{H}), 1.75-1.67(\mathrm{~m}, 1 \mathrm{H}), 1.59-1.50(\mathrm{~m}, 1 \mathrm{H})$, $1.33(\mathrm{dt}, J=23.2,13.5 \mathrm{~Hz}, 2 \mathrm{H}) .{ }^{13} \mathrm{C} \mathrm{NMR}\left(101 \mathrm{MHz}, \mathrm{CDCl}_{3}\right) \delta 146.4,145.6$, 132.9, 119.7, 114.9, 111.2, 68.6, 62.0, 55.9, 48.7, 41.70, 33.0, 28.5, 24.4, 18.7. Enantiometric excess: $>99 \%$, determined by HPLC (Daicel Chiralpak IC column, hexane/EtOH/DEA 50:50:0.1, flow rate $0.5 \mathrm{~mL} / \mathrm{min}, \mathrm{t}_{\text {major }}=12.60 \mathrm{~min}, \lambda=210 \mathrm{~nm}$ ). HRMS (ESI-TOF) $\mathrm{m} / \mathrm{z}:[\mathrm{M}+\mathrm{H}]^{+}$Calcd. for $\mathrm{C}_{15} \mathrm{H}_{22} \mathrm{NO}_{3}$ 264.1600; Found 264.1600.

\section{(2S,3S)-2-(5-(Tert-butyl)-2-methoxybenzyl)quinuclidin-3-ol (2o):}

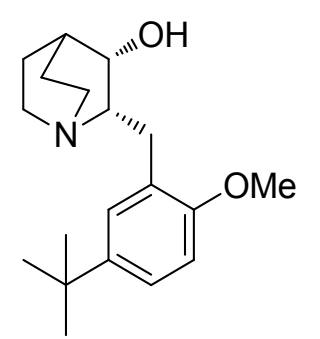

20

White solid (356 mg, 98\% yield). mp $155-156{ }^{\circ} \mathrm{C} ;[\alpha]_{\mathrm{D}}^{26}-37.0,(\mathrm{c}=0.4$, MeOH). ${ }^{1} \mathrm{H}$ NMR $\left(400 \mathrm{MHz}, \mathrm{CDCl}_{3}\right) \delta 7.35(\mathrm{~d}, J=2.3 \mathrm{~Hz}, 1 \mathrm{H}), 7.24(\mathrm{dd}, J$ = 8.6, $2.4 \mathrm{~Hz}, 1 \mathrm{H}), 6.84(\mathrm{~d}, J=8.6 \mathrm{~Hz}, 1 \mathrm{H}), 3.89(\mathrm{~s}, 3 \mathrm{H}), 3.84-3.77(\mathrm{~m}$, 1H), $3.57-3.42(\mathrm{~m}, 2 \mathrm{H}), 3.25-3.09(\mathrm{~m}, 1 \mathrm{H}), 3.02-2.88(\mathrm{~m}, 2 \mathrm{H}), 2.84$ $(\mathrm{dd}, J=13.2,8.8 \mathrm{~Hz}, 1 \mathrm{H}), 2.76(\mathrm{t}, J=11.8 \mathrm{~Hz}, 1 \mathrm{H}), 2.67(\mathrm{dd}, J=13.6,3.0$ $\mathrm{Hz}, 1 \mathrm{H}), 2.07$ (d, $J=6.5 \mathrm{~Hz}, 1 \mathrm{H}), 2.04-1.96(\mathrm{~m}, 2 \mathrm{H}), 1.64(\mathrm{tt}, J=9.1,3.6$ $\mathrm{Hz}, 1 \mathrm{H}), 1.46$ (ddd, $J=12.6,10.4,8.2 \mathrm{~Hz}, 1 \mathrm{H}), 1.31(\mathrm{~s}, 9 \mathrm{H}) .{ }^{13} \mathrm{C} \mathrm{NMR}\left(101 \mathrm{MHz}, \mathrm{CDCl}_{3}\right) \delta$ 
$154.2,144.3,128.6,127.8,124.2,110.3,68.1,63.5,55.6,48.8,42.3,34.1,31.5,29.2,28.2,24.7$, 19.2. Enantiometric excess: 99\%, determined by HPLC (Daicel Chiralpak IE column, hexane/EtOH/DEA 95:5:0.1, flow rate $0.5 \mathrm{~mL} / \mathrm{min}, \mathrm{t}_{\text {major }}=19.55 \mathrm{~min}, \mathrm{t}_{\text {minor }}=22.82 \mathrm{~min}, \lambda=210$ nm). HRMS (ESI-TOF) m/z: [M+H] ${ }^{+}$Calcd. for $\mathrm{C}_{19} \mathrm{H}_{30} \mathrm{NO}_{2}$ 304.2277; Found 304.2285.

\section{(2S,3S)-2-(Benzo[d][1,3]dioxol-5-ylmethyl)quinuclidin-3-ol (2p):}

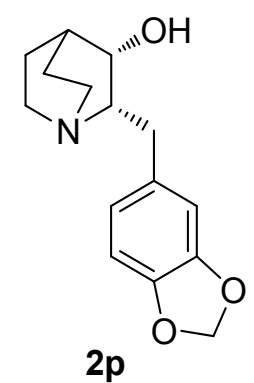

White solid (301 mg, 96\% yield); mp 152-153 ${ }^{\circ} \mathrm{C} ;[\alpha]_{\mathrm{D}}^{26}-68.5,(\mathrm{c}=0.4, \mathrm{MeOH})$. ${ }^{1} \mathrm{H}$ NMR (400 MHz, $\left.\mathrm{CDCl}_{3}\right) \delta 6.84(\mathrm{~s}, 1 \mathrm{H}), 6.80-6.73(\mathrm{~m}, 2 \mathrm{H}), 5.94(\mathrm{~s}, 2 \mathrm{H})$, $4.01-3.80(\mathrm{~m}, 1 \mathrm{H}), 3.23-3.05(\mathrm{~m}, 3 \mathrm{H}), 2.92-2.77(\mathrm{~m}, 3 \mathrm{H}), 2.73(\mathrm{dd}, J=$ $18.9,7.9 \mathrm{~Hz}, 1 \mathrm{H}), 1.93(\mathrm{~s}, 3 \mathrm{H}), 1.69-1.60(\mathrm{~m}, 1 \mathrm{H}), 1.49(\mathrm{dd}, J=20.9,9.9 \mathrm{~Hz}$, 1H), $1.34(\mathrm{dd}, J=10.6,7.2 \mathrm{~Hz}, 1 \mathrm{H}) .{ }^{13} \mathrm{C} \mathrm{NMR}\left(101 \mathrm{MHz}, \mathrm{CDCl}_{3}\right) \delta 147.8$, $145.8,134.1,121.5,109.3,108.3,100.8,69.0,62.0,48.9,41.7,33.4,28.8,24.7,18.9$. Enantiometric excess: 98\%, determined by HPLC (Daicel Chiralpak IE column, hexane $/ \mathrm{EtOH} / \mathrm{DEA}$ 60:40:0.1, flow rate $0.5 \mathrm{~mL} / \mathrm{min}, \mathrm{t}_{\text {major }}=24.49 \mathrm{~min}, \mathrm{t}_{\text {minor }}=12.17 \mathrm{~min}, \lambda=210$ nm). HRMS (ESI-TOF) m/z: [M+H] ${ }^{+}$Calcd. for $\mathrm{C}_{15} \mathrm{H}_{20} \mathrm{NO}_{3}$ 262.1443; Found 262.1445.

\section{(2S,3S)-2-(Naphthalen-1-ylmethyl)quinuclidin-3-ol (2q):}

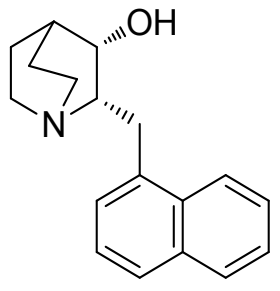

$2 q$

White solid (314 mg, 98\% yield); mp 181-182 ${ }^{\circ} \mathrm{C} ;[\alpha]_{\mathrm{D}}^{26} \quad-92.0,(\mathrm{c}=0.4$, MeOH). ${ }^{1} \mathrm{H}$ NMR (400 MHz, DMSO- $\left.d_{6}\right) \delta 8.09(\mathrm{~d}, J=8.3 \mathrm{~Hz}, 1 \mathrm{H}), 7.89(\mathrm{~d}$, $J=7.7 \mathrm{~Hz}, 1 \mathrm{H}), 7.78-7.66(\mathrm{~m}, 1 \mathrm{H}), 7.60-7.44(\mathrm{~m}, 2 \mathrm{H}), 7.39(\mathrm{~d}, J=5.2$ $\mathrm{Hz}, 2 \mathrm{H}), 4.80(\mathrm{~d}, J=4.1 \mathrm{~Hz}, 1 \mathrm{H}), 3.94-3.79(\mathrm{~m}, 1 \mathrm{H}), 3.46(\mathrm{dd}, J=13.4,3.2$ $\mathrm{Hz}, 1 \mathrm{H}), 3.25-3.00(\mathrm{~m}, 3 \mathrm{H}), 2.66-2.51(\mathrm{~m}, 2 \mathrm{H}), 2.43(\mathrm{dd}, J=12.7,10.4$ $\mathrm{Hz}, 1 \mathrm{H}), 1.98-1.84(\mathrm{~m}, 1 \mathrm{H}), 1.80(\mathrm{~d}, J=1.7 \mathrm{~Hz}, 1 \mathrm{H}), 1.58-1.46(\mathrm{~m}, 1 \mathrm{H}), 1.46-1.30(\mathrm{~m}, 1 \mathrm{H})$, $1.19(\mathrm{dd}, J=18.4,10.9 \mathrm{~Hz}, 1 \mathrm{H}) .{ }^{13} \mathrm{C}$ NMR (101 MHz, DMSO- $\left.d_{6}\right) \delta 138.3,133.8,132.3,129.0$, $127.40,126.3,126.1,125.8,125.6,124.2,68.2,61.9,49.1,41.5,29.6,29.4,25.0,19.6$. Enantiometric excess: $>99 \%$, determined by HPLC (Daicel Chiralpak IE column, hexane/IPA/DEA 85:15:0.1, flow rate $0.5 \mathrm{~mL} / \mathrm{min}, \mathrm{t}_{\text {major }}=22.86 \mathrm{~min}, \mathrm{t}_{\text {minor }}=18.39 \mathrm{~min}, \lambda=210$ nm). HRMS (ESI-TOF) m/z: [M+H] $]^{+}$Calcd. for $\mathrm{C}_{18} \mathrm{H}_{22} \mathrm{NO} 268.1701$; Found 268.1698. 


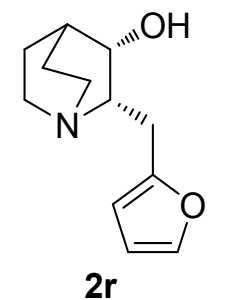

White solid (239 mg, 96\% yield); mp 133-134 ${ }^{\circ} \mathrm{C} ;[\alpha]_{\mathrm{D}}^{26}-101.0,(\mathrm{c}=0.4, \mathrm{MeOH})$. ${ }^{1} \mathrm{H}$ NMR $\left(400 \mathrm{MHz}, \mathrm{CDCl}_{3}\right) \delta 7.34(\mathrm{~d}, J=1.0 \mathrm{~Hz}, 1 \mathrm{H}), 6.33(\mathrm{dd}, J=2.8,1.9 \mathrm{~Hz}$, 1H), $6.12(\mathrm{~d}, J=2.9 \mathrm{~Hz}, 1 \mathrm{H}), 3.93(\mathrm{dd}, J=6.7,4.7 \mathrm{~Hz}, 1 \mathrm{H}), 3.34$ (dd, $J=14.8$, $10.5 \mathrm{~Hz}, 1 \mathrm{H}), 3.10$ (ddd, $J=21.0,13.0,6.9 \mathrm{~Hz}, 2 \mathrm{H}), 2.91-2.78(\mathrm{~m}, 3 \mathrm{H}), 2.78-$ $2.65(\mathrm{~m}, 1 \mathrm{H}), 2.39(\mathrm{~s}, 1 \mathrm{H}), 2.06-1.83(\mathrm{~m}, 2 \mathrm{H}), 1.78-1.60(\mathrm{~m}, 1 \mathrm{H}), 1.57-1.43(\mathrm{~m}, 1 \mathrm{H}), 1.36-$ $1.26(\mathrm{~m}, 1 \mathrm{H}) .{ }^{13} \mathrm{C} \mathrm{NMR}\left(151 \mathrm{MHz}, \mathrm{CDCl}_{3}\right) \delta 154.4,141.2,110.5,106.0,68.6,60.33,48.7,41.8$, 28.3, 26.9, 24.6, 18.8. Enantiometric excess: 97\%, determined by HPLC (Daicel Chiralpak IE column, hexane/EtOH/IPA/DEA 82:9:9:0.1, flow rate $0.5 \mathrm{~mL} / \mathrm{min}, \mathrm{t}_{\text {major }}=11.89 \mathrm{~min}, \mathrm{t}_{\text {minor }}=$ $11.45 \mathrm{~min}, \lambda=210 \mathrm{~nm})$. HRMS ESI-TOF m/z: $[\mathrm{M}+\mathrm{H}]^{+}$Calcd. for $\left(\mathrm{C}_{12} \mathrm{H}_{18} \mathrm{NO}_{2}\right)$ 208.1338; Found 208.1342 .

\section{(2S,3S)-2-((5-Methylthiophen-2-yl)methyl)quinuclidin-3-ol (2s):}

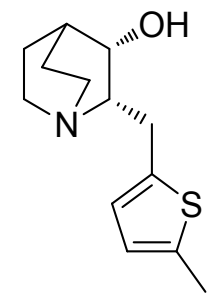

White solid (276 mg, 97\% yield); mp $140-141{ }^{\circ} \mathrm{C} ;[\alpha]_{\mathrm{D}}^{26}-74.5$, (c = 0.4, MeOH). ${ }^{1} \mathrm{H}$ NMR $\left(600 \mathrm{MHz}, \mathrm{CDCl}_{3}\right) \delta 6.69(\mathrm{~d}, J=3.1 \mathrm{~Hz}, 1 \mathrm{H}), 6.58(\mathrm{~d}, J=2.2 \mathrm{~Hz}, 1 \mathrm{H})$, $3.99(\mathrm{dd}, J=7.0,4.6 \mathrm{~Hz}, 1 \mathrm{H}), 3.42(\mathrm{dd}, J=14.8,9.7 \mathrm{~Hz}, 1 \mathrm{H}), 3.19-3.03(\mathrm{~m}$, 2H), $2.94(\mathrm{dd}, J=14.8,5.7 \mathrm{~Hz}, 1 \mathrm{H}), 2.86(\mathrm{t}, J=7.6 \mathrm{~Hz}, 2 \mathrm{H}), 2.79-2.66(\mathrm{~m}, 1 \mathrm{H})$, $2 s$ $2.45(\mathrm{~s}, 3 \mathrm{H}), 1.95-1.85(\mathrm{~m}, 3 \mathrm{H}), 1.74-1.61(\mathrm{~m}, 1 \mathrm{H}), 1.57-1.42(\mathrm{~m}, 1 \mathrm{H}), 1.39-$ $1.21(\mathrm{~m}, 1 \mathrm{H}) .{ }^{13} \mathrm{C}$ NMR $\left(151 \mathrm{MHz}, \mathrm{CDCl}_{3}\right) \delta 141.0,138.2,124.7,124.6,68.7,62.1,48.8,41.8$, 28.7, 28.6, 24.7, 18.9, 15.3. Enantiometric excess: >99\%, determined by HPLC (Daicel Chiralpak IE column, hexane/EtOH/DEA 60:40:0.1, flow rate $0.5 \mathrm{~mL} / \mathrm{min}, \mathrm{t}_{\text {major }}=15.11 \mathrm{~min}, \lambda=210 \mathrm{~nm}$ ). HRMS (ESI-TOF) m/z: [M+H] $]^{+}$Calcd. for $\mathrm{C}_{13} \mathrm{H}_{20} \mathrm{NOS} 238.1266$; Found 238.1264.

\section{(2S,3S)-2-Benzhydrylquinuclidin-3-ol (2t):}

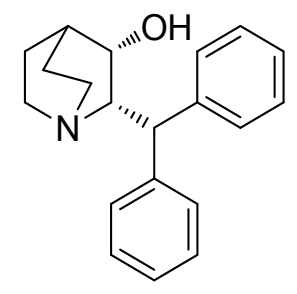

$2 t$

White solid (345 mg, 98\% yield); $\mathrm{mp} 178-179{ }^{\circ} \mathrm{C} ;[\alpha]_{\mathrm{D}}^{26}+2.0,(\mathrm{c}=1.00$, $\mathrm{MeCN})\left(\right.$ lit. $^{12}[\alpha]_{\mathrm{D}}^{26}+11.6\left(\mathrm{c} 1, \mathrm{CDCl}_{3}\right), 99.5 \%$ ee $\left.(\mathrm{S}, \mathrm{S})\right) .{ }^{1} \mathrm{H}$ NMR $(400 \mathrm{MHz}$, DMSO- $\left.d_{6}\right) \delta 8.26(\mathrm{~s}, 1 \mathrm{H}), 7.45(\mathrm{~d}, J=7.4 \mathrm{~Hz}, 2 \mathrm{H}), 7.35(\mathrm{~d}, J=7.4 \mathrm{~Hz}, 2 \mathrm{H})$, $7.22(\mathrm{dt}, J=13.3,7.6 \mathrm{~Hz}, 4 \mathrm{H}), 7.09(\mathrm{dt}, J=10.6,5.4 \mathrm{~Hz}, 2 \mathrm{H}), 4.54(\mathrm{~d}, J=$ $12.1 \mathrm{~Hz}, 1 \mathrm{H}), 4.11-3.94(\mathrm{~m}, 1 \mathrm{H}), 3.81-3.67(\mathrm{~m}, 1 \mathrm{H}), 3.11(\mathrm{dd}, J=20.7$, $9.2 \mathrm{~Hz}, 1 \mathrm{H}), 2.93-2.80(\mathrm{~m}, 1 \mathrm{H}), 2.77-2.62(\mathrm{~m}, 1 \mathrm{H}), 1.97-1.76(\mathrm{~m}, 2 \mathrm{H}), 1.61(\mathrm{~s}, 2 \mathrm{H}), 1.28(\mathrm{dd}$, $J=18.8,9.6 \mathrm{~Hz}, 1 \mathrm{H}) .{ }^{13} \mathrm{C}$ NMR $\left(151 \mathrm{MHz}, \mathrm{DMSO}-d_{6}\right) \delta 164.8,144.8,144.7,128.8,128.7,128.5$, 
$128.16,126.3,126.1,65.9,62.5,55.3,48.8,48.3,41.8,29.2,22.9,18.9$. Enantiometric excess: $>99 \%$, determined by HPLC (Daicel Chiralpak IE column, hexane/EtOH/DEA 80:20:0.1, flow rate $0.5 \mathrm{~mL} / \mathrm{min}, \mathrm{t}_{\text {major }}=112.77 \mathrm{~min}, \lambda=210 \mathrm{~nm}$ ). HRMS (ESI-TOF) $\mathrm{m} / \mathrm{z}:[\mathrm{M}+\mathrm{H}]^{+}$Calcd. for $\mathrm{C}_{20} \mathrm{H}_{24} \mathrm{NO} 294.1858$; Found 294.1863.

\section{(2S,3S)-2-(Bis(4-fluorophenyl)methyl)quinuclidin-3-ol (2u):}

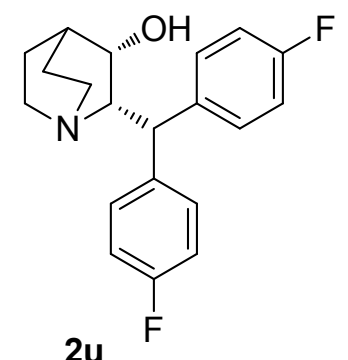

White solid (391 mg, 99\% yield); mp 184-185 ${ }^{\circ} \mathrm{C} ;[\alpha]_{\mathrm{D}}^{26}-9.5,(\mathrm{c}=0.4$, MeOH). ${ }^{1} \mathrm{H}$ NMR (400 MHz, $\left.\mathrm{CDCl}_{3}\right) \delta 7.40(\mathrm{dd}, J=8.6,5.3 \mathrm{~Hz}, 2 \mathrm{H})$, $7.20(\mathrm{dd}, J=8.6,5.4 \mathrm{~Hz}, 2 \mathrm{H}), 7.04(\mathrm{t}, J=8.6 \mathrm{~Hz}, 2 \mathrm{H}), 6.94(\mathrm{t}, J=8.7 \mathrm{~Hz}$, 2H), $4.51(\mathrm{~d}, J=12.0 \mathrm{~Hz}, 1 \mathrm{H}), 4.01-3.88(\mathrm{~m}, 1 \mathrm{H}), 3.54(\mathrm{dd}, J=11.9$, $6.8 \mathrm{~Hz}, 1 \mathrm{H}), 3.32-3.01(\mathrm{~m}, 1 \mathrm{H}), 2.90-2.73(\mathrm{~m}, 2 \mathrm{H}), 2.67(\mathrm{t}, J=11.8$ $\mathrm{Hz}, 1 \mathrm{H}), 1.93(\mathrm{dd}, J=9.8,2.7 \mathrm{~Hz}, 1 \mathrm{H}), 1.72-1.62(\mathrm{~m}, 2 \mathrm{H}), 1.54(\mathrm{dt}, J=12.7,10.1 \mathrm{~Hz}, 1 \mathrm{H}), 1.30$ $(\mathrm{dd}, J=7.4,3.6 \mathrm{~Hz}, 2 \mathrm{H}) .{ }^{13} \mathrm{C} \mathrm{NMR}\left(151 \mathrm{MHz}, \mathrm{CDCl}_{3}\right) \delta 162.3,162.0,160.7,160.4,140.0,140.0$, 139.4, 139.4, 129.1, 129.1, 128.9, 128.9, 116.2, 116.1, 115.5, 115.3, 68.7, 63.5, 49.0, 47.56, 41.6, 28.6, 24.7, 19.1. Enantiometric excess: $>99 \%$, determined by HPLC (Daicel Chiralpak IE column, hexane/EtOH/DEA 90:10:0.1, flow rate $0.5 \mathrm{~mL} / \mathrm{min}, \mathrm{t}_{\text {major }}=14.07 \mathrm{~min}, \mathrm{t}_{\text {minor }}=13.48 \mathrm{~min}, \lambda=210$ nm). HRMS (ESI-TOF) m/z: [M+H] $]^{+}$Calcd. for $\left(\mathrm{C}_{20} \mathrm{H}_{22} \mathrm{~F}_{2} \mathrm{NO}\right) 330.1669$; Found, 330.1674 .

\section{(2S,3S)-2-(Bis(4-methoxyphenyl)methyl)quinuclidin-3-ol (2v):}<smiles>COc1ccc(C(c2ccc(OC)cc2)[C@H]2C3CCN(C3)C2CO)cc1</smiles>

$2 v$

White solid (420 mg, 99\% yield); mp $177-178{ }^{\circ} \mathrm{C} ;[\alpha]_{\mathrm{D}}^{26}-8.0,(\mathrm{c}=0.4$, MeOH). ${ }^{1} \mathrm{H}$ NMR $\left(400 \mathrm{MHz}, \mathrm{CDCl}_{3}\right) \delta 7.36(\mathrm{~d}, J=8.6 \mathrm{~Hz}, 2 \mathrm{H}), 7.17$ $(\mathrm{d}, J=8.6 \mathrm{~Hz}, 2 \mathrm{H}), 6.88(\mathrm{~d}, J=8.6 \mathrm{~Hz}, 2 \mathrm{H}), 6.79(\mathrm{~d}, J=8.6 \mathrm{~Hz}, 2 \mathrm{H})$, $4.40(\mathrm{~d}, J=12.1 \mathrm{~Hz}, 1 \mathrm{H}), 4.01-3.90(\mathrm{~m}, 1 \mathrm{H}), 3.76(\mathrm{~d}, J=18.6 \mathrm{~Hz}$, $6 \mathrm{H}), 3.61-3.48(\mathrm{~m}, 1 \mathrm{H}), 3.31-3.08(\mathrm{~m}, 1 \mathrm{H}), 2.91-2.73(\mathrm{~m}, 2 \mathrm{H})$, $2.68(\mathrm{t}, J=11.8 \mathrm{~Hz}, 1 \mathrm{H}), 2.08-1.82(\mathrm{~m}, 2 \mathrm{H}), 1.75-1.61(\mathrm{~m}, 2 \mathrm{H})$, $1.54(\mathrm{dt}, J=12.5,10.0 \mathrm{~Hz}, 1 \mathrm{H}), 1.38-1.15(\mathrm{~m}, 1 \mathrm{H}) .{ }^{13} \mathrm{C} \mathrm{NMR}\left(151 \mathrm{MHz}, \mathrm{CDCl}_{3}\right) \delta$ 158.3, 157.7, $136.9,135.9,128.5,128.3,114.8,114.0,69.0,63.6,55.3,55.1,49.1,47.4,41.6,28.4,24.8,19.2$. Enantiometric excess: $>99 \%$, determined by HPLC (Daicel Chiralpak IE column, hexane/EtOH/DEA 60:40:0.1, flow rate $0.5 \mathrm{~mL} / \mathrm{min}, \mathrm{t}_{\text {major }}=19.34 \mathrm{~min}, \lambda=210 \mathrm{~nm}$ ). HRMS (ESI-TOF) m/z: $[\mathrm{M}+\mathrm{H}]^{+}$Calcd. for $\mathrm{C}_{22} \mathrm{H}_{28} \mathrm{NO}_{3}$ 354.2069; Found 354.2071. 
(2S,3S)-2-(Di(naphthalen-1-yl)methyl)quinuclidin-3-ol (2w):<smiles>O[C@@H](C(c1cccc2ccccc12)c1cccc2ccccc12)C1CC2CCN1C2</smiles>

2w

White solid (458 mg, 98\% yield); mp 343-344 ${ }^{\circ} \mathrm{C} ;[\alpha]_{\mathrm{D}}^{26}+68.0$, $(\mathrm{c}=0.4$, MeOH). ${ }^{1} \mathrm{H}$ NMR $\left(400 \mathrm{MHz}, \mathrm{CDCl}_{3}\right) \delta 8.77(\mathrm{~d}, J=8.6 \mathrm{~Hz}, 1 \mathrm{H}), 8.50-$ $8.30(\mathrm{~m}, 1 \mathrm{H}), 7.95(\mathrm{~d}, J=7.2 \mathrm{~Hz}, 1 \mathrm{H}), 7.87-7.73(\mathrm{~m}, 3 \mathrm{H}), 7.72-7.51$ (m, 4H), $7.48(\mathrm{t}, J=7.3 \mathrm{~Hz}, 1 \mathrm{H}), 7.43-7.36(\mathrm{~m}, 2 \mathrm{H}), 7.33(\mathrm{t}, J=7.7 \mathrm{~Hz}$, $1 \mathrm{H}), 6.14(\mathrm{~d}, J=11.4 \mathrm{~Hz}, 1 \mathrm{H}), 4.22(\mathrm{~s}, 1 \mathrm{H}), 3.87(\mathrm{dd}, J=11.0,6.5 \mathrm{~Hz}$, $1 \mathrm{H}), 3.62(\mathrm{dd}, J=22.1,8.8 \mathrm{~Hz}, 1 \mathrm{H}), 2.76-2.59(\mathrm{~m}, 3 \mathrm{H}), 2.00(\mathrm{dd}, J=9.8,2.3 \mathrm{~Hz}, 1 \mathrm{H}), 1.76-$ $1.59(\mathrm{~m}, 2 \mathrm{H}), 1.32(\mathrm{t}, J=5.3 \mathrm{~Hz}, 1 \mathrm{H}), 1.27(\mathrm{~d}, J=12.3 \mathrm{~Hz}, 1 \mathrm{H}), 1.22(\mathrm{~d}, J=3.1 \mathrm{~Hz}, 1 \mathrm{H}) .{ }^{13} \mathrm{C}$ NMR $\left(151 \mathrm{MHz}, \mathrm{CDCl}_{3}\right) \delta 140.8,139.3,134.3,133.9,132.5,131.8,129.4,128.9,127.6,126.7$, $126.5,125.8,125.7,125.5,125.4,125.3,125.0,124.4,124.1,123.6,69.2,49.3,42.9,28.5,24.6$, 19.4. Enantiometric excess: $98 \%$, determined by HPLC (Gemini-NX column, hexane/EtOH/DEA 70:30:0.1, flow rate $0.5 \mathrm{~mL} / \mathrm{min}, \mathrm{t}_{\text {major }}=9.42 \mathrm{~min}, \mathrm{t}_{\text {minor }}=11.50 \mathrm{~min}, \lambda=210 \mathrm{~nm}$ ). HRMS (ESI-TOF) m/z: [M+H] $]^{+}$Calcd. for $\mathrm{C}_{28} \mathrm{H}_{28} \mathrm{NO}$ 394.2171; Found 394.2171.

\section{Procedures for gram-scale ATH of 1a}

To a mixture of ammonium formate $(6.0 \mathrm{mmol})$ in IPA $(8 \mathrm{~mL})$ and $\mathrm{CH}_{2} \mathrm{Cl}_{2}(8 \mathrm{~mL})$, were added substrate $\mathbf{1}(1.2 \mathrm{mmol})$ and $(R, R)-\mathbf{C 3}(0.2 \mathrm{~mol} \%)$. The mixture was then stirred at $50{ }^{\circ} \mathrm{C}$ in an oil bath for $72 \mathrm{~h}$. After completion of the reaction, the solvents were removed under vacuum. The crude product was dissolved in EA and washed with aqueous $\mathrm{NaHCO}_{3}$ solution. The organic phase was concentrated in vacuo and the resulting mixture was subjected to chiral HPLC analysis to determine the diastereomeric ratio and enantiomeric excess. The crude mixture was finally purified by recrystallization from IPA/hexance (1:6) to give the desired product $\mathbf{2 a}(0.88 \mathrm{~g}, 86 \%$ yield). 


\section{References}

(1) Warawa, E. J.; Mueller, N. J.; Jules, R. Quinuclidine chemistry. 2. Synthesis and antiinflammatory properties of 2-substituted benzhydryl-3-quinuclidinols. J. Med. Chem. 1974, 17, 497-501.

(2) Nugent, T. C.; Seemayer, R. An efficient enantiopure synthesis of a pivotal precursor to Substance P antagonists. Org. Process Res. Dev. 2006, 10, 142-148.

(3) Swain, C. J.; Sewart, E. M.; Cascieri, M. A.; Fong, T. M.; Herbert, R.; MacIntyre, D. E.; Merchant, K. J.; Owen, S. N.; Owens, A. P., Identification of a series of 3-(benzyloxy)-1-azabicyclo[2.2.2] octane human NK1 antagonists. J. Med. Chem. 1995, 38, 4793-4805.

(4) Mazurov, A.; Klucik, J.; Miao, L.; Phillips, T. Y.; Seamans, A.; Schmitt, J. D.; Hauser, T. A.; Johnson, R. T.; Miller, C., 2-(Arylmethyl)-3-substituted quinuclidines as selective $\alpha 7$ nicotinic receptor ligands. Bioorg. Med. Chem. Lett. 2005, 15, 2073-2077.

(5) Bondarenko, V. A.; Trubitsyna, T. K.; Anisimova, O. S.; Mikhlina, E. E.; Mashkovskii, M. D.; Yakhontov, L. N. Synthesis and pharmacological properties of 2-substituted 3-aryl-3-hydroxyquinuclidines. Khimiko-Farmatsevticheskii Zhurnal. 1982, 16, 307-311.

(6) Yanina, A. D.; Vorob'va, V. Ya.; Trubitsyna, T. K.; Mikhlina, E. E.; Mashkovskii, M. D.; Yakhontov, L. N. Synthesis and pharmacological properties of 2-benzylquinuclidine analogs of dopamine. Khimiko-Farmatsevticheskii Zhurnal. 1986, 20, 1202-1206.

(7) Aretz, J.; Anumala, U. R.; Fuchsberger, F. F.; Molavi, N.; Ziebart, N.; Zhang, H.; Nazare, M.; Rademacher, C. J. Am. Chem. Soc. 2018, 140, 14915-14925.

(8) Brown, G. R.; Mallion, K. B.; Harrison, P. J. Di. PCT Int. Appl. 1993, WO 9321184 A1 19931028.

(9) Warawa, E. J.; Campbell, John R. Quinuclidine chemistry. I. Configuration and chemistry of 2-substituted benzylidene-3-quinuclidinones. J. Org. Chem. 1974, 39, 3511-3516.

(10) Yanina, A. D.; Trubitsyna, T. K.; Mikhlina, E. E.; Yakhontov, L. N. Synthesis and pharmacological properties of 2- and 2,3-substituted quinuclidines. Khimiko-Farmatsevticheskii Zhurnal 1987, 21, 808-811.

(11) Kolb, H. C.; Andersson, P. G.; Sharpless, K. B. Toward an understanding of the high enantioselectivity in the osmium-catalyzed asymmetric dihydroxylation (AD). 1. Kinetics. J. Am. Chem. Soc. 1994, 116, 1278-1291.

(12) Arai, N.; Akashi, M.; Sugizaki, S.; Ooka, H.; Inoue, T.; Ohkuma, T. Asymmetric hydrogenation of bicyclic ketones catalyzed by BINAP/IPHAN-Ru (II) complex. Org. Lett. 2010, 12, 3380-3383. 
7. NMR spectra for 1a-w

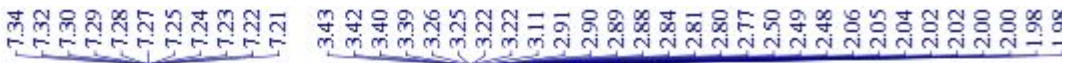
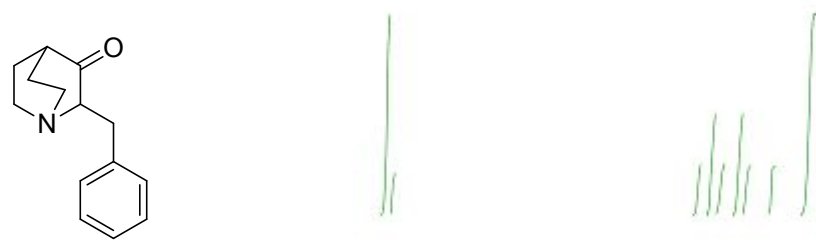

$1 \mathrm{a}$

${ }^{1} \mathrm{H} \mathrm{NMR}\left(400 \mathrm{MHz}, \mathrm{CDCl}_{3}\right)$

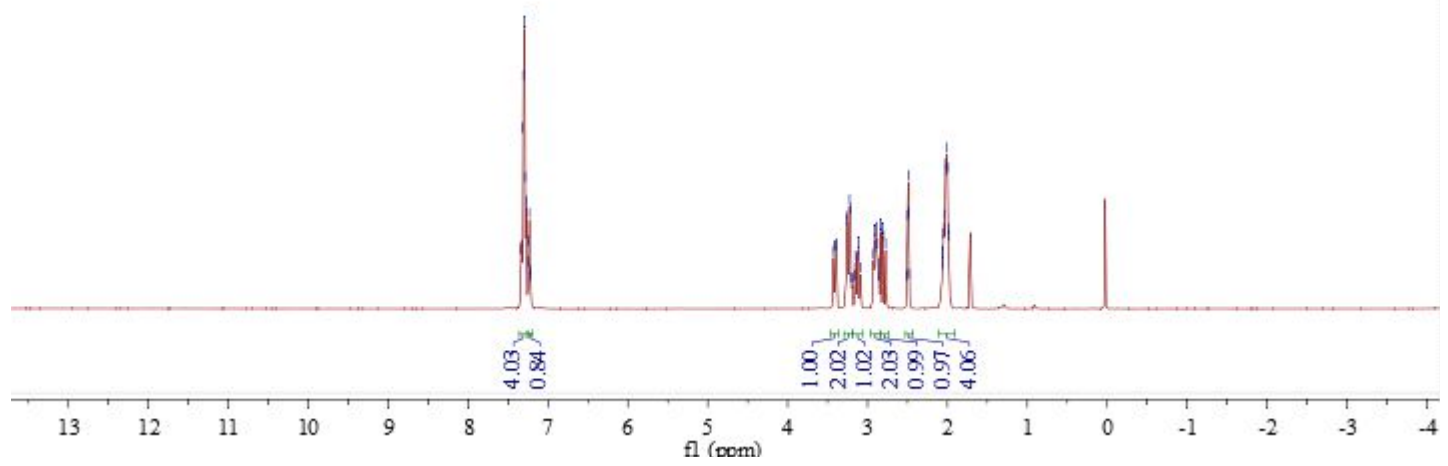

สู่

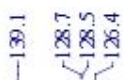

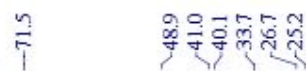

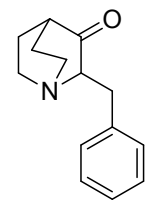

1a

${ }^{13} \mathrm{C} \mathrm{NMR}\left(101 \mathrm{MHz}, \mathrm{CDCl}_{3}\right)$
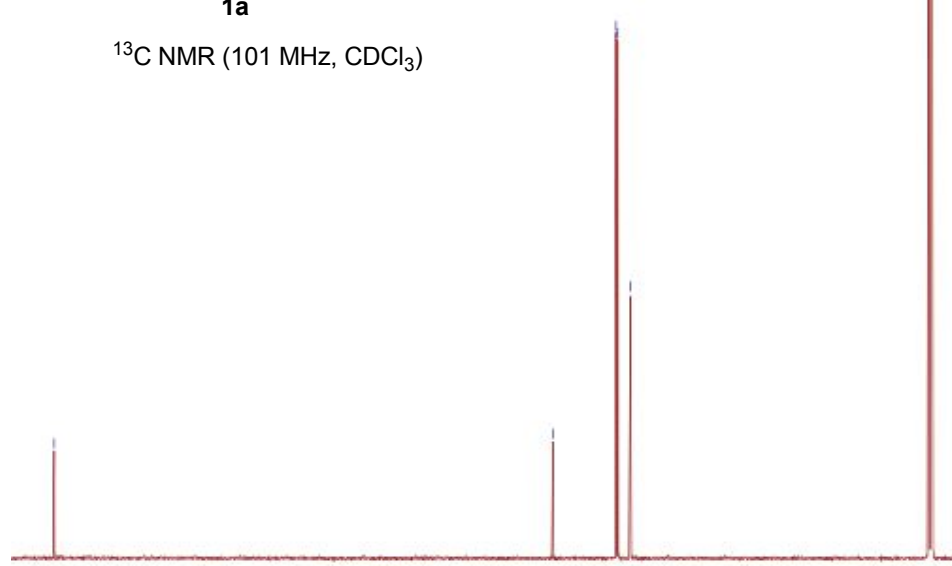

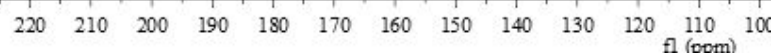




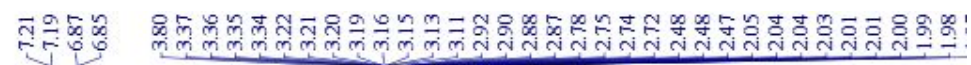
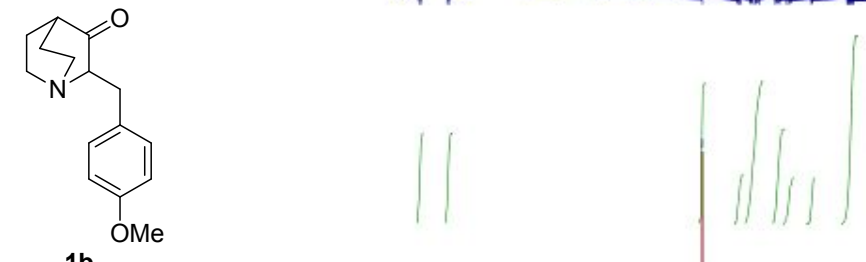

${ }^{1} \mathrm{H}$ NMR (400 MHz, $\mathrm{CDCl}_{3}$ )

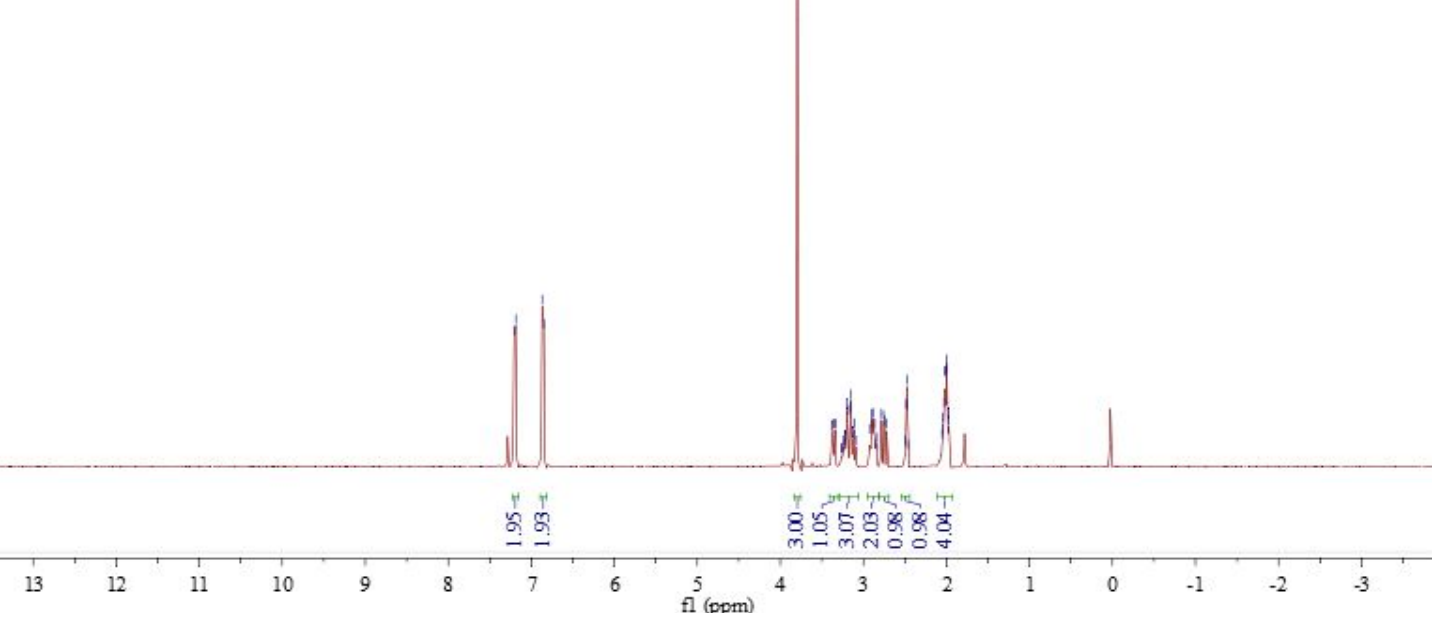

$\stackrel{m}{\stackrel{m}{1}}$
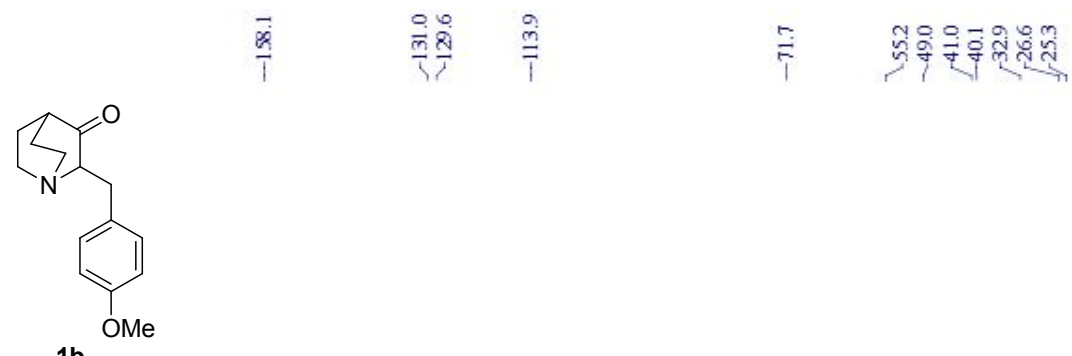

$1 b$

${ }^{13} \mathrm{C}$ NMR (151 MHz, CDCl 3 )

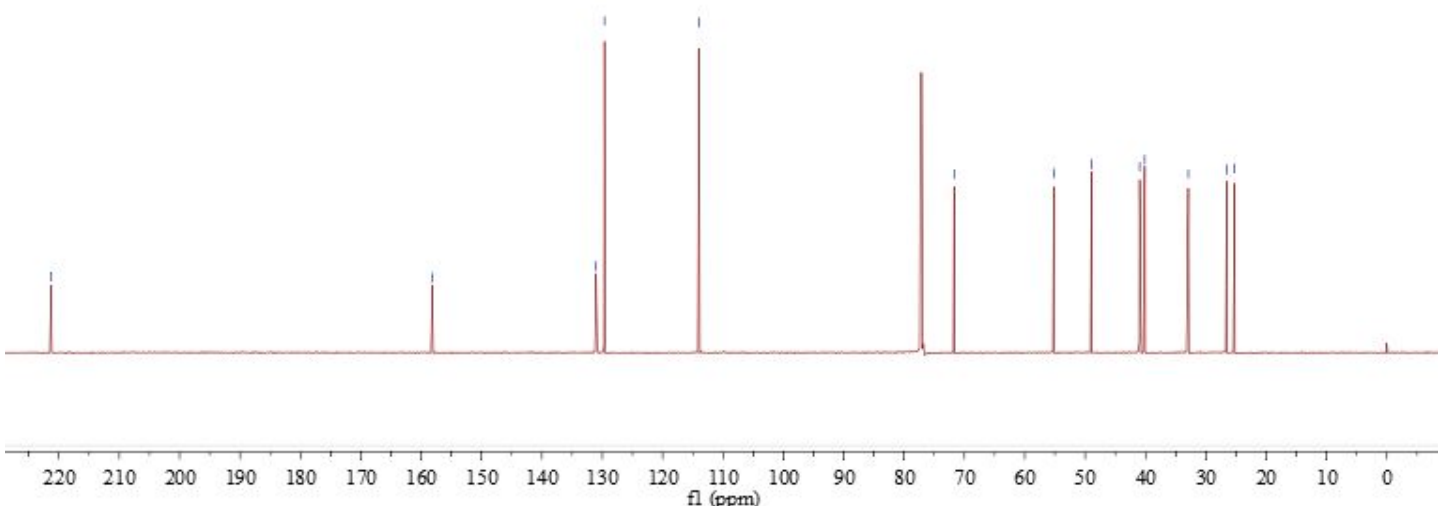




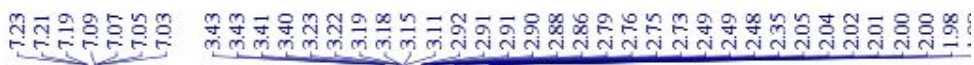

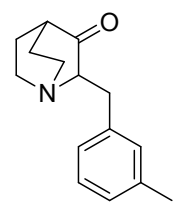

1c

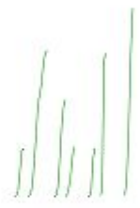

${ }^{1} \mathrm{H}$ NMR $\left(400 \mathrm{MHz}, \mathrm{CDCl}_{3}\right)$

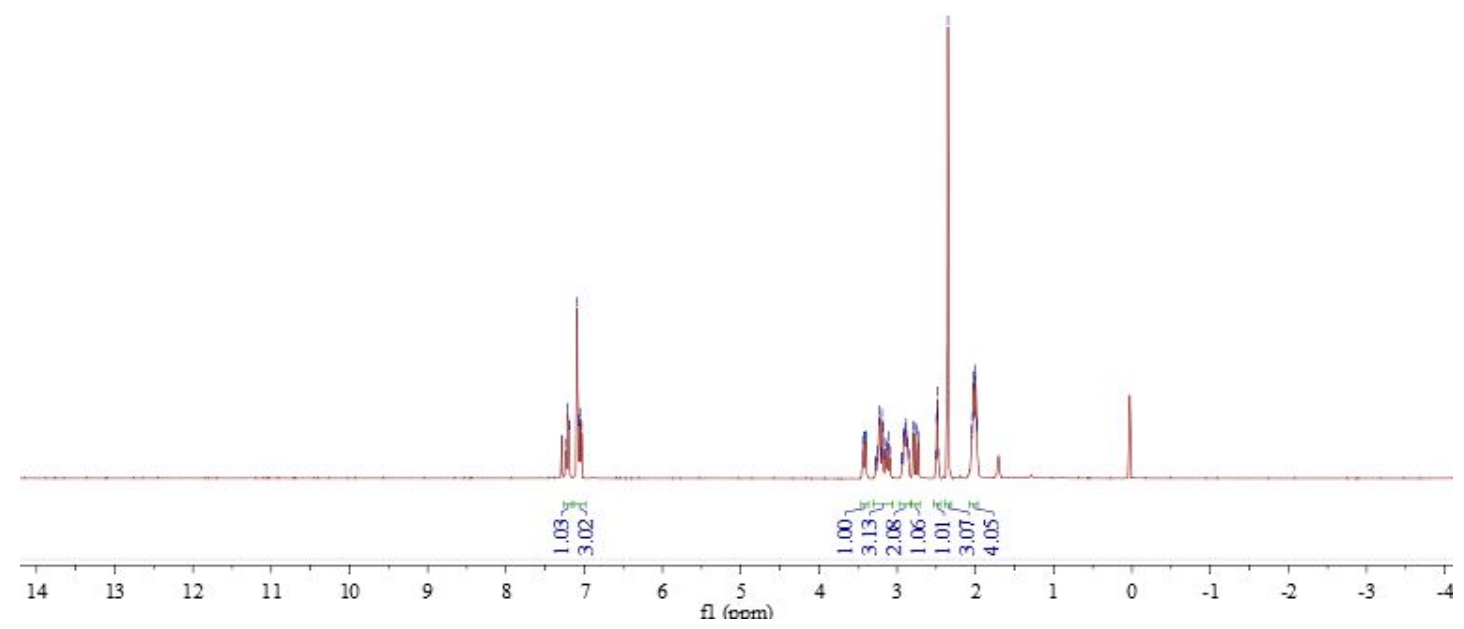

$\stackrel{m}{i}$

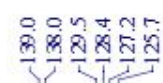

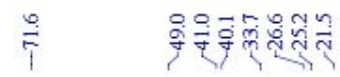

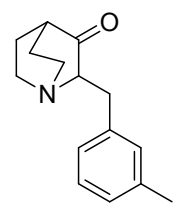

1c

${ }^{13} \mathrm{C}$ NMR $\left(151 \mathrm{MHz}, \mathrm{CDCl}_{3}\right)$

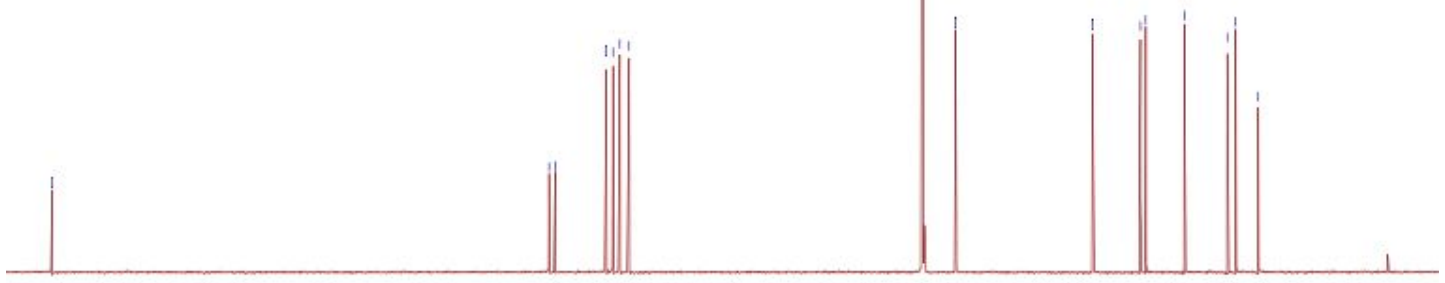

$\begin{array}{llllllllllllllllllllllllllll}220 & 210 & 200 & 190 & 180 & 170 & 160 & 150 & 140 & 130 & 120 & 110 & 100 & 90 & 80 & 70 & 60 & 50 & 40 & 30 & 20 & 10 & 0\end{array}$ 


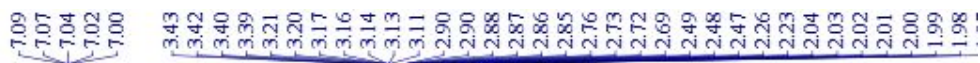

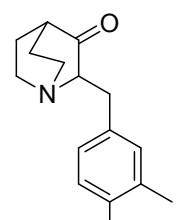

1d

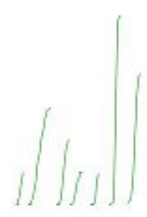

${ }^{1} \mathrm{H} \mathrm{NMR}\left(400 \mathrm{MHz}, \mathrm{CDCl}_{3}\right)$

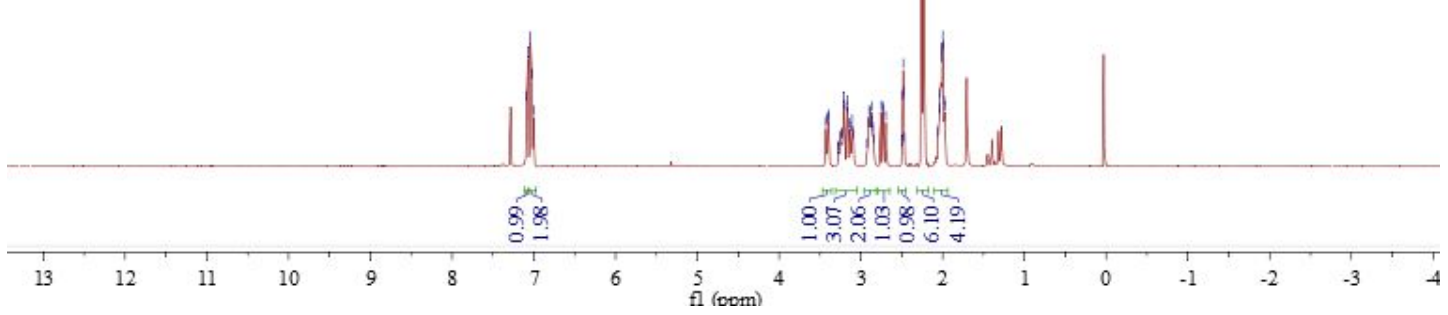

守

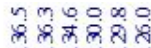

$\frac{0}{1} \frac{0}{2} \frac{9}{3}$

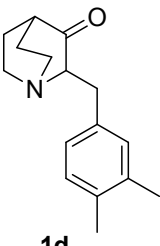

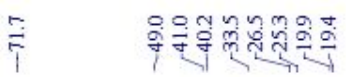

${ }^{13} \mathrm{C}$ NMR $\left(151 \mathrm{MHz}, \mathrm{CDCl}_{3}\right)$

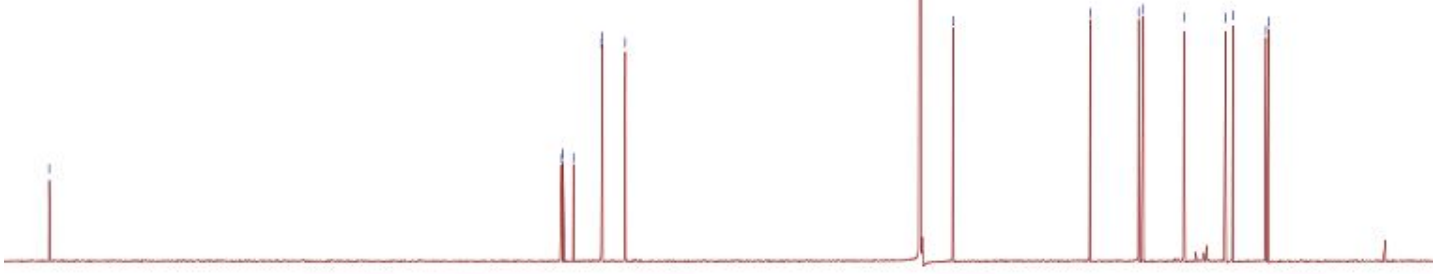

$\begin{array}{lllllllllllllllllllllllllllllllllll}220 & 210 & 200 & 190 & 180 & 170 & 160 & 150 & 140 & 130 & 120 & 110 & 100 & 90 & 80 & 70 & 60 & 50 & 40 & 30 & 20 & 10 & 0\end{array}$ 


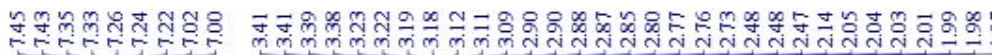

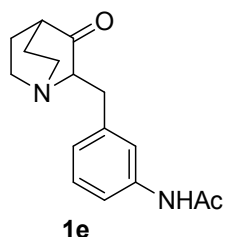

${ }^{1} \mathrm{H}$ NMR $\left(400 \mathrm{MHz}, \mathrm{CDCl}_{3}\right)$
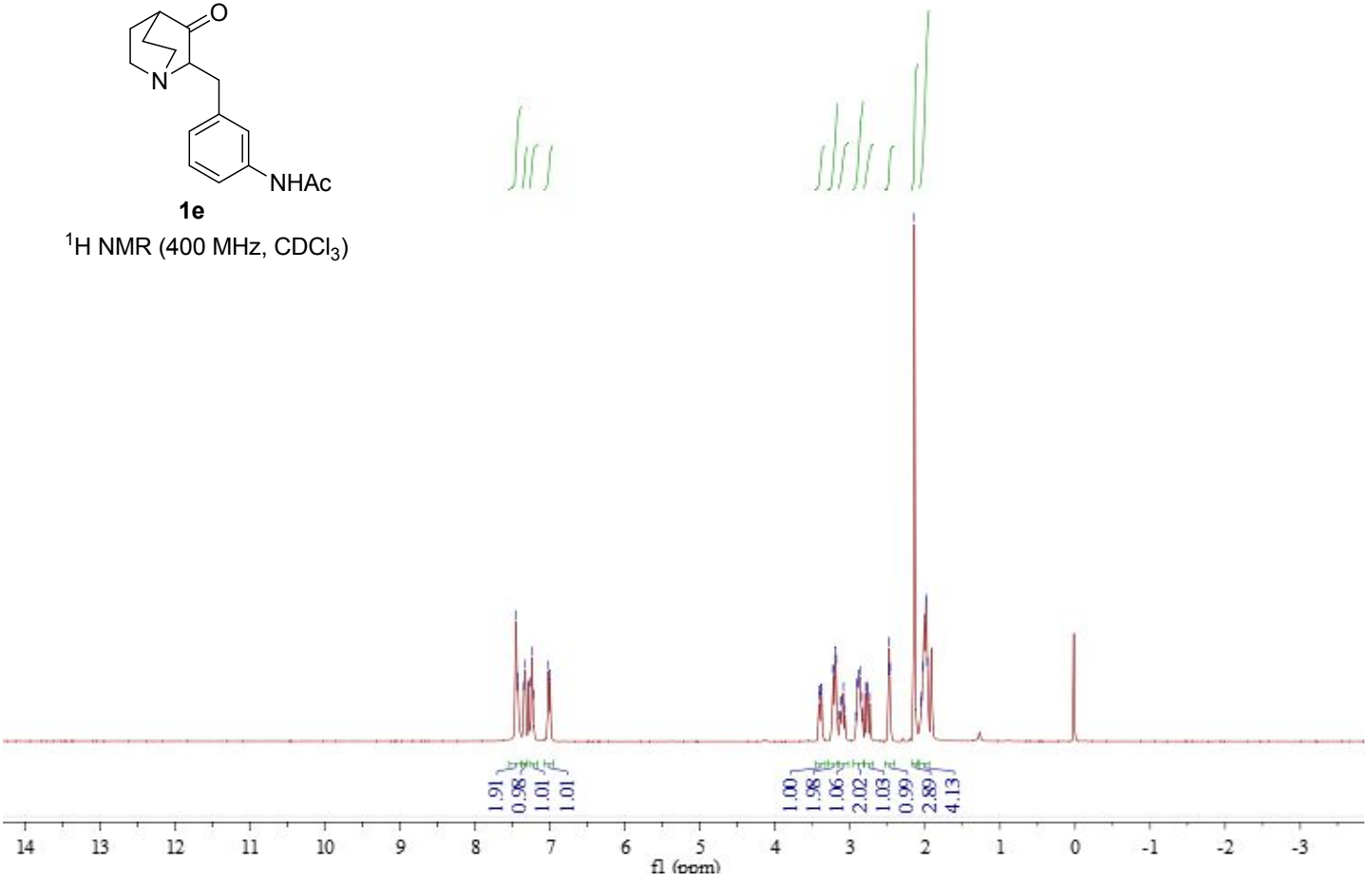

$\stackrel{\text { กิ่ }}{1}$

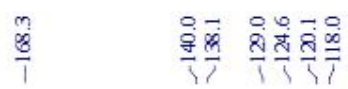

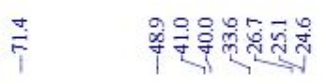

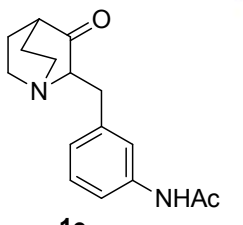

${ }^{13} \mathrm{C}$ NMR $\left(101 \mathrm{MHz}, \mathrm{CDCl}_{3}\right)$

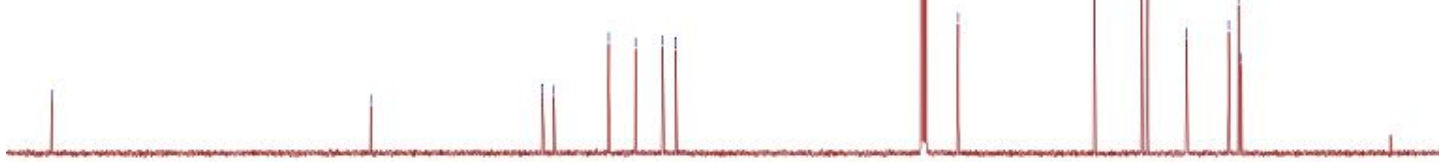

$\begin{array}{lllllllllllllllllllllll}220 & 210 & 200 & 190 & 180 & 170 & 160 & 150 & 140 & 130 & 120 & 110 & 100 & 90 & 80 & 70 & 60 & 50 & 40 & 30 & 20 & 10 & 0\end{array}$ 


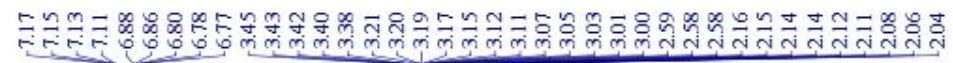

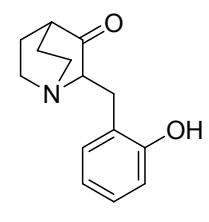

$1 f$

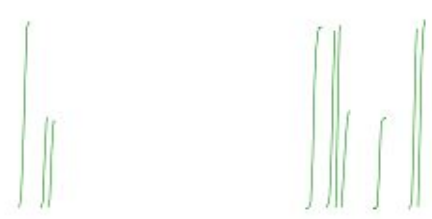

${ }^{1} \mathrm{H} \mathrm{NMR}\left(400 \mathrm{MHz}, \mathrm{CDCl}_{3}\right)$

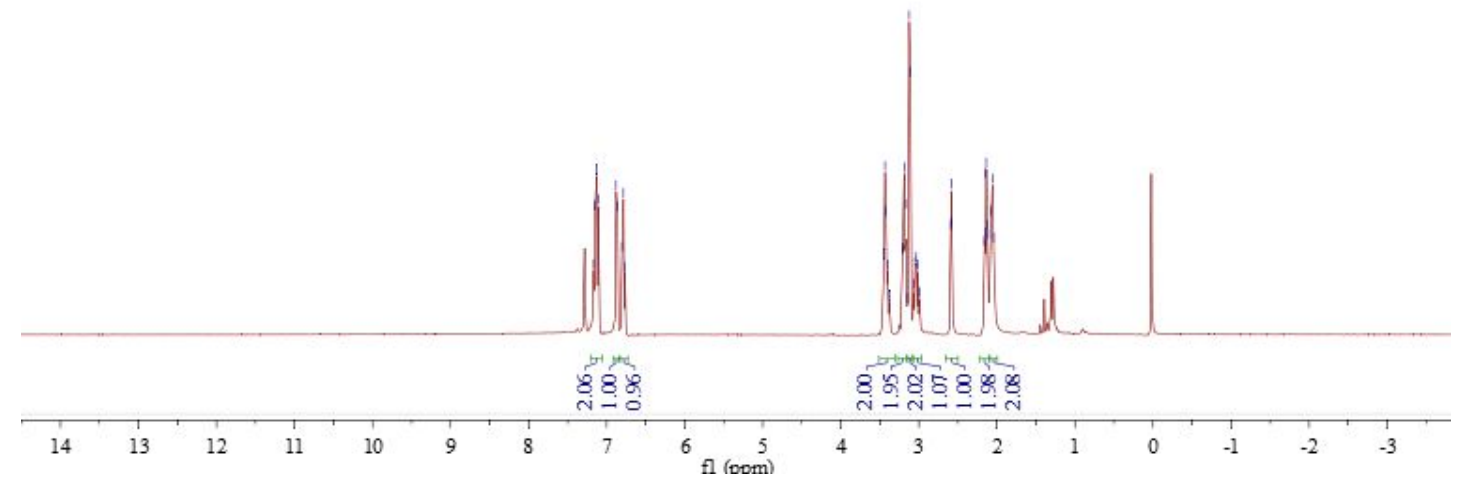

$\stackrel{\text { Tั }}{\text { กั }}$

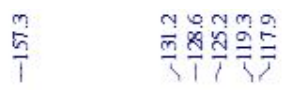

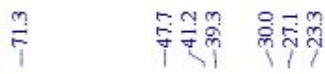

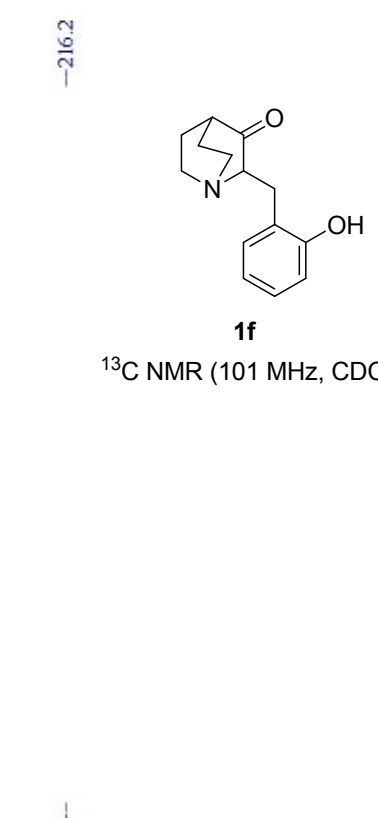

1f

${ }^{13} \mathrm{C}$ NMR $\left(101 \mathrm{MHz}, \mathrm{CDCl}_{3}\right)$

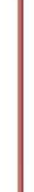

) 


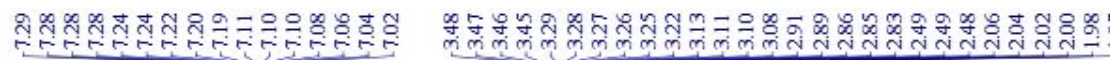
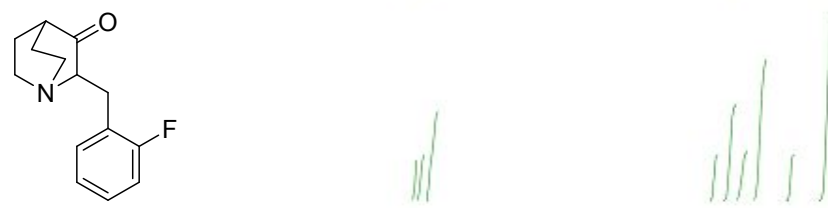

$1 \mathrm{~g}$

${ }^{1} \mathrm{H} \mathrm{NMR}\left(400 \mathrm{MHz}, \mathrm{CDCl}_{3}\right)$

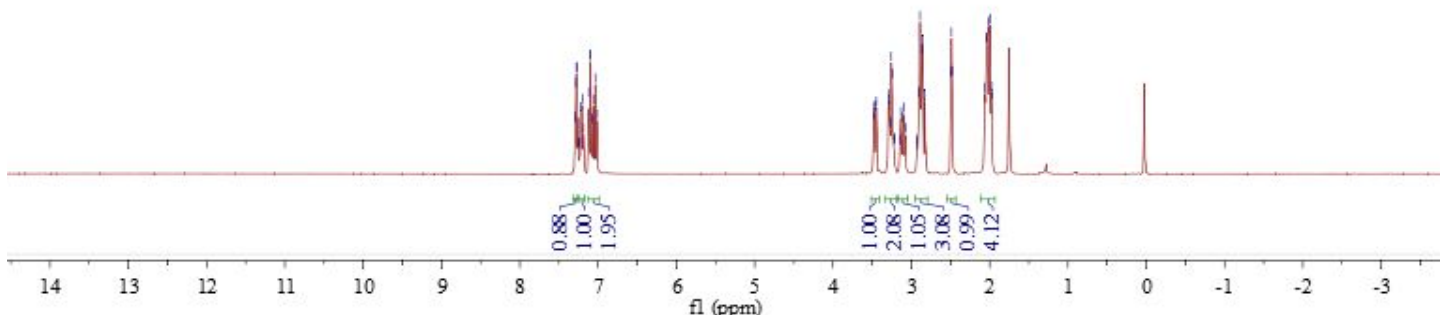

$\underset{\text { ๙ิ }}{\infty}$

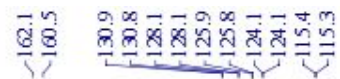

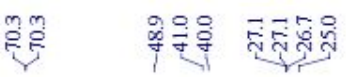<smiles>O=C1C2CCN(C2)C1Cc1ccccc1F</smiles>

$1 \mathrm{~g}$

${ }^{13} \mathrm{C}$ NMR $\left(151 \mathrm{MHz}, \mathrm{CDCl}_{3}\right)$

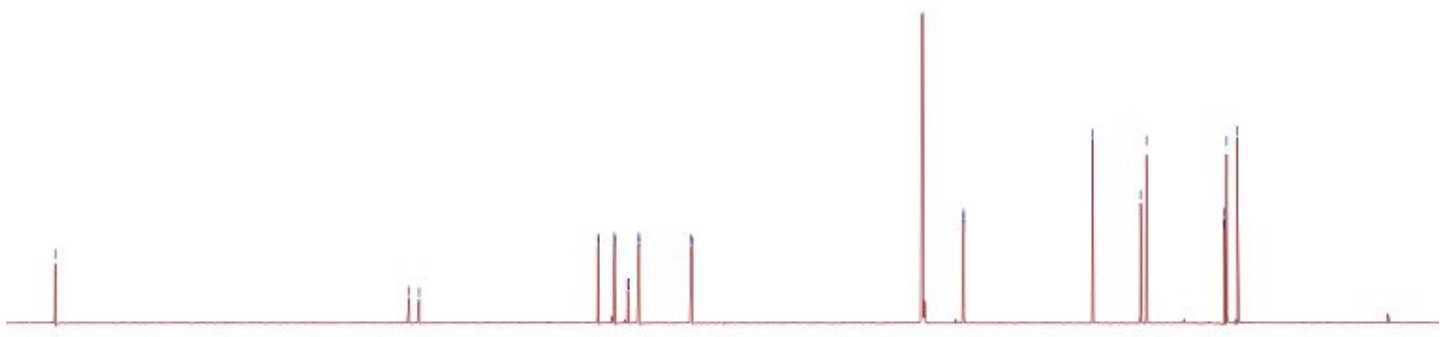

$\begin{array}{lllllllllllllllllllllll}220 & 210 & 200 & 190 & 180 & 170 & 160 & 150 & 140 & 130 & 120 & 110 & 100 & 90 & 80 & 70 & 60 & 50 & 40 & 30 & 20 & 10 & 0\end{array}$ 


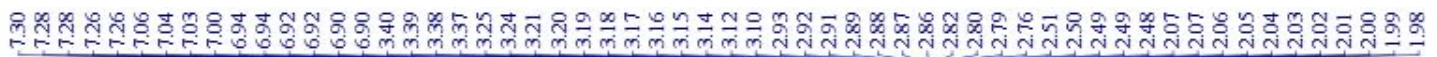<smiles>O=C1C2CCN(C2)C1Cc1cccc(F)c1</smiles>

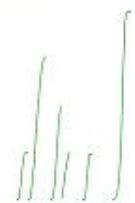

$1 \mathrm{~h}$

${ }^{1} \mathrm{H} \mathrm{NMR}\left(400 \mathrm{MHz}, \mathrm{CDCl}_{3}\right)$

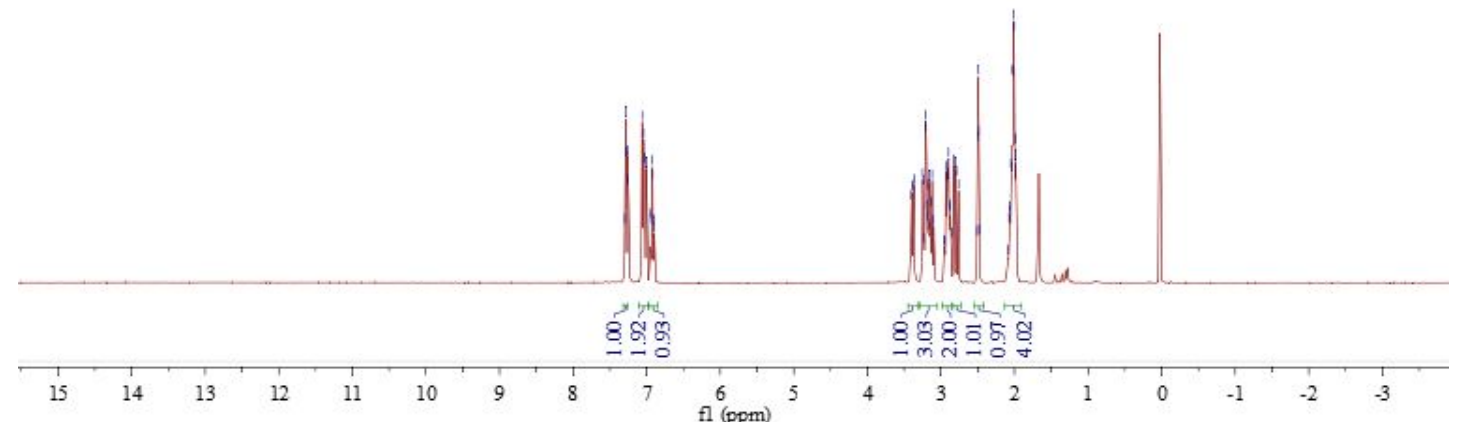

ฉิ่

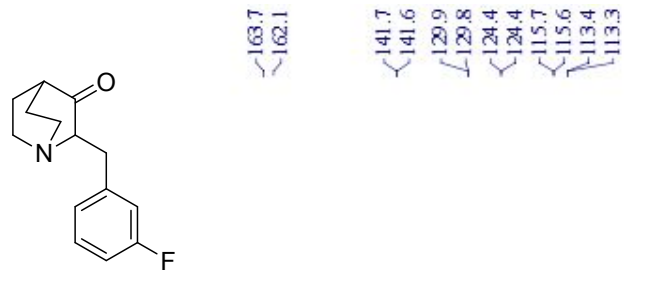

1h

${ }^{13} \mathrm{C}$ NMR $\left(151 \mathrm{MHz}, \mathrm{CDCl}_{3}\right)$

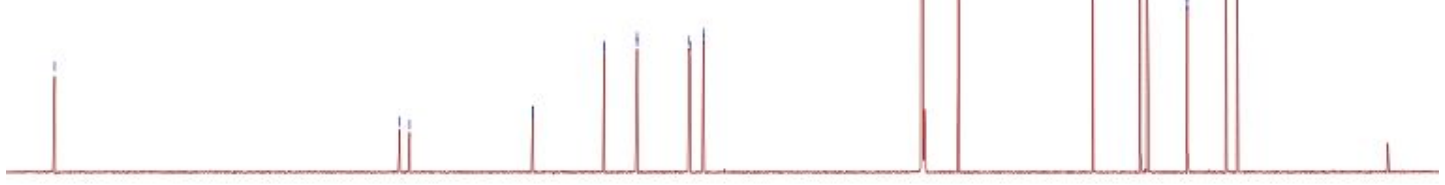

$\begin{array}{llllllllllllllllllllllll}220 & 210 & 200 & 190 & 180 & 170 & 160 & 150 & 140 & 130 & 120 & 110 & 100 & 90 & 80 & 70 & 60 & 50 & 40 & 30 & 20 & 10 & 0\end{array}$ 


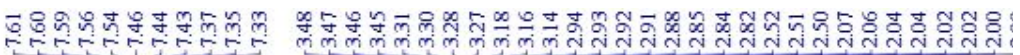
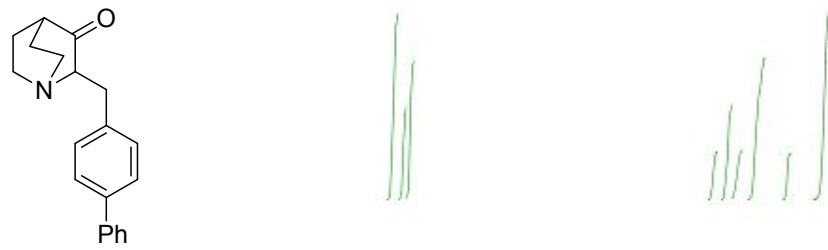

${ }^{1} \mathrm{H}$ NMR $\left(400 \mathrm{MHz}, \mathrm{CDCl}_{3}\right)$

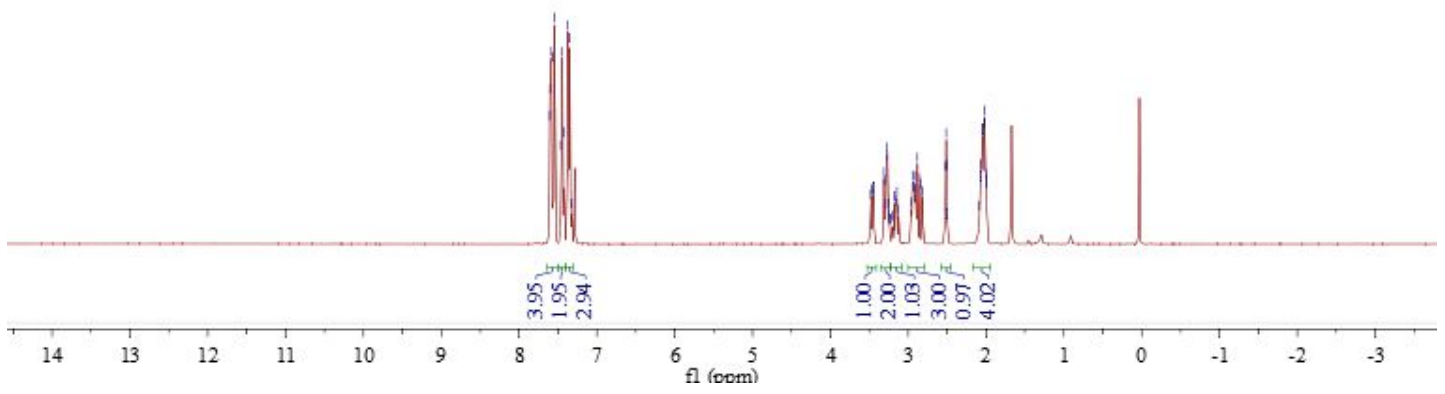

ฟึ่

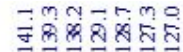

辛

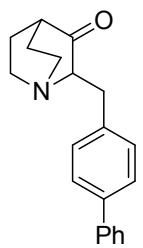

$1 \mathrm{i}$

13C NMR (101 MHz, $\left.\mathrm{CDCl}_{3}\right)$

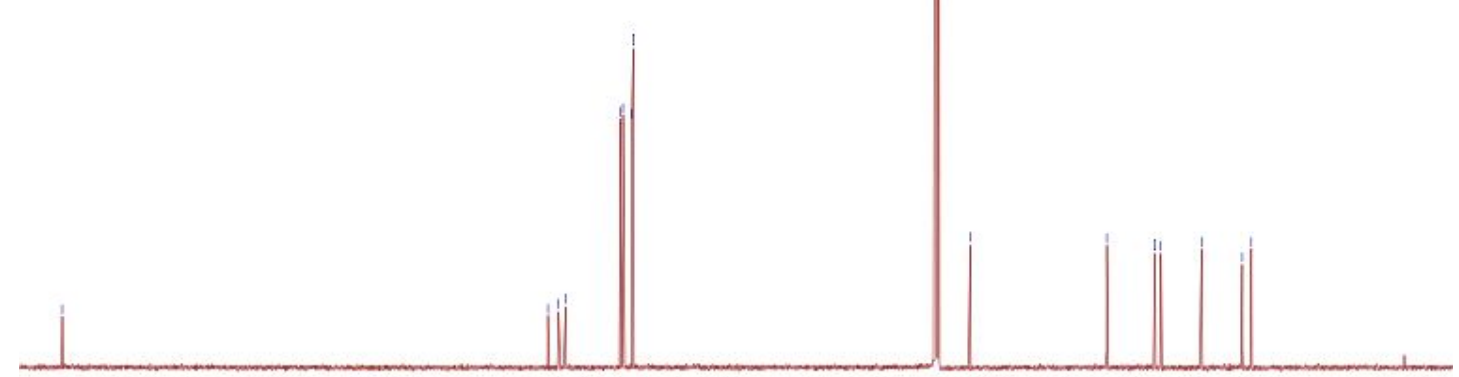

$\begin{array}{lllllllllllllllllllllll}220 & 210 & 200 & 190 & 180 & 170 & 160 & 150 & 140 & 130 & 120 & 110 & 100 & 90 & 80 & 70 & 60 & 50 & 40 & 30 & 20 & 10 & 0\end{array}$ 


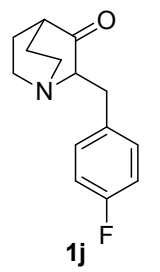

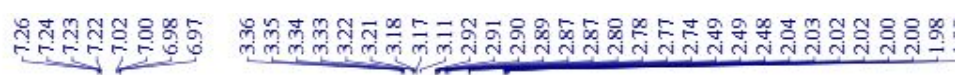

${ }^{1} \mathrm{H}$ NMR $\left(400 \mathrm{MHz}, \mathrm{CDCl}_{3}\right)$
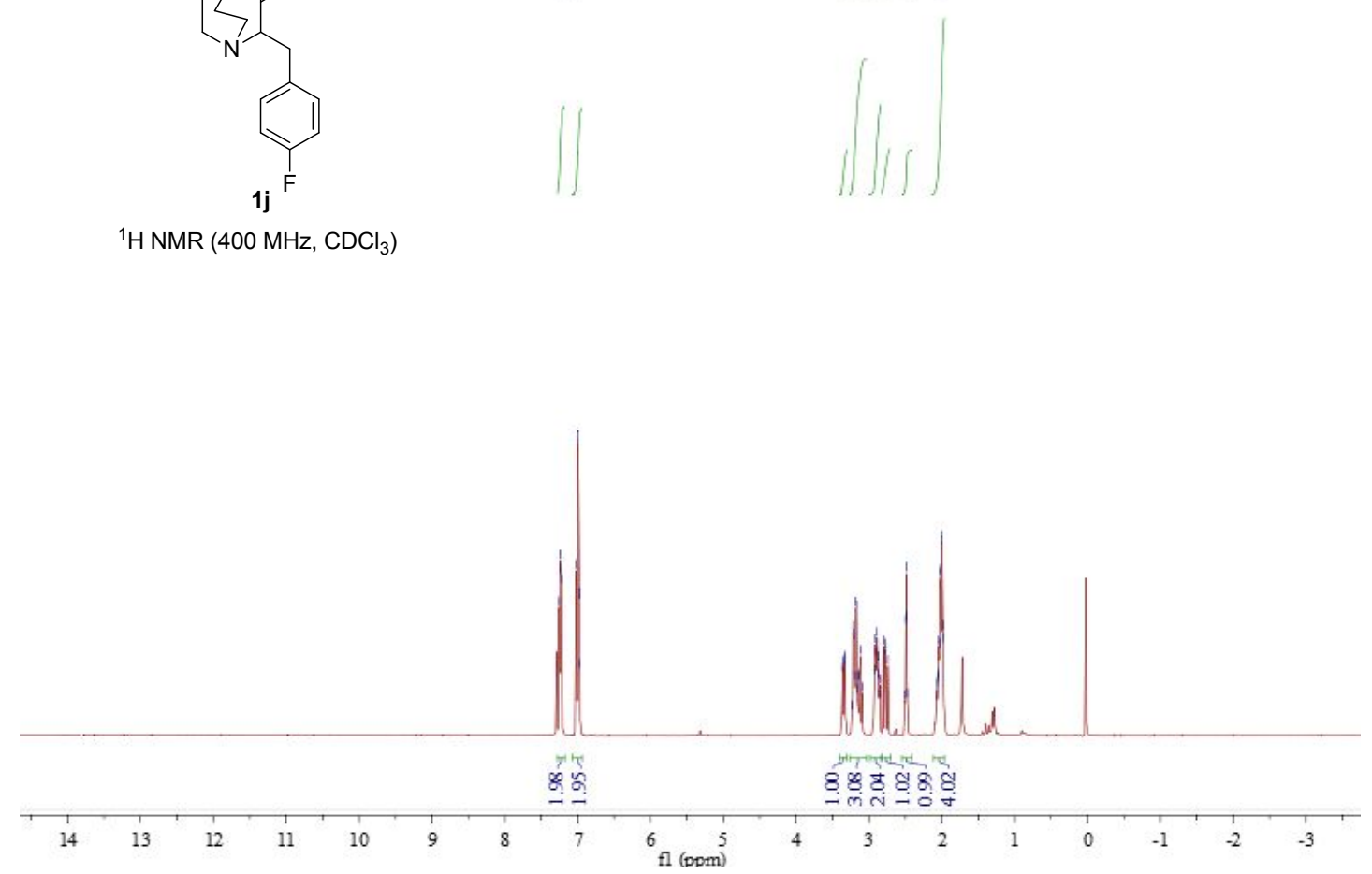

ชิ

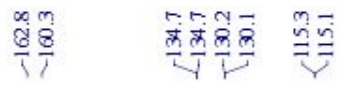

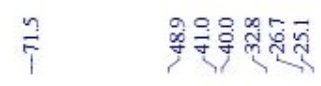

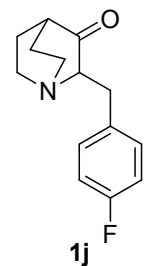

${ }^{13} \mathrm{C}$ NMR $\left(101 \mathrm{MHz}, \mathrm{CDCl}_{3}\right)$

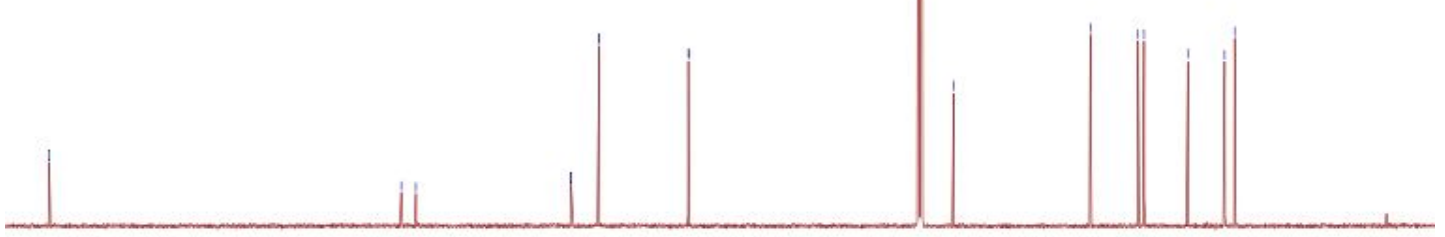

$\begin{array}{lllllllllllllllllllllll}220 & 210 & 200 & 190 & 180 & 170 & 160 & 150 & 140 & 130 & 120 & 110 & 100 & 90 & 80 & 70 & 60 & 50 & 40 & 30 & 20 & 10 & 0\end{array}$ 


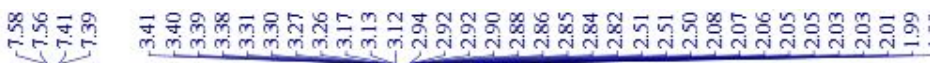
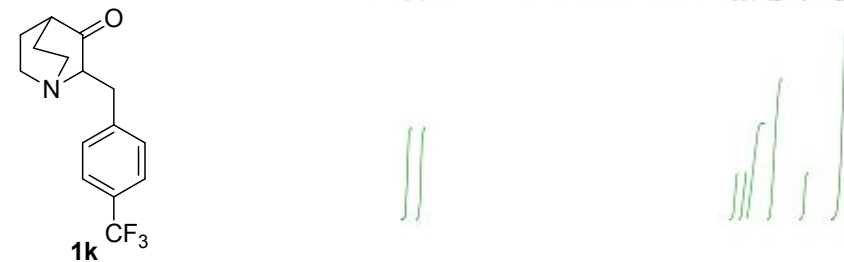

${ }^{1} \mathrm{H}$ NMR $\left(400 \mathrm{MHz}, \mathrm{CDCl}_{3}\right)$

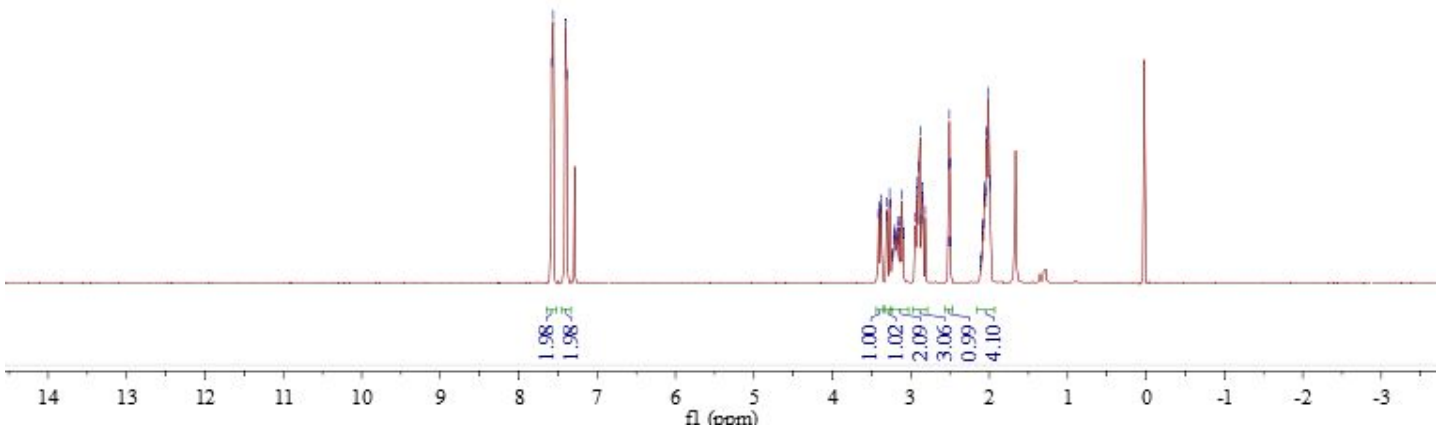

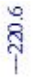
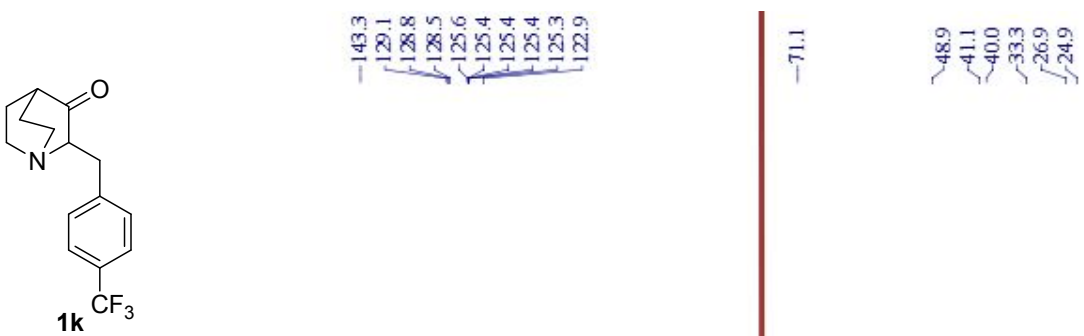

${ }^{13} \mathrm{C}$ NMR $\left(101 \mathrm{MHz}, \mathrm{CDCl}_{3}\right)$

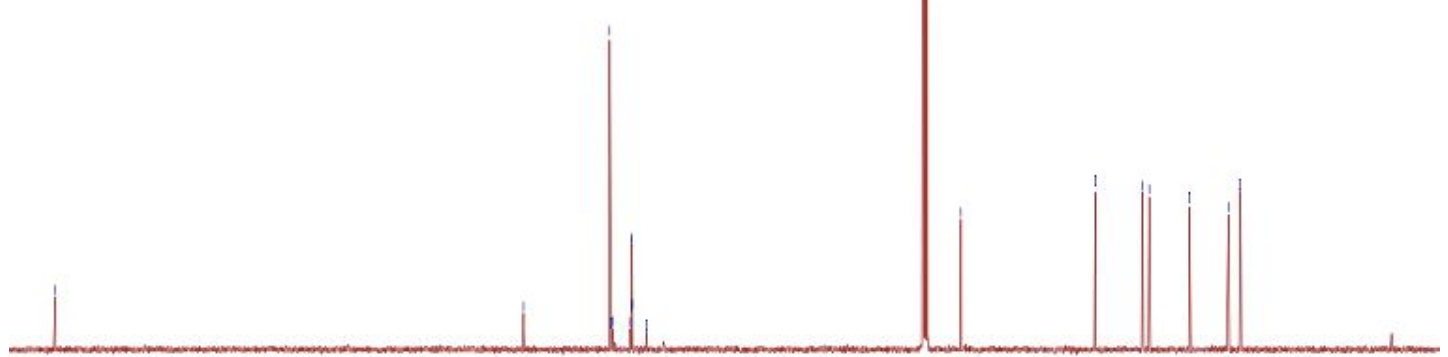

$\begin{array}{lllllllllllllllllllllll}220 & 210 & 200 & 190 & 180 & 170 & 160 & 150 & 140 & 130 & 120 & 110 & 100 & 90 & 80 & 70 & 60 & 50 & 40 & 30 & 20 & 10 & 0\end{array}$ 


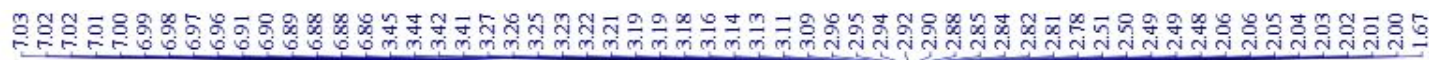
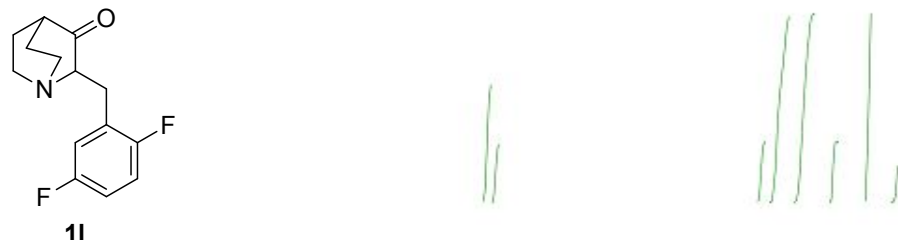

${ }^{1} \mathrm{H} \mathrm{NMR}\left(400 \mathrm{MHz}, \mathrm{CDCl}_{3}\right)$

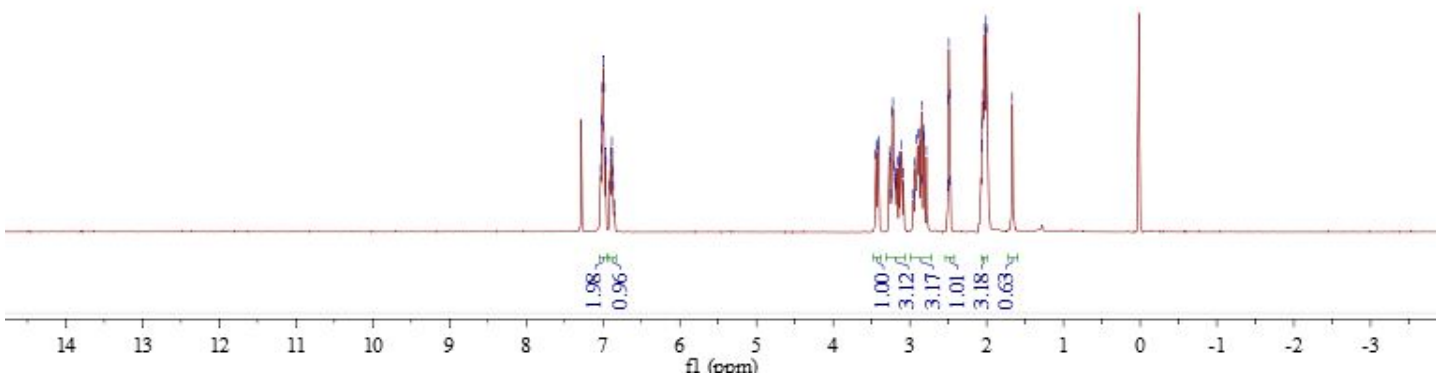

จุ่
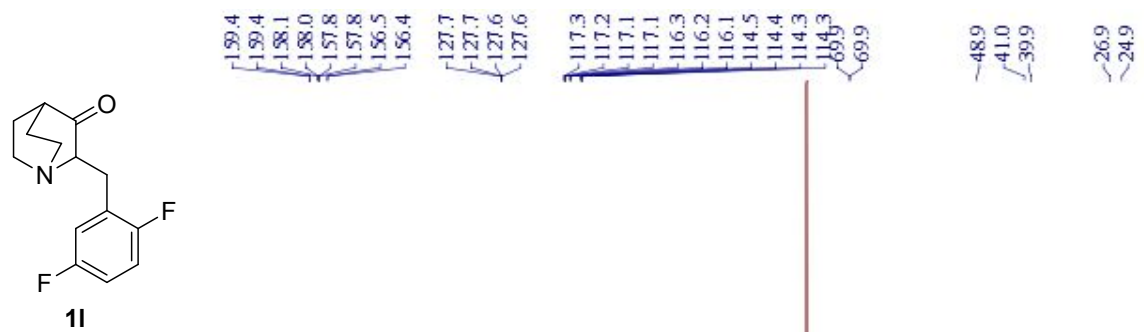

${ }^{13} \mathrm{C}$ NMR $\left(151 \mathrm{MHz}, \mathrm{CDCl}_{3}\right)$

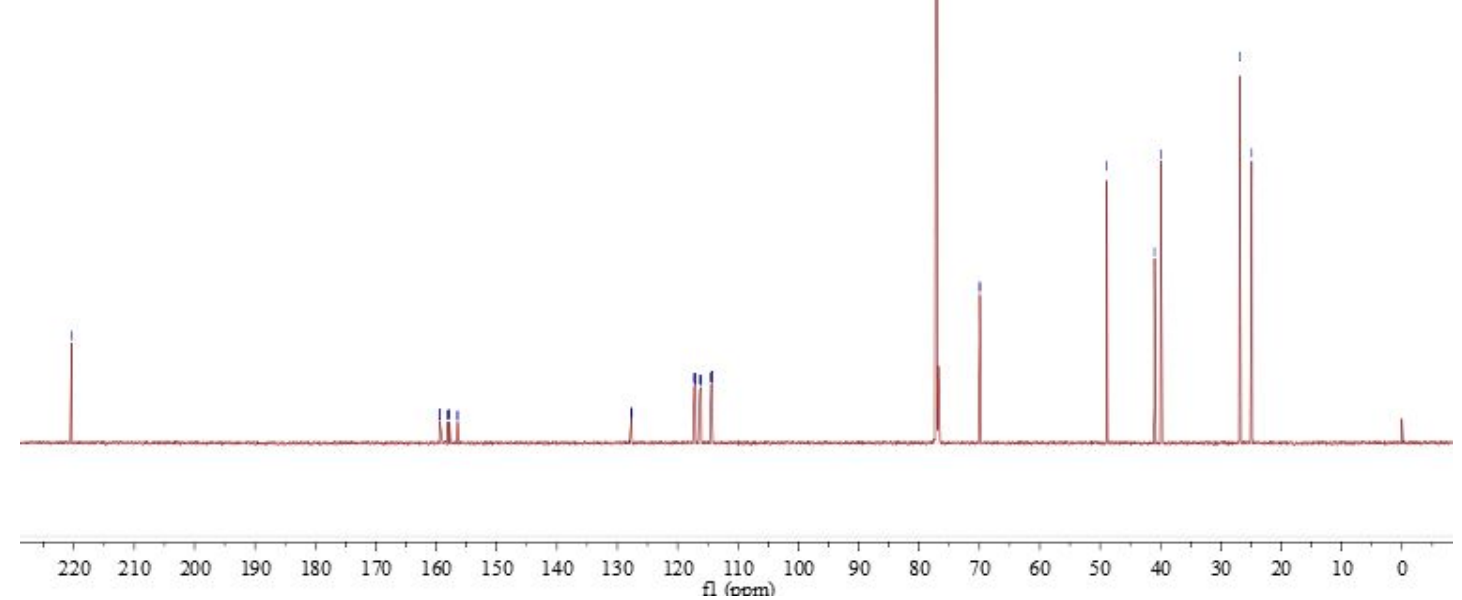




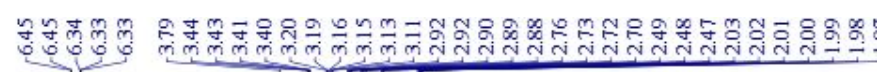
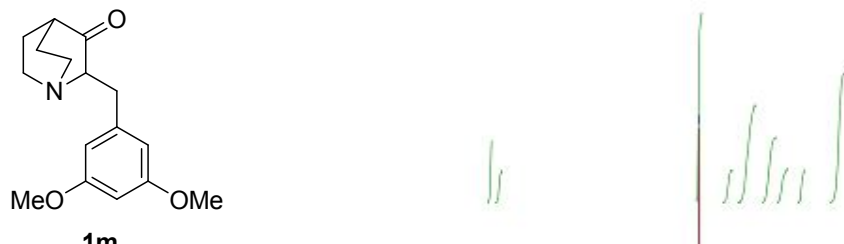

${ }^{1} \mathrm{H}$ NMR $\left(400 \mathrm{MHz}, \mathrm{CDCl}_{3}\right)$

Maid

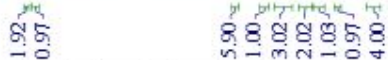

$14 \quad \begin{array}{llllllllllllllllll}1 & 1 & 12 & 11 & 10 & 9 & 8 & 7 & 6 & 5 & 4 & 3 & 2 & 1 & 0 & -1 & -2 & -3\end{array}$

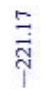

$\frac{1}{8} \quad \frac{1}{8}$

\begin{tabular}{lll}
$\infty$ & $\infty$ \\
0 & $\infty$ \\
\hdashline & $\infty$ \\
\hdashline & $\infty$ & 1
\end{tabular}

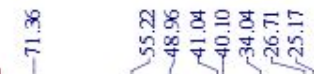

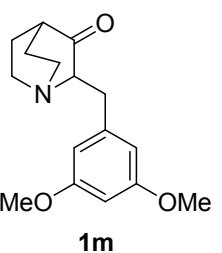

$\left.{ }^{13} \mathrm{C} \mathrm{NMR} \mathrm{(101} \mathrm{MHz,} \mathrm{CDCl}_{3}\right)$

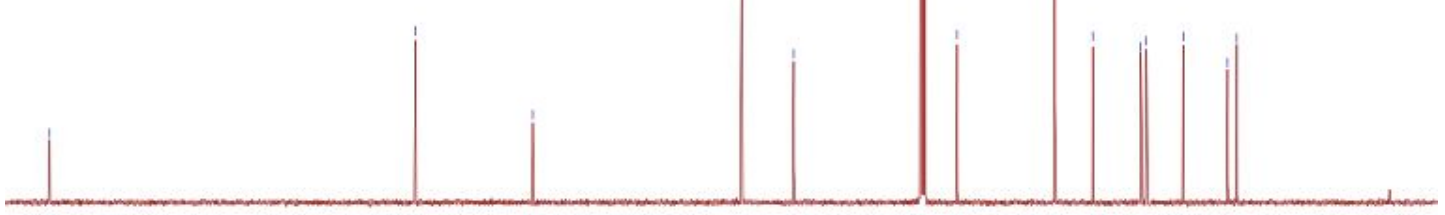

$\begin{array}{lllllllllllllllllllllllllllllllllllllll}220 & 210 & 200 & 190 & 180 & 170 & 160 & 150 & 140 & 130 & 120 & 110 & 100 & 90 & 80 & 70 & 60 & 50 & 40 & 30 & 20 & 10 & 0\end{array}$ 


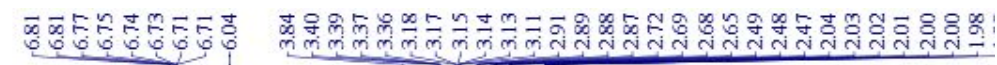
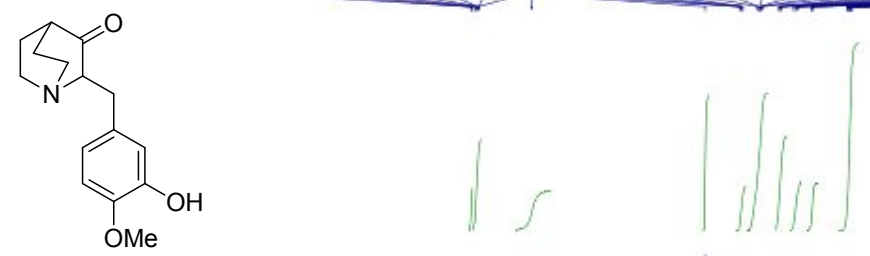

${ }^{1} \mathrm{H} \mathrm{NMR}\left(400 \mathrm{MHz}, \mathrm{CDCl}_{3}\right)$
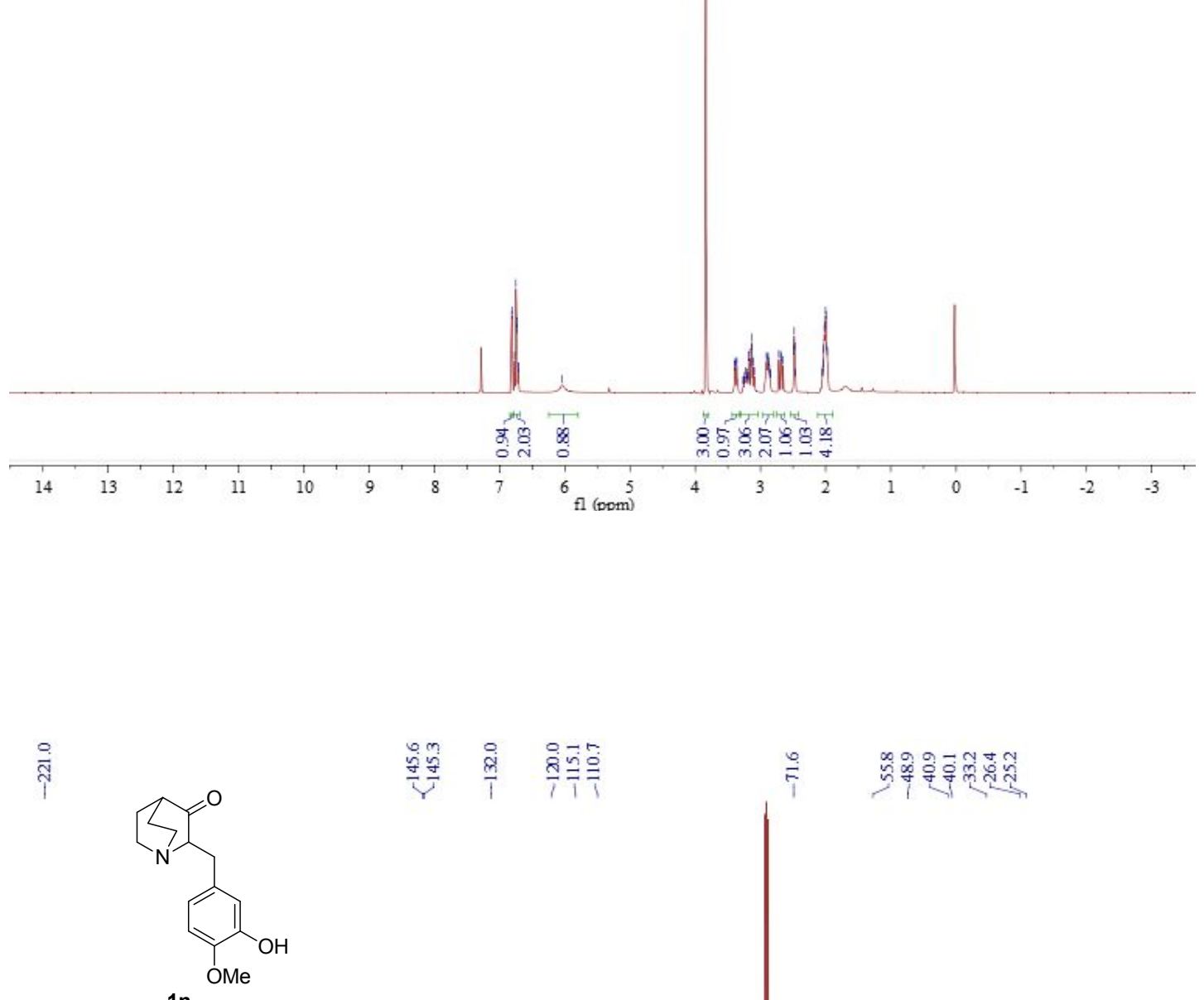

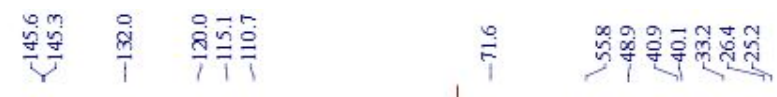

${ }^{13} \mathrm{C} \mathrm{NMR}\left(101 \mathrm{MHz}, \mathrm{CDCl}_{3}\right)$

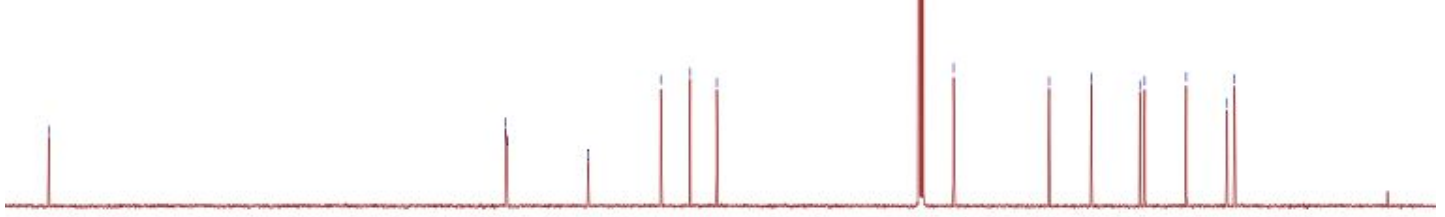

$\begin{array}{lllllllllllllllllllllll}220 & 210 & 200 & 190 & 180 & 170 & 160 & 150 & 140 & 130 & 120 & 110 & 100 & 90 & 80 & 70 & 60 & 50 & 40 & 30 & 20 & 10 & 0\end{array}$ 


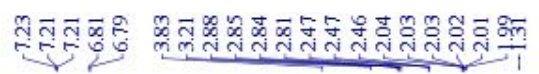
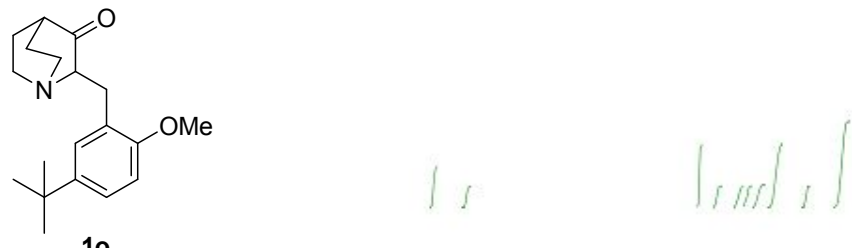

${ }^{1} \mathrm{H}$ NMR (400 MHz, $\mathrm{CDCl}_{3}$ )

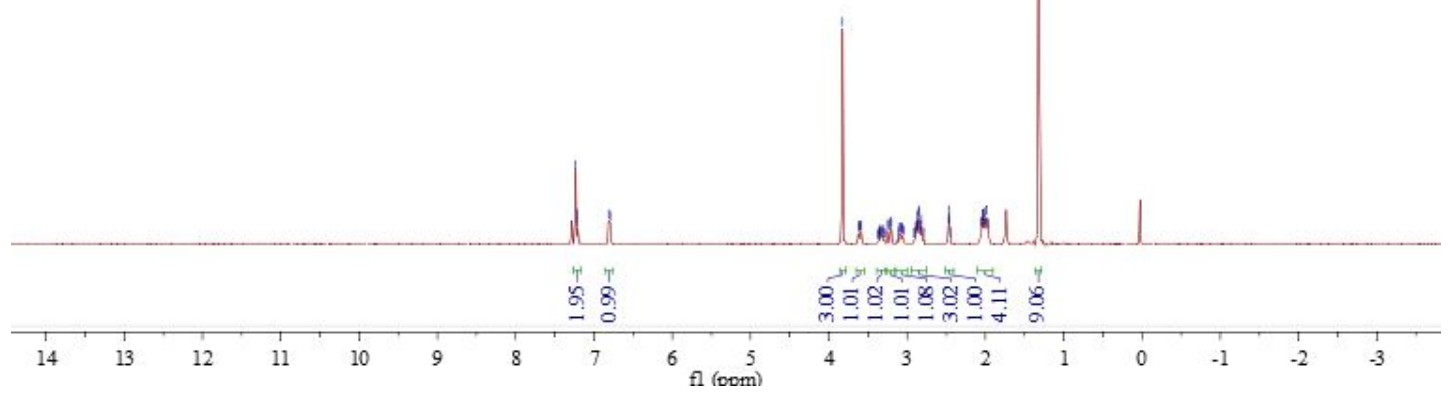

$\stackrel{\infty}{\vec{ก}}$
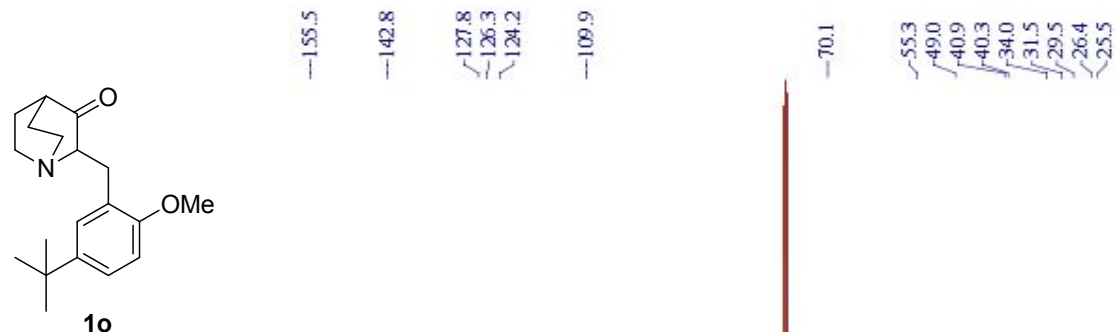

i

${ }^{13} \mathrm{C}$ NMR $\left(101 \mathrm{MHz}, \mathrm{CDCl}_{3}\right)$

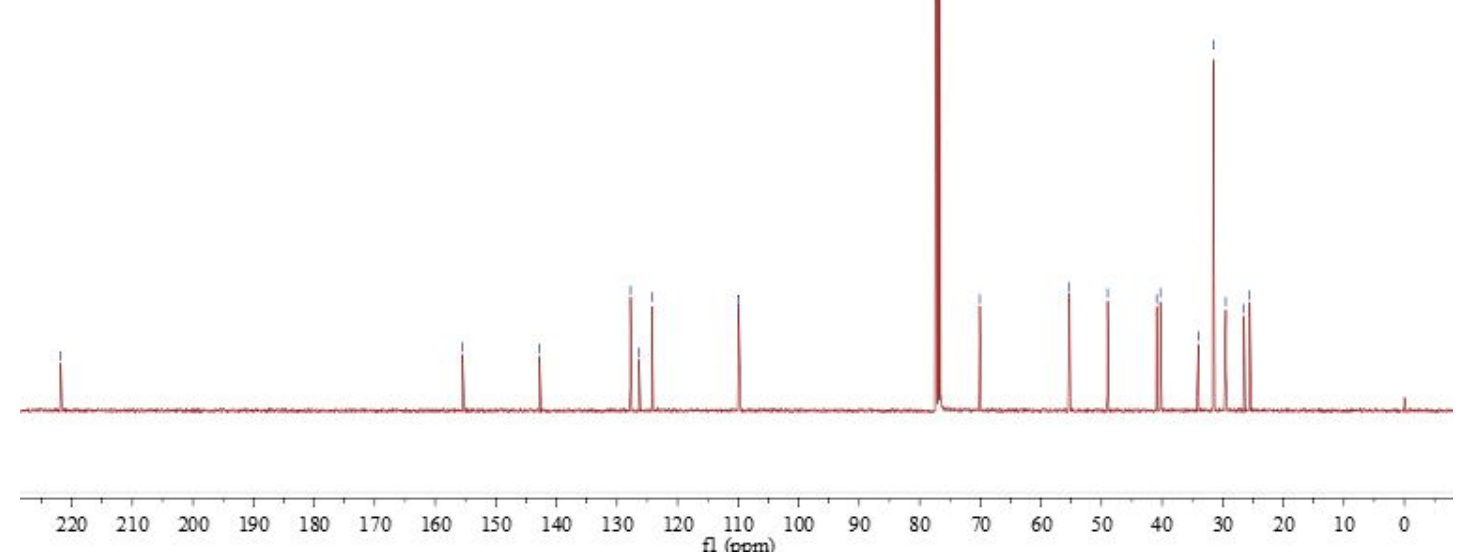




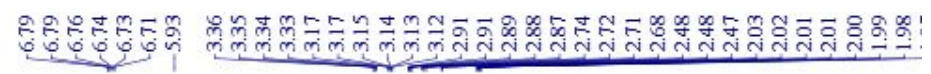
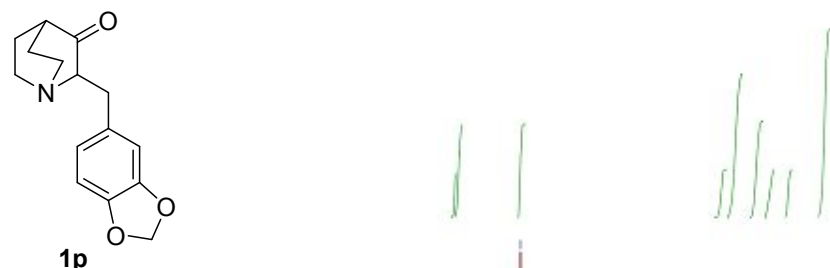

${ }^{1} \mathrm{H}$ NMR $\left(400 \mathrm{MHz}, \mathrm{CDCl}_{3}\right)$

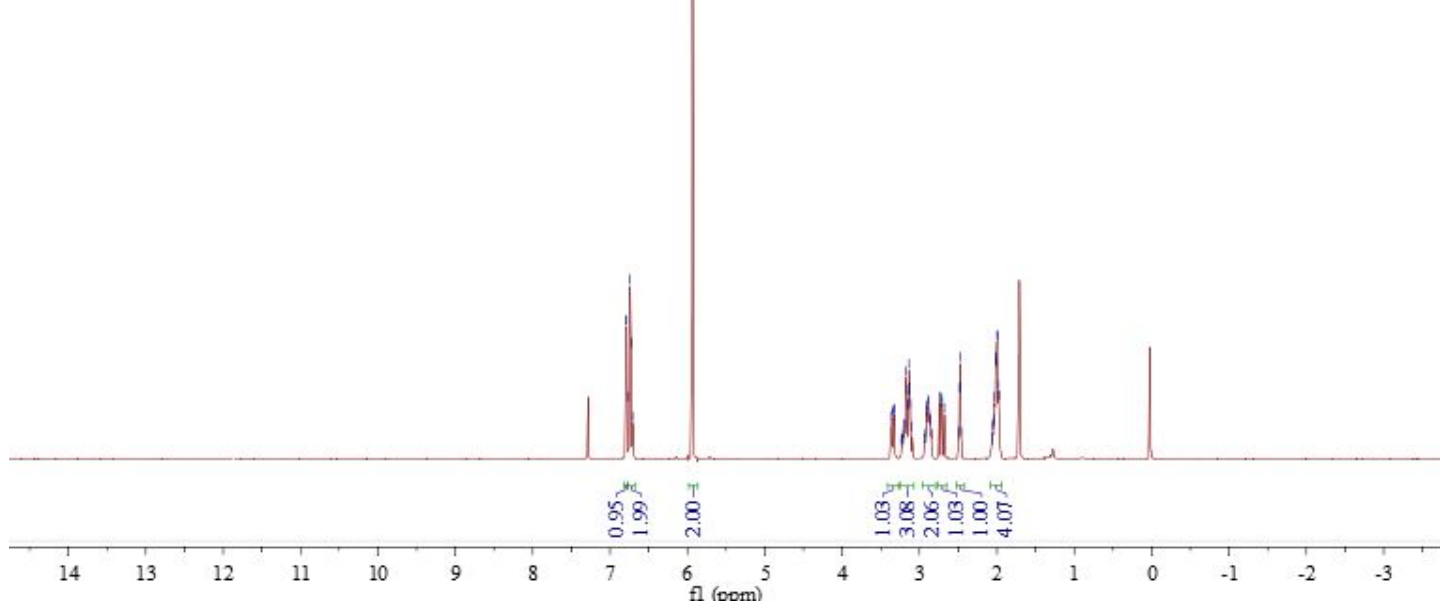

$\overline{\overline{\mathrm{A}}}$

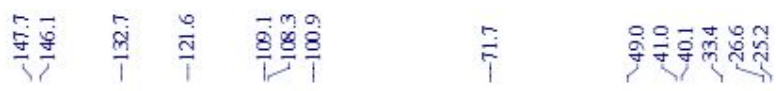

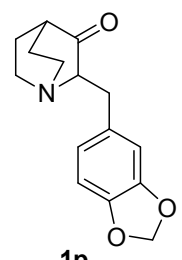

${ }^{13} \mathrm{C}$ NMR $\left(151 \mathrm{MHz}, \mathrm{CDCl}_{3}\right)$

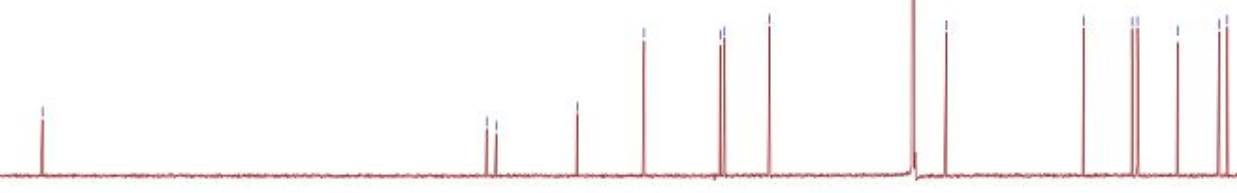

$\begin{array}{llllllllllllllllllllllllllllll}220 & 210 & 200 & 190 & 180 & 170 & 160 & 150 & 140 & 130 & 120 & 110 & 100 & 90 & 80 & 70 & 60 & 50 & 40 & 30 & 20 & 10 & 0\end{array}$ 


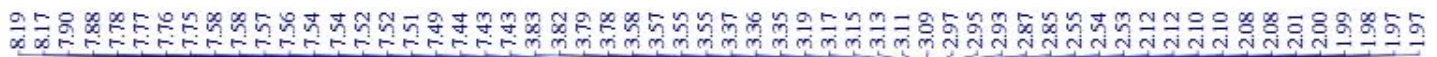
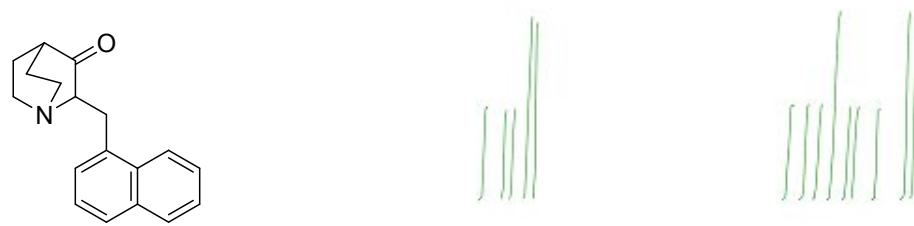

1q

${ }^{1} \mathrm{H}$ NMR (400 MHz, $\mathrm{CDCl}_{3}$ )

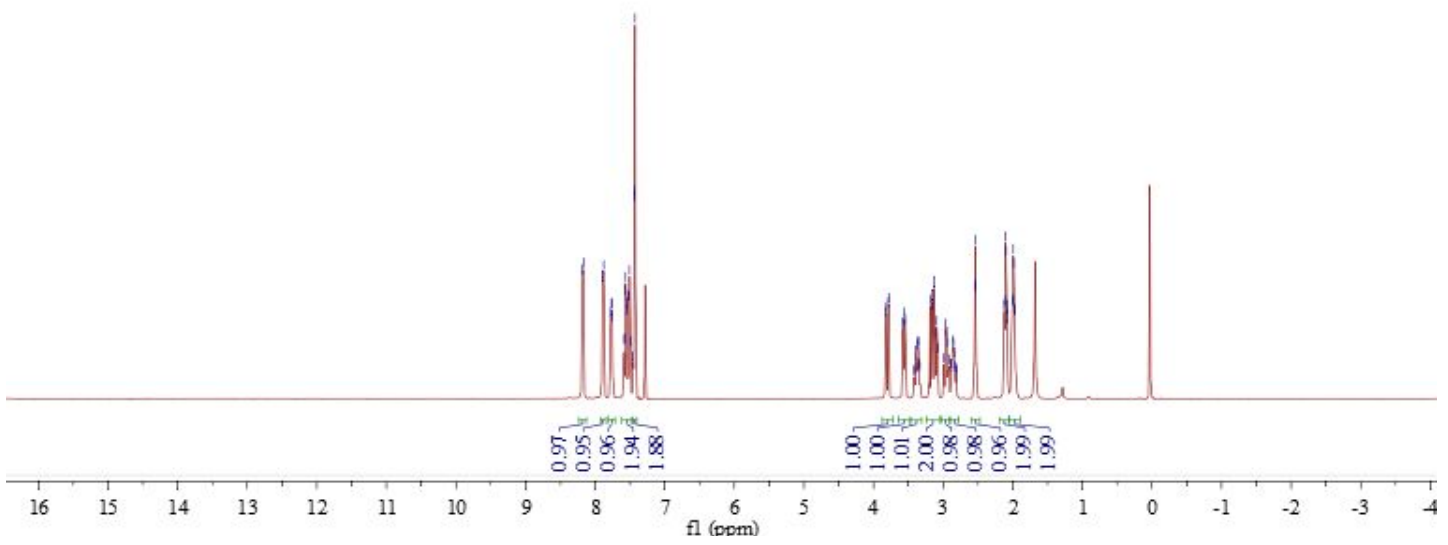

$\stackrel{m}{\uparrow}$

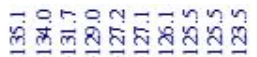

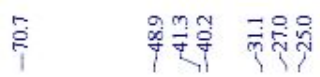

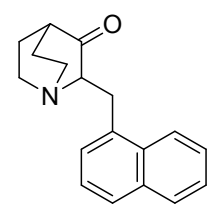

19

${ }^{13} \mathrm{C}$ NMR (101 MHz, $\mathrm{CDCl}_{3}$ )

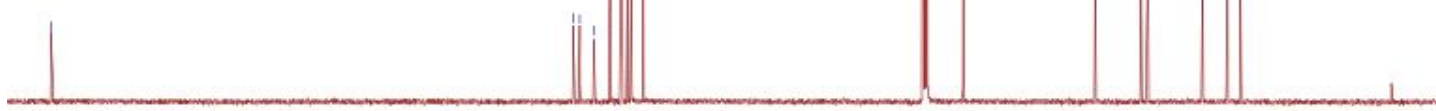

$\begin{array}{lllllllllllllllllllllll}220 & 210 & 200 & 190 & 180 & 170 & 160 & 150 & 140 & 130 & 120 & 110 & 100 & 90 & 80 & 70 & 60 & 50 & 40 & 30 & 20 & 10 & 0\end{array}$ 


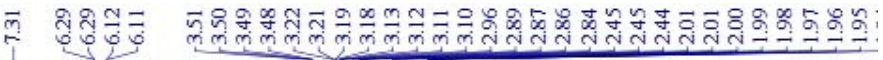
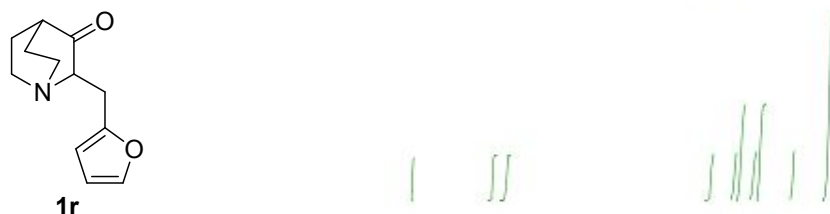

${ }^{1} \mathrm{H}$ NMR $\left(600 \mathrm{MHz}, \mathrm{CDCl}_{3}\right)$

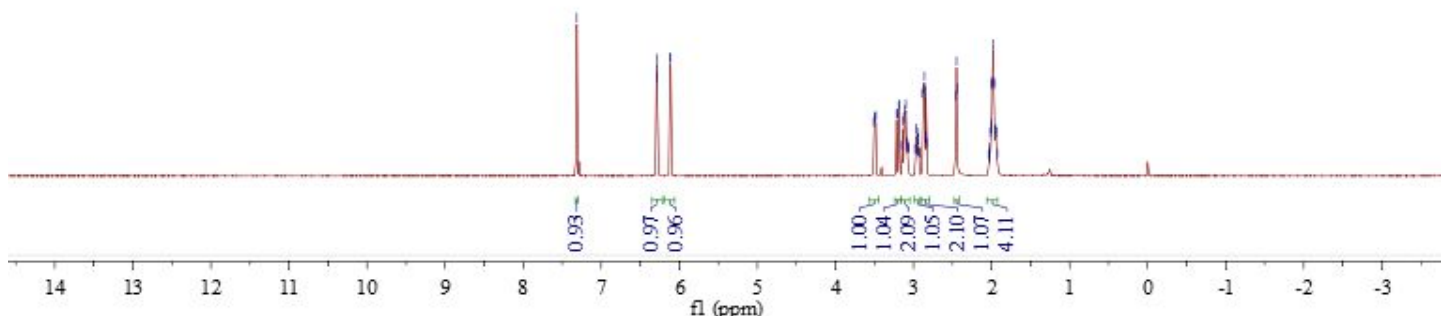

ชิ่

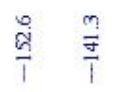

$\stackrel{+口}{\circ}$

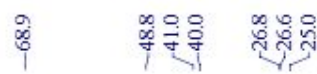

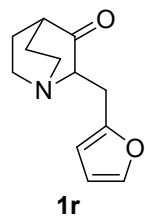

${ }^{13} \mathrm{C}$ NMR $\left(151 \mathrm{MHz}, \mathrm{CDCl}_{3}\right.$ )

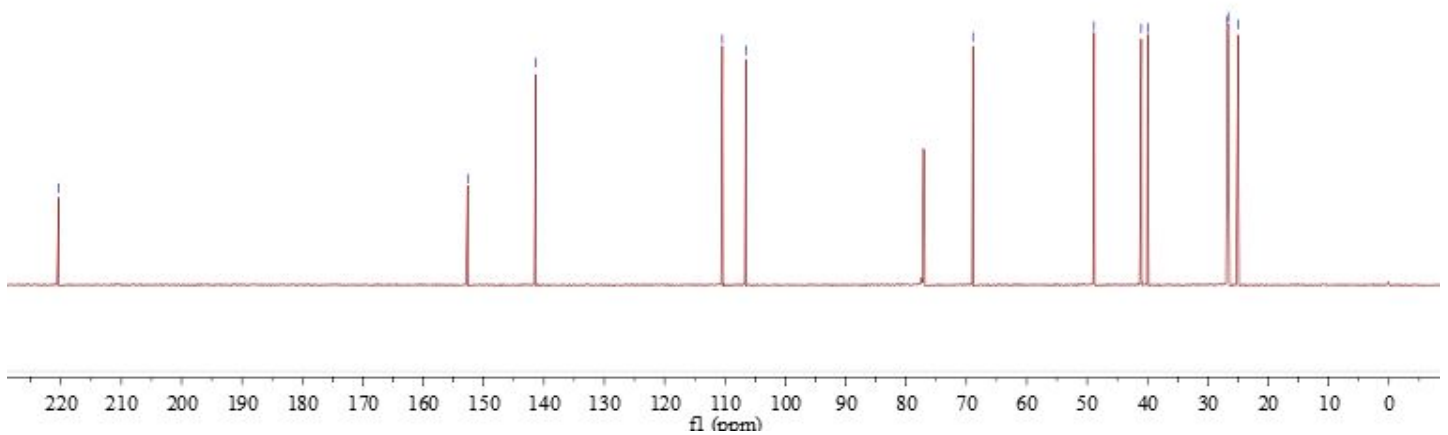




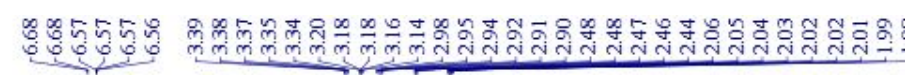
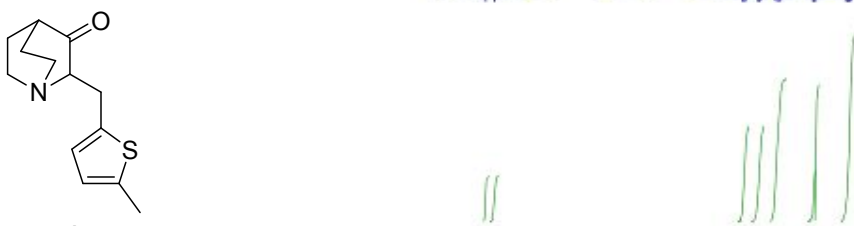

${ }^{1} \mathrm{H} \mathrm{NMR}\left(400 \mathrm{MHz}, \mathrm{CDCl}_{3}\right)$

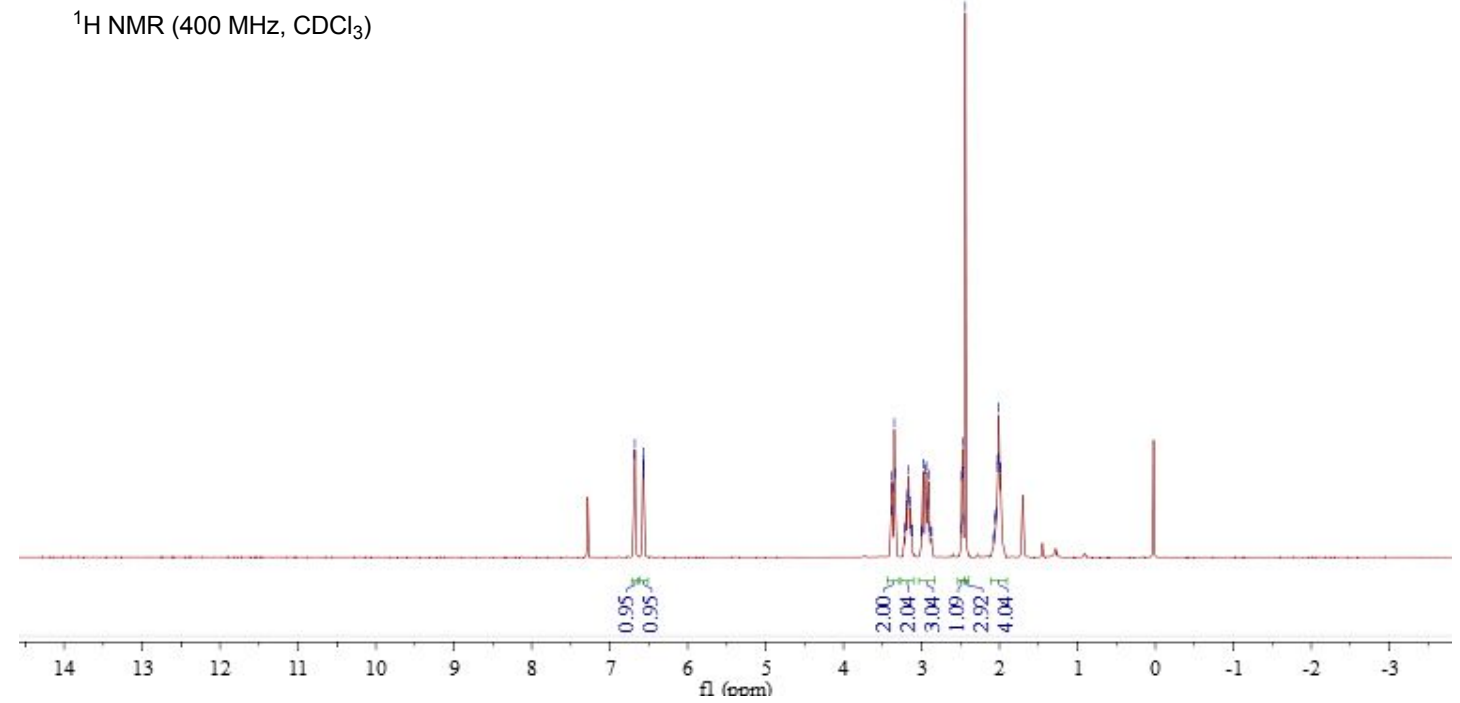

ஷิ

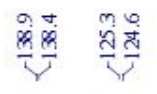

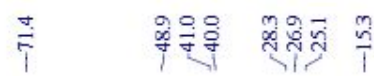

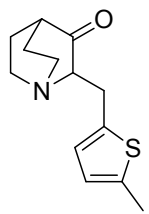

遂遂

1s

${ }^{13} \mathrm{C}$ NMR $\left(151 \mathrm{MHz}, \mathrm{CDCl}_{3}\right)$

$\begin{array}{llllllllllllllllllllllllllllllllll}220 & 210 & 200 & 190 & 180 & 170 & 160 & 150 & 140 & 130 & 120 & 110 & 100 & 90 & 80 & 70 & 60 & 50 & 40 & 30 & 20 & 10 & 0\end{array}$ 


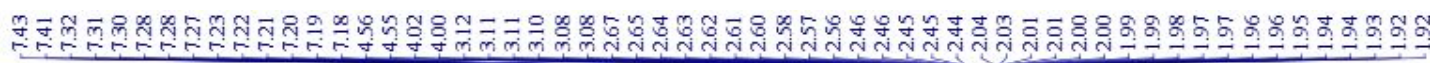
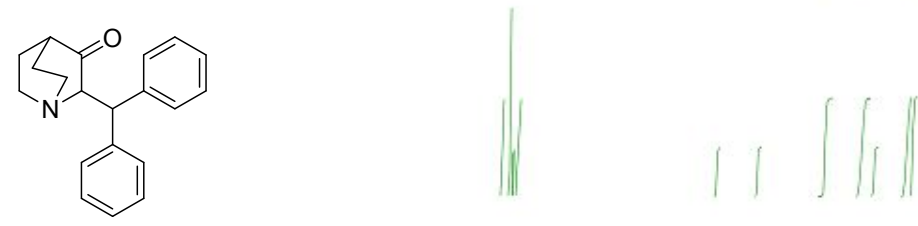

${ }^{1} \mathrm{H}$ NMR $\left(600 \mathrm{MHz}, \mathrm{CDCl}_{3}\right)$

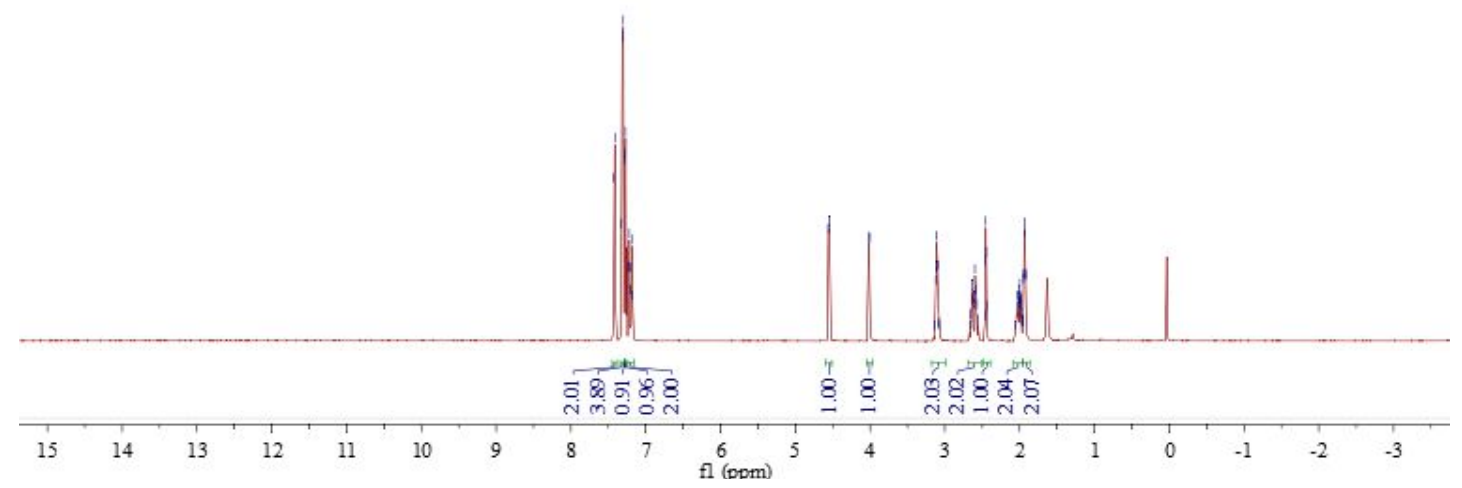

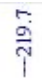

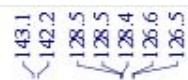

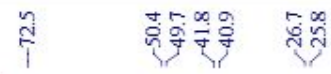

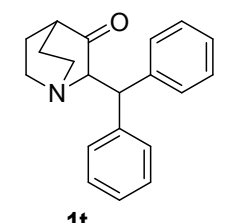

1t

${ }^{13} \mathrm{C}$ NMR $\left(101 \mathrm{MHz}, \mathrm{CDCl}_{3}\right)$

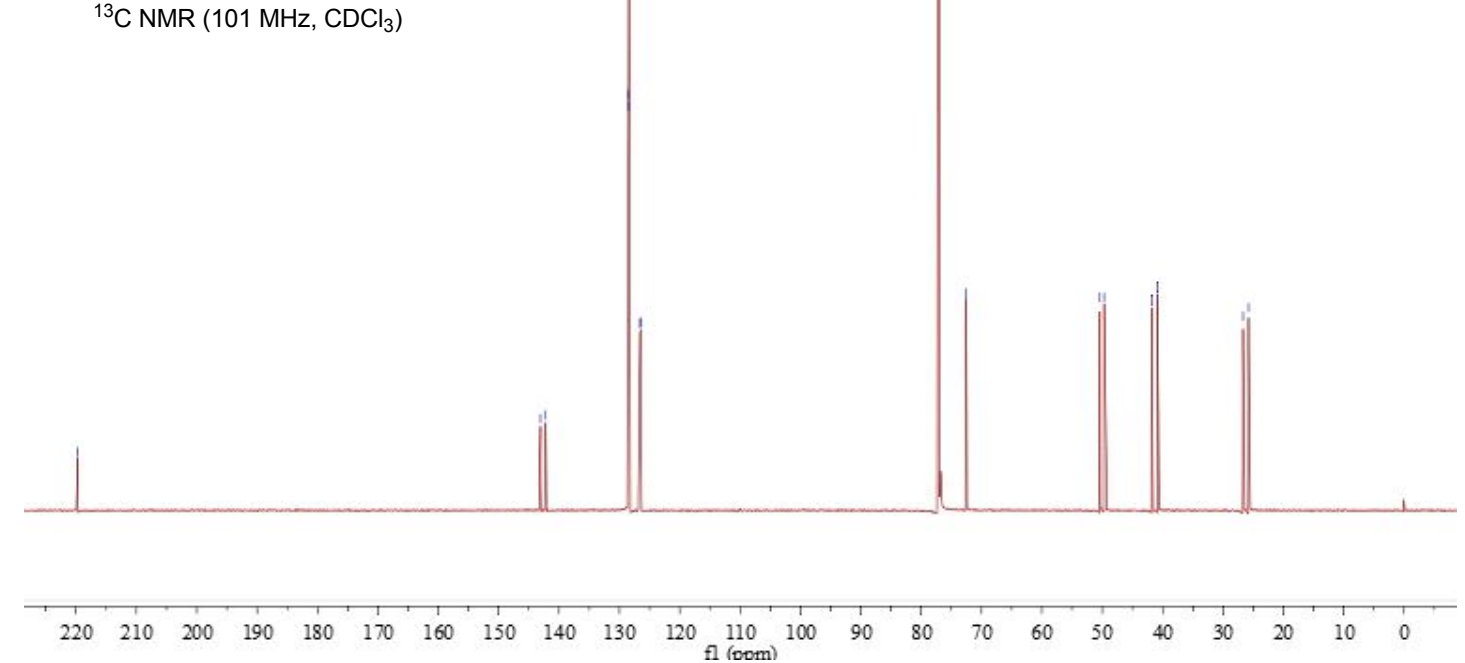




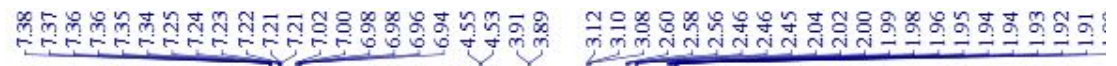
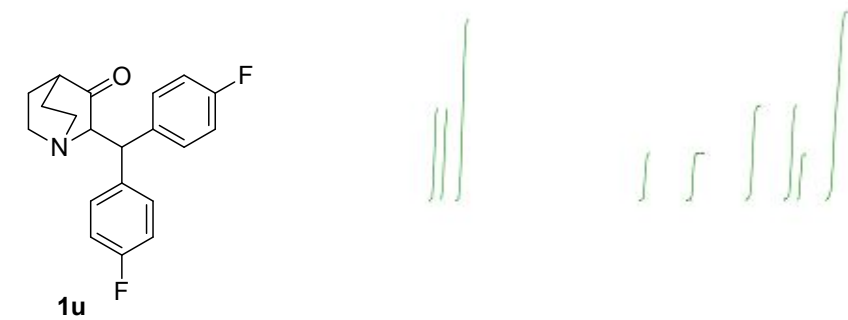

${ }^{1} \mathrm{H} \mathrm{NMR}\left(400 \mathrm{MHz}, \mathrm{CDCl}_{3}\right)$

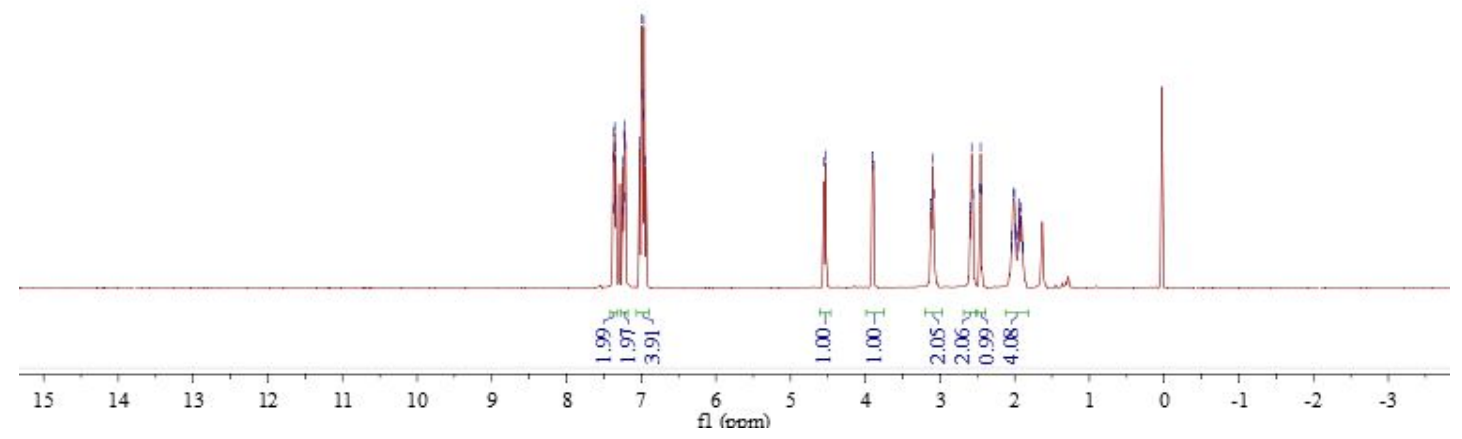

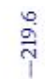

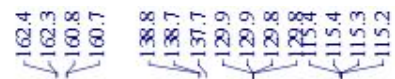

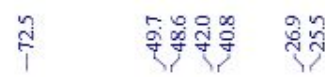

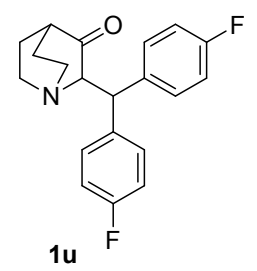

${ }^{13} \mathrm{C}$ NMR $\left(151 \mathrm{MHz}, \mathrm{CDCl}_{3}\right)$

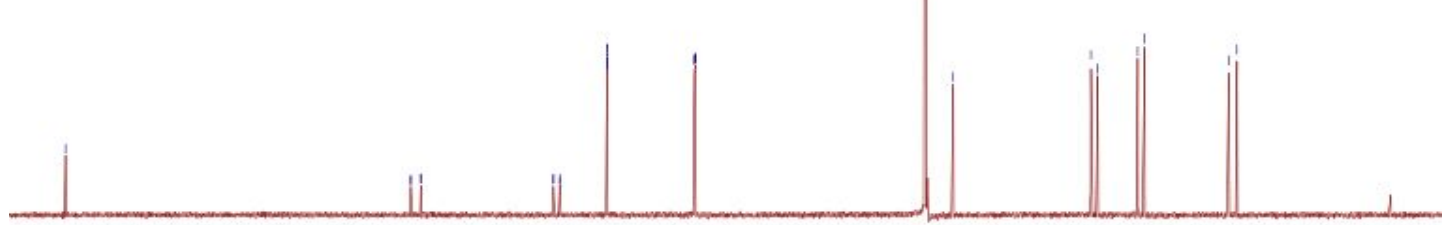

$\begin{array}{lllllllllllllllllllllll}220 & 210 & 200 & 190 & 180 & 170 & 160 & 150 & 140 & 130 & 120 & 110 & 100 & 90 & 80 & 70 & 60 & 50 & 40 & 30 & 20 & 10 & 0\end{array}$ 


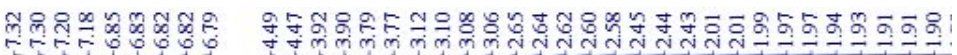
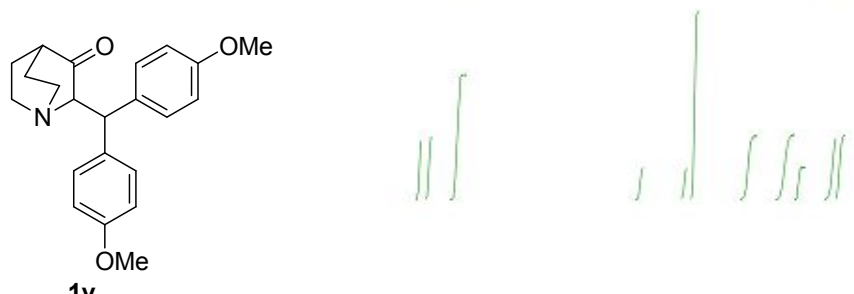

${ }^{1} \mathrm{H}$ NMR $\left(400 \mathrm{MHz}, \mathrm{CDCl}_{3}\right)$

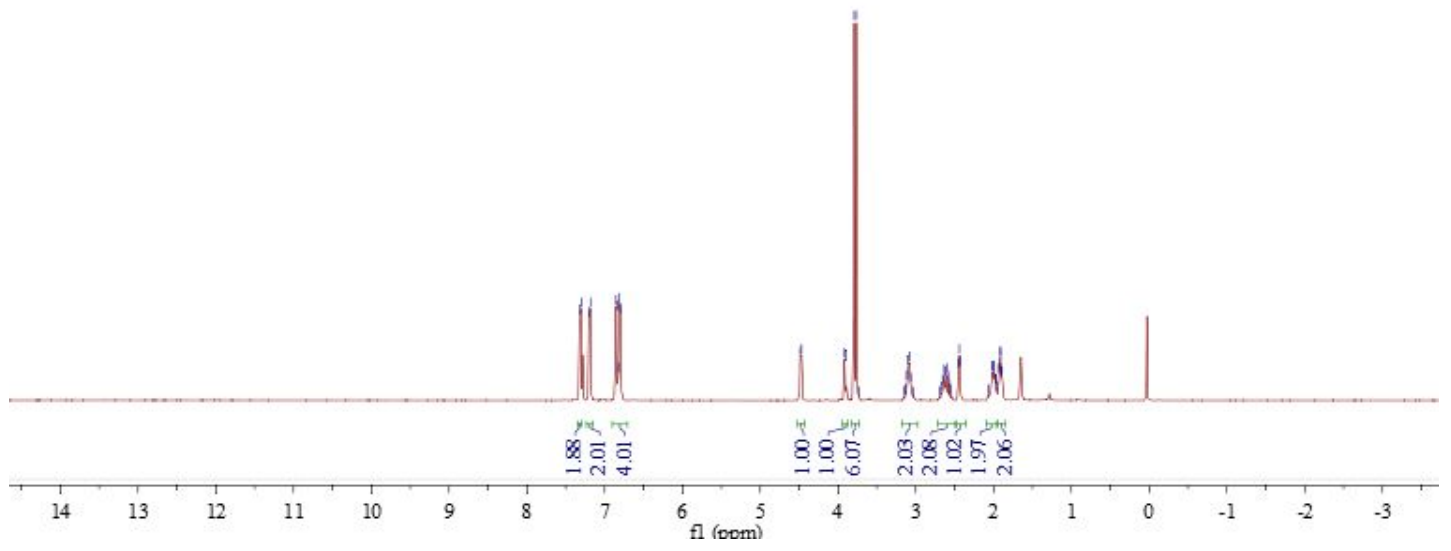

$\stackrel{\circ}{\frac{9}{1}}$

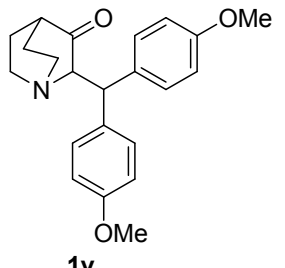

1v

${ }^{13} \mathrm{C} \mathrm{NMR}\left(101 \mathrm{MHz}, \mathrm{CDCl}_{3}\right)$

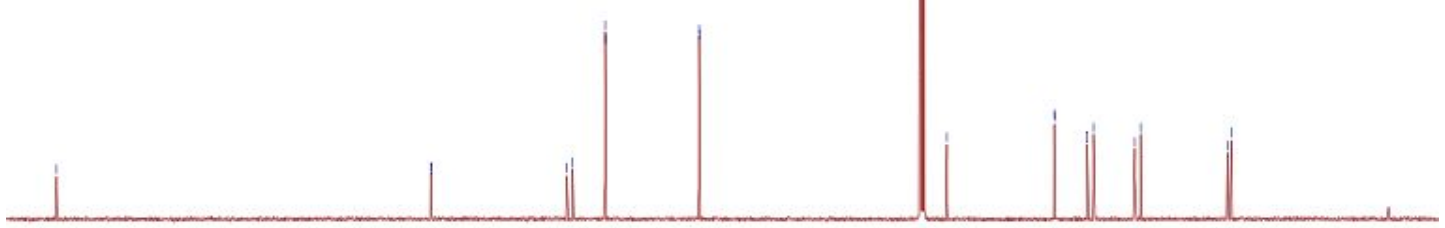

$\begin{array}{lllllllllllllllllllllll}220 & 210 & 200 & 190 & 180 & 170 & 160 & 150 & 140 & 130 & 120 & 110 & 100 & 90 & 80 & 70 & 60 & 50 & 40 & 30 & 20 & 10 & 0\end{array}$ 


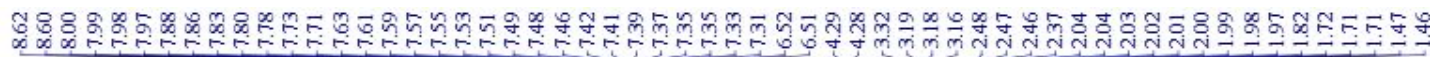<smiles>O=C1C2CCN(C2)C1C(c1cccc2ccccc12)c1cccc2ccccc12</smiles>

1w

${ }^{1} \mathrm{H} \mathrm{NMR}\left(400 \mathrm{MHz}, \mathrm{CDCl}_{3}\right)$
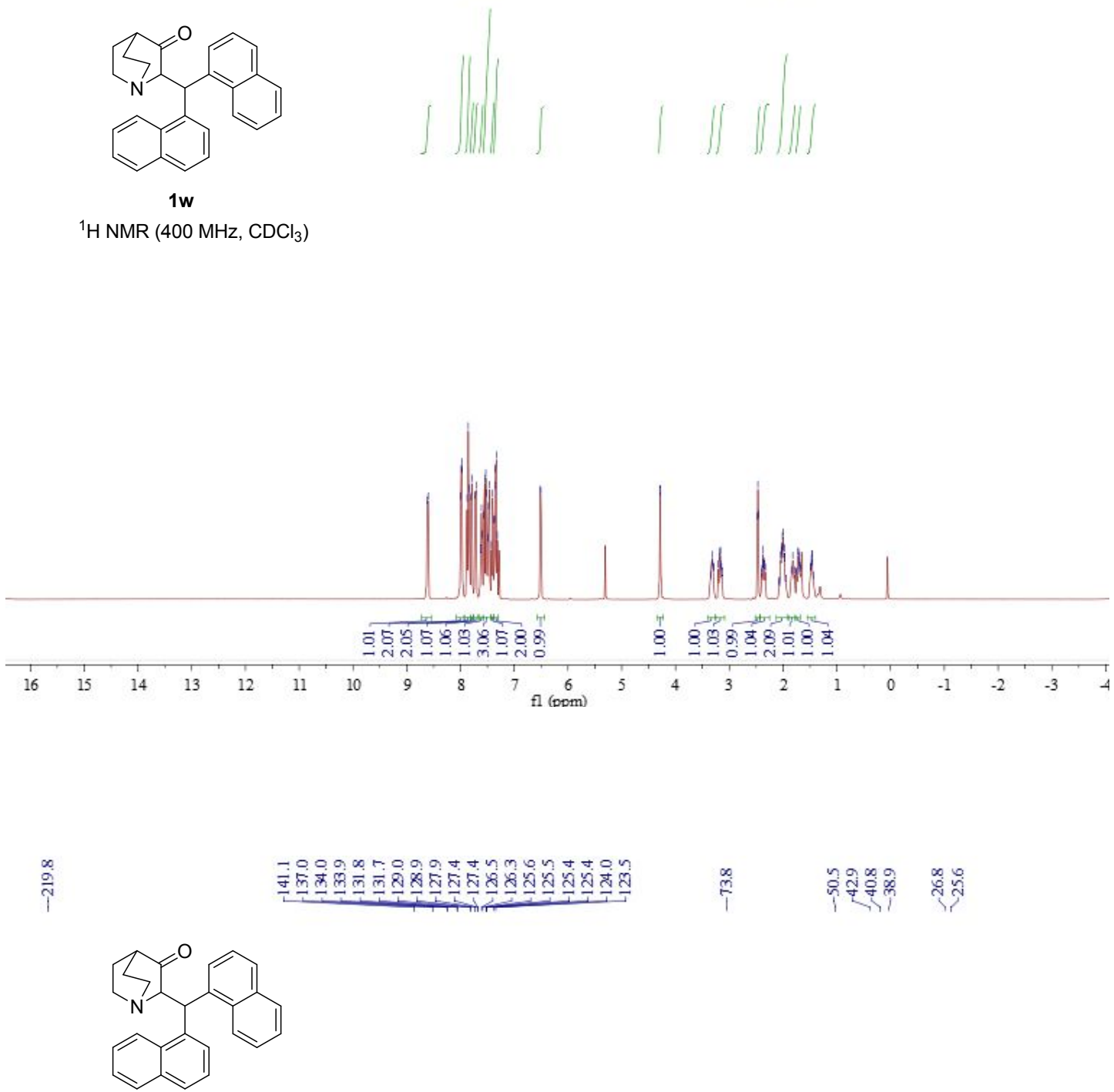

$1 w$

${ }^{13} \mathrm{C} \mathrm{NMR}\left(151 \mathrm{MHz}, \mathrm{CDCl}_{3}\right)$

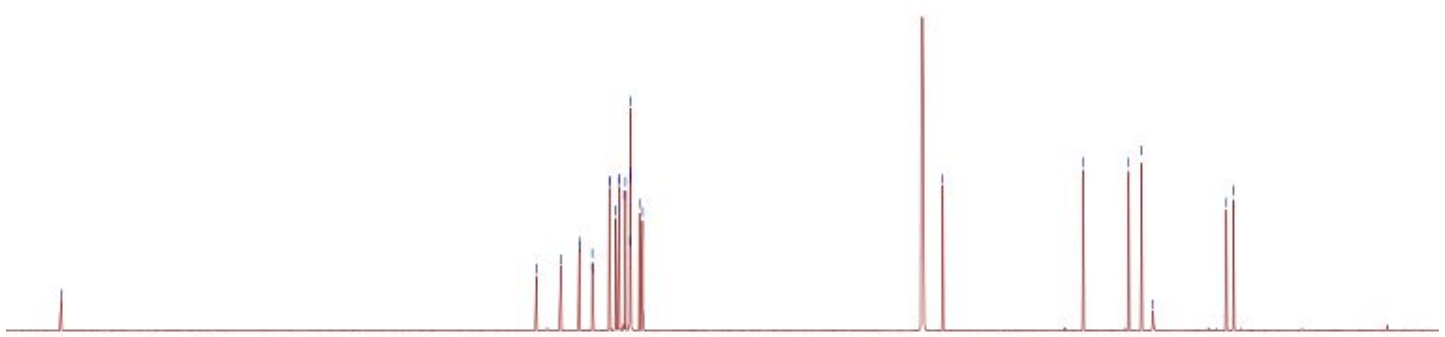

$\begin{array}{lllllllllllllllllllllllllllllllllll}220 & 210 & 200 & 190 & 180 & 170 & 160 & 150 & 140 & 130 & 120 & 110 & 100 & 90 & 80 & 70 & 60 & 50 & 40 & 30 & 20 & 10 & 0\end{array}$ 
8. NMR spectra for $2 a-w$

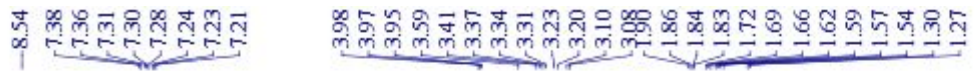
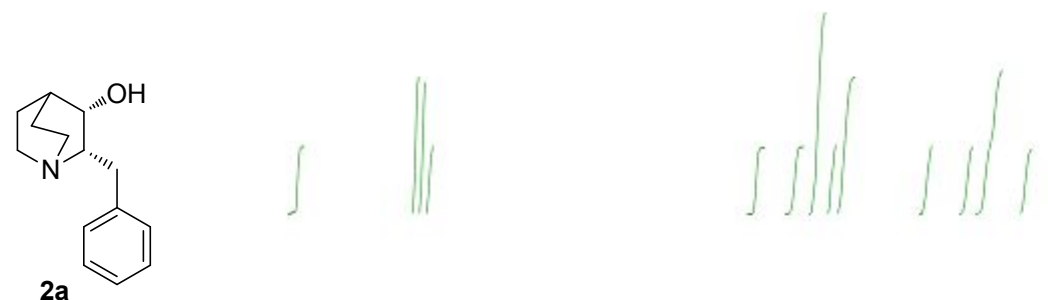

${ }^{1} \mathrm{H} \mathrm{NMR}\left(400 \mathrm{MHz}, \mathrm{CDCl}_{3}\right)$

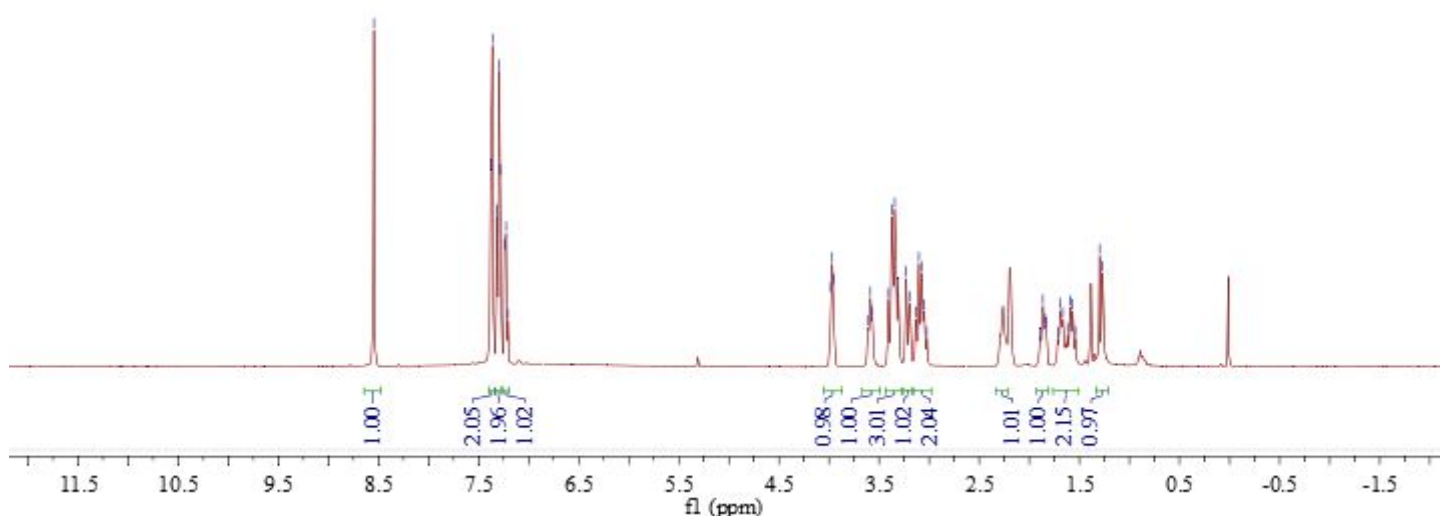

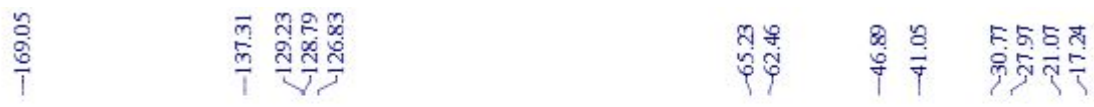

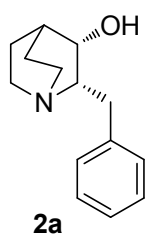

${ }^{13} \mathrm{C} \mathrm{NMR}\left(151 \mathrm{MHz}, \mathrm{CDCl}_{3}\right)$

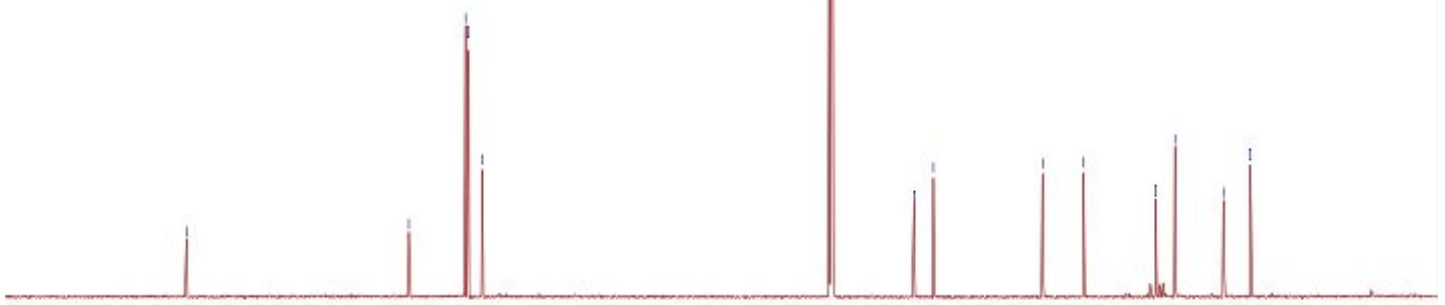

190

$\begin{array}{llllllllll}180 & 170 & 160 & 150 & 140 & 130 & 120 & 110 & 100 & 90\end{array}$ 

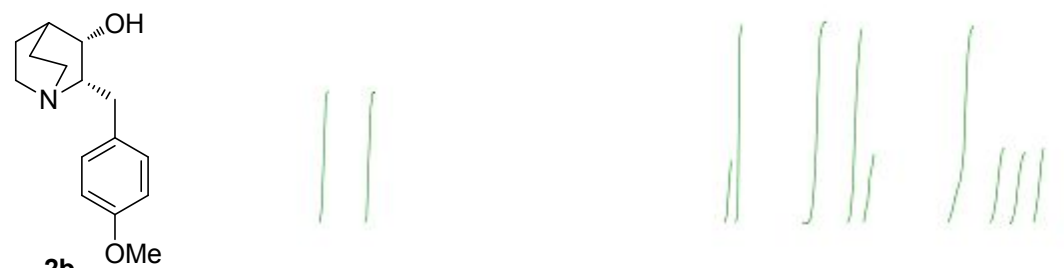

${ }^{1} \mathrm{H}$ NMR $\left(400 \mathrm{MHz}, \mathrm{CDCl}_{3}\right)$
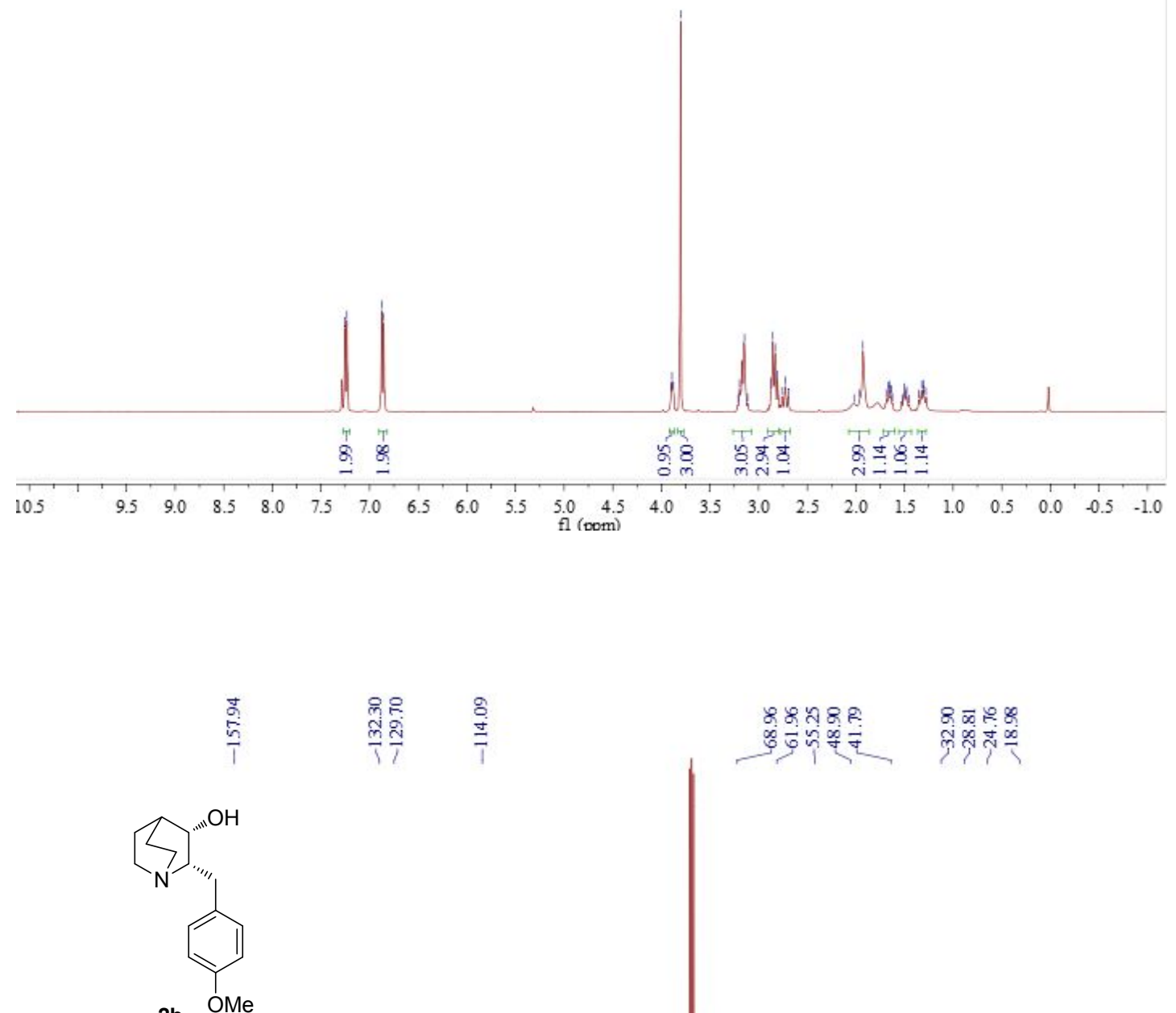

2b

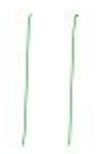

${ }^{13} \mathrm{C} \mathrm{NMR}\left(101 \mathrm{MHz}, \mathrm{CDCl}_{3}\right)$

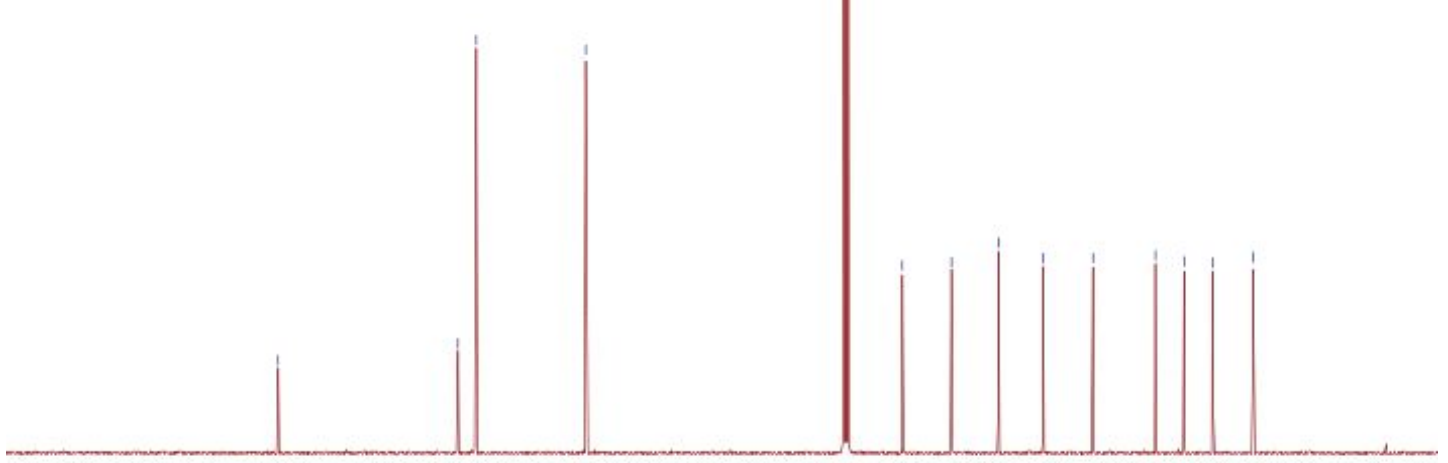

$\begin{array}{llllllllllllllllllll}190 & 180 & 170 & 160 & 150 & 140 & 130 & 120 & 110 & 100 & 90 & 80 & 70 & 60 & 50 & 40 & 30 & 20 & 10 & 0\end{array}$ 


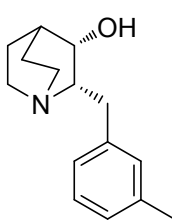

2c

${ }^{1} \mathrm{H} \mathrm{NMR}\left(400 \mathrm{MHz}, \mathrm{CDCl}_{3}\right)$
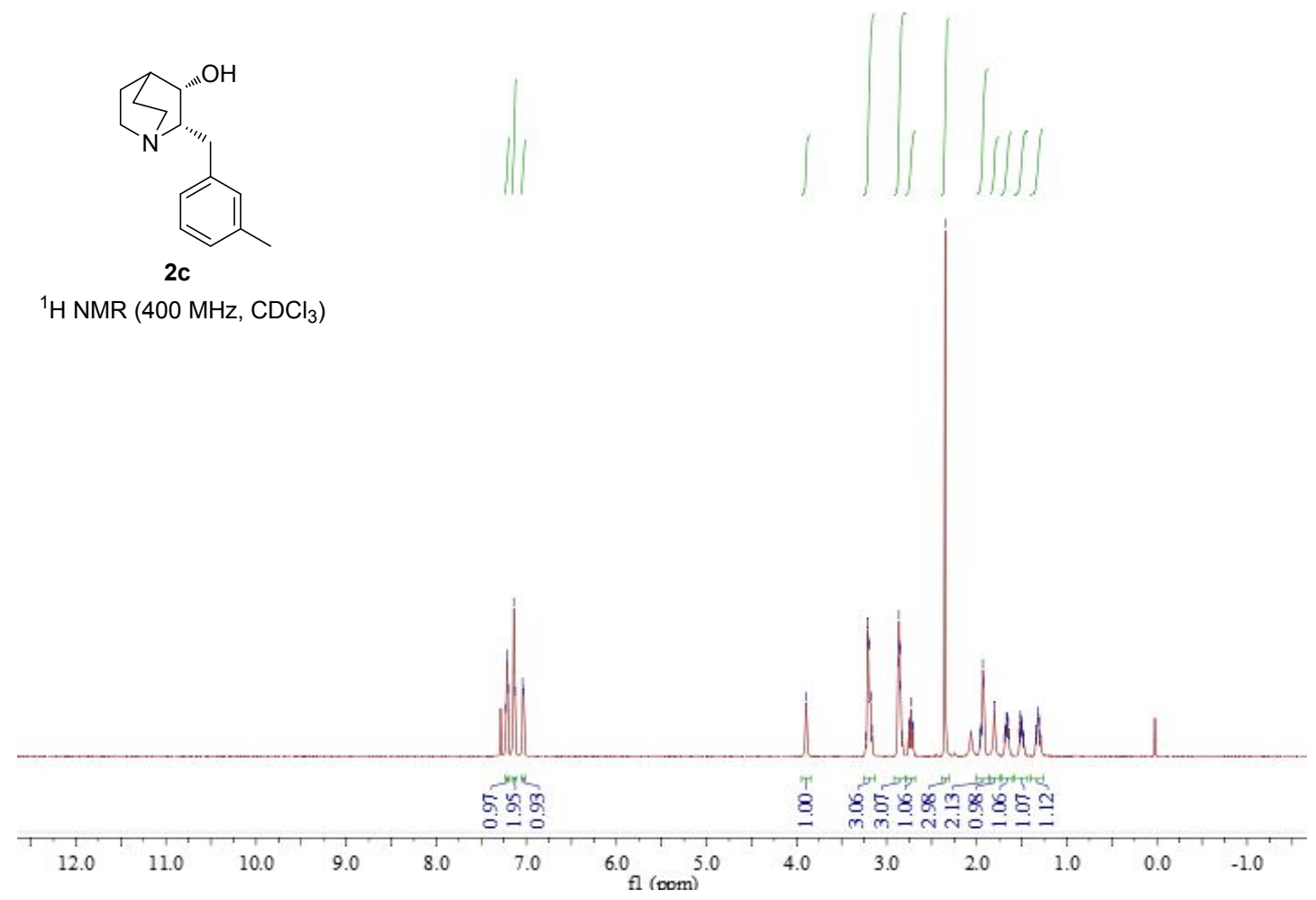

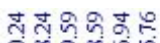

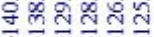

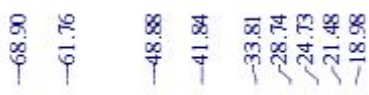

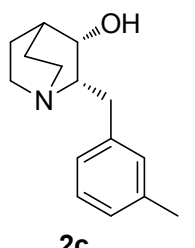

2c

${ }^{13} \mathrm{C} \mathrm{NMR}\left(151 \mathrm{MHz}, \mathrm{CDCl}_{3}\right)$

(2)

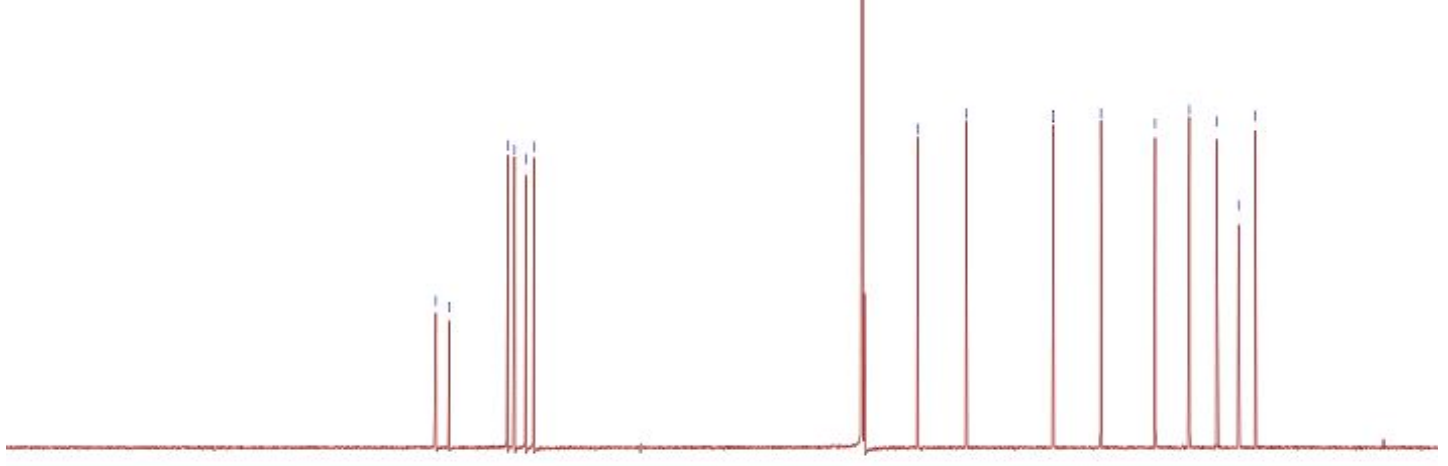

$\begin{array}{lllllllllllllllllllll}200 & 190 & 180 & 170 & 160 & 150 & 140 & 130 & 120 & 110 & 100 & 90 & 80 & 70 & 60 & 50 & 40 & 30 & 20 & 10 & 0\end{array}$ 


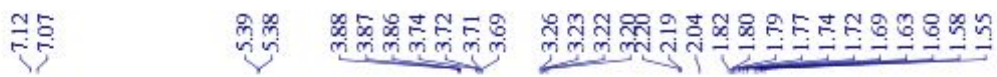

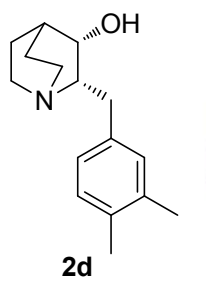

${ }^{1} \mathrm{H}$ NMR $\left(400 \mathrm{MHz}\right.$, DMSO- $\left.d_{6}\right)$

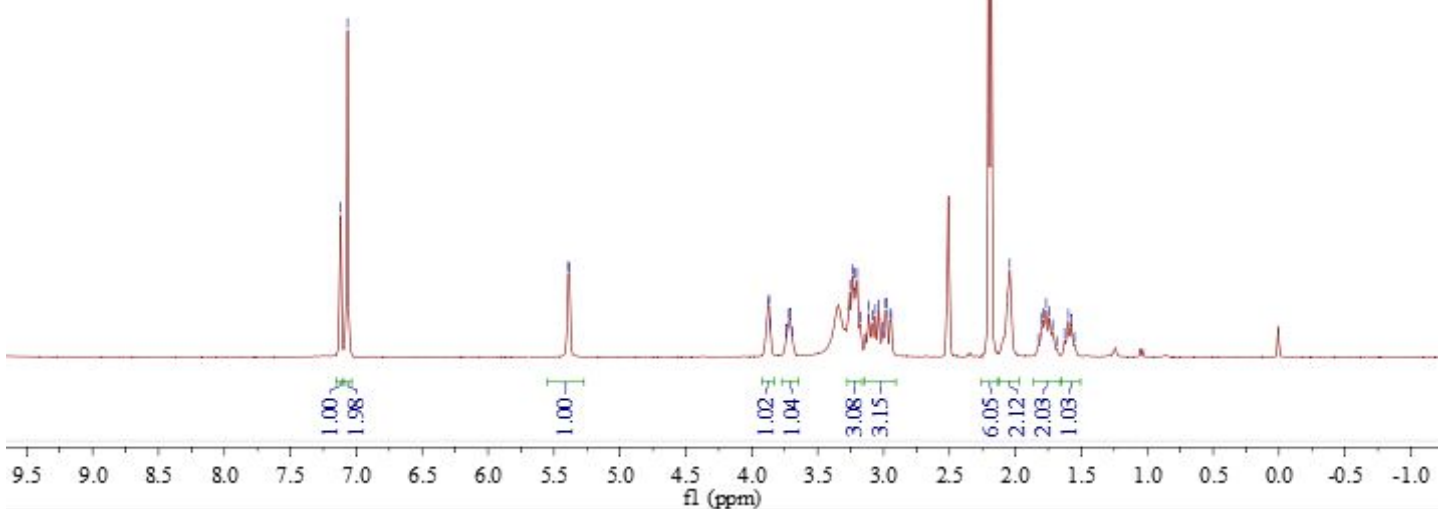

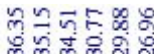

舟

$\sum_{N}^{\prime \prime \prime \prime \prime \prime \prime ~}$

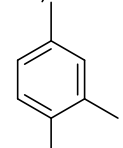

2d

${ }^{13} \mathrm{C}$ NMR $\left(101 \mathrm{MHz}\right.$, DMSO- $\left.d_{6}\right)$

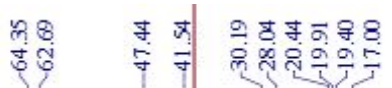

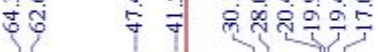

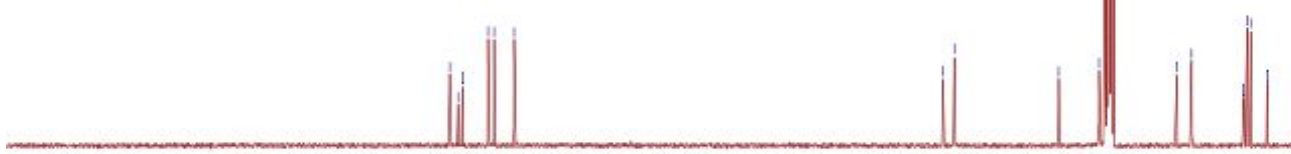

$100 \quad 190$

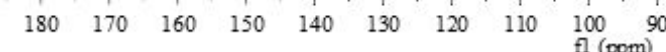




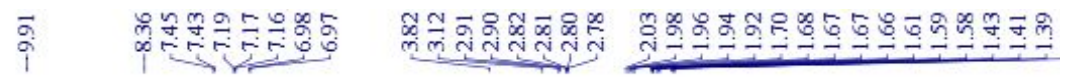
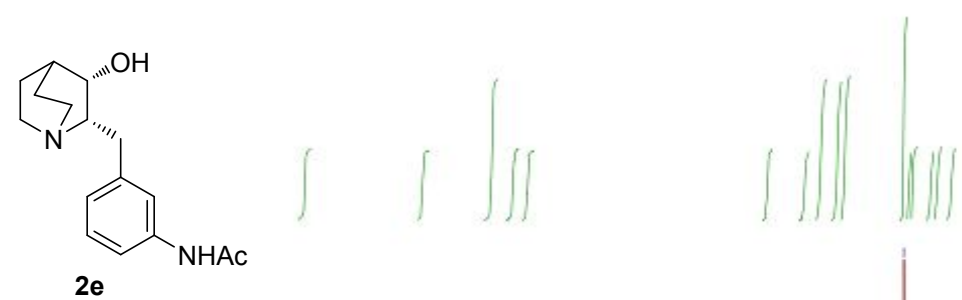

${ }^{1} \mathrm{H}$ NMR $\left(400 \mathrm{MHz}\right.$, DMSO- $\left.d_{6}\right)$

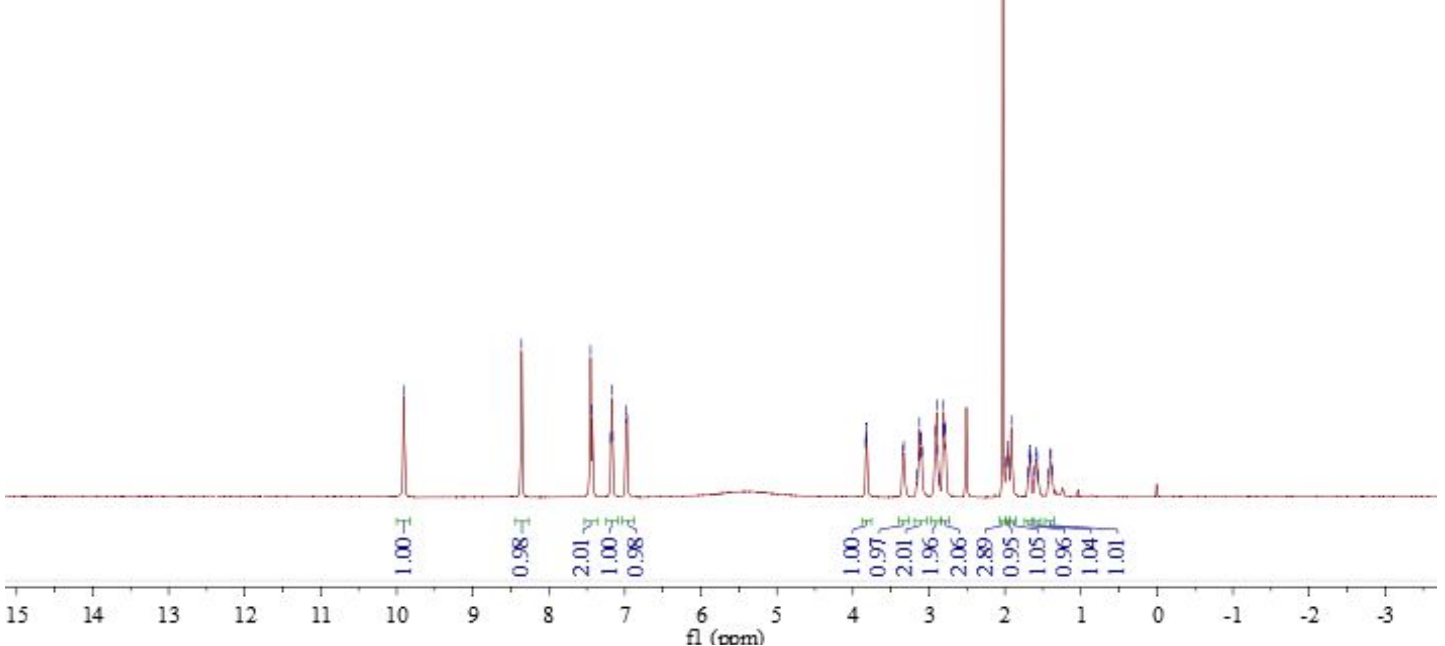

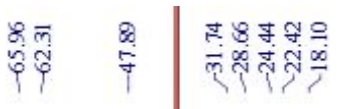

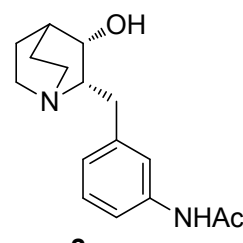

${ }^{13} \mathrm{C}$ NMR $\left(151 \mathrm{MHz}\right.$, DMSO- $\left.d_{6}\right)$

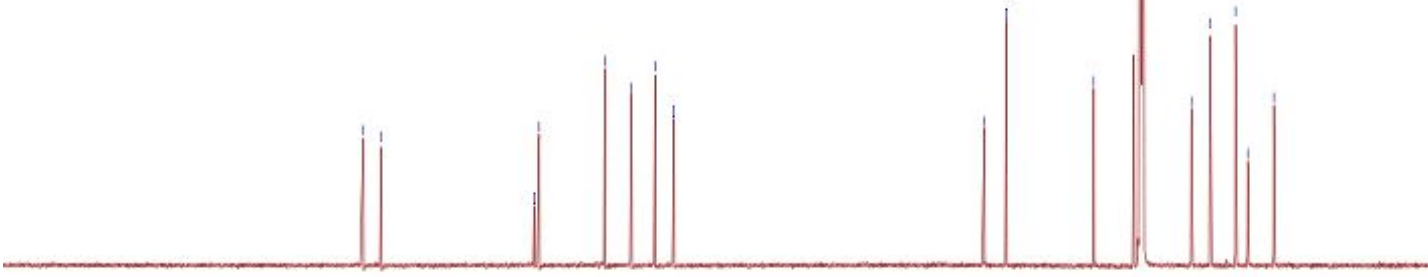

$\begin{array}{lllllllllllllllllllllllllllllllll}220 & 210 & 200 & 190 & 180 & 170 & 160 & 150 & 140 & 130 & 120 & 110 & 100 & 90 & 80 & 70 & 60 & 50 & 40 & 30 & 20 & 10 & 0\end{array}$ 


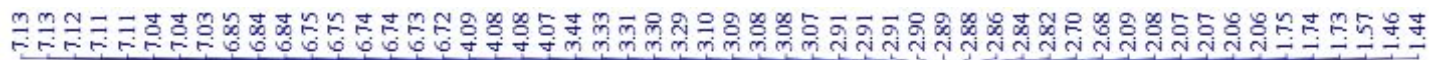

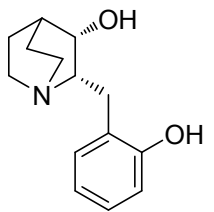

$2 f$

${ }^{1} \mathrm{H} \mathrm{NMR}\left(400 \mathrm{MHz}, \mathrm{CDCl}_{3}\right)$
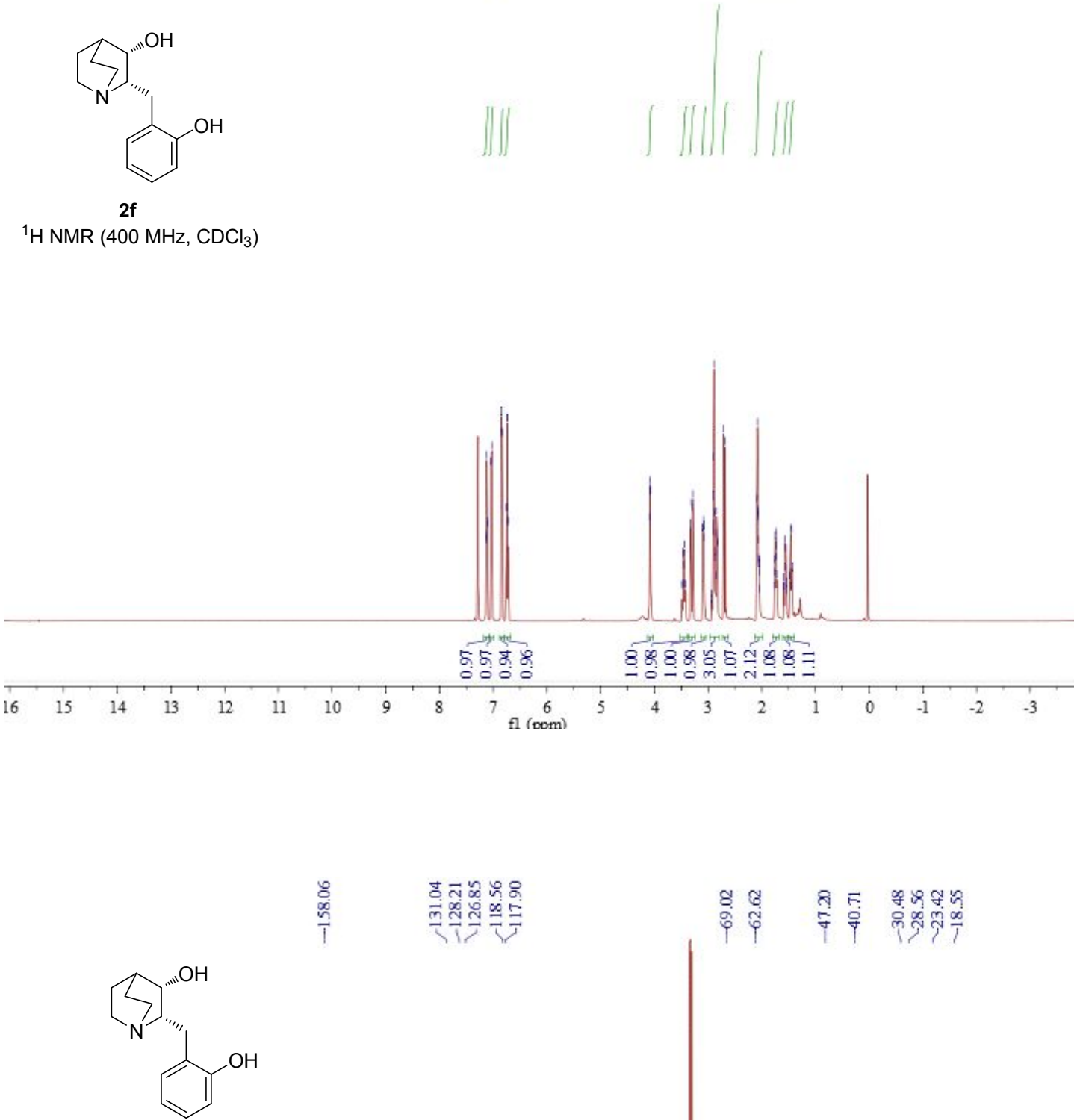

$2 \mathrm{f}$

${ }^{13} \mathrm{C} \mathrm{NMR}\left(151 \mathrm{MHz}, \mathrm{CDCl}_{3}\right)$

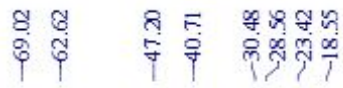

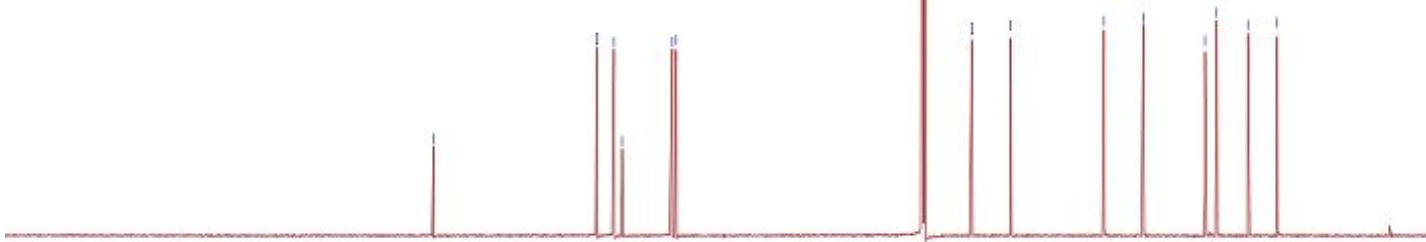

$\begin{array}{lllllllllllllllllllllll}220 & 210 & 200 & 190 & 180 & 170 & 160 & 150 & 140 & 130 & 120 & 110 & 100 & 90 & 80 & 70 & 60 & 50 & 40 & 30 & 20 & 10 & 0\end{array}$ 


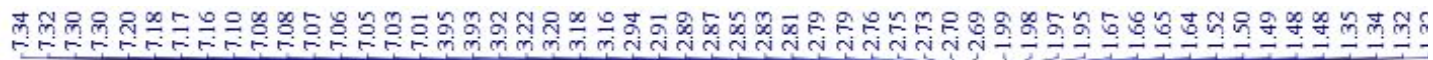
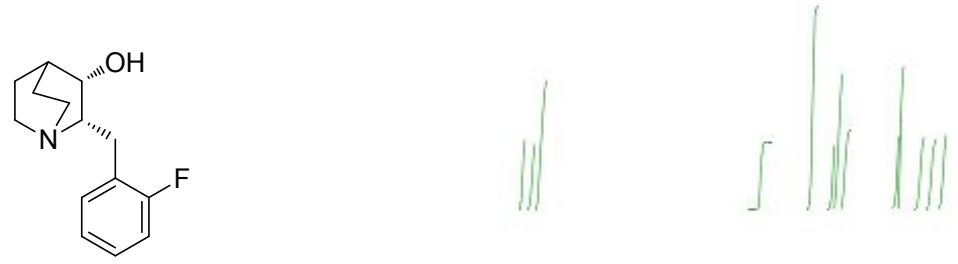

2g

${ }^{1} \mathrm{H} \mathrm{NMR}\left(400 \mathrm{MHz}, \mathrm{CDCl}_{3}\right)$

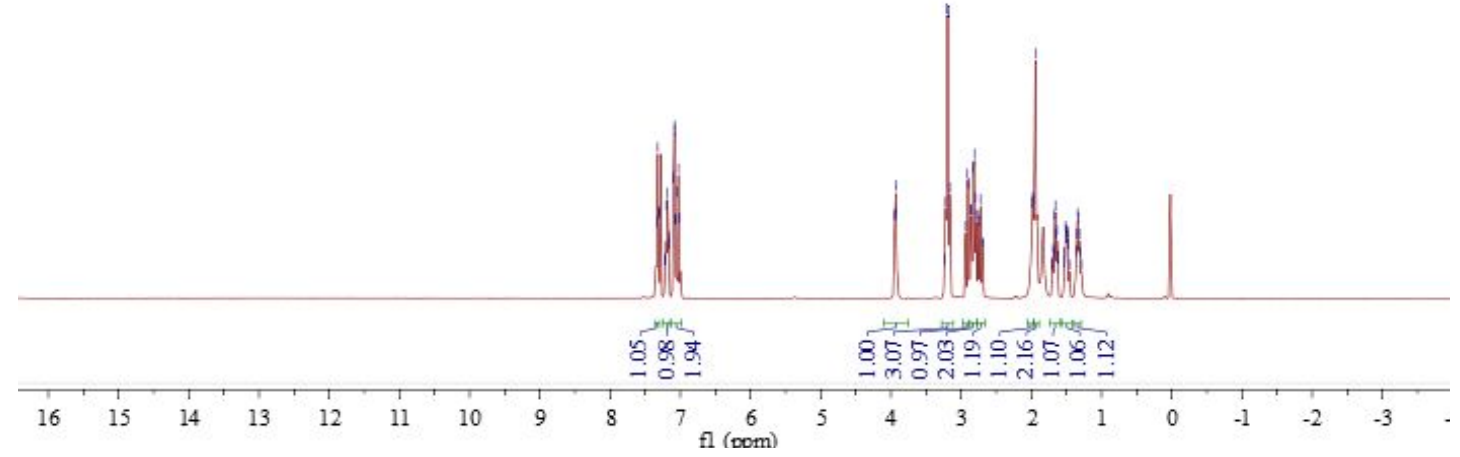

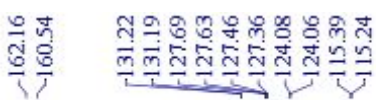

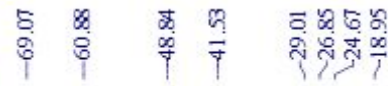

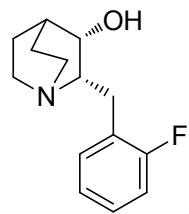

$2 \mathrm{~g}$

${ }^{13} \mathrm{C}$ NMR $\left(151 \mathrm{MHz}, \mathrm{CDCl}_{3}\right)$

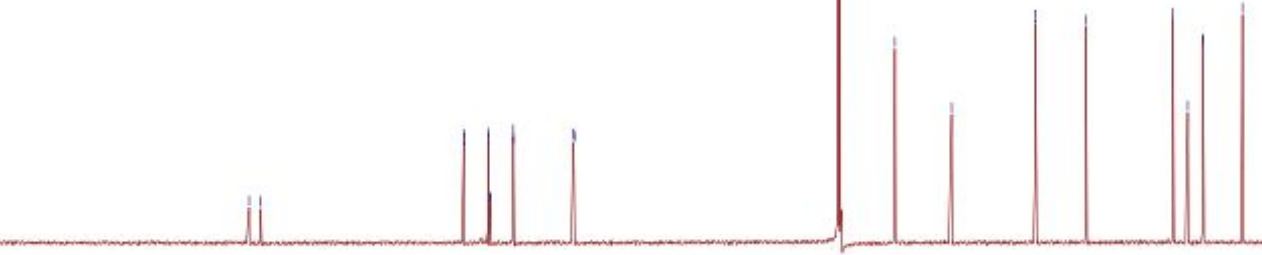

$\begin{array}{lllllllllll}190 & 180 & 170 & 160 & 150 & 140 & 130 & 120 & 110 & 100 & 90 \\ \text { fl (bom) }\end{array}$ 
<smiles>OC1C2CCN(C2)C1Cc1cccc(F)c1</smiles>

2h

${ }^{1} \mathrm{H}$ NMR $\left(400 \mathrm{MHz}, \mathrm{CDCl}_{3}\right)$
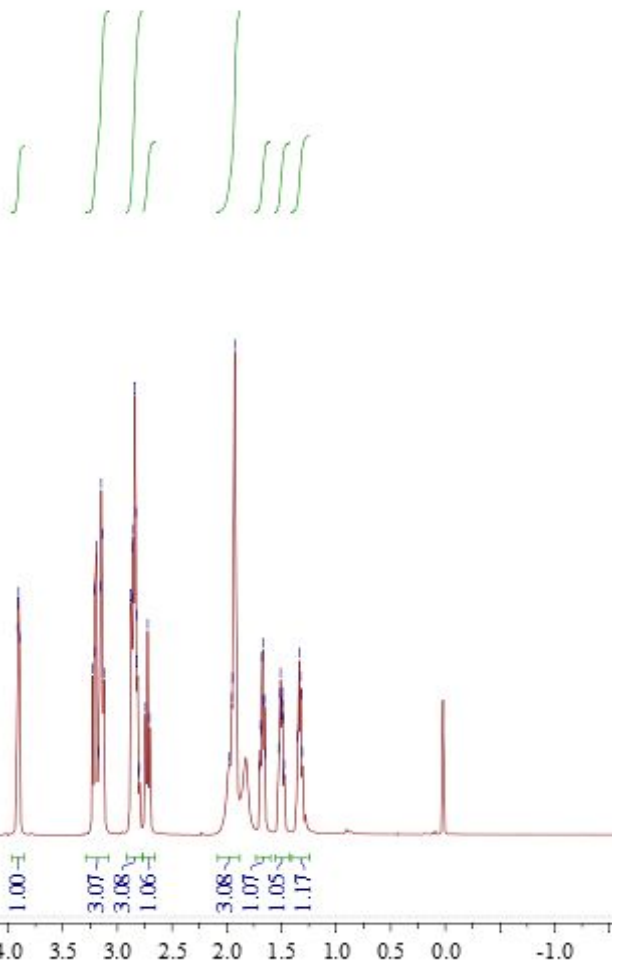

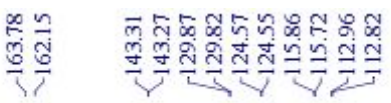

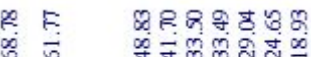

i<smiles>OC1C2CCN1CC2Cc1cccc(F)c1</smiles>

$2 \mathrm{~h}$

${ }^{13} \mathrm{C} \mathrm{NMR}\left(151 \mathrm{MHz}, \mathrm{CDCl}_{3}\right)$

88.8 व

5 fl (pom) 


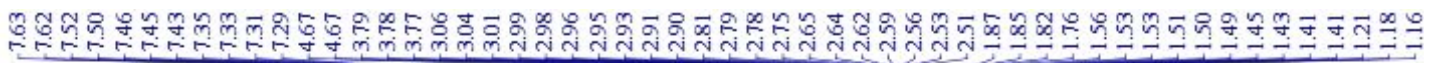
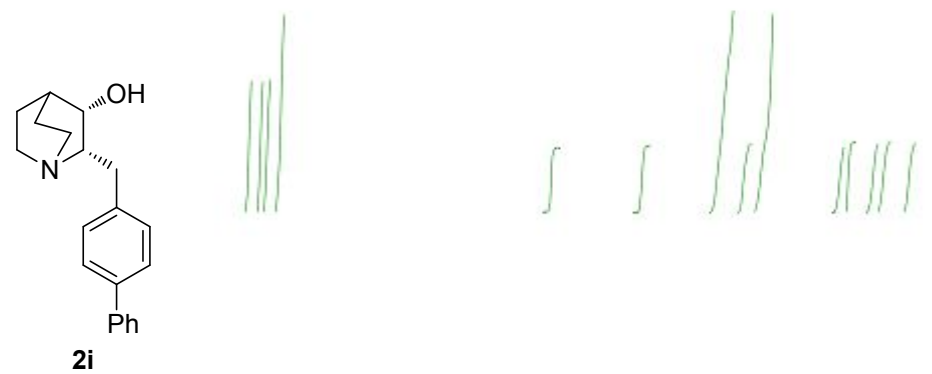

${ }^{1} \mathrm{H}$ NMR $\left(400 \mathrm{MHz}\right.$, DMSO- $\left.d_{6}\right)$
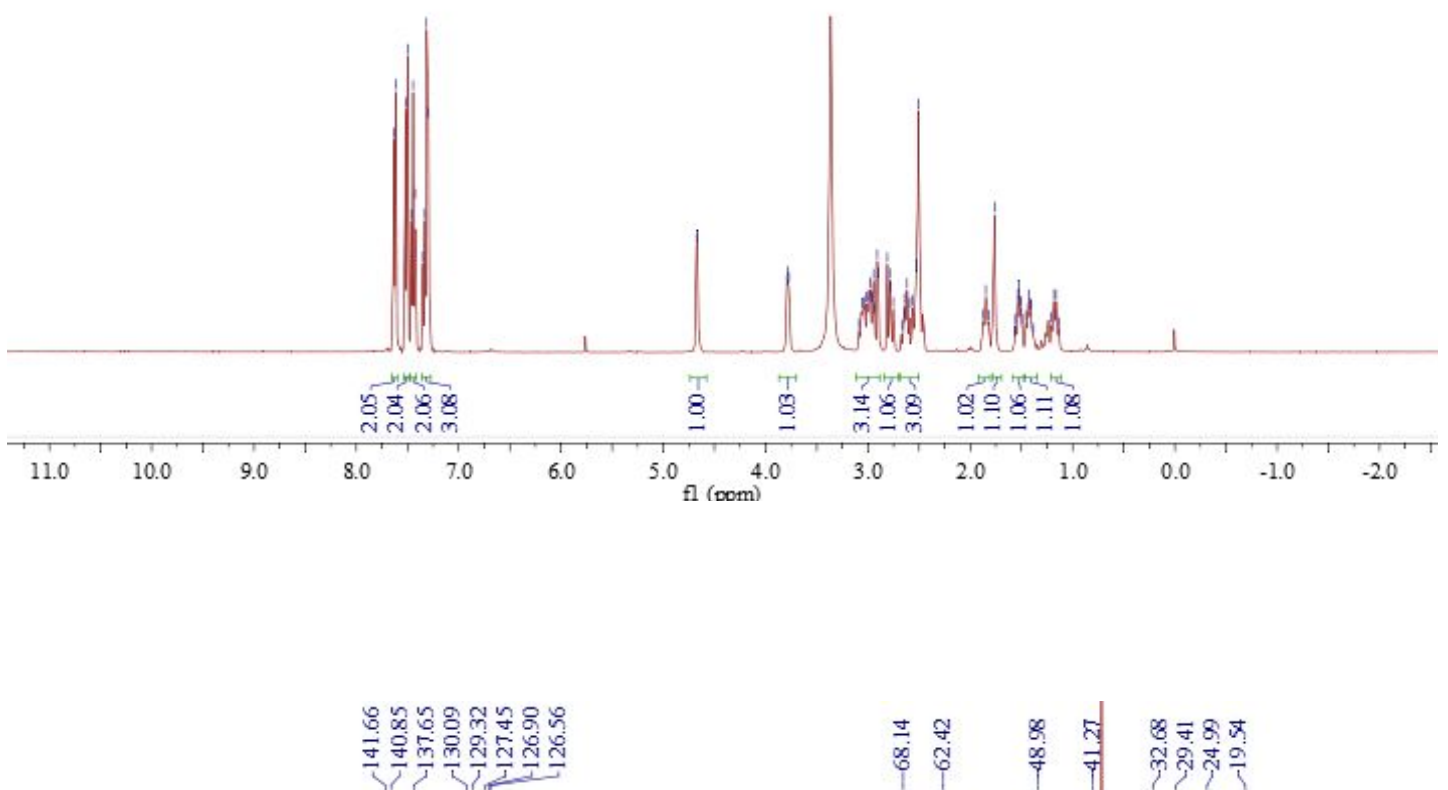

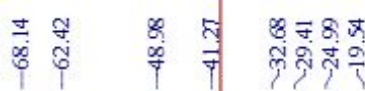

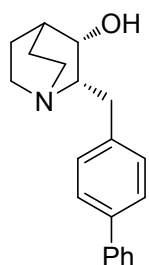

2i

${ }^{13} \mathrm{C}$ NMR $\left(151 \mathrm{MHz}\right.$, DMSO- $\left.d_{6}\right)$

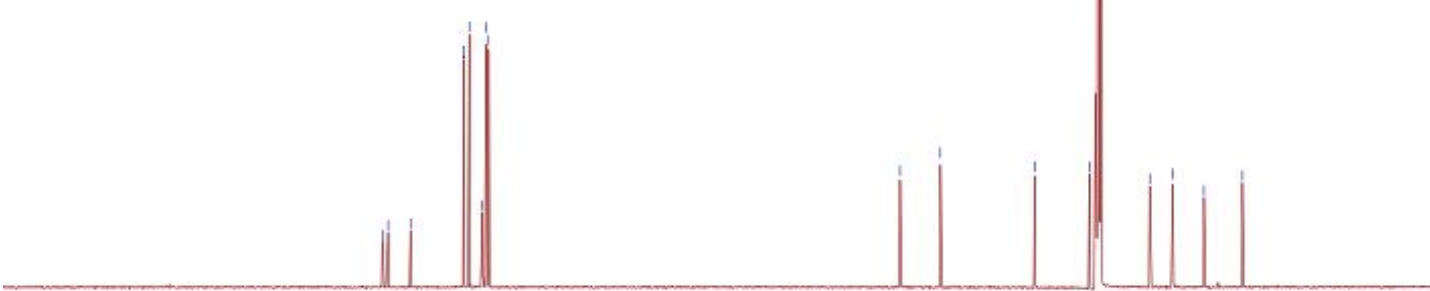

$\begin{array}{llllllllllllllllllll}190 & 180 & 170 & 160 & 150 & 140 & 130 & 120 & 110 & 100 & 90 & 80 & 70 & 60 & 50 & 40 & 30 & 20 & 10 & 0\end{array}$ 


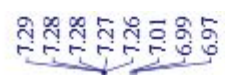

को
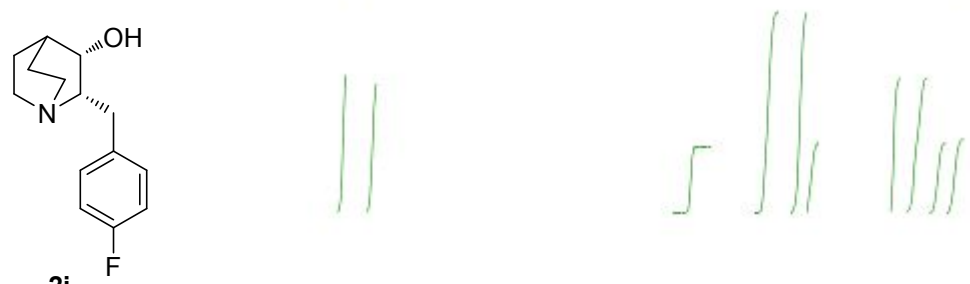

2j

${ }^{1} \mathrm{H}$ NMR $\left(400 \mathrm{MHz}, \mathrm{CDCl}_{3}\right)$

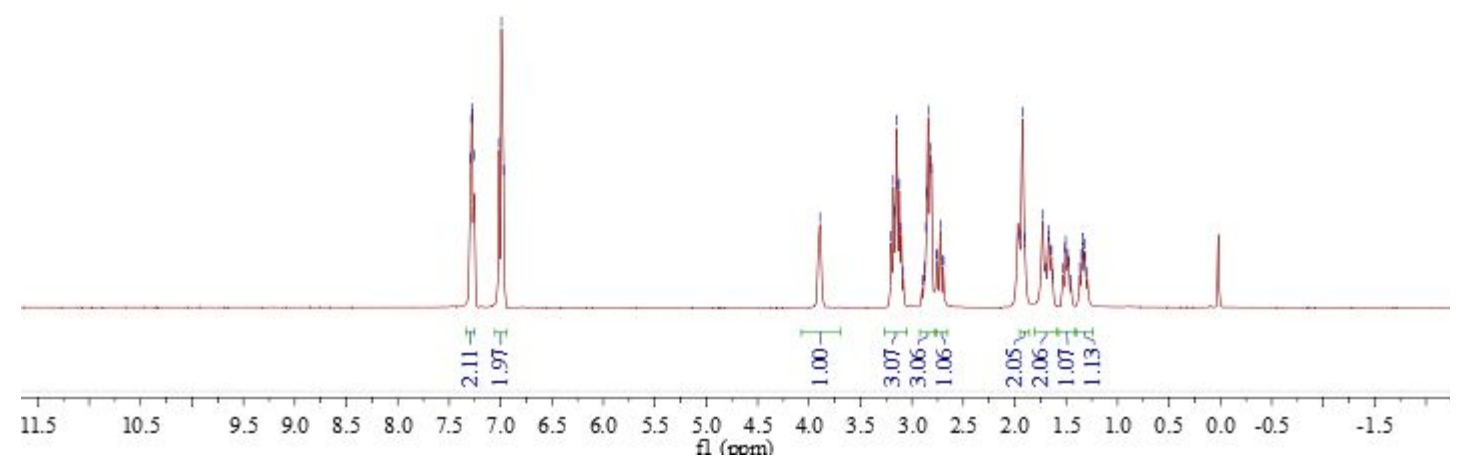

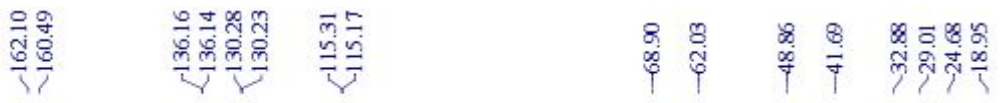

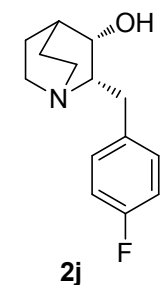

${ }^{13} \mathrm{C}$ NMR (151 MHz, $\mathrm{CDCl}_{3}$ )

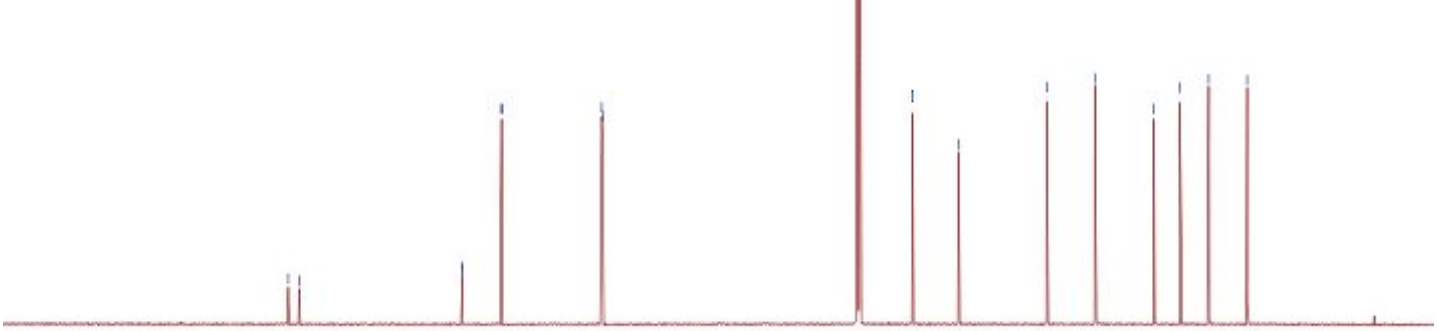

$\begin{array}{lllllllllllllllllllll}200 & 190 & 180 & 170 & 160 & 150 & 140 & 130 & 120 & 110 & 100 & 90 & 80 & 70 & 60 & 50 & 40 & 30 & 20 & 10 & 0\end{array}$ 


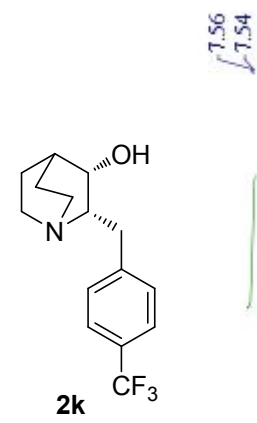

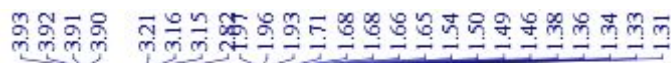

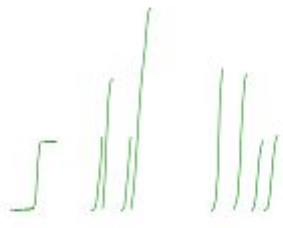

${ }^{1} \mathrm{H}$ NMR $\left(400 \mathrm{MHz}, \mathrm{CDCl}_{3}\right)$
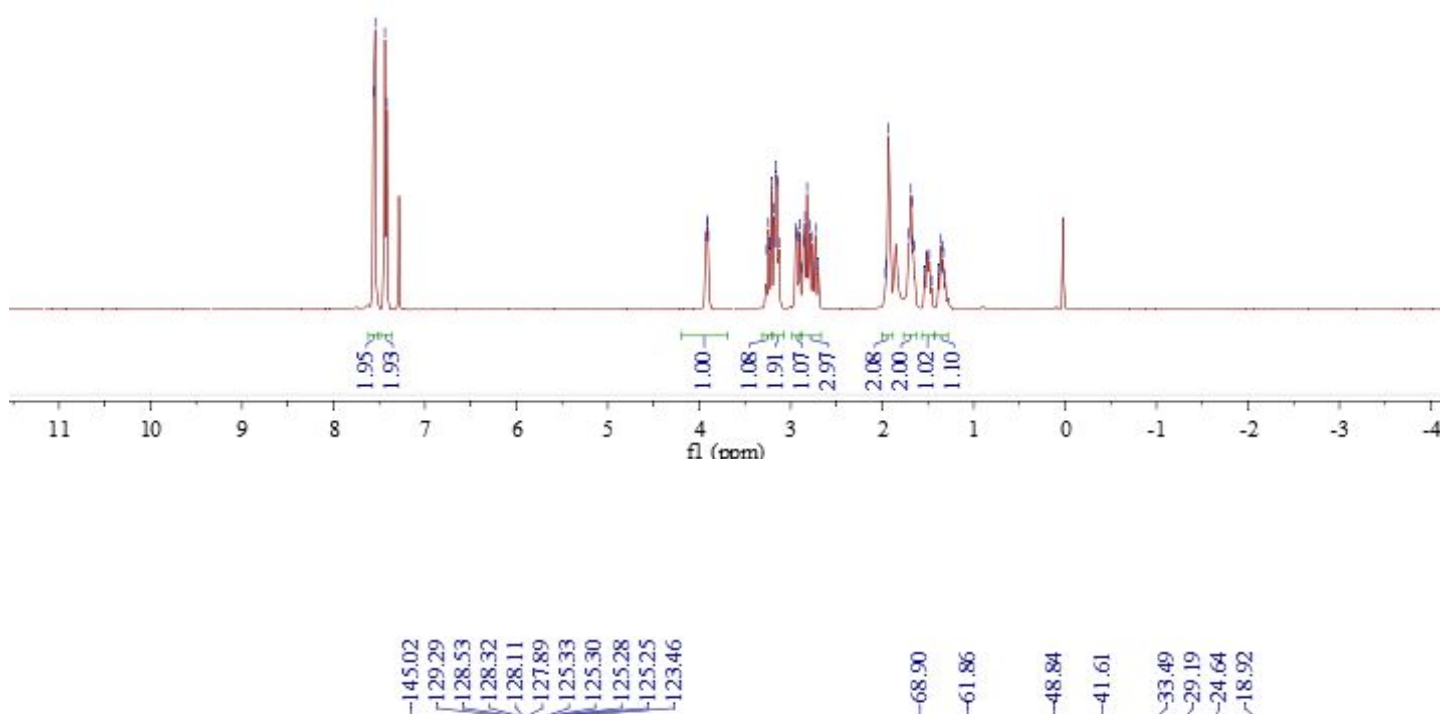

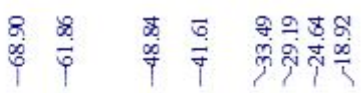

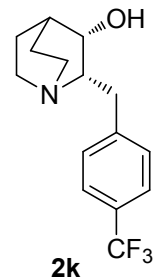

${ }^{13} \mathrm{C}$ NMR $\left(151 \mathrm{MHz}, \mathrm{CDCl}_{3}\right)$

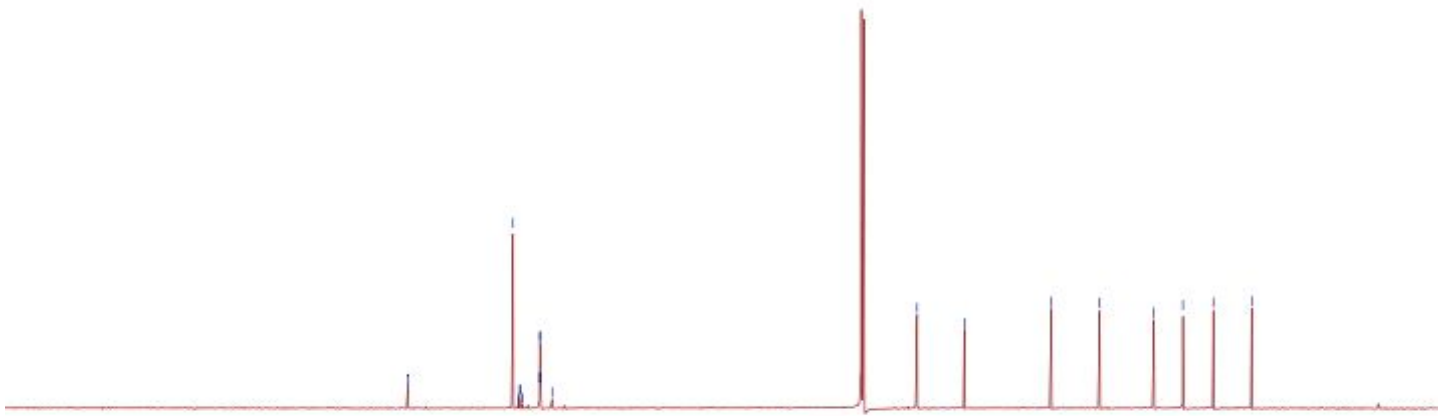

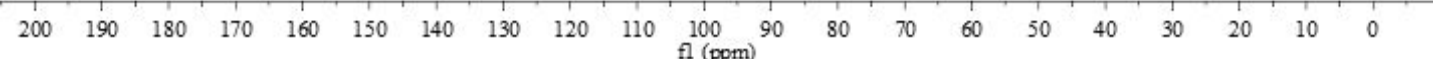




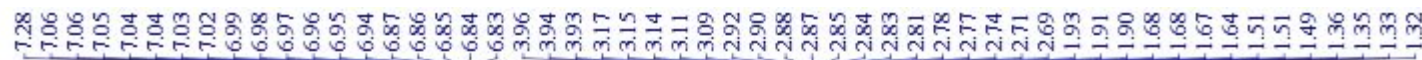
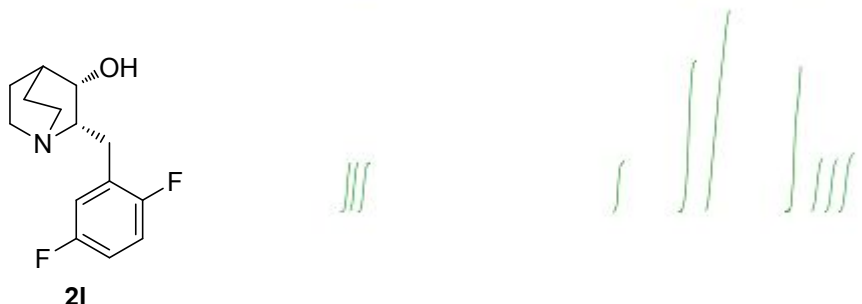

${ }^{1} \mathrm{H}$ NMR $\left(400 \mathrm{MHz}, \mathrm{CDCl}_{3}\right)$
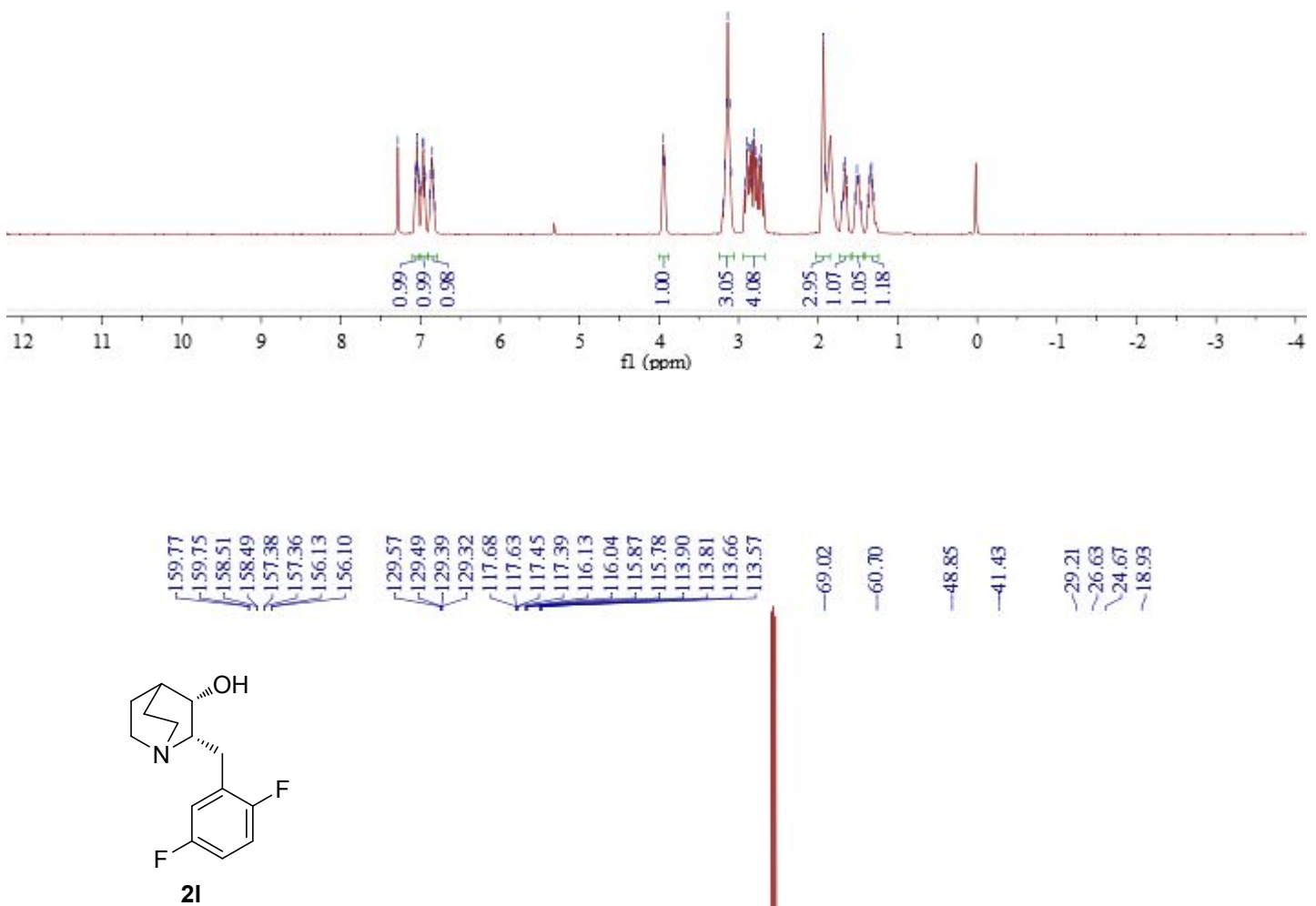

${ }^{13} \mathrm{C}$ NMR $\left(101 \mathrm{MHz}, \mathrm{CDCl}_{3}\right)$

iIII

$\begin{array}{lllllllllll}190 & 180 & 170 & 160 & 150 & 140 & 130 & 120 & 110 & \begin{array}{c}100 \\ \text { fl (ppm) }\end{array}\end{array}$ 


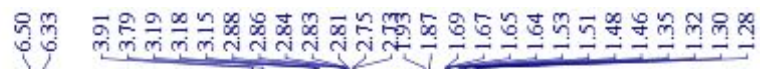

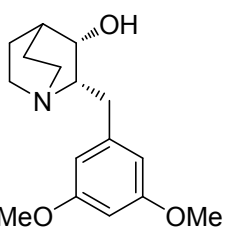

$2 \mathrm{~m}$

${ }^{1} \mathrm{H}$ NMR $\left(400 \mathrm{MHz}, \mathrm{CDCl}_{3}\right)$
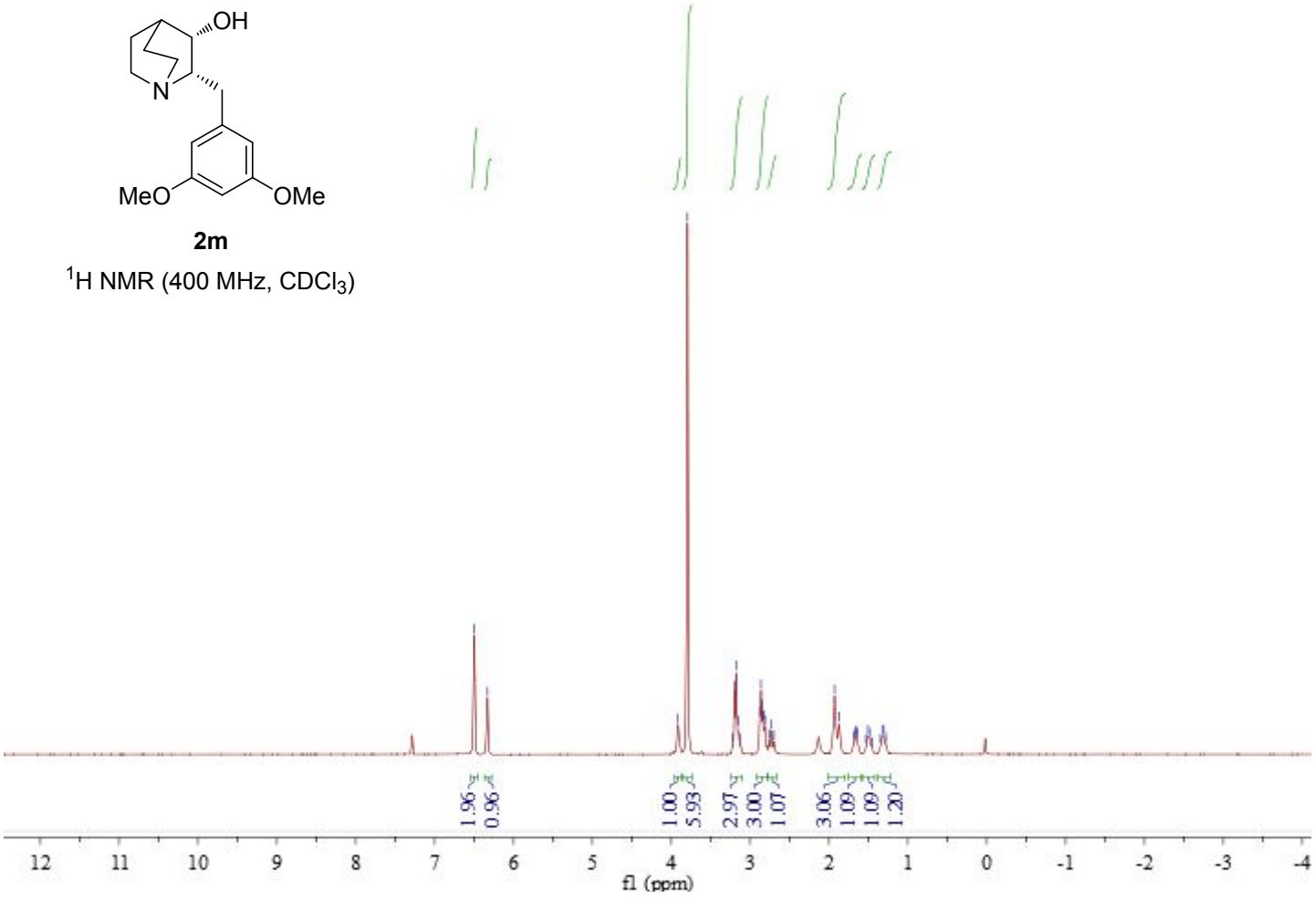

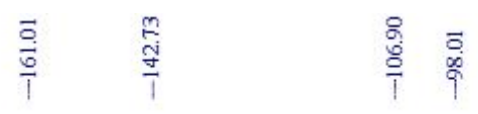

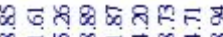

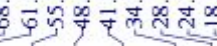

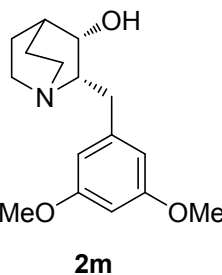

${ }^{13} \mathrm{C}$ NMR $\left(101 \mathrm{MHz}, \mathrm{CDCl}_{3}\right)$

$\begin{array}{llllllllllll}200 & 190 & 180 & 170 & 160 & 150 & 140 & 130 & 120 & 110 & 100 & 90\end{array}$

8070

$60 \quad 50$

$40 \quad 30$

$20 \quad 10$ 


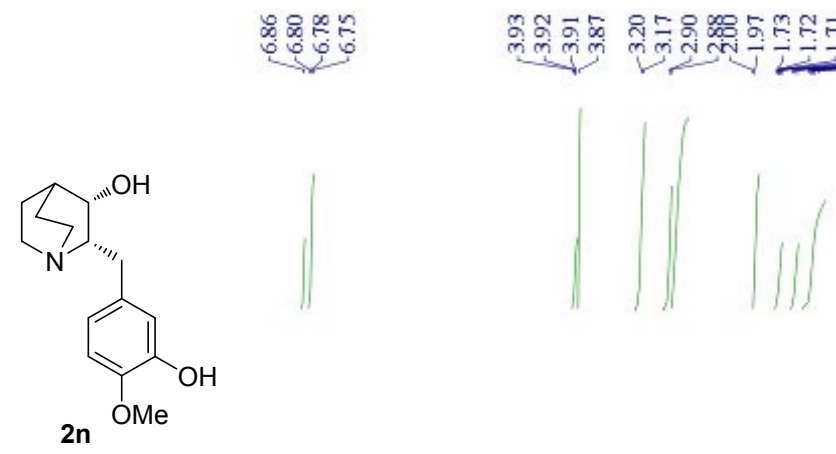

${ }^{1} \mathrm{H}$ NMR $\left(400 \mathrm{MHz}, \mathrm{CDCl}_{3}\right)$

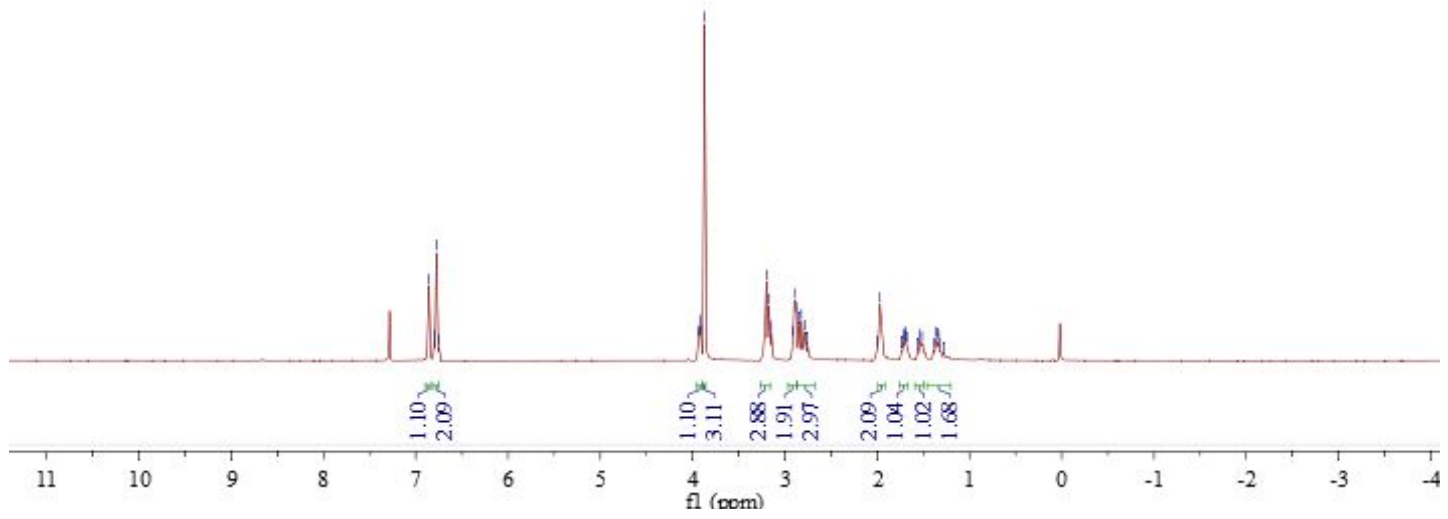

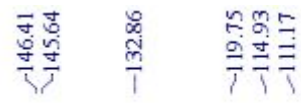

Bร\&R?

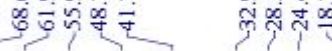

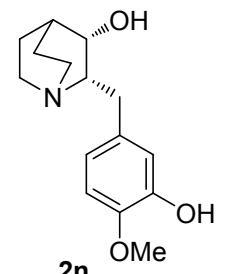

${ }^{13} \mathrm{C}$ NMR $\left(101 \mathrm{MHz}, \mathrm{CDCl}_{3}\right)$
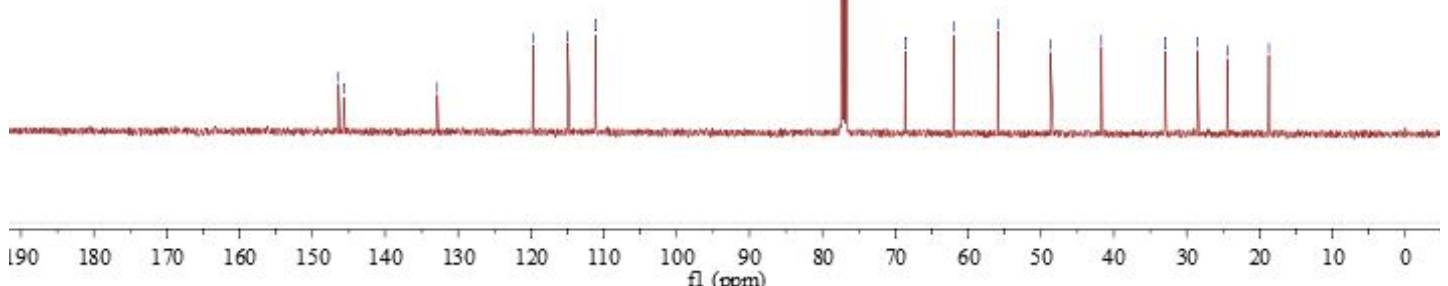

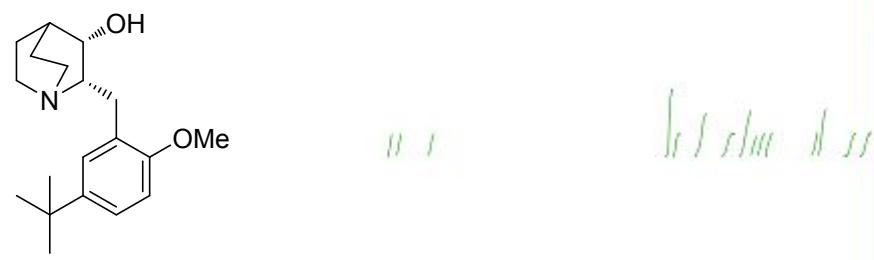

${ }^{1} \mathrm{H}$ NMR $\left(400 \mathrm{MHz}, \mathrm{CDCl}_{3}\right)$
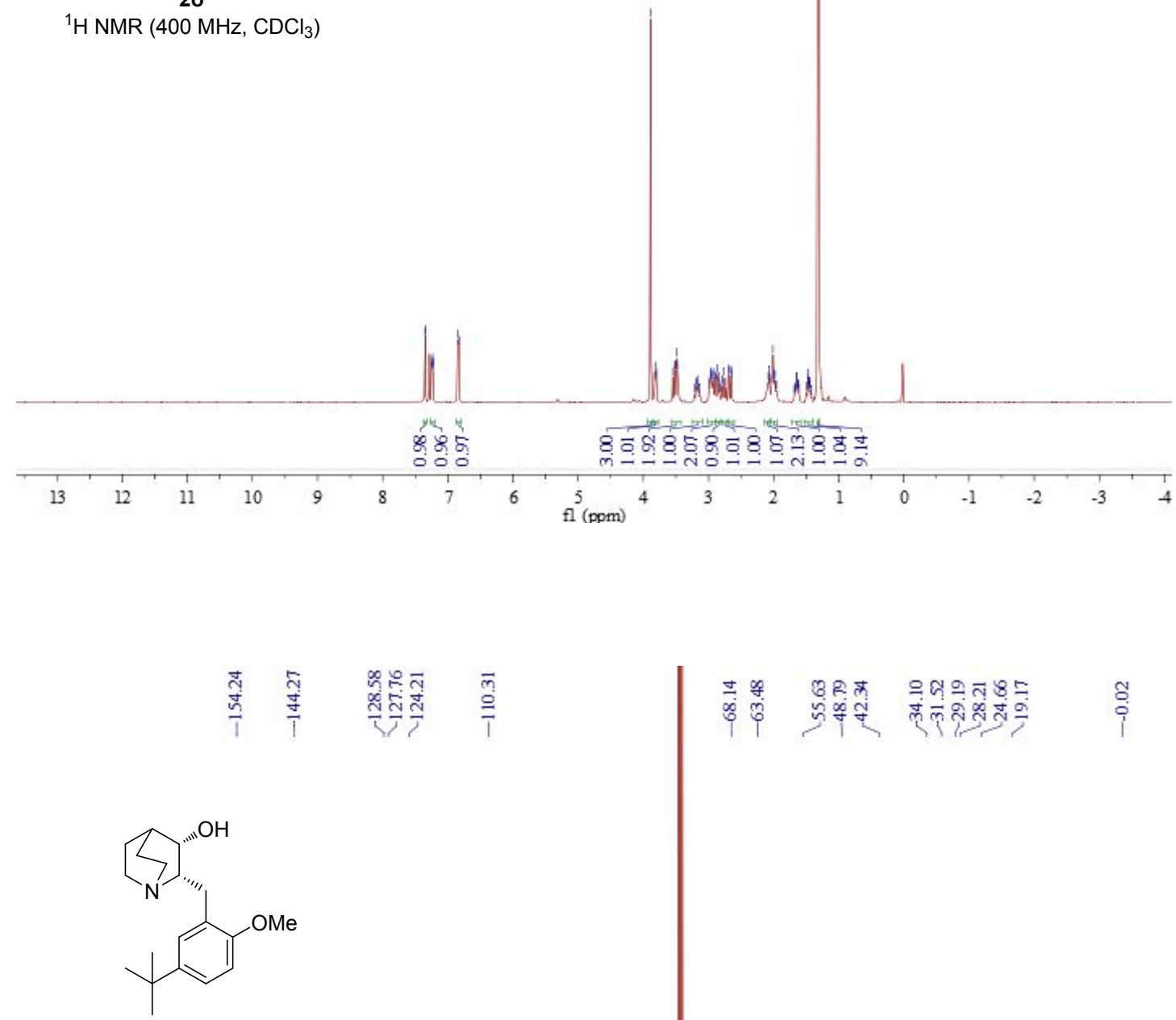

20

${ }^{13} \mathrm{C}$ NMR $\left(101 \mathrm{MHz}, \mathrm{CDCl}_{3}\right)$

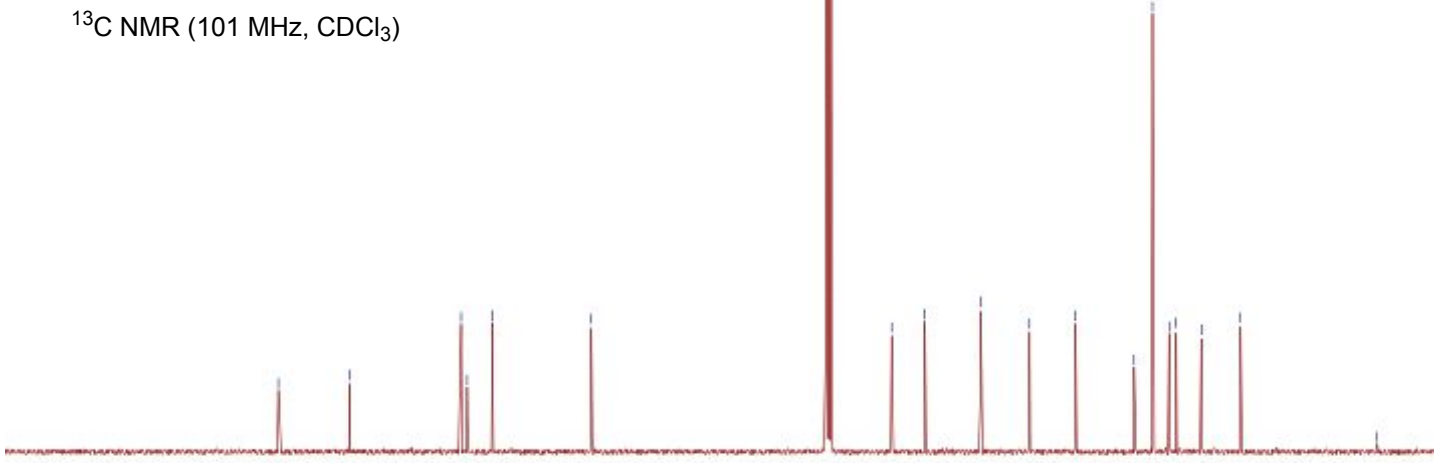

$\begin{array}{llllllllllllllllllllllllllllll}190 & 180 & 170 & 160 & 150 & 140 & 130 & 120 & 110 & 100 & 90 & 80 & 70 & 60 & 50 & 40 & 30 & 20 & 10 & 0\end{array}$ 


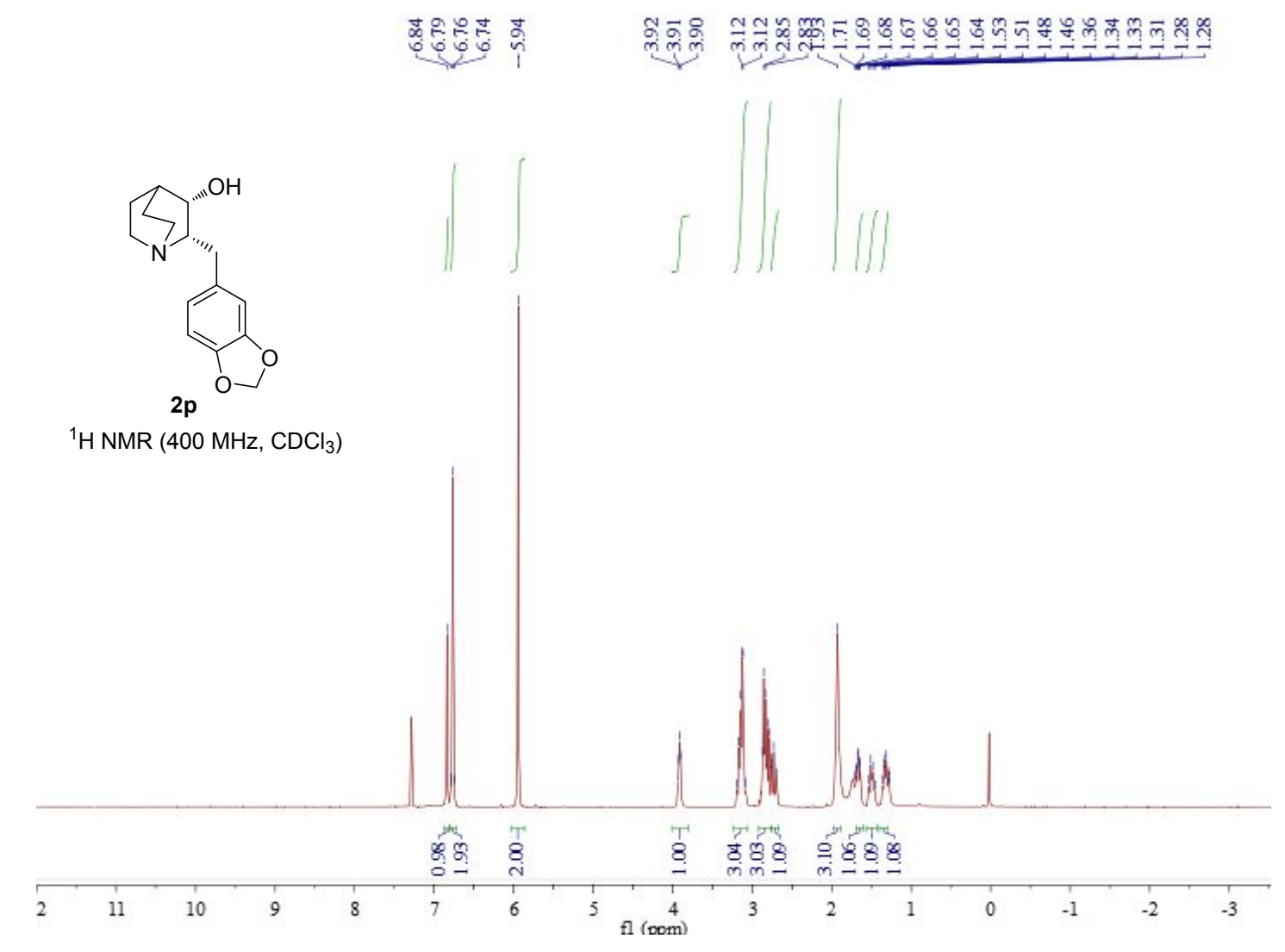

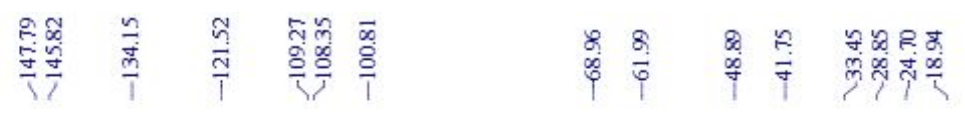

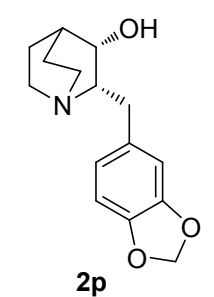

${ }^{13} \mathrm{C} \mathrm{NMR}\left(101 \mathrm{MHz}, \mathrm{CDCl}_{3}\right)$

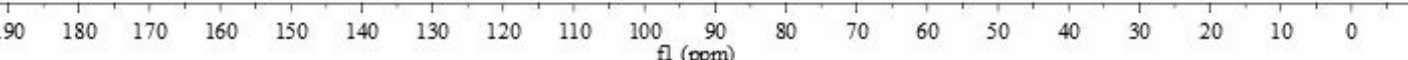




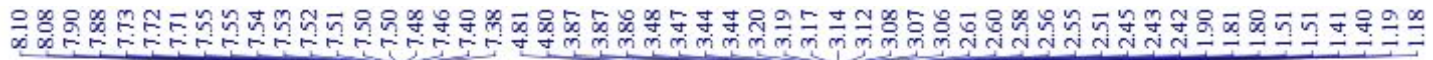
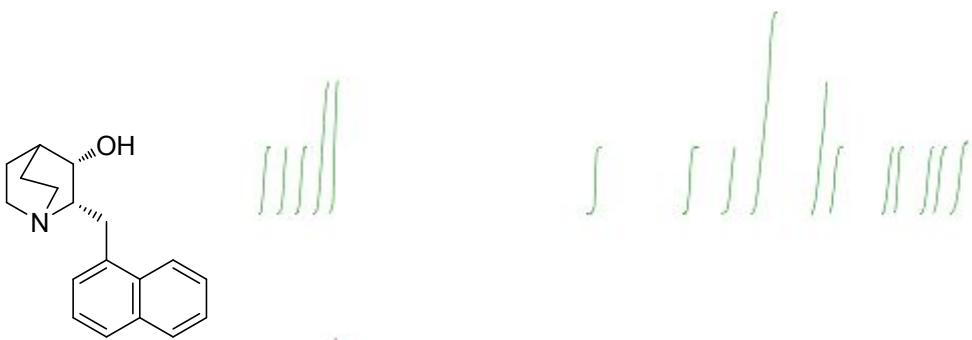

2q

${ }^{1} \mathrm{H}$ NMR $\left(400 \mathrm{MHz}\right.$, DMSO- $\left.d_{6}\right)$
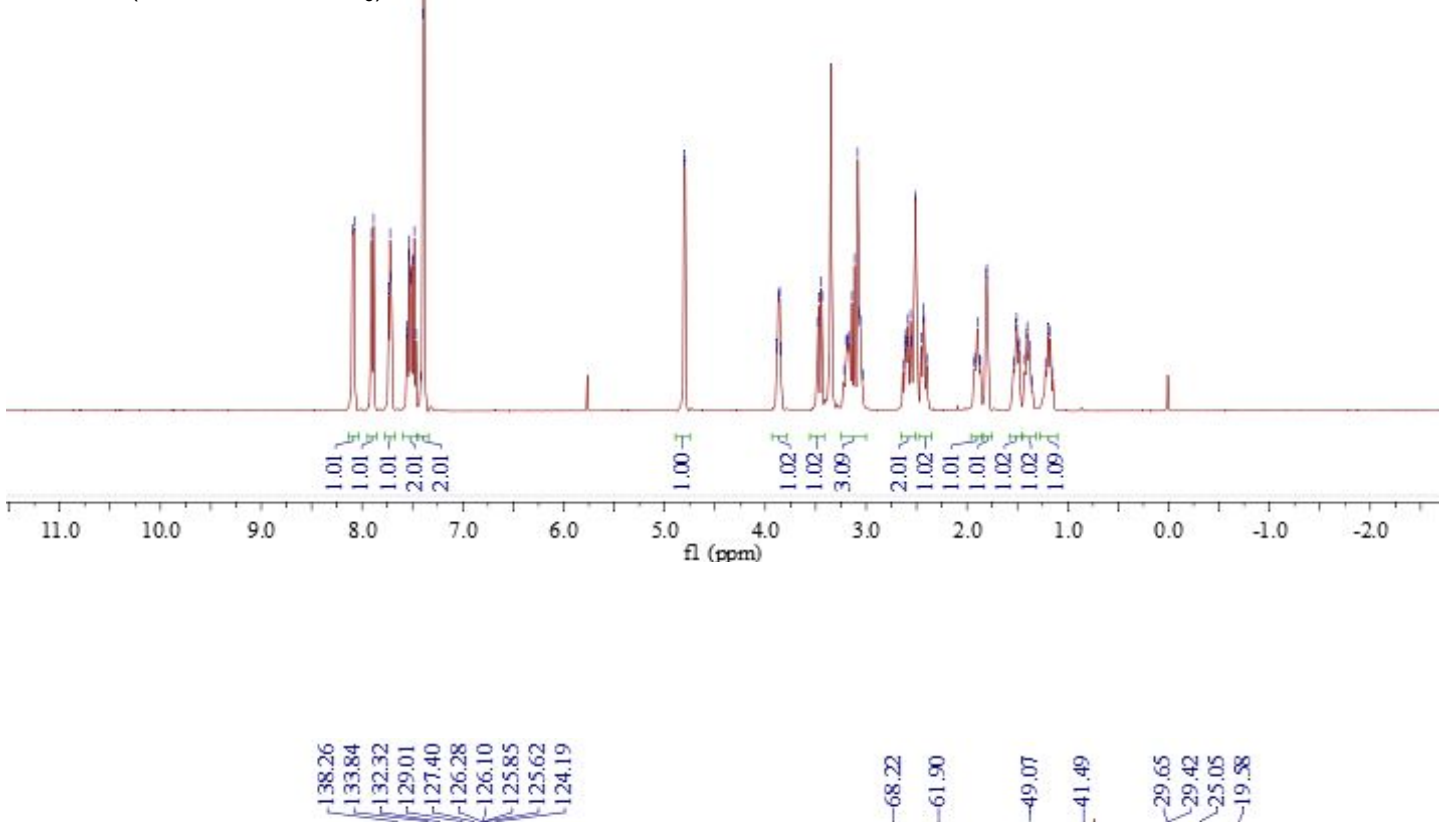

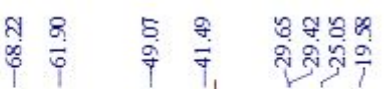

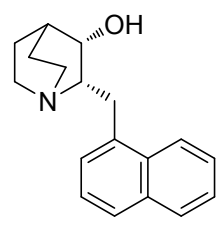

2q

${ }^{13} \mathrm{C}$ NMR $\left(101 \mathrm{MHz}\right.$, DMSO- $\left.d_{6}\right)$

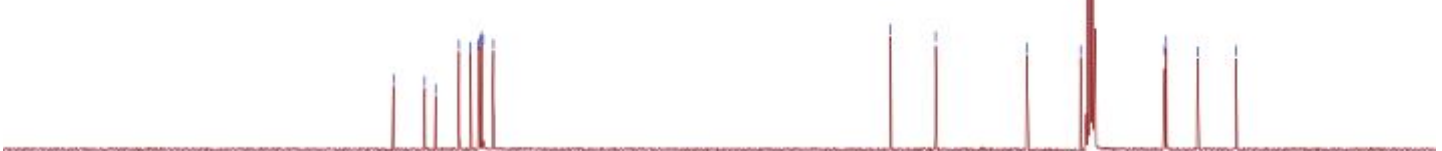

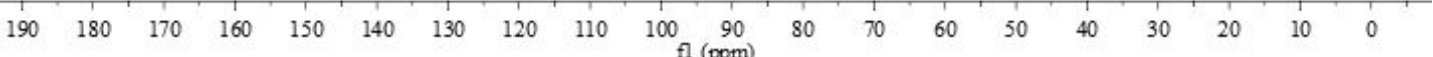



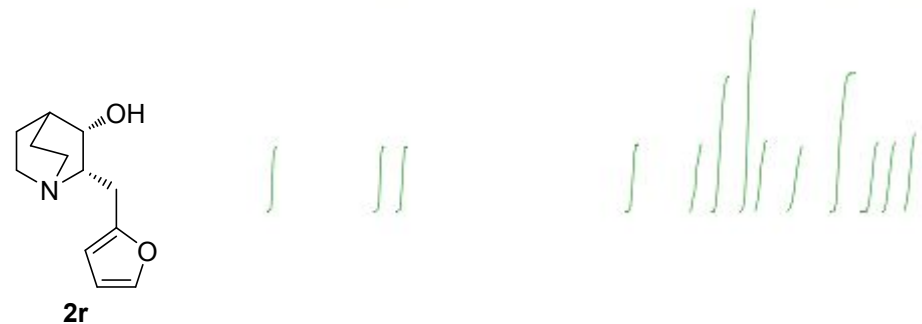

${ }^{1} \mathrm{H}$ NMR $\left(400 \mathrm{MHz}, \mathrm{CDCl}_{3}\right)$
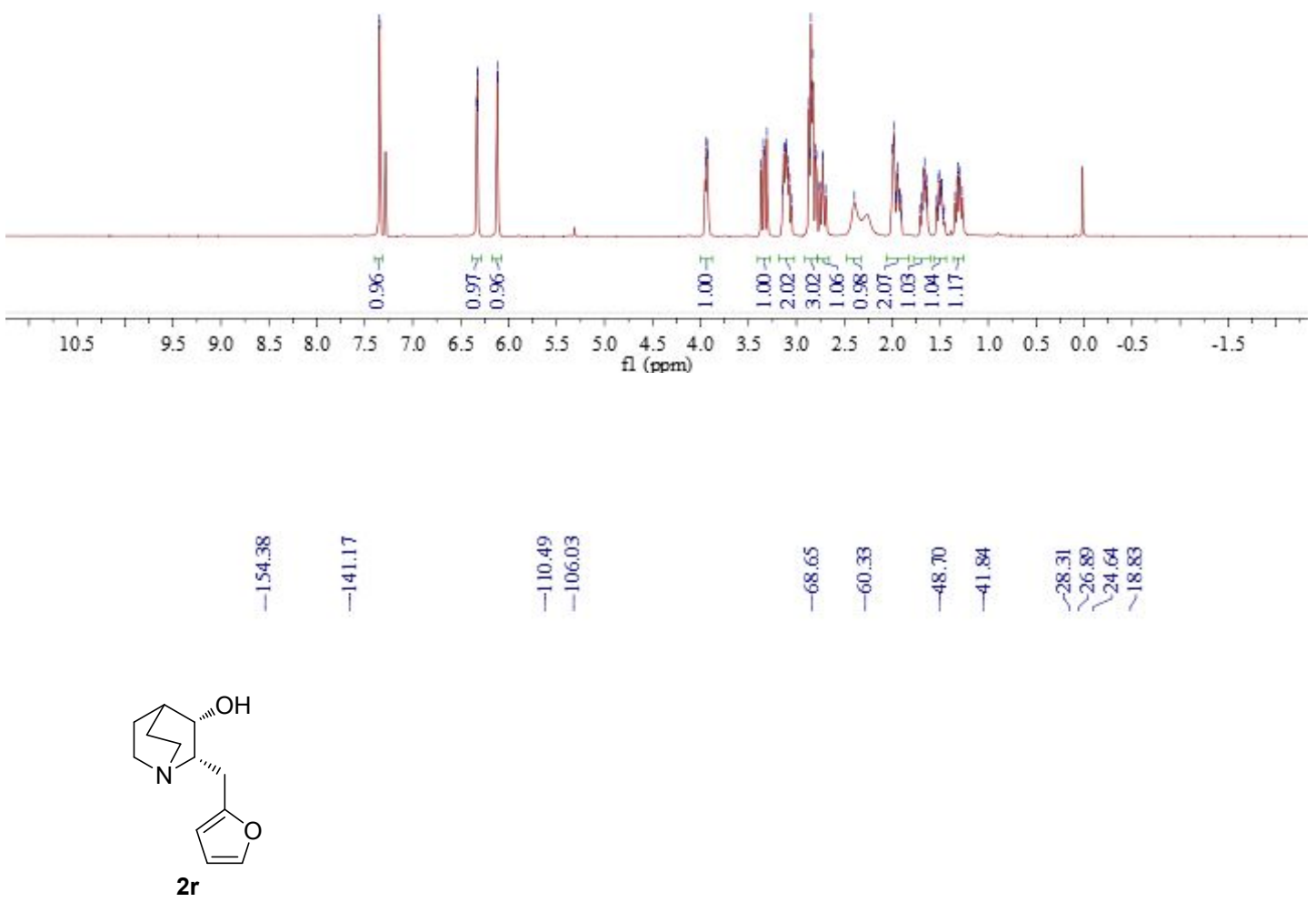

${ }^{13} \mathrm{C} \mathrm{NMR}\left(151 \mathrm{MHz}, \mathrm{CDCl}_{3}\right)$ 

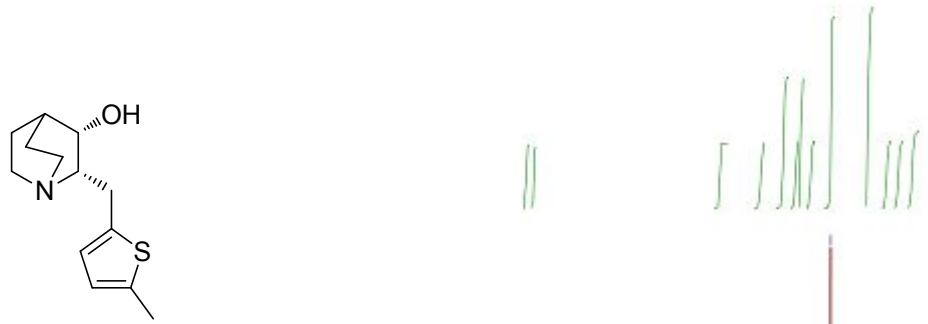

2s

${ }^{1} \mathrm{H}$ NMR $\left(400 \mathrm{MHz}, \mathrm{CDCl}_{3}\right)$

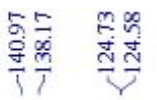

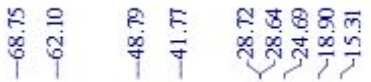

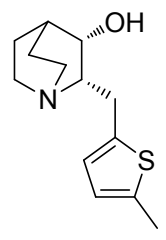

2s

${ }^{13} \mathrm{C}$ NMR $\left(151 \mathrm{MHz}, \mathrm{CDCl}_{3}\right)$

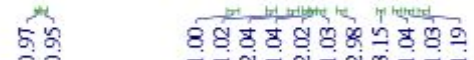
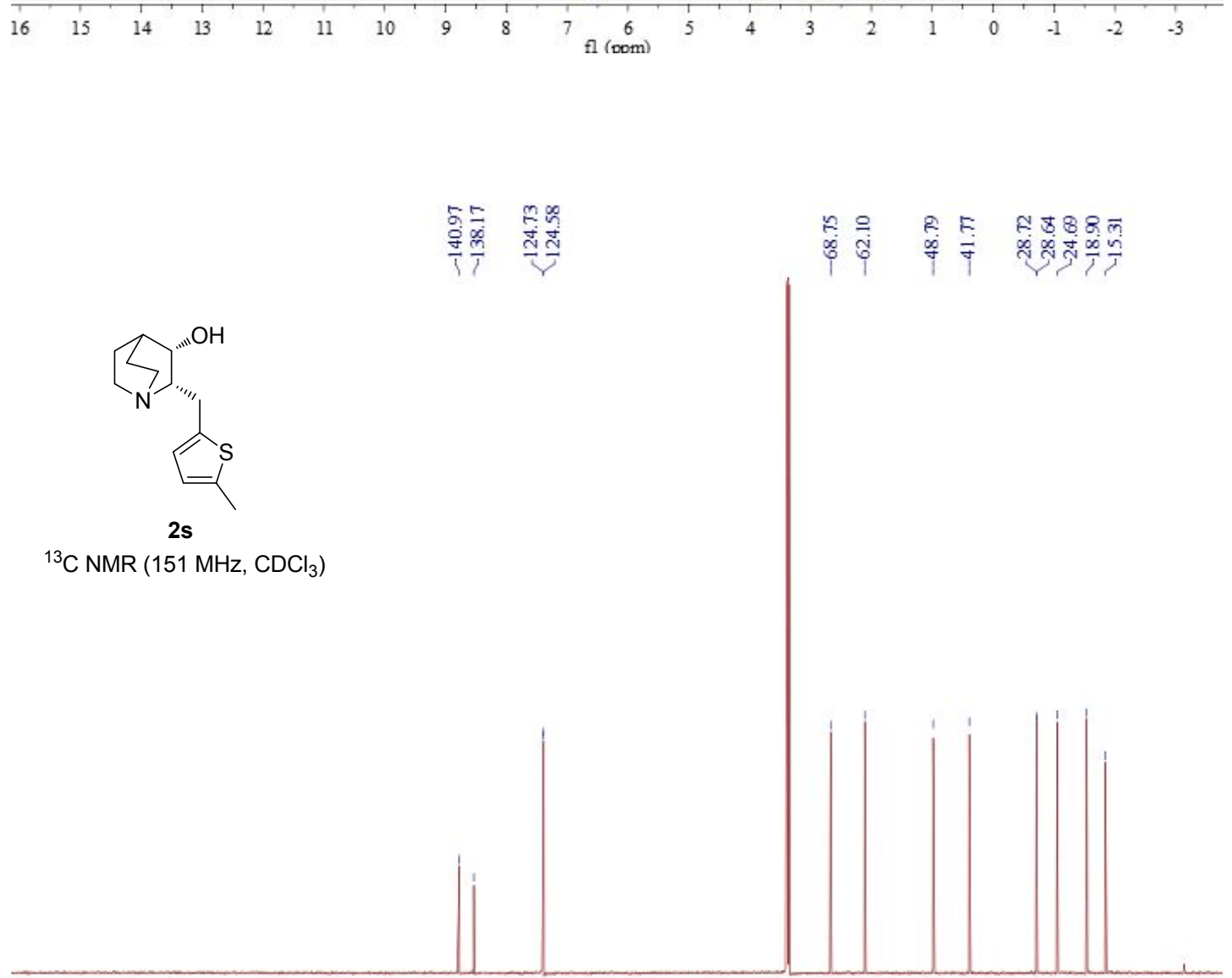

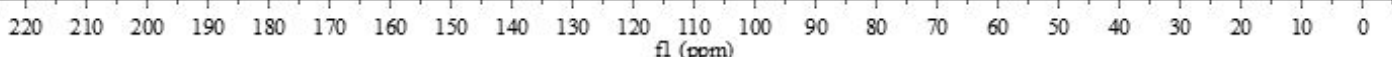




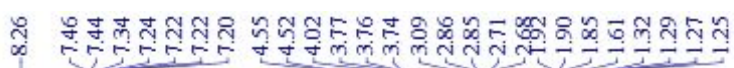
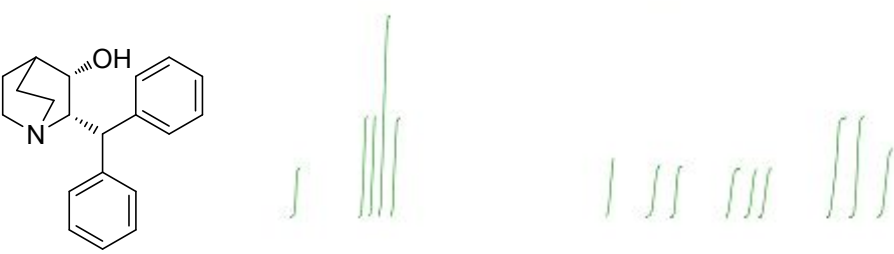

$2 t$

${ }^{1} \mathrm{H}$ NMR $\left(400 \mathrm{MHz}\right.$, DMSO- $\left.d_{6}\right)$
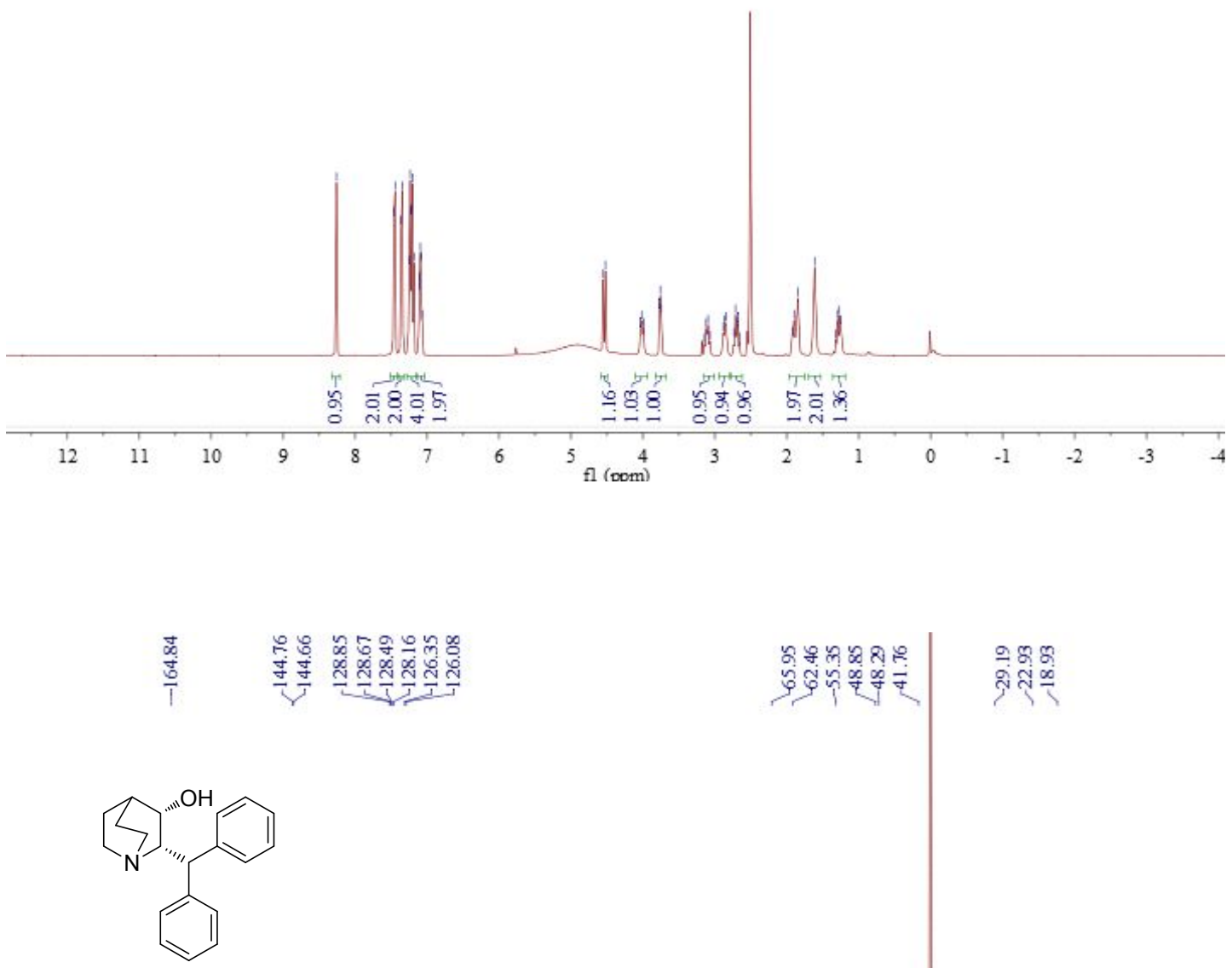

2t

${ }^{13} \mathrm{C}$ NMR $\left(151 \mathrm{MHz}\right.$, DMSO- $\left.d_{6}\right)$

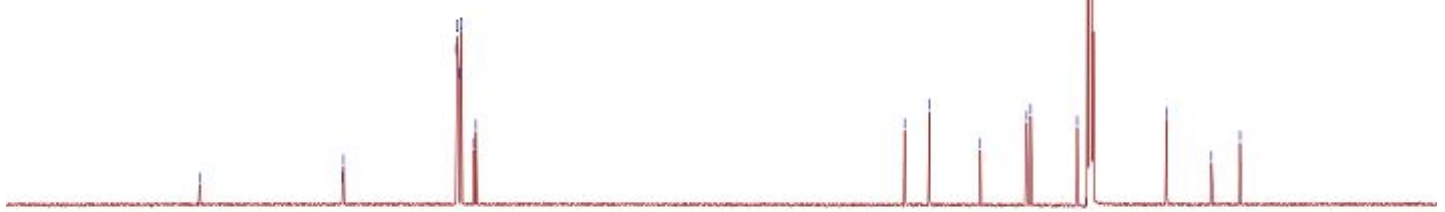

190

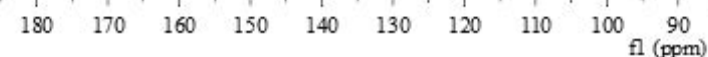

$\begin{array}{lllllllll}1 & 1 & 1 & 1 & 1 & 1 & 1 & 1 \\ 80 & 70 & 60 & 50 & 40 & 30 & 20 & 10 & 0\end{array}$




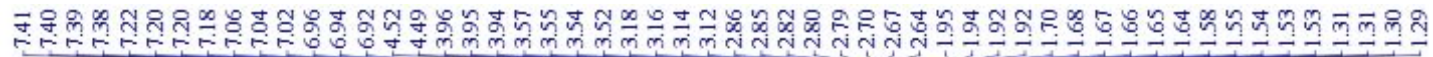
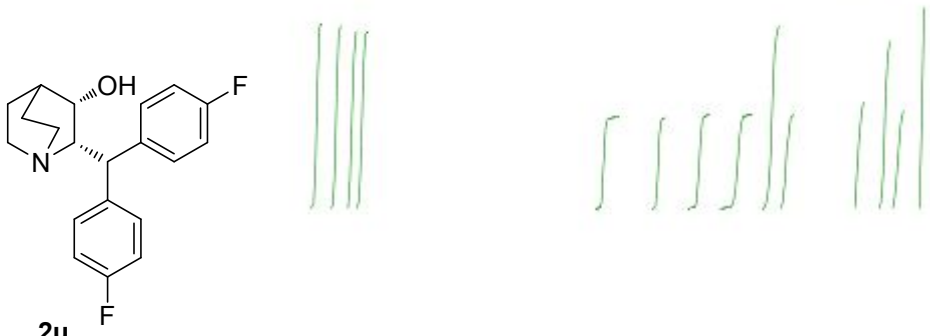

${ }^{1} \mathrm{H} \mathrm{NMR}\left(400 \mathrm{MHz}, \mathrm{CDCl}_{3}\right)$
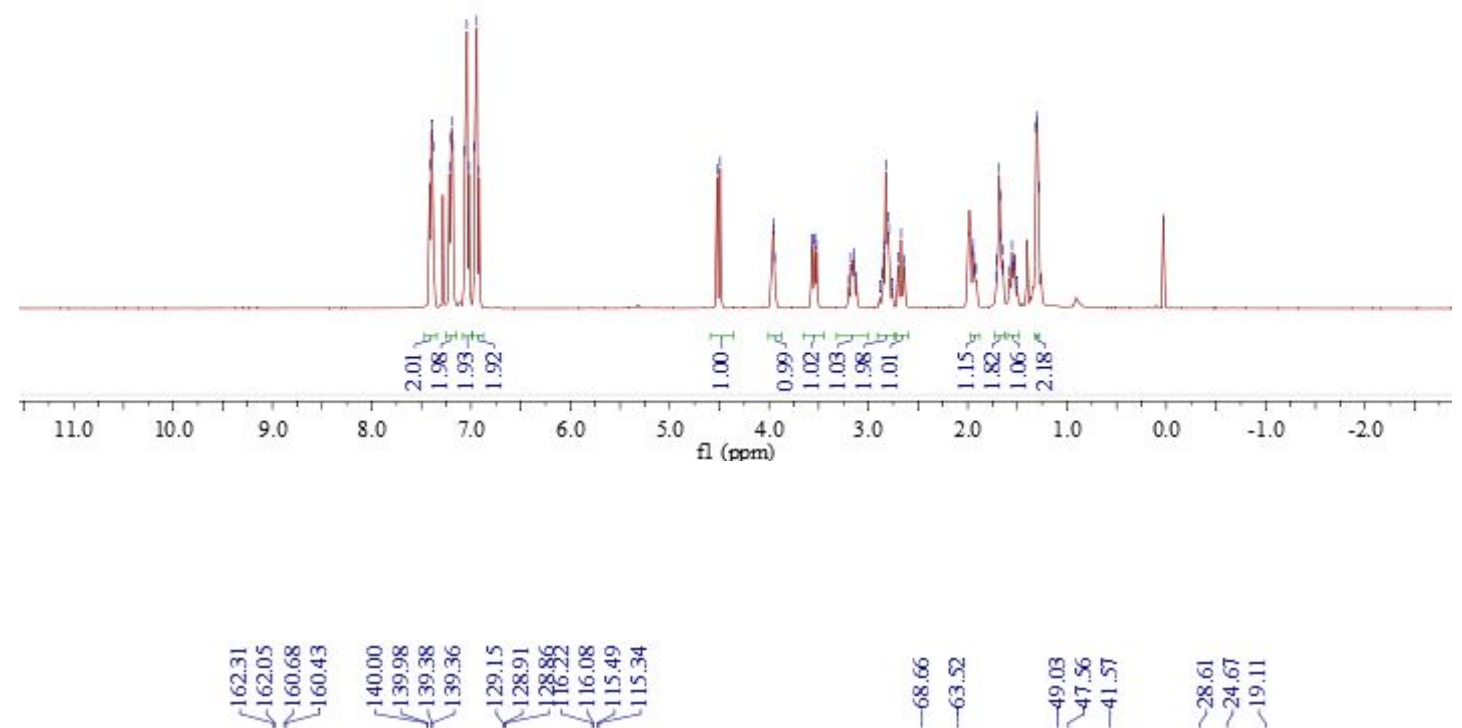

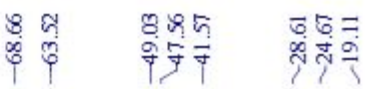

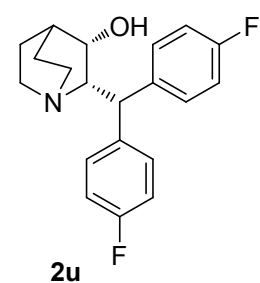

$\left.{ }^{13} \mathrm{C} \mathrm{NMR} \mathrm{(151} \mathrm{MHz,} \mathrm{CDCl}_{3}\right)$

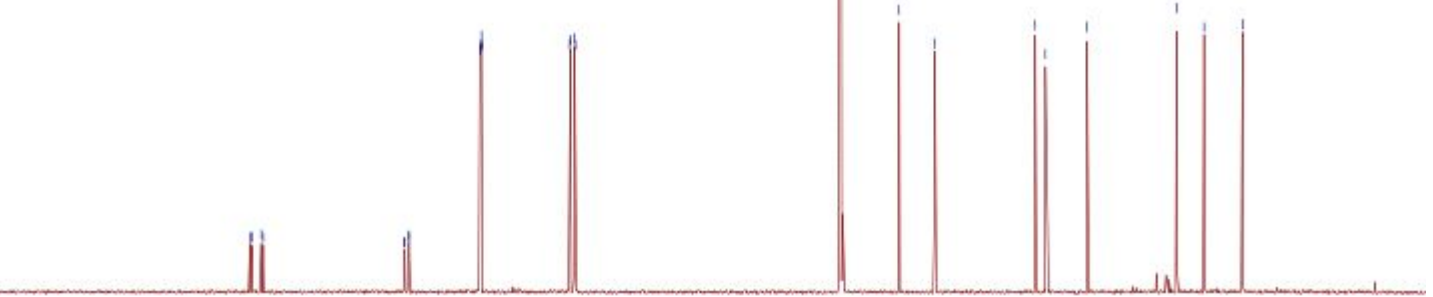

$\begin{array}{lllllllllll}190 & 180 & 170 & 160 & 150 & 140 & 130 & 120 & 110 & 100 & 90 \\ \mathrm{fl}(\mathrm{Dom})\end{array}$ 


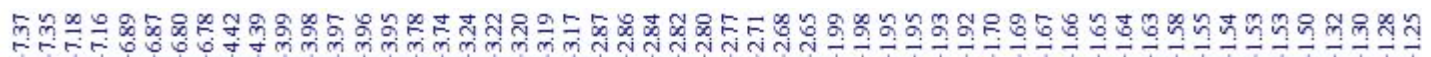<smiles>COc1ccc(C(c2ccc(OC)cc2)[C@@H]2C[C@H]3CCN2C3)cc1</smiles>

2v

${ }^{1} \mathrm{H}$ NMR $\left(400 \mathrm{MHz}, \mathrm{CDCl}_{3}\right)$
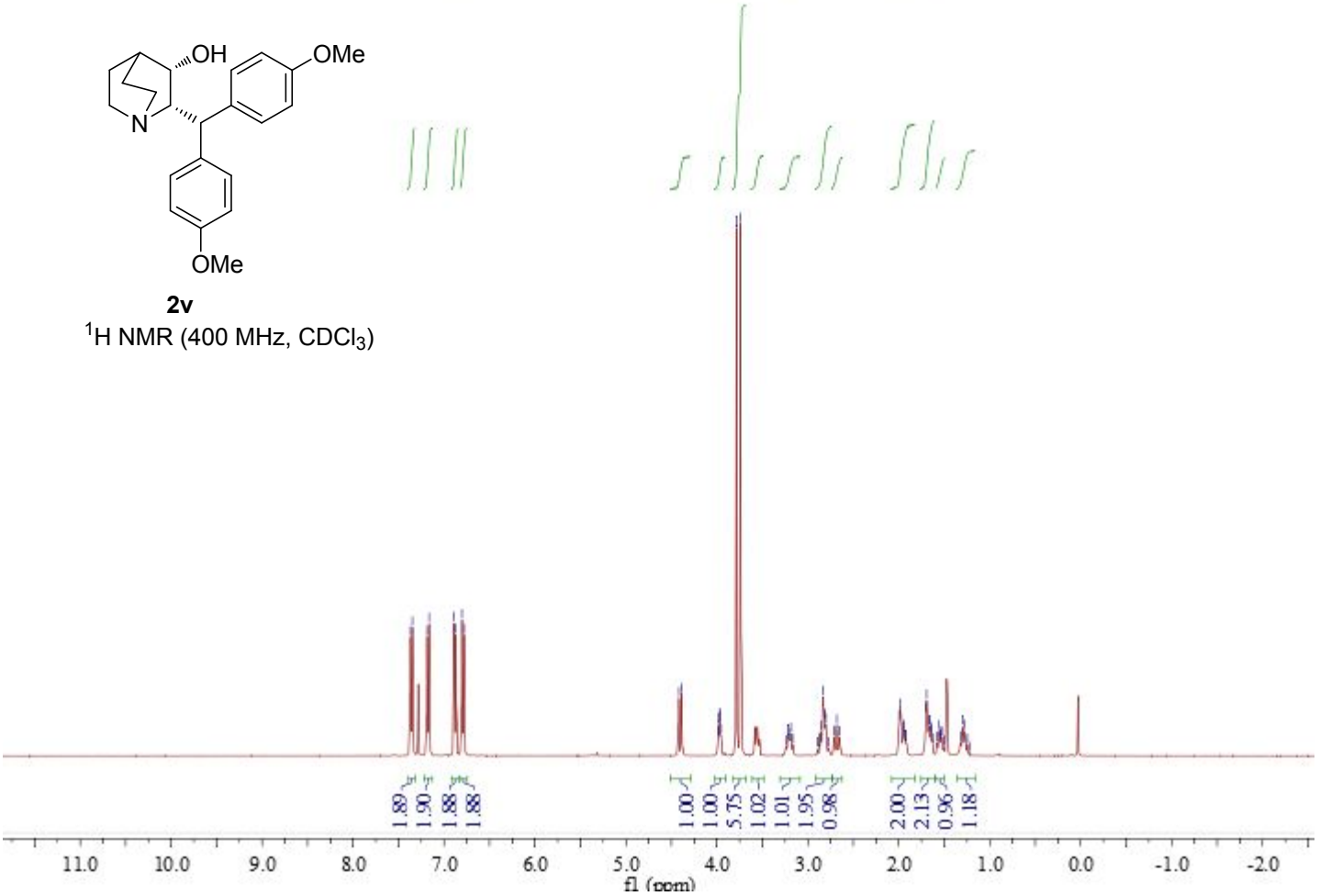

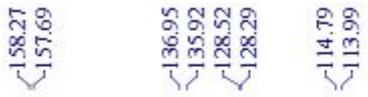

8. 8 8ुष

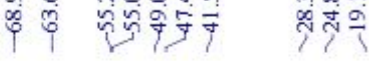

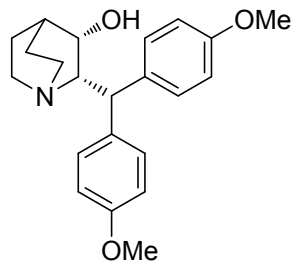

2v

${ }^{13} \mathrm{C}$ NMR $\left(151 \mathrm{MHz}, \mathrm{CDCl}_{3}\right)$

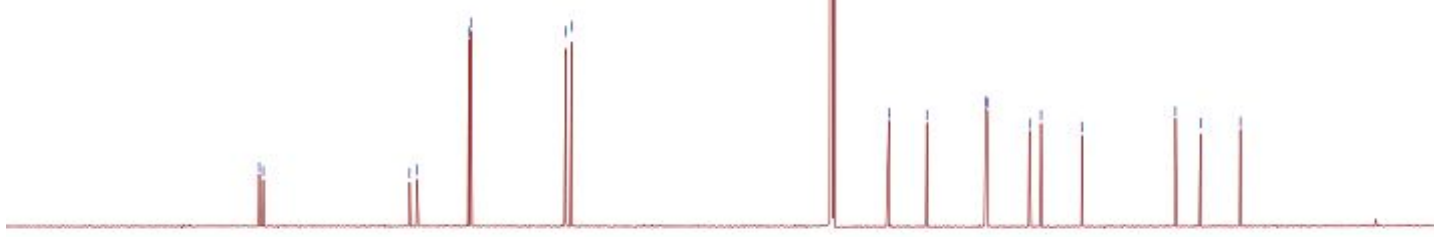

$\begin{array}{lllllllllll}190 & 180 & 170 & 160 & 150 & 140 & 130 & 120 & 110 & 100 & 90\end{array}$

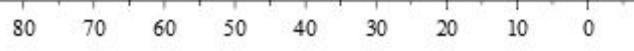



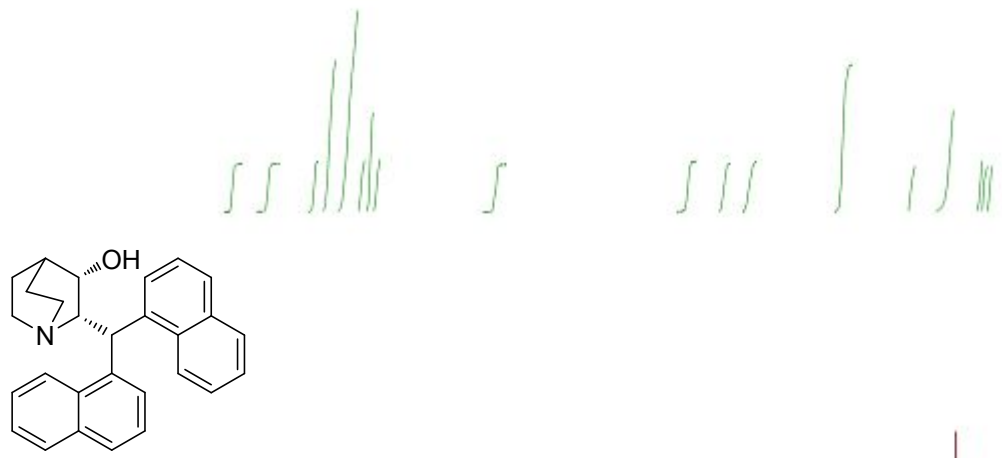

2w

${ }^{1} \mathrm{H}$ NMR $\left(400 \mathrm{MHz}, \mathrm{CDCl}_{3}\right)$

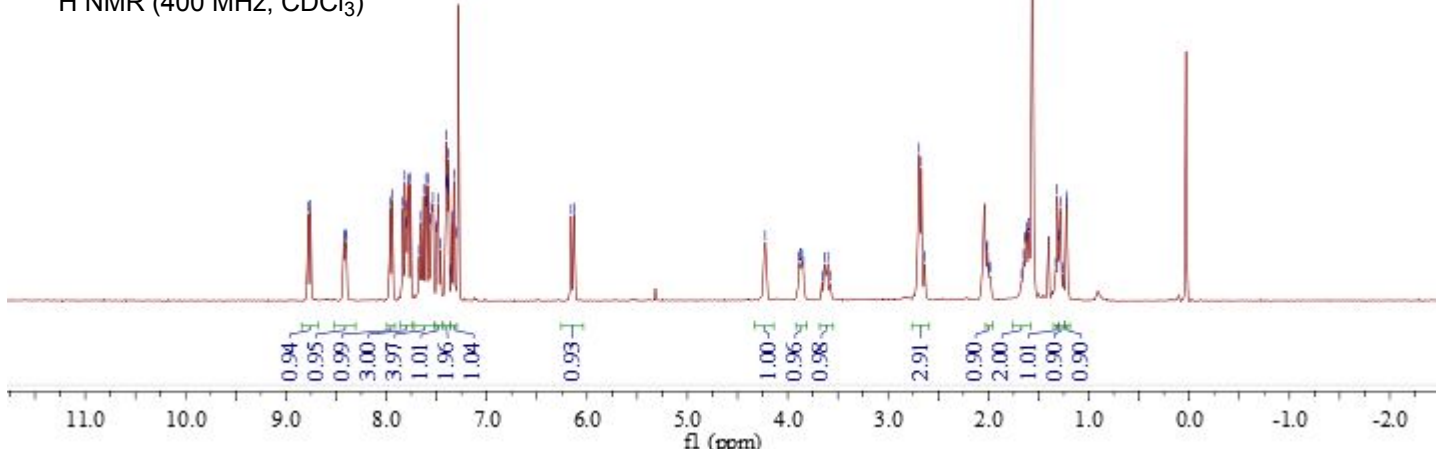

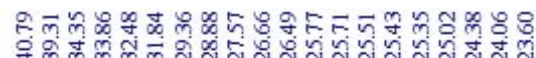

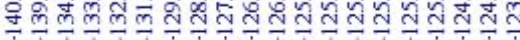

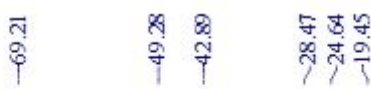

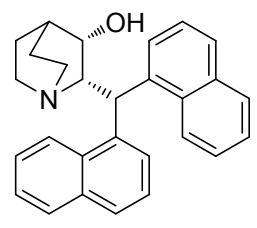

2w

${ }^{13} \mathrm{C}$ NMR $\left(151 \mathrm{MHz}, \mathrm{CDCl}_{3}\right)$

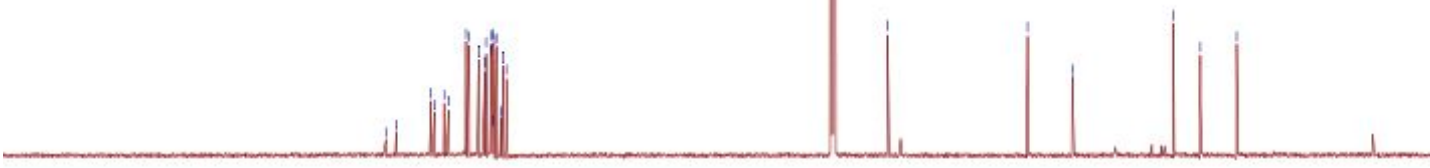

$\begin{array}{lllllllllll}190 & 180 & 170 & 160 & 150 & 140 & 130 & 120 & 110 & 100 & 90 \\ \mathrm{fl}(\mathrm{pom})\end{array}$ 
9. HPLC chromatograms for $2 a-2 w$

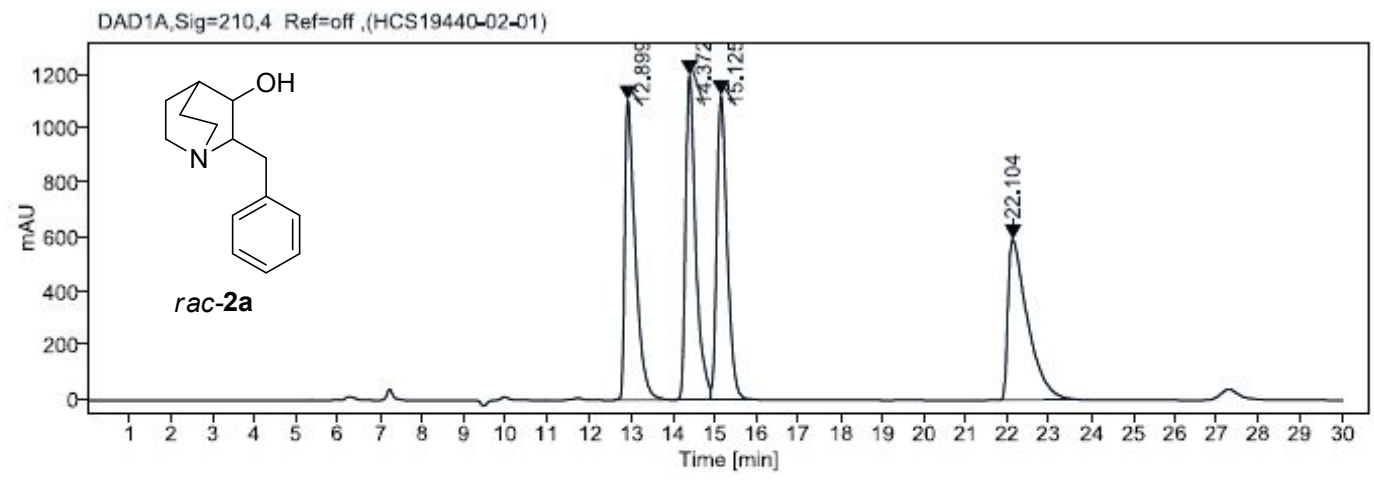

Signal: $\quad$ DAD1A,Sig $=210,4$ Ref=off

$\begin{array}{ccccccc}\text { RT [min] } & \text { Height } & \text { Area } & \text { Area \% } & \begin{array}{c}\text { Resolution } \\ \text { USP }\end{array} & \text { Tail } & \begin{array}{c}\text { Peak Theoretical } \\ \text { Plates USP }\end{array} \\ 12.90 & 1104.959 & 19353.173 & 24.86 & & 2.2 & 13082.47569 \\ 14,37 & 1195,718 & 20050,463 & 25,76 & 3,4 & 1.7 & 18735,19296 \\ 15.13 & 1120.360 & 19185.628 & 24.64 & 1.7 & 1.4 & 18314.81549 \\ 22,10 & 591,358 & 19260,340 & 24,74 & 10,9 & 2,8 & 11159,70748\end{array}$

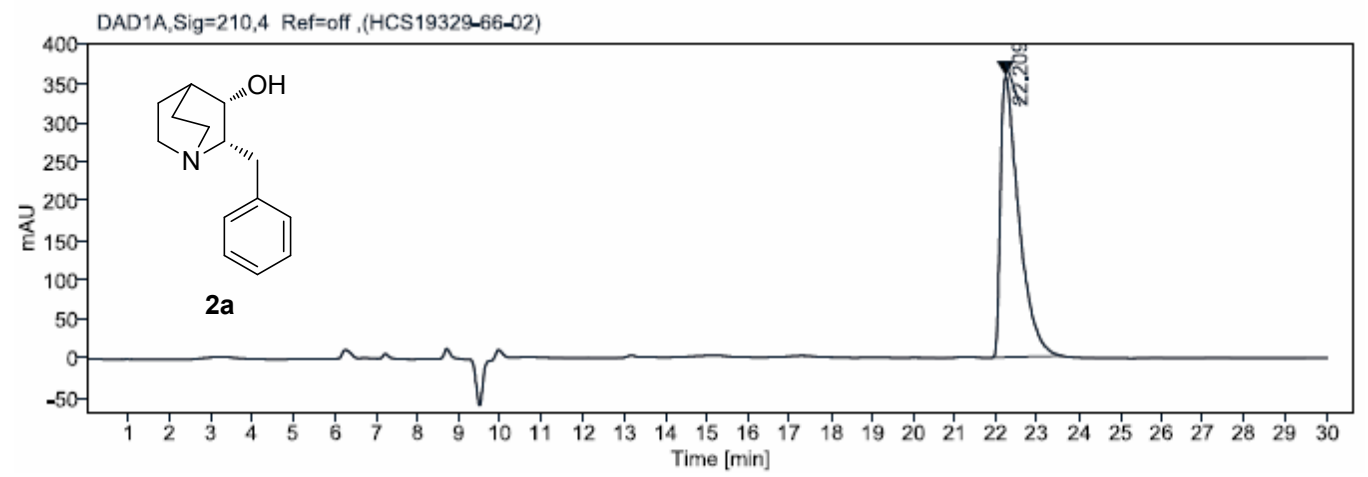

Signal: $\quad D A D 1 A, S i g=210,4$ Ref $=$ off

$\begin{array}{ccccccc}\text { RT [min] } & \text { Height } & \text { Area } & \text { Area \% } & \begin{array}{c}\text { Resolution } \\ \text { USP }\end{array} & \text { Tail } & \begin{array}{c}\text { Peak Theoretical } \\ \text { Plates USP }\end{array} \\ 22.21 & 357.764 & 11011.411 & 100.00 & & 2.5 & 12504.05993\end{array}$




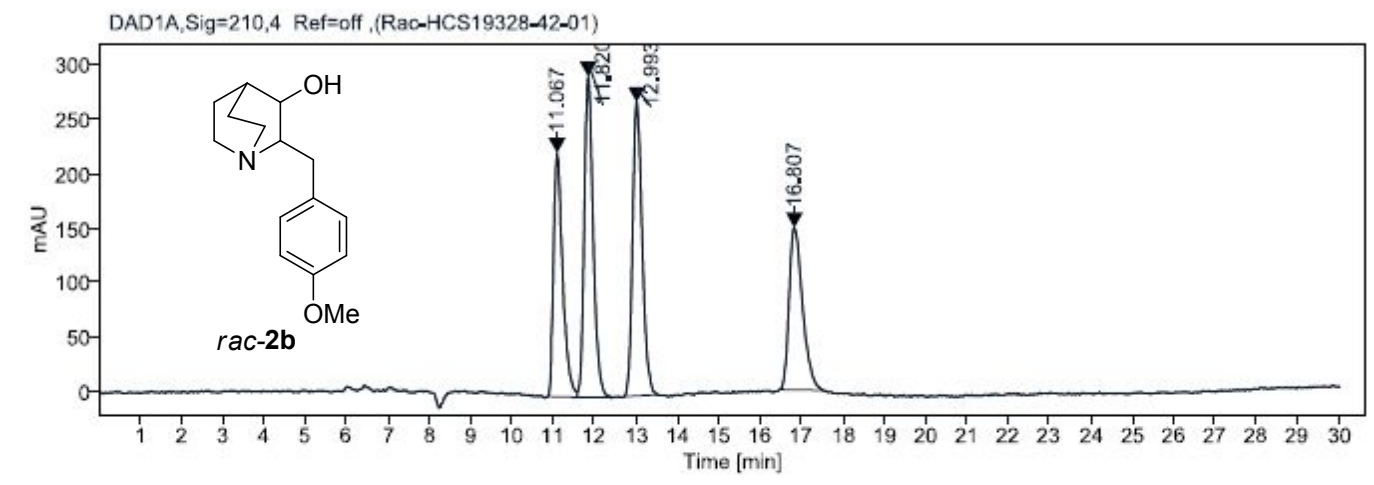

Signal: DAD1A,Sig $=210,4$ Ref $=$ off

$\begin{array}{ccccccc}\text { RT [min] } & \text { Height } & \text { Area } & \text { Area \% } & \begin{array}{c}\text { Resolution } \\ \text { USP }\end{array} & \text { Tail } & \begin{array}{c}\text { Peak Theoretical } \\ \text { Plates USP }\end{array} \\ 11.07 & 224.055 & 3440.142 & 22.13 & & 1.6 & 12429.09744 \\ 11,82 & 294,689 & 4409,330 & 28,36 & 1,9 & 1,3 & 14682,14183 \\ 12.99 & 269.915 & 4339.271 & 27.91 & 2.9 & 1.3 & 15003.76218 \\ 16,81 & 148,611 & 3358,338 & 21,60 & 7,5 & 1,5 & 12668,77938\end{array}$

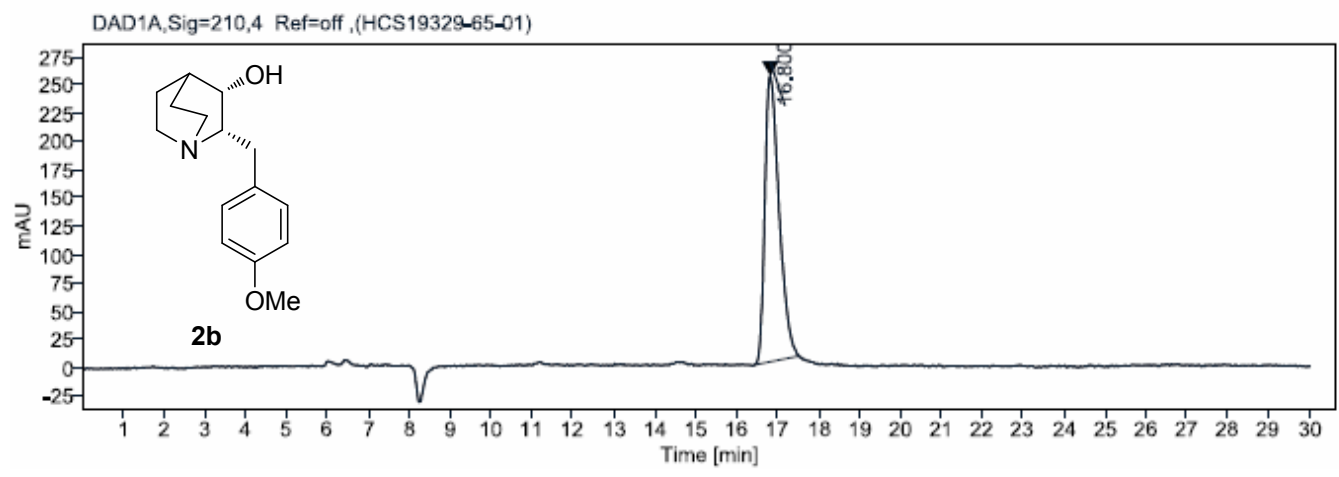

Signal: $\quad D A D 1 A, S i g=210,4$ Ref=off

$\begin{array}{ccccccc}\text { RT [min] } & \text { Height } & \text { Area } & \text { Area \% } & \begin{array}{c}\text { Resolution } \\ \text { USP }\end{array} & \text { Tail } & \begin{array}{c}\text { Peak Theoretical } \\ \text { Plates USP }\end{array} \\ 16.80 & 252.084 & 6100.089 & 100.00 & & 1.6 & 10895.23027\end{array}$




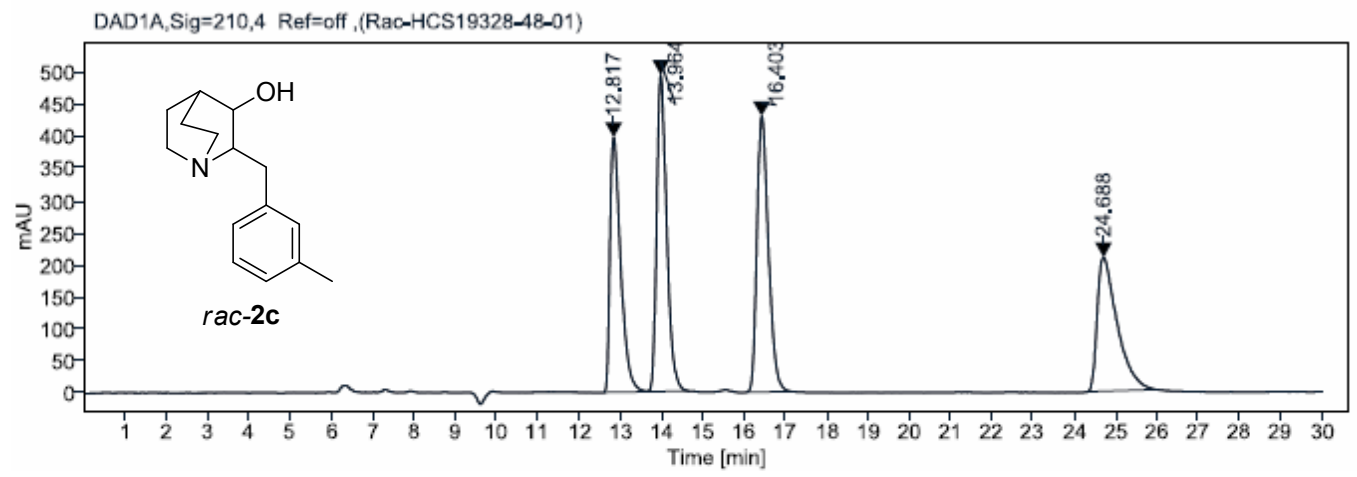

Signal: DAD1A,Sig $=210,4$ Ref $=$ off

$\begin{array}{ccccccc}\text { RT [min] } & \text { Height } & \text { Area } & \text { Area \% } & \begin{array}{c}\text { Resolution } \\ \text { USP }\end{array} & \text { Tail } & \begin{array}{c}\text { Peak Theoretical } \\ \text { Plates USP }\end{array} \\ 12.82 & 399.106 & 7147.826 & 23.13 & & 1.8 & 12110.12907 \\ 13,96 & 495,030 & 8342,914 & 27,00 & 2,5 & 1,4 & 16229,41233 \\ 16.40 & 431.360 & 8337.934 & 26.99 & 5.2 & 1.3 & 16592.53404 \\ 24,69 & 209,273 & 7067,589 & 22,88 & 12,0 & 2,2 & 12720,36450\end{array}$

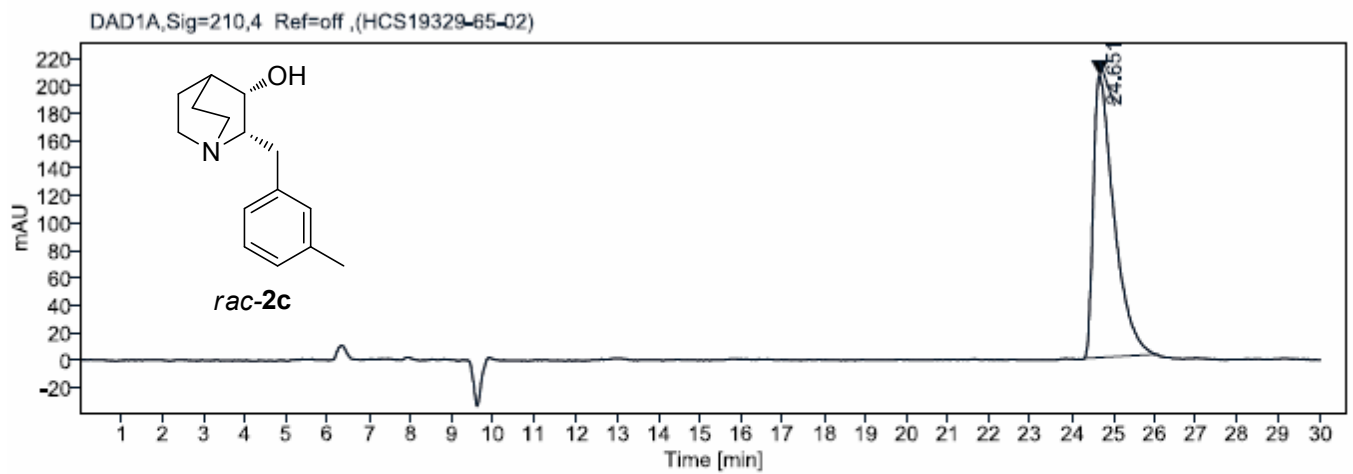

Signal: $\quad D A D 1 A, S i g=210,4$ Ref $=$ off

$\begin{array}{ccccccc}\text { RT [min] } & \text { Height } & \text { Area } & \text { Area \% } & \begin{array}{c}\text { Resolution } \\ \text { USP }\end{array} & \text { Tail } & \begin{array}{c}\text { Peak Theoretical } \\ \text { Plates USP }\end{array} \\ 24.65 & 206.729 & 7202.103 & 100.00 & & 2.2 & 12029.64452\end{array}$




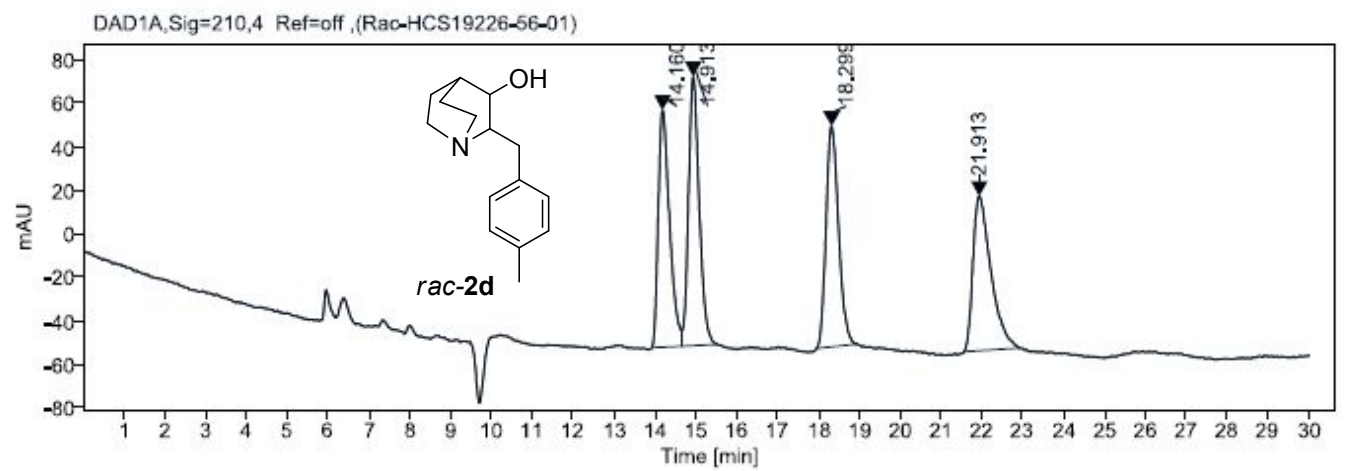

Signal: DAD1A,Sig $=210,4$ Ref $=$ off

$\begin{array}{ccccccc}\text { RT [min] } & \text { Height } & \text { Area } & \text { Area \% } & \begin{array}{c}\text { Resolution } \\ \text { USP }\end{array} & \text { Tail } & \begin{array}{c}\text { Peak Theoretical } \\ \text { Plates USP }\end{array} \\ 14.16 & 109.028 & 2102.067 & 24.18 & & 1.6 & 12511.18000 \\ 14,91 & 123,939 & 2256,770 & 25,96 & 1,5 & 1,2 & 15591,87212 \\ 18.30 & 101.478 & 2158.765 & 24.84 & 6.5 & 1.3 & 16843.12187 \\ 21,91 & 71,130 & 2174,600 & 25,02 & 5,3 & 1,9 & 12078,89029\end{array}$

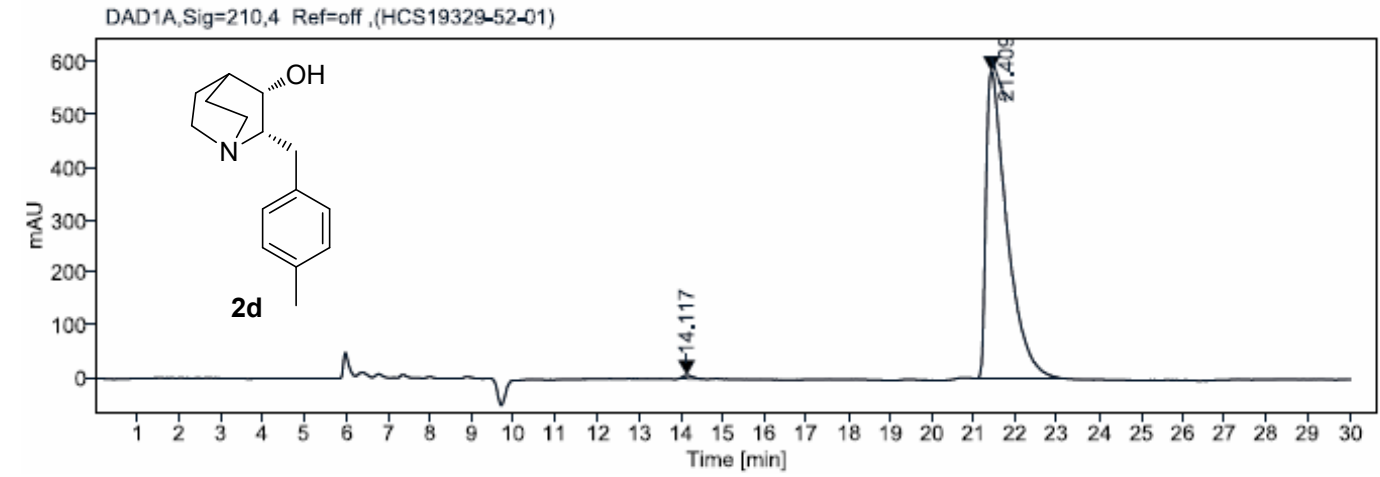

Signal: $\quad \mathrm{DAD} 1 \mathrm{~A}, \mathrm{Sig}=210,4$ Ref=off

$\begin{array}{ccccccc}\text { RT [min] } & \text { Height } & \text { Area } & \text { Area \% } & \begin{array}{c}\text { Resolution } \\ \text { USP }\end{array} & \text { Tail } & \begin{array}{c}\text { Peak Theoretical } \\ \text { Plates USP }\end{array} \\ 14.12 & 6.311 & 103.290 & 0.50 & & 1.3 & 15753.34685 \\ 21,41 & 584,465 & 20361,276 & 99,50 & 10,8 & 2,6 & 9159,21959\end{array}$




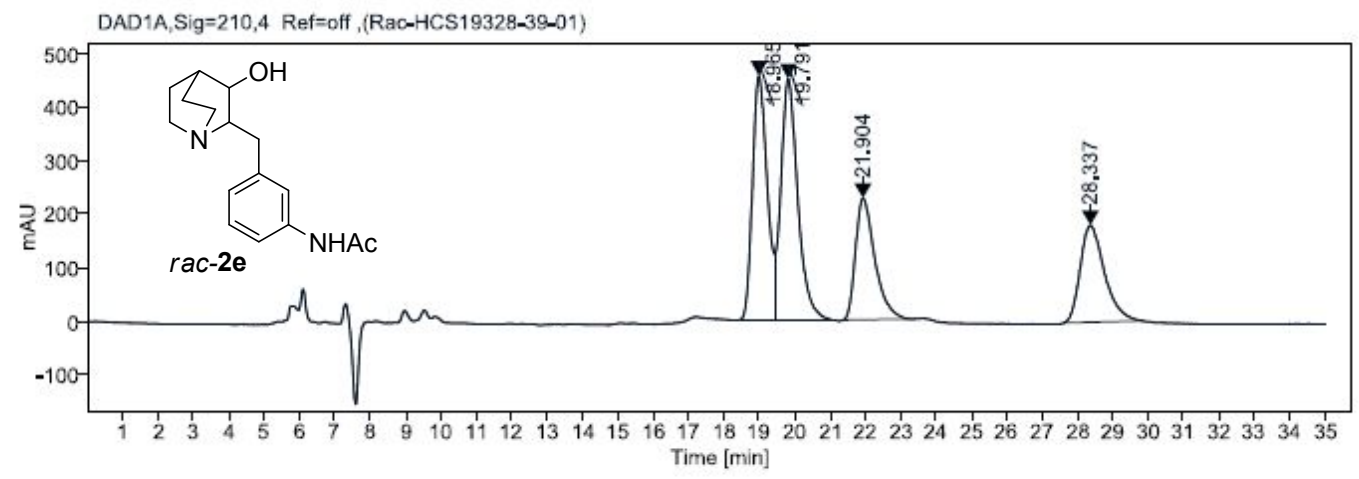

Signal: DAD1A,Sig $=210,4$ Ref $=$ off

$\begin{array}{ccccccc}\text { RT [min] } & \text { Height } & \text { Area } & \text { Area \% } & \begin{array}{c}\text { Resolution } \\ \text { USP }\end{array} & \text { Tail } & \begin{array}{c}\text { Peak Theoretical } \\ \text { Plates USP }\end{array} \\ 18.96 & 454.848 & 13088.142 & 28.89 & & 1.1 & 9269.53525 \\ 19,79 & 449,242 & 14961,666 & 33,02 & 1,0 & 1,5 & 8206,37787 \\ 21.90 & 226.596 & 8619.275 & 19.02 & 2.3 & 1.6 & 7951.48417 \\ 28,34 & 180,802 & 8637,497 & 19,06 & 5,8 & 1,5 & 8255,48742\end{array}$

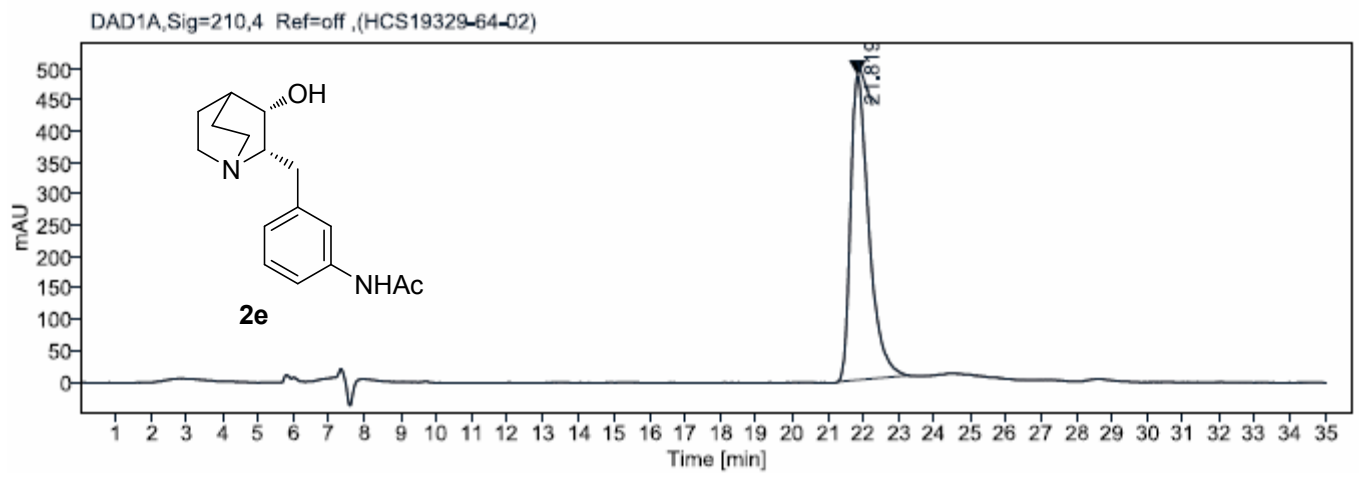

Signal: DAD1A,Sig=210,4 Ref=off

$\begin{array}{ccccccc}\text { RT [min] } & \text { Height } & \text { Area } & \text { Area \% } & \begin{array}{c}\text { Resolution } \\ \text { USP }\end{array} & \text { Tail } & \begin{array}{c}\text { Peak Theoretical } \\ \text { Plates USP }\end{array} \\ 21.82 & 486.968 & 17435.134 & 100.00 & & 1.7 & 9103.98471\end{array}$




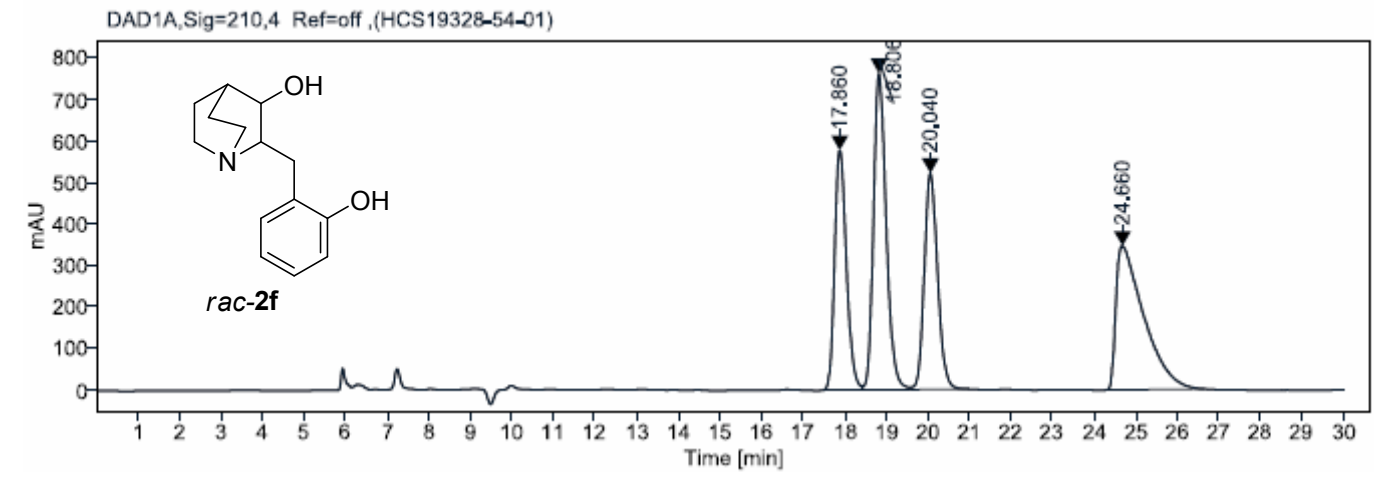

Signal: DAD1A,Sig=210,4 Ref=off

$\begin{array}{ccccccc}\text { RT [min] } & \text { Height } & \text { Area } & \text { Area \% } & \begin{array}{c}\text { Resolution } \\ \text { USP }\end{array} & \text { Tail } & \begin{array}{c}\text { Peak Theoretical } \\ \text { Plates USP }\end{array} \\ 17.86 & 576.580 & 11355.974 & 20.58 & & 1.3 & 18883.04685 \\ 18,81 & 761,479 & 16162,176 & 29,29 & 1,8 & 1,3 & 18451,88202 \\ 20.04 & 520.427 & 11535.384 & 20.90 & 2.2 & 1.2 & 18823.98772 \\ 24,66 & 346,125 & 16129,933 & 29,23 & 5,1 & 3,0 & 6405,25330\end{array}$

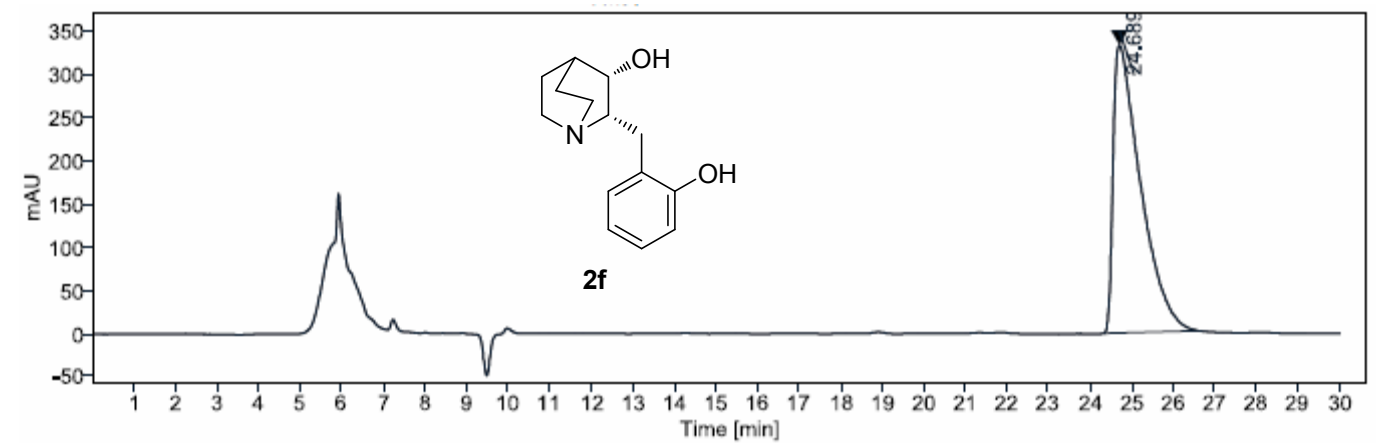

Signal: $\quad D A D 1 A, S i g=210,4$ Ref $=$ off

$\begin{array}{ccccccc}\text { RT [min] } & \text { Height } & \text { Area } & \text { Area \% } & \begin{array}{c}\text { Resolution } \\ \text { USP }\end{array} & \text { Tail } & \begin{array}{c}\text { Peak Theoretical } \\ \text { Plates USP }\end{array} \\ 24.69 & 332.069 & 15252.163 & 100.00 & & 3.0 & 6631.11696\end{array}$




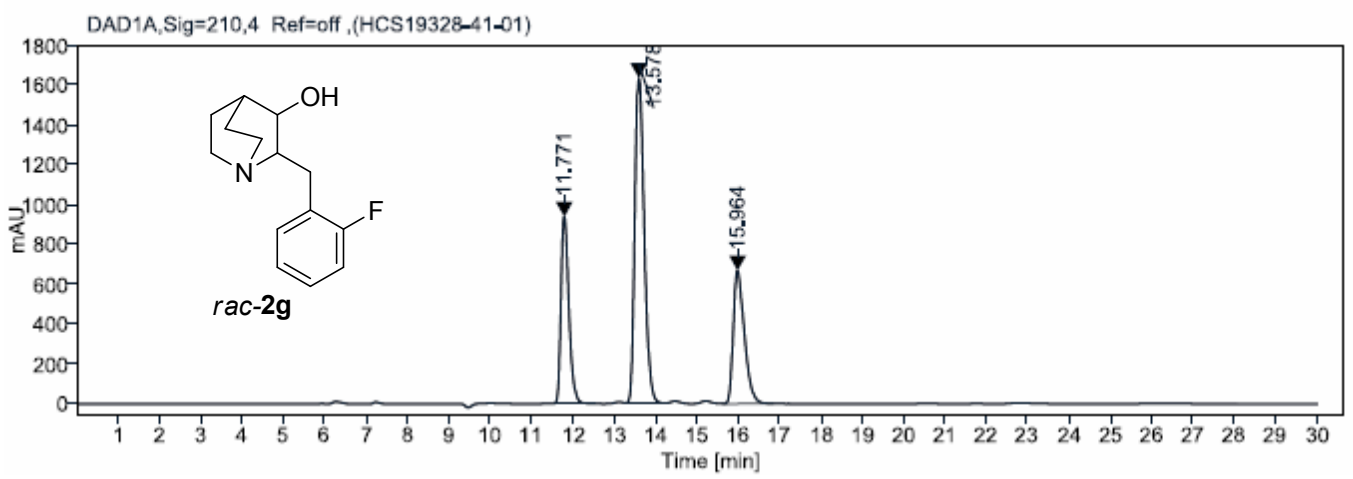

Signal: $\quad D A D 1 A, S i g=210,4$ Ref $=$ off

$\begin{array}{ccccccc}\text { RT [min] } & \text { Height } & \text { Area } & \text { Area \% } & \begin{array}{c}\text { Resolution } \\ \text { USP }\end{array} & \text { Tail } & \begin{array}{c}\text { Peak Theoretical } \\ \text { Plates USP }\end{array} \\ 11.77 & 938.189 & 12323.193 & 24.26 & & 1.4 & 19015.96215 \\ 13,58 & 1635,679 & 25947.719 & 51,09 & 4.8 & 1.3 & 17293.92479 \\ 15.96 & 669.198 & 12516.790 & 24.65 & 5.3 & 1.5 & 16698.48880\end{array}$

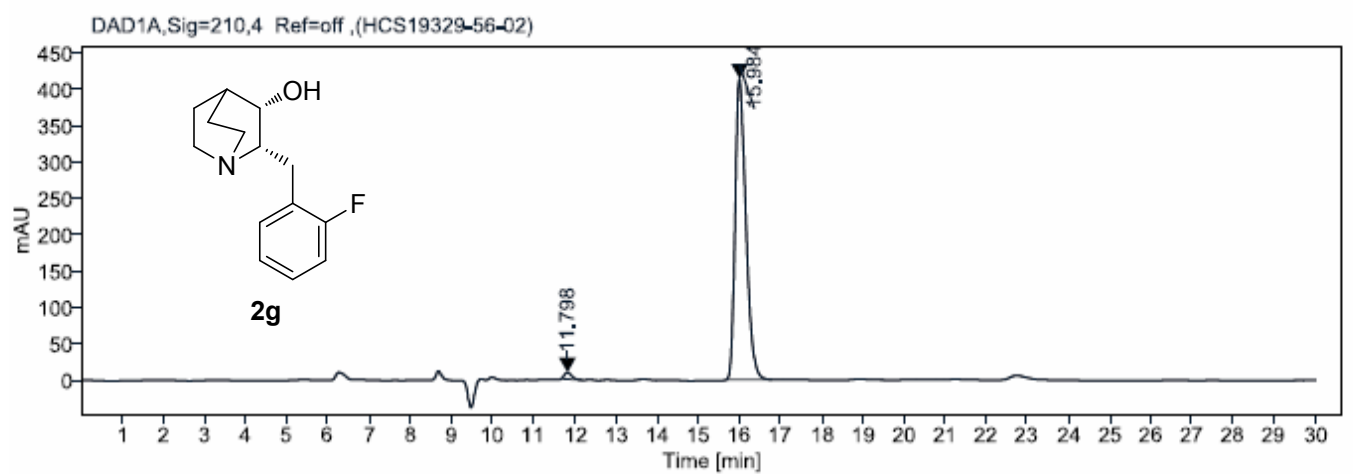

\begin{tabular}{ccccccc} 
Signal: & \multicolumn{2}{c}{ DAD1A,Sig=210,4 Ref=off } & & & & \\
RT [min] & Height & Area & Area $\%$ & $\begin{array}{c}\text { Resolution } \\
\text { USP }\end{array}$ & Tail & $\begin{array}{c}\text { Peak Theoretical } \\
\text { Plates USP }\end{array}$ \\
11.80 & 9.038 & 109.749 & 1.44 & & 1.1 & 21310.34608 \\
15,98 & 413,825 & 7522,501 & 98,56 & 10,4 & 1.4 & 17701,61412
\end{tabular}




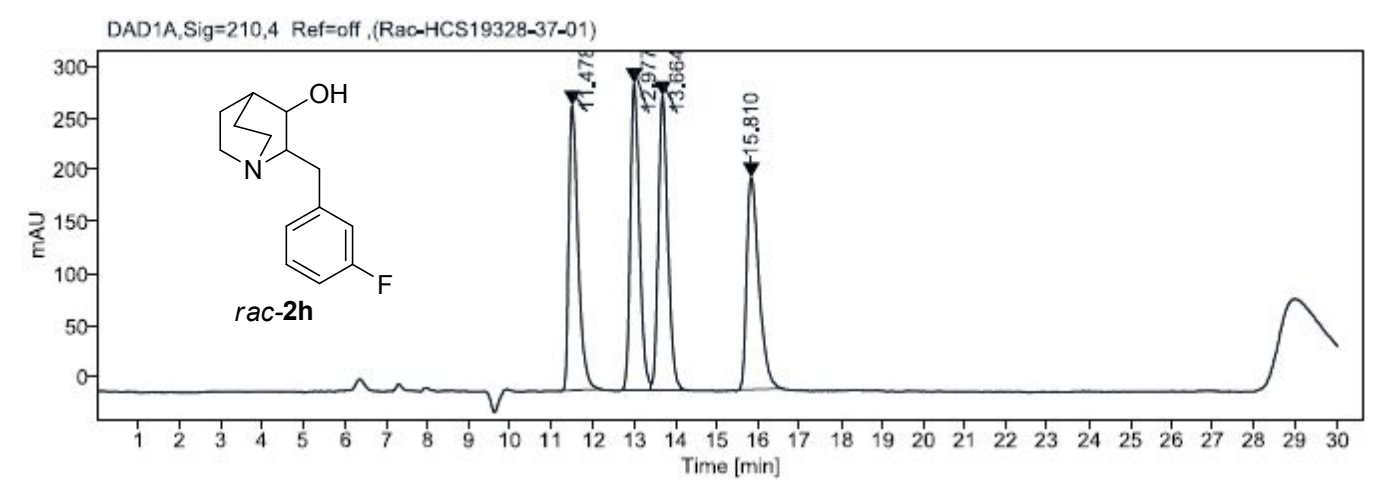

Signal: $\quad$ DAD1A,Sig=210,4 Ref=off

$\begin{array}{ccccccc}\text { RT [min] } & \text { Height } & \text { Area } & \text { Area \% } & \begin{array}{c}\text { Resolution } \\ \text { USP }\end{array} & \text { Tail } & \begin{array}{c}\text { Peak Theoretical } \\ \text { Plates USP }\end{array} \\ 11.48 & 274.847 & 4253.278 & 24.28 & & 1.6 & 13153.74297 \\ 12,98 & 295,872 & 4508,674 & 25,74 & 3,7 & 1,3 & 16791,43080 \\ 13.66 & 283.313 & 4551.280 & 25.98 & 1.7 & 1.2 & 16880.53312 \\ 15,81 & 204,557 & 4204,197 & 24,00 & 4,5 & 1,6 & 13843,92432\end{array}$

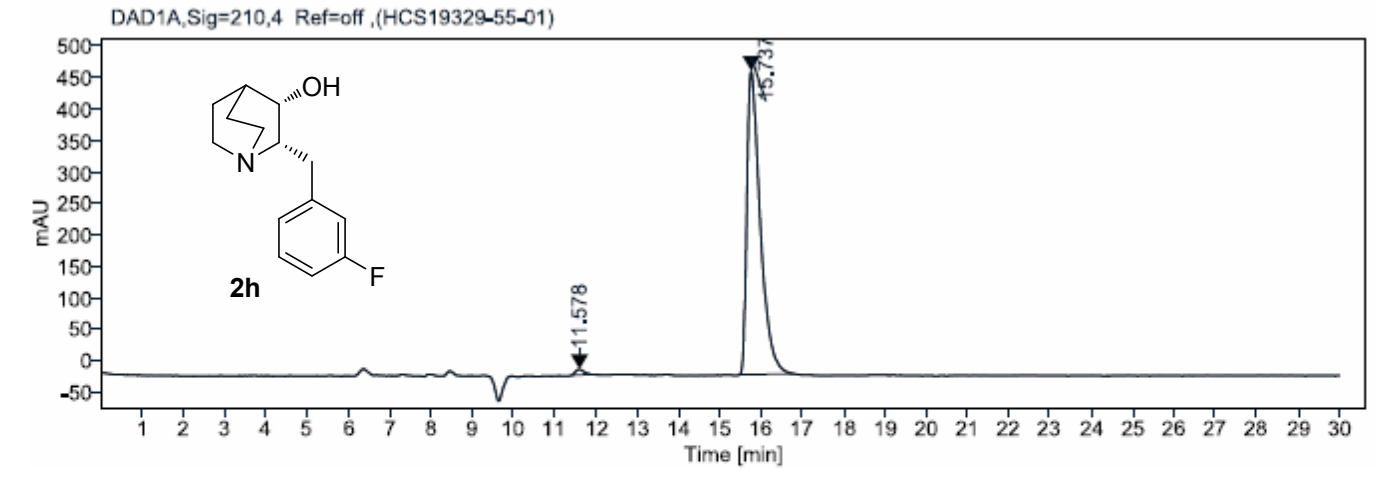

Signal: DAD1A,Sig $=210,4$ Ref $=$ off

$\begin{array}{ccccccc}\text { RT [min] } & \text { Height } & \text { Area } & \text { Area \% } & \begin{array}{c}\text { Resolution } \\ \text { USP }\end{array} & \text { Tail } & \begin{array}{c}\text { Peak Theoretical } \\ \text { Plates USP }\end{array} \\ 11.58 & 8.913 & 123.885 & 1.13 & & 1.5 & 15019.20486 \\ 15,74 & 482,344 & 10835,382 & 98,87 & 8,7 & 2,0 & 11710,77406\end{array}$




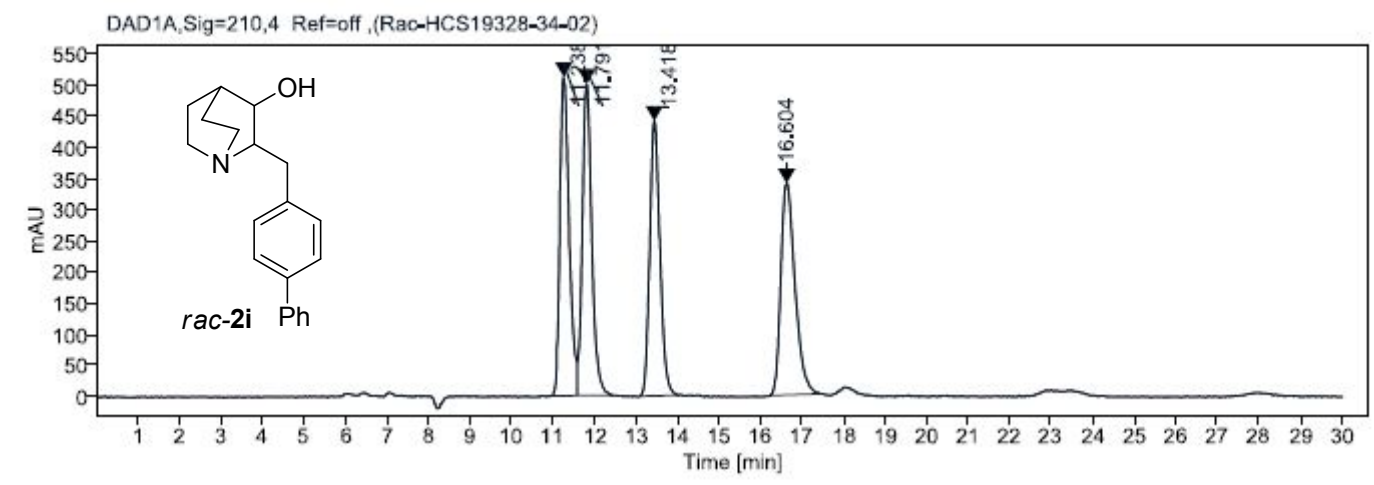

Signal: $\quad$ DAD1A,Sig $=210,4$ Ref $=$ off

$\begin{array}{ccccccc}\text { RT [min] } & \text { Height } & \text { Area } & \text { Area \% } & \begin{array}{c}\text { Resolution } \\ \text { USP }\end{array} & \text { Tail } & \begin{array}{c}\text { Peak Theoretical } \\ \text { Plates USP }\end{array} \\ 11.24 & 511.979 & 7894.642 & 25.07 & & 1.4 & 12274.93904 \\ 11,79 & 500,798 & 7907,672 & 25,12 & 1,4 & 1.2 & 13536,24252 \\ 13.42 & 442.093 & 7585.652 & 24.09 & 3.8 & 1.2 & 14253.04125 \\ 16,60 & 340,391 & 8097,765 & 25,72 & 6,0 & 1,4 & 11436,51972\end{array}$

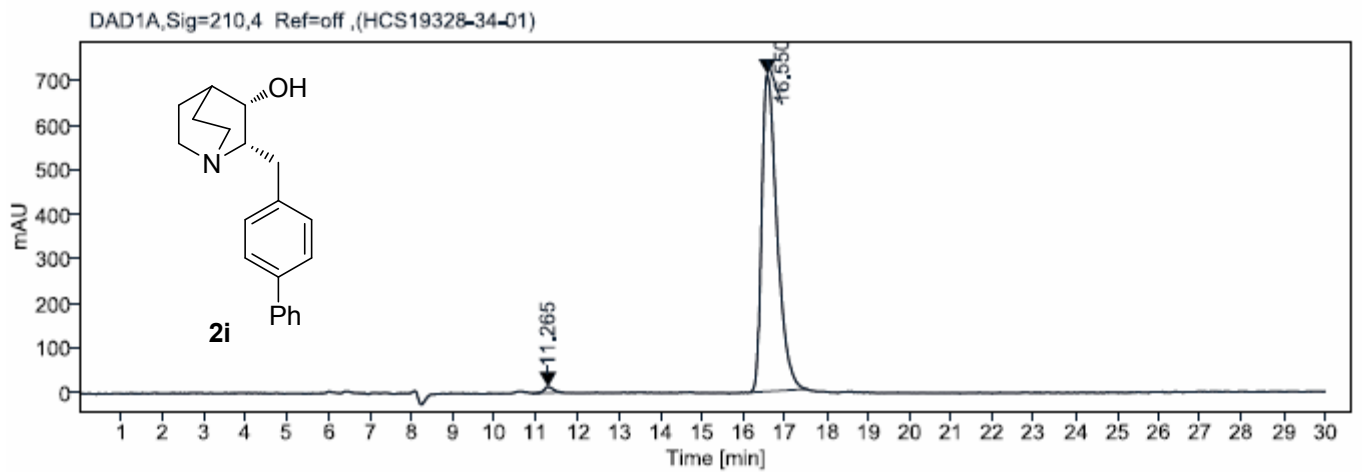

Signal: $\quad$ DAD1A,Sig $=210,4$ Ref $=$ off

$\begin{array}{ccccccc}\text { RT [min] } & \text { Height } & \text { Area } & \text { Area \% } & \begin{array}{c}\text { Resolution } \\ \text { USP }\end{array} & \text { Tail } & \begin{array}{c}\text { Peak Theoretical } \\ \text { Plates USP }\end{array} \\ 11.26 & 13.540 & 216.591 & 1.17 & & 1.4 & 11787.94247 \\ 16,55 & 710,872 & 18218,897 & 98,83 & 9,8 & 1,6 & 9919,74936\end{array}$




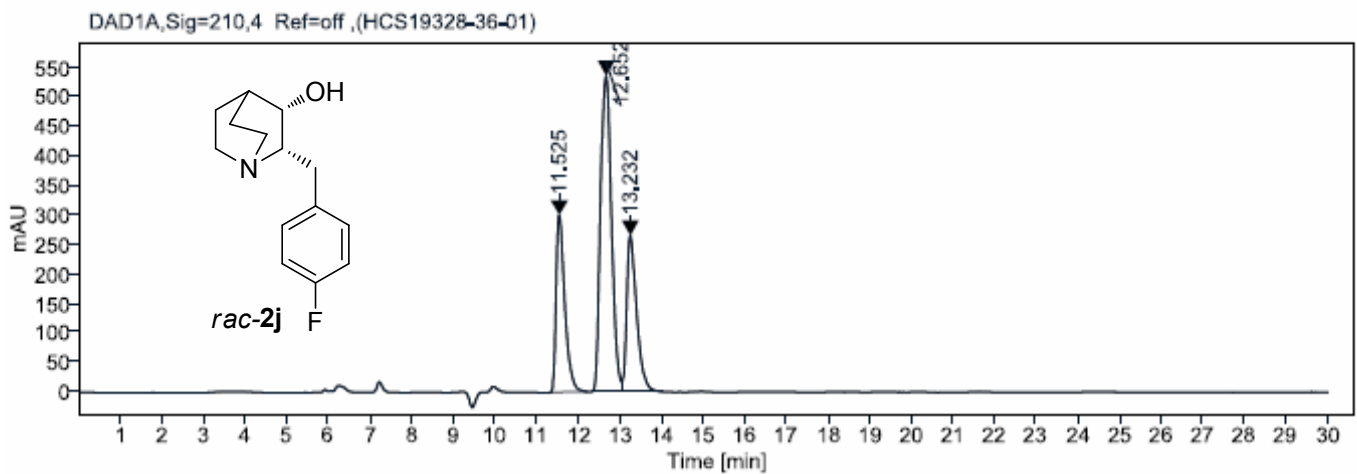

Signal: $\quad$ DAD1A,Sig $=210,4$ Ref $=$ off

$\begin{array}{ccccccc}\text { RT [min] } & \text { Height } & \text { Area } & \text { Area \% } & \begin{array}{c}\text { Resolution } \\ \text { USP }\end{array} & \text { Tail } & \begin{array}{c}\text { Peak Theoretical } \\ \text { Plates USP }\end{array} \\ 11.53 & 299.508 & 4311.595 & 23.30 & & 1.7 & 15360.03664 \\ 12,65 & 534,855 & 9782,757 & 52,88 & 2,6 & 1,1 & 11098,62065 \\ 13.23 & 264.199 & 4407.242 & 23.82 & 1.3 & 1.5 & 15133.93537\end{array}$

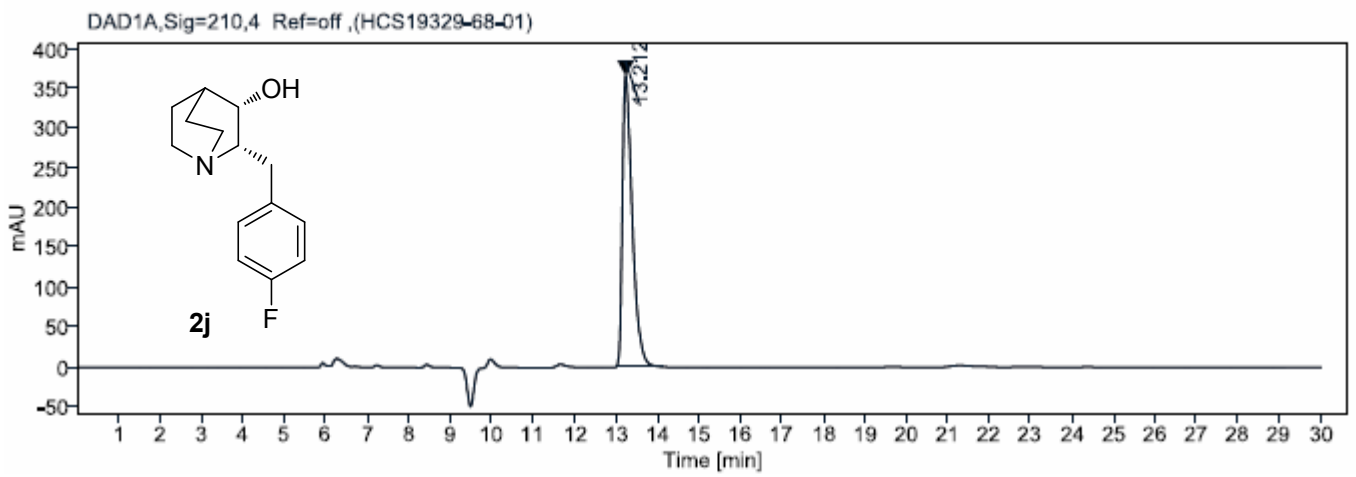

Signal: DAD1A,Sig=210,4 Ref=off

$\begin{array}{ccccccc}\text { RT [min] } & \text { Height } & \text { Area } & \text { Area \% } & \begin{array}{c}\text { Resolution } \\ \text { USP }\end{array} & \text { Tail } & \begin{array}{c}\text { Peak Theoretical } \\ \text { Plates USP }\end{array} \\ 13.21 & 366.053 & 6062.960 & 100.00 & & 1.8 & 15300.94727\end{array}$




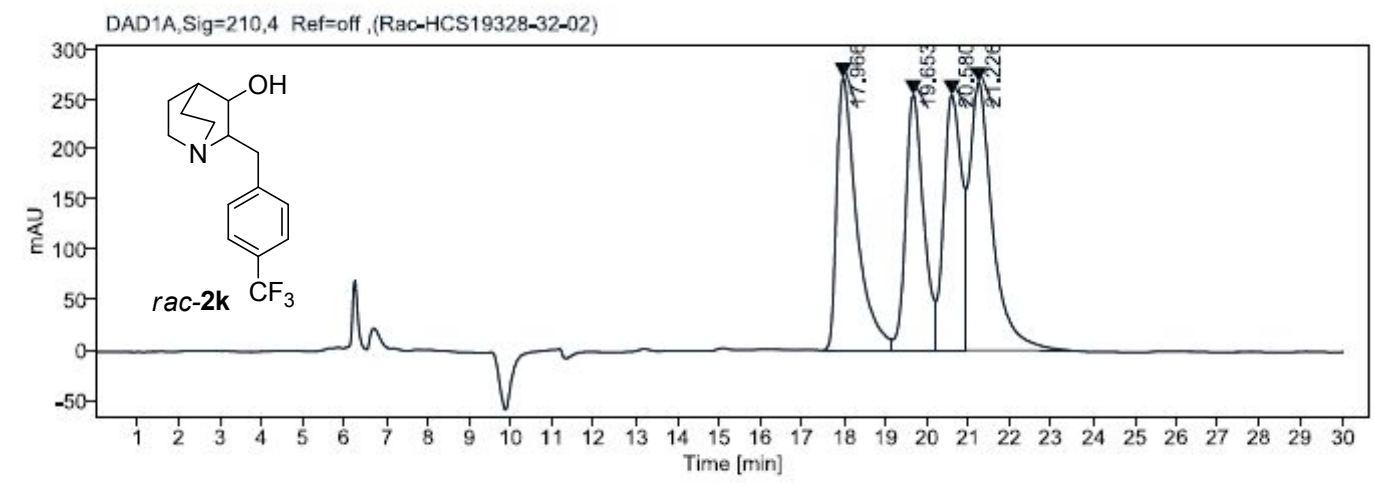

Signal: $\quad$ DAD1A, Sig $=210,4$ Ref $=$ off

$\begin{array}{ccccccc}\text { RT [min] } & \text { Height } & \text { Area } & \text { Area \% } & \begin{array}{c}\text { Resolution } \\ \text { USP }\end{array} & \text { Tail } & \begin{array}{c}\text { Peak Theoretical } \\ \text { Plates USP }\end{array} \\ 17.97 & 270.558 & 9082.080 & 26.25 & & 2.1 & 7589.79662 \\ 19,65 & 253,765 & 7605,793 & 21,98 & 2,1 & 1,1 & 9885,88276 \\ 20.58 & 253.478 & 7543.641 & 21.80 & 1.0 & 0.9 & 6703.75831 \\ 21,23 & 266,443 & 10370,926 & 29,97 & 0,6 & 2,2 & 5333,81265\end{array}$

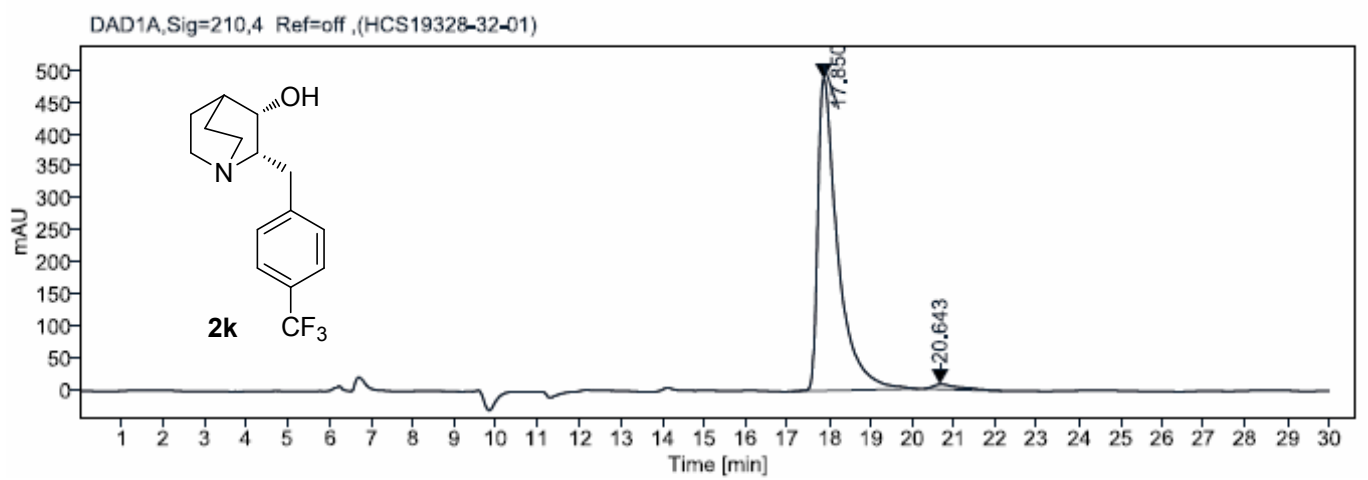

Signal: DAD1A,Sig $=210,4$ Ref $=$ off

$\begin{array}{ccccccc}\text { RT [min] } & \text { Height } & \text { Area } & \text { Area \% } & \begin{array}{c}\text { Resolution } \\ \text { USP }\end{array} & \text { Tail } & \begin{array}{c}\text { Peak Theoretical } \\ \text { Plates USP }\end{array} \\ 17.85 & 487.850 & 16253.720 & 97.88 & & 2.4 & 8241.94231 \\ 20,64 & 7,533 & 352,761 & 2,12 & 3,1 & 1,7 & 6790,70065\end{array}$




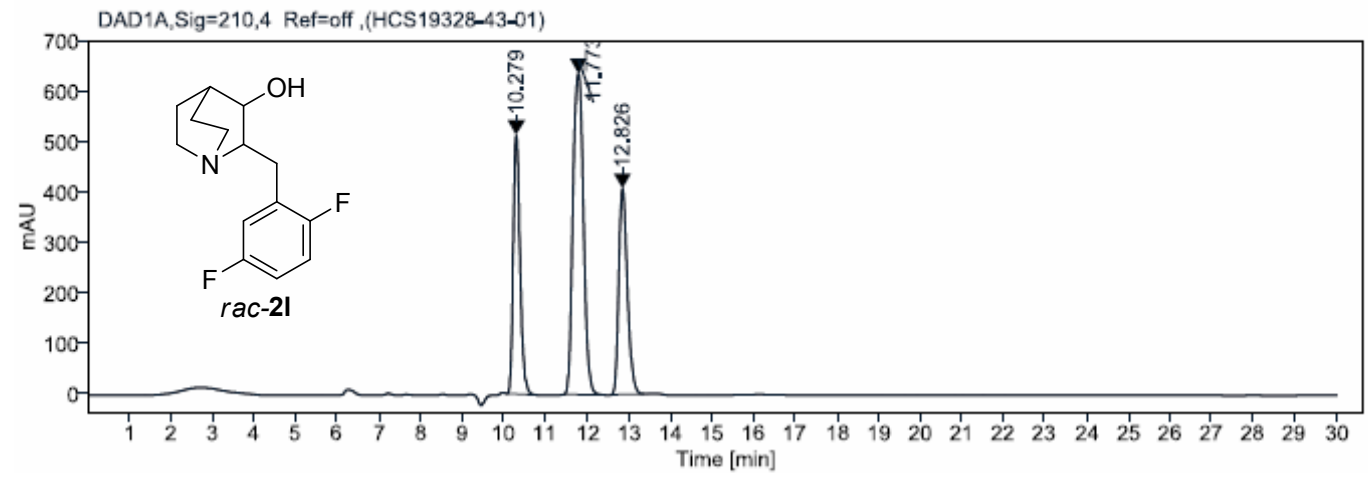

Signal: $\quad$ DAD1A,Sig $=210,4$ Ref $=$ off

$\begin{array}{ccccccc}\text { RT [min] } & \text { Height } & \text { Area } & \text { Area \% } & \begin{array}{c}\text { Resolution } \\ \text { USP }\end{array} & \text { Tail } & \begin{array}{c}\text { Peak Theoretical } \\ \text { Plates USP }\end{array} \\ 10.28 & 512.890 & 5738.238 & 25.25 & & 1.3 & 19912.03832 \\ 11.77 & 635.925 & 11156,862 & 49.10 & 4.0 & 1.1 & 10751,56348 \\ 12.83 & 408.525 & 5828.992 & 25.65 & 2.6 & 1.3 & 18610.56970\end{array}$

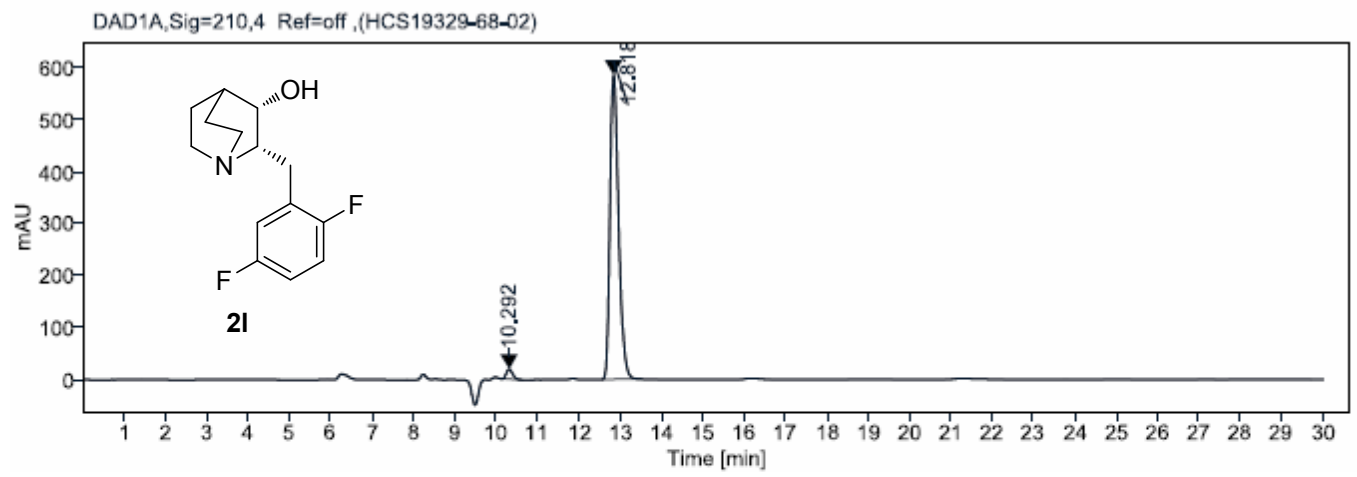

Signal: $\quad$ DAD1A,Sig $=210,4$ Ref $=$ off

$\begin{array}{ccccccc}\text { RT [min] } & \text { Height } & \text { Area } & \text { Area \% } & \begin{array}{c}\text { Resolution } \\ \text { USP }\end{array} & \text { Tail } & \begin{array}{c}\text { Peak Theoretical } \\ \text { Plates USP }\end{array} \\ 10.29 & 19.008 & 198.853 & 2.31 & & 1.1 & 21701.85203 \\ 12,82 & 586,103 & 8428,041 & 97,69 & 7.7 & 1.4 & 18362,80588\end{array}$




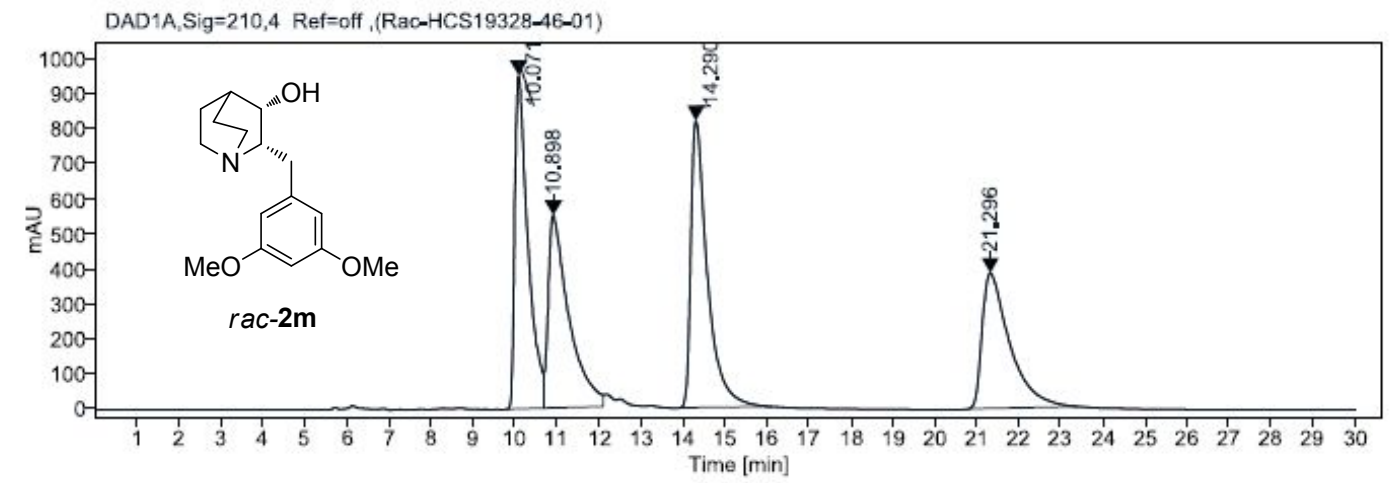

Signal: $\quad$ DAD1A,Sig $=210,4$ Ref $=$ off

$\begin{array}{ccccccc}\text { RT [min] } & \text { Height } & \text { Area } & \text { Area \% } & \begin{array}{c}\text { Resolution } \\ \text { USP }\end{array} & \text { Tail } & \begin{array}{c}\text { Peak Theoretical } \\ \text { Plates USP }\end{array} \\ 10.07 & 950.572 & 20706.303 & 25.97 & & 2.3 & 5308.23254 \\ 10,90 & 549,138 & 18881,125 & 23,68 & 1,2 & 3.1 & 2699,52813 \\ 14.29 & 817.911 & 22485.821 & 28.20 & 4.5 & 2.2 & 7413.36662 \\ 21,30 & 386,054 & 17655,947 & 22,14 & 7.8 & 2,4 & 5598,05933\end{array}$

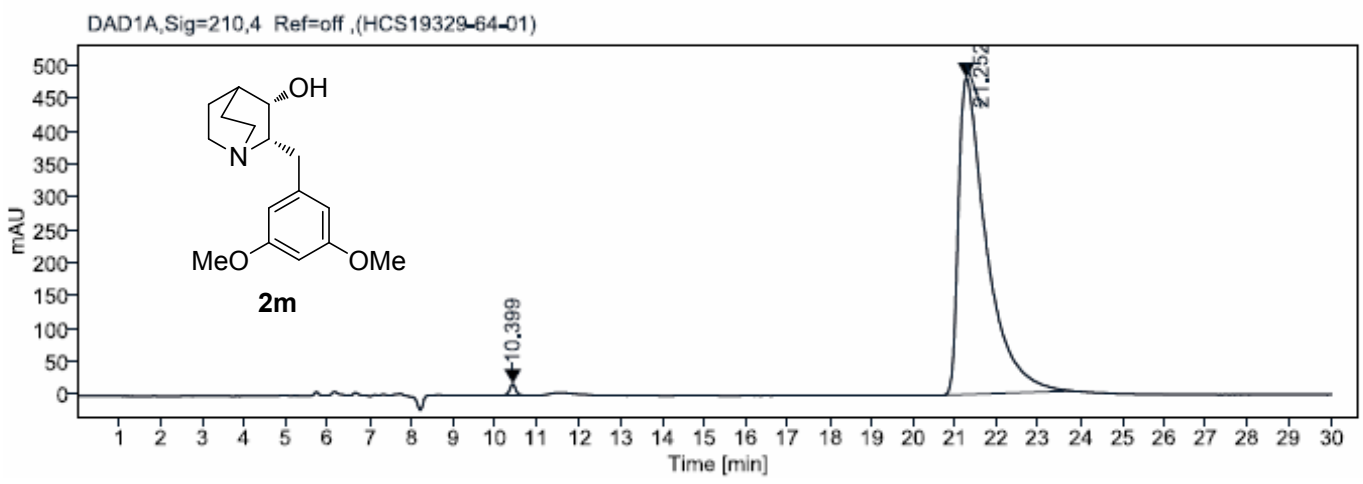

Signal: $\quad$ DAD1A, Sig $=210,4$ Ref $=$ off

$\begin{array}{ccccccc}\text { RT [min] } & \text { Height } & \text { Area } & \text { Area \% } & \begin{array}{c}\text { Resolution } \\ \text { USP }\end{array} & \text { Tail } & \begin{array}{c}\text { Peak Theoretical } \\ \text { Plates USP }\end{array} \\ 10.40 & 17.024 & 169.047 & 0.75 & & 1.0 & 24866.91848 \\ 21,25 & 480,511 & 22428,482 & 99,25 & 15.4 & 2,6 & 5530,01892\end{array}$




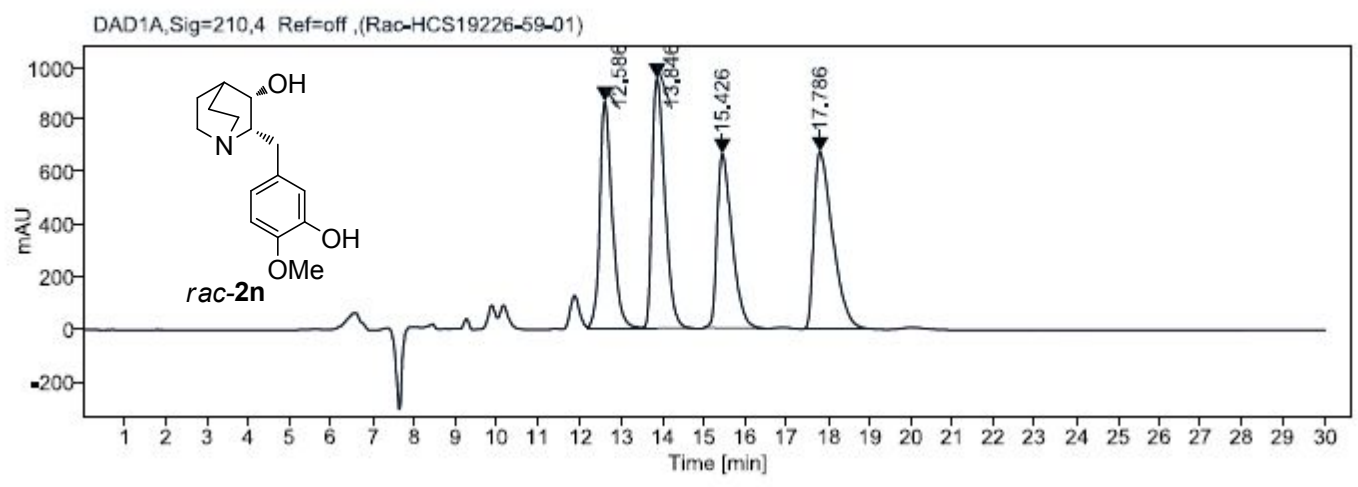

Signal: $\quad$ DAD1A,Sig $=210,4$ Ref $=$ off

\begin{tabular}{|c|c|c|c|c|c|c|}
\hline RT [min] & Height & Area & Area \% & $\begin{array}{l}\text { Resolution } \\
\text { USP }\end{array}$ & Tail & $\begin{array}{l}\text { Peak Theoretical } \\
\text { Plates USP }\end{array}$ \\
\hline 12.59 & 869.162 & 18146.249 & 23.69 & & 1.3 & 9000.07222 \\
\hline 13,85 & 956,970 & 20480,202 & 26,74 & 2,3 & 1,5 & 9650,92605 \\
\hline 15.43 & 666.767 & 16753.731 & 21.87 & 2.6 & 1.6 & 8621.47087 \\
\hline 17,79 & 677,298 & 21221,872 & 27,70 & 3,1 & 1,9 & 7052,89067 \\
\hline
\end{tabular}

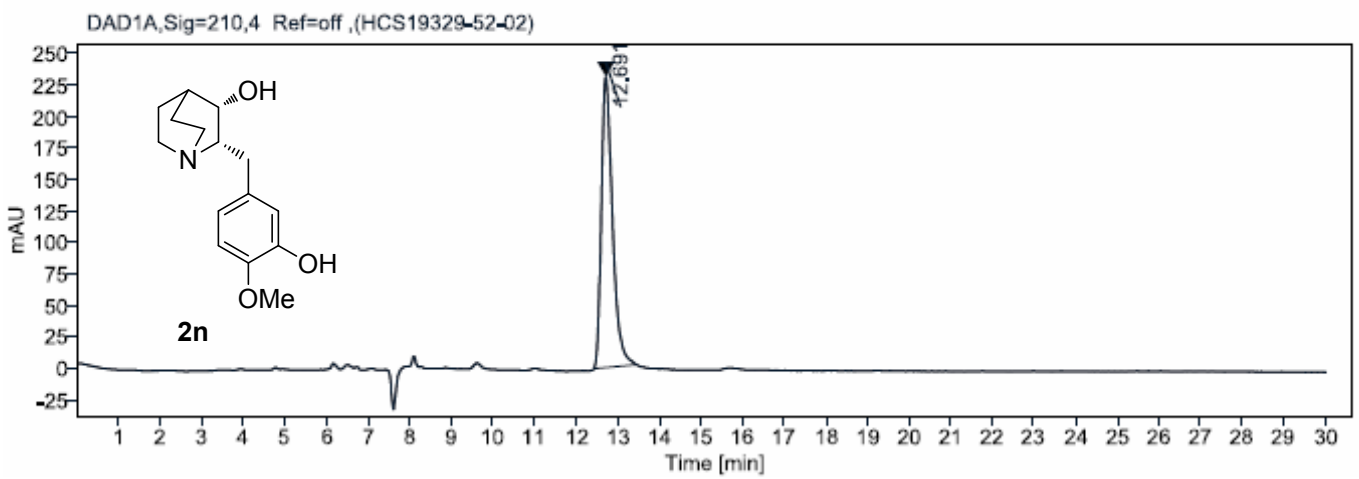

Signal: DAD1A,Sig=210,4 Ref=off

$\begin{array}{ccccccc}\text { RT [min] } & \text { Height } & \text { Area } & \text { Area \% } & \begin{array}{c}\text { Resolution } \\ \text { USP }\end{array} & \text { Tail } & \begin{array}{c}\text { Peak Theoretical } \\ \text { Plates USP }\end{array} \\ 12.69 & 231.509 & 4291.221 & 100.00 & & 1.6 & 11288.85176\end{array}$




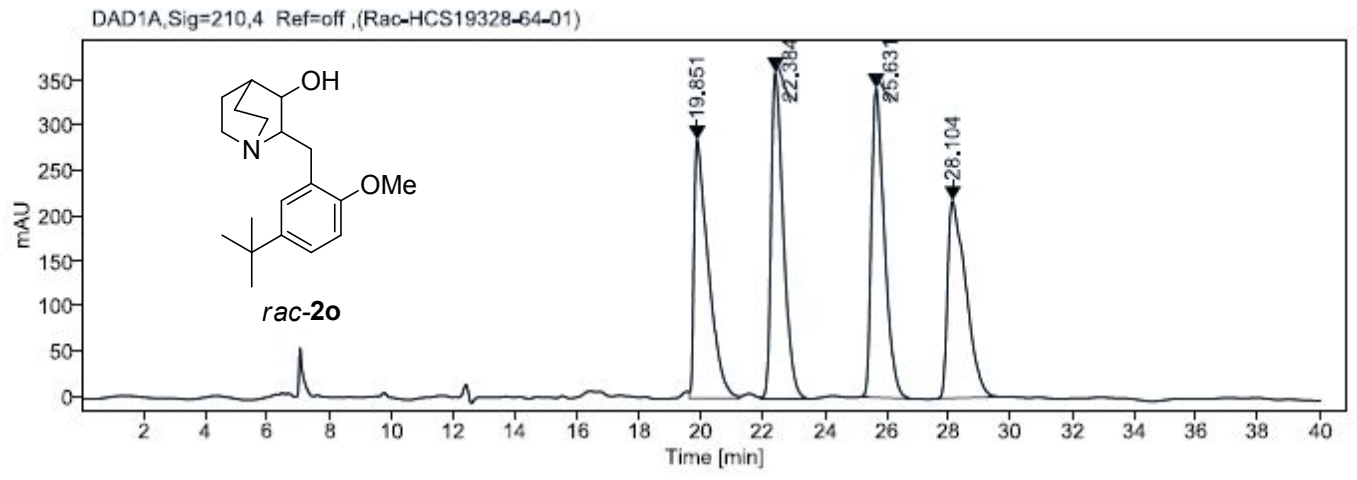

Signal: $\quad D A D 1 A, S i g=210,4$ Ref $=$ off

$\begin{array}{ccccccc}\text { RT [min] } & \text { Height } & \text { Area } & \text { Area \% } & \begin{array}{c}\text { Resolution } \\ \text { USP }\end{array} & \text { Tail } & \begin{array}{c}\text { Peak Theoretical } \\ \text { Plates USP }\end{array} \\ 19.85 & 286.206 & 9206.455 & 24.17 & & 3.0 & 9417.80793 \\ 22,38 & 361,768 & 9955,166 & 26,14 & 3,3 & 1,6 & 15146,89965 \\ 25.63 & 342.544 & 9952,680 & 26.13 & 4.3 & 1.6 & 17805.51788 \\ 28,10 & 218,596 & 8971,101 & 23,56 & 2,6 & 2,1 & 9725,84006\end{array}$

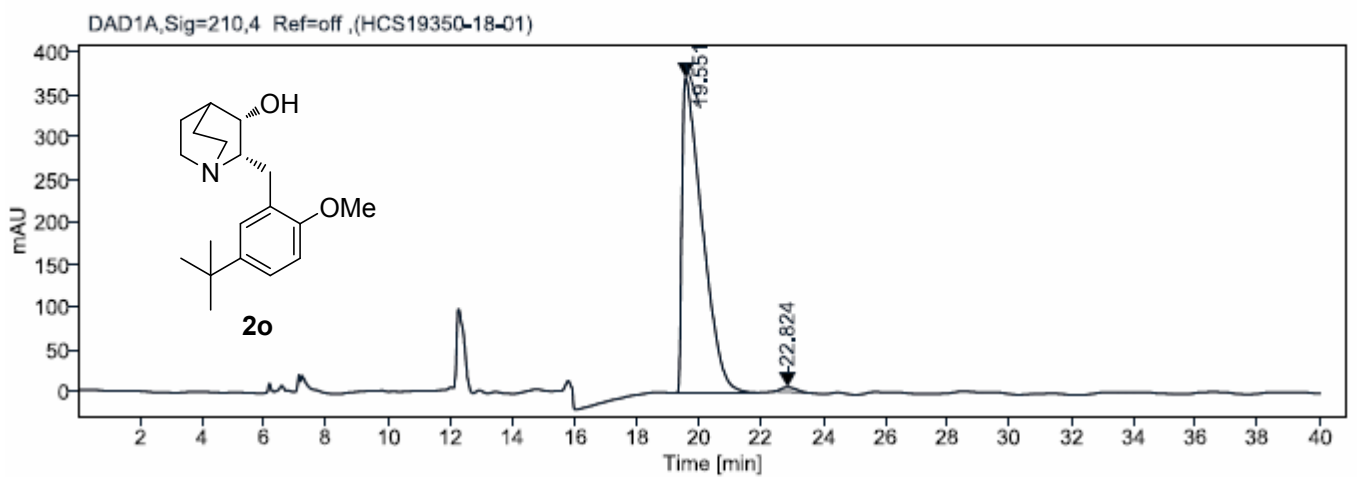

Signal: $\quad$ DAD1A,Sig=210,4 Ref $=$ off

$\begin{array}{ccccccc}\text { RT [min] } & \text { Height } & \text { Area } & \text { Area \% } & \begin{array}{c}\text { Resolution } \\ \text { USP }\end{array} & \text { Tail } & \begin{array}{c}\text { Peak Theoretical } \\ \text { Plates USP }\end{array} \\ 19.55 & 371.495 & 16814.530 & 98.84 & & 3.6 & 4693.11540 \\ 22,82 & 5,697 & 196,708 & 1,16 & 3.1 & 1.1 & 9427,22459\end{array}$




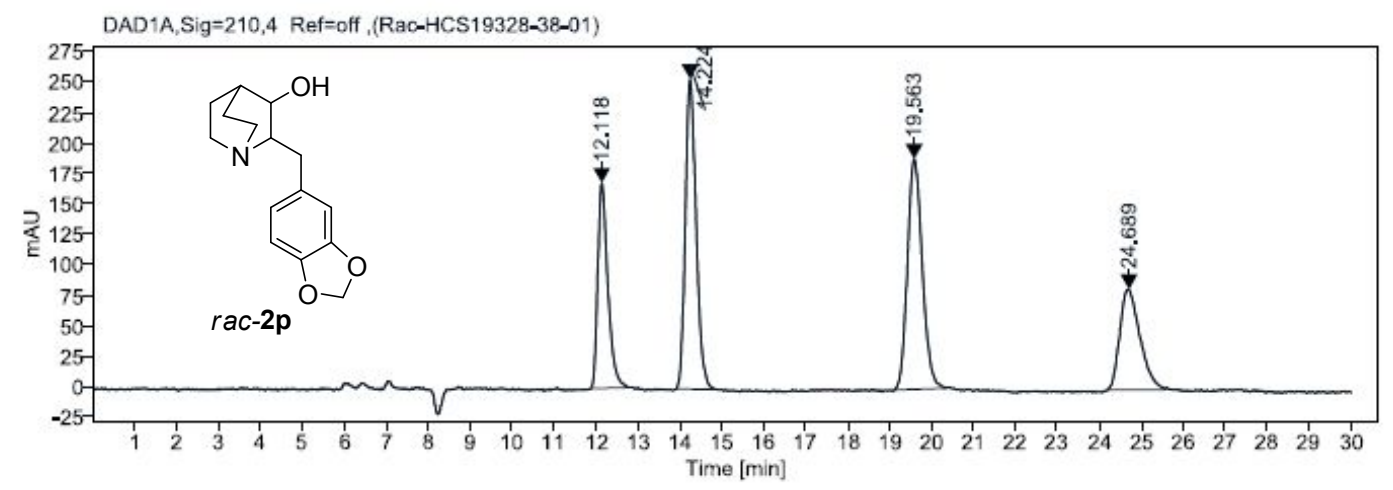

Signal: $\quad$ DAD1A,Sig $=210,4$ Ref $=$ off

$\begin{array}{ccccccc}\text { RT [min] } & \text { Height } & \text { Area } & \text { Area \% } & \begin{array}{c}\text { Resolution } \\ \text { USP }\end{array} & \text { Tail } & \begin{array}{c}\text { Peak Theoretical } \\ \text { Plates USP }\end{array} \\ 12.12 & 167.933 & 2811.367 & 19.10 & & 1.5 & 12638.85170 \\ 14,22 & 253,323 & 4529,751 & 30,77 & 4,7 & 1,3 & 14477,38030 \\ 19.56 & 188.485 & 4631.531 & 31.46 & 9.5 & 1.2 & 14539.52846 \\ 24,69 & 82,104 & 2749,216 & 18,67 & 6,6 & 1,4 & 12183,65696\end{array}$

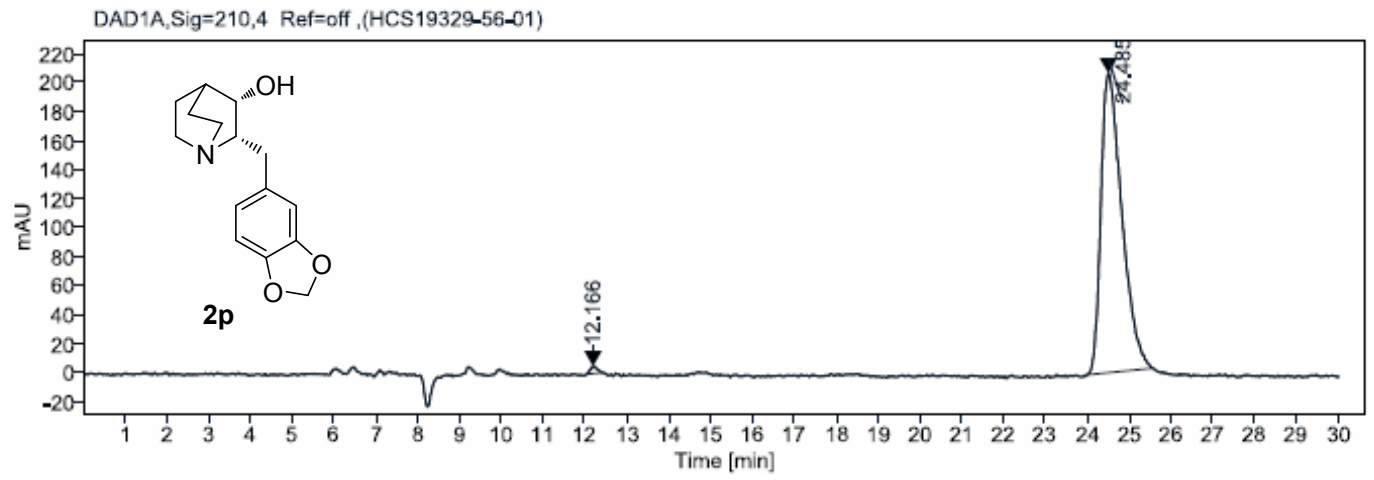

Signal: $\quad$ DAD1A,Sig $=210,4$ Ref $=$ off

$\begin{array}{ccccccc}\text { RT [min] } & \text { Height } & \text { Area } & \text { Area \% } & \begin{array}{c}\text { Resolution } \\ \text { USP }\end{array} & \text { Tail } & \begin{array}{c}\text { Peak Theoretical } \\ \text { Plates USP }\end{array} \\ 12.17 & 5.034 & 57.189 & 0.79 & & 1.5 & 24685.38373 \\ 24,49 & 206,770 & 7194,368 & 99,21 & 19,9 & 1.7 & 11047,65843\end{array}$




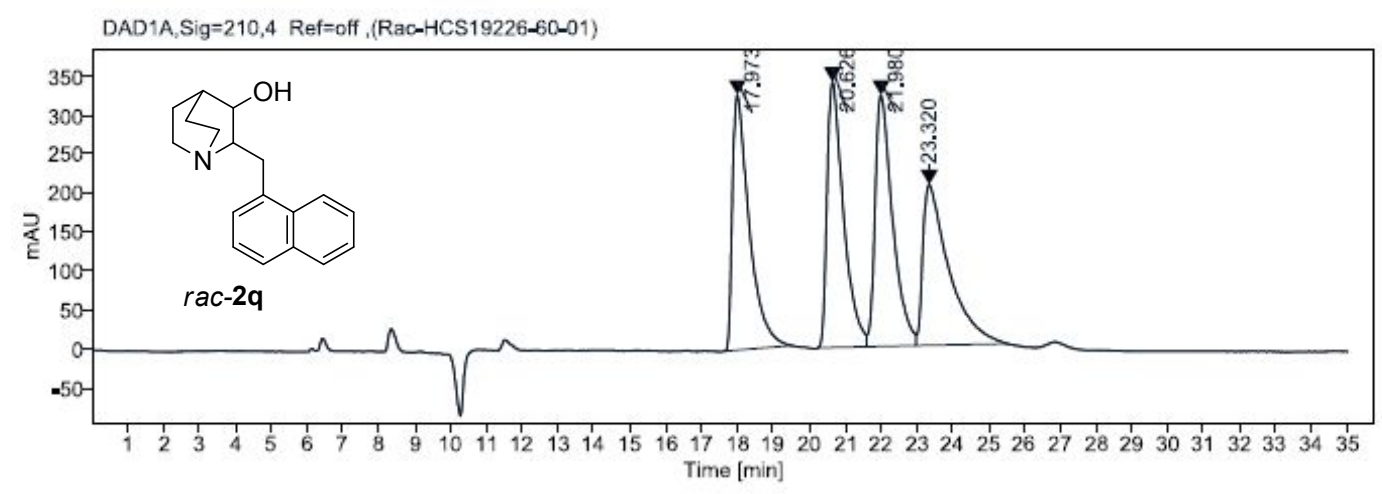

Signal: $\quad D A D 1 A, S i g=210,4$ Ref $=$ off

$\begin{array}{ccccccc}\text { RT [min] } & \text { Height } & \text { Area } & \text { Area \% } & \begin{array}{c}\text { Resolution } \\ \text { USP }\end{array} & \text { Tail } & \begin{array}{c}\text { Peak Theoretical } \\ \text { Plates USP }\end{array} \\ 17.97 & 327.507 & 10724.873 & 24.92 & & 2.6 & 7594.14682 \\ 20,63 & 340,834 & 10889,851 & 25,30 & 3,2 & 2,0 & 10039,00576 \\ 21.98 & 322.602 & 10993,960 & 25.54 & 1.6 & 1.8 & 9960.95724 \\ 23,32 & 206,478 & 10433,299 & 24,24 & 1,3 & 2,9 & 5768,07321\end{array}$

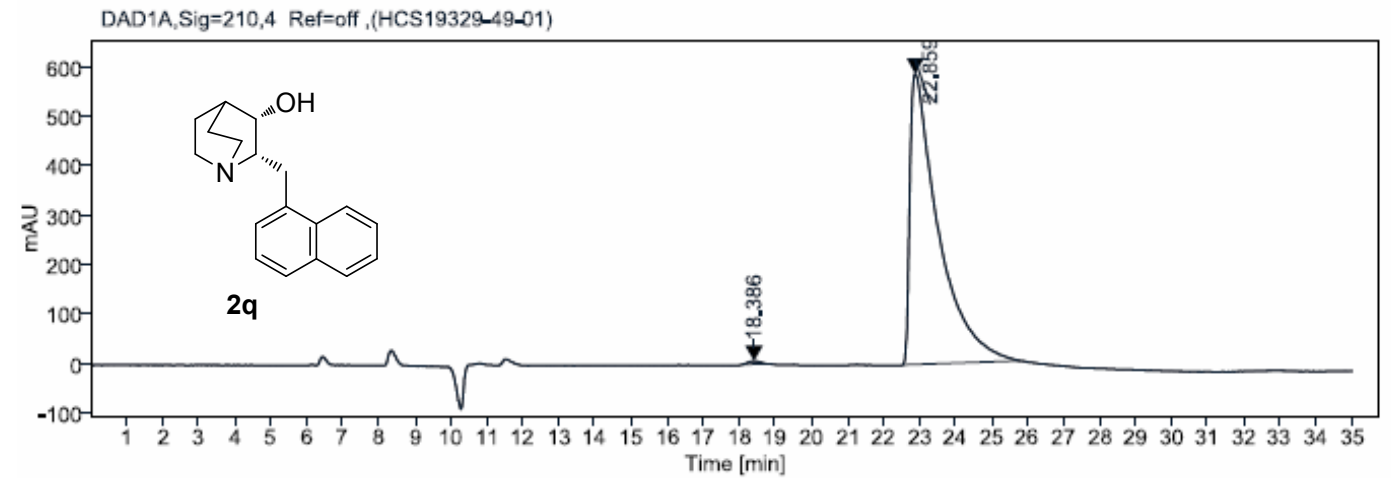

Signal: $\quad$ DAD1A,Sig=210,4 Ref=off

$\begin{array}{ccccccc}\text { RT [min] } & \text { Height } & \text { Area } & \text { Area \% } & \begin{array}{c}\text { Resolution } \\ \text { USP }\end{array} & \text { Tail } & \begin{array}{c}\text { Peak Theoretical } \\ \text { Plates USP }\end{array} \\ 18.39 & 6.017 & 131.180 & 0.41 & & 1.1 & 15388.45308 \\ 22,86 & 588,608 & 32247,293 & 99,59 & 4,9 & 4,5 & 5534,65414\end{array}$




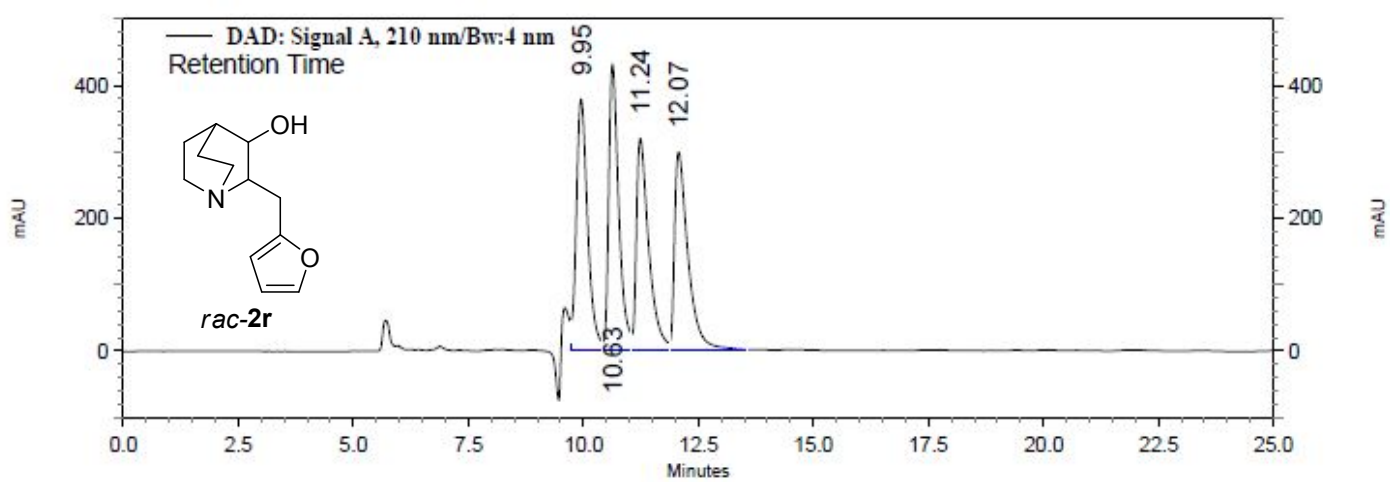

DAD: Signal A,

$210 \mathrm{~nm} / \mathrm{Bw}: 4 \mathrm{~nm}$ Results

\begin{tabular}{ccccccc|}
$\begin{array}{c}\text { Pk } \# \\
\text { P T }\end{array}$ & Area & $\begin{array}{c}\text { Area } \\
\text { Percent }\end{array}$ & Height & $\begin{array}{c}\text { Theoretical } \\
\text { plates (USP) }\end{array}$ & $\begin{array}{c}\text { Resolution } \\
\text { (USP) }\end{array}$ \\
\hline $\mathbf{1}$ & 9.95 & 13395234 & 25.774 & 790691 & 8064 & 0.00 \\
$\mathbf{2}$ & 10.63 & 13558982 & 26.089 & 900359 & 11943 & 1.63 \\
$\mathbf{3}$ & 11.24 & 12343526 & 23.751 & 666753 & 9039 & 1.41 \\
$\mathbf{4}$ & 12.07 & 12673800 & 24.386 & 624171 & 9107 & 1.70 \\
\hline \multicolumn{7}{c}{51971542} \\
\hline
\end{tabular}

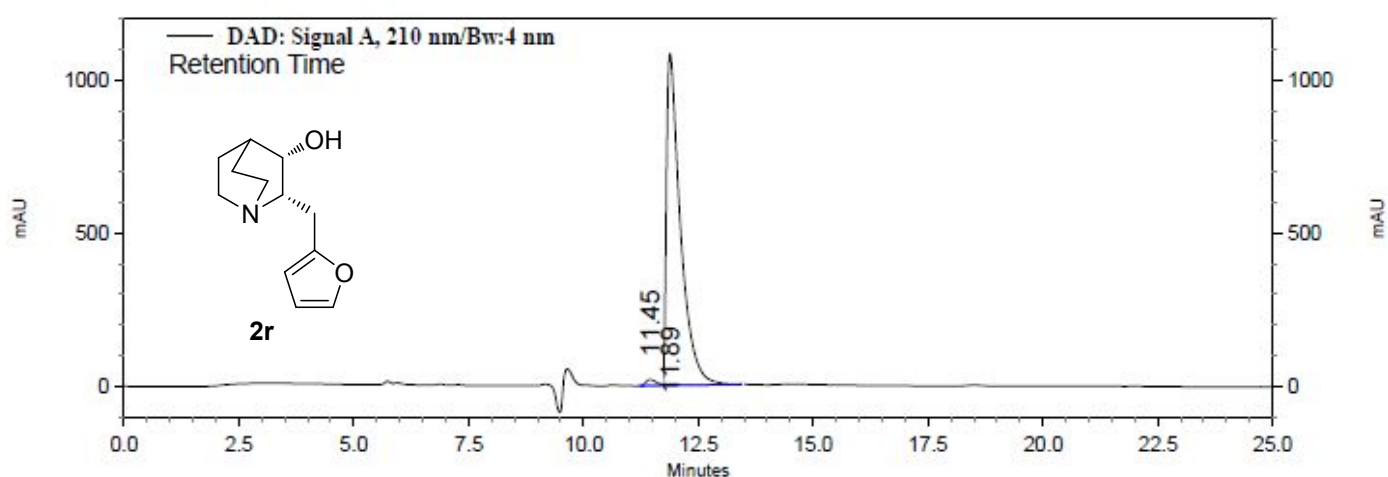

DAD: Signal A,

$210 \mathrm{~nm} / \mathrm{Bw}: 4 \mathrm{~nm}$

\begin{tabular}{ccccccc}
$\begin{array}{c}\text { Results } \\
\text { Pk \# }\end{array}$ & R T & Area & $\begin{array}{c}\text { Area } \\
\text { Percent }\end{array}$ & Height & $\begin{array}{c}\text { Theoretical } \\
\text { plates (USP) }\end{array}$ & $\begin{array}{c}\text { Resolution } \\
\text { (USP) }\end{array}$ \\
\hline $\mathbf{l}$ & 11.45 & 653272 & 1.340 & 42285 & 11333 & 0.00 \\
$\mathbf{2}$ & 11.89 & 48084881 & 98.660 & 2272351 & 8106 & 0.90
\end{tabular}

Totals 


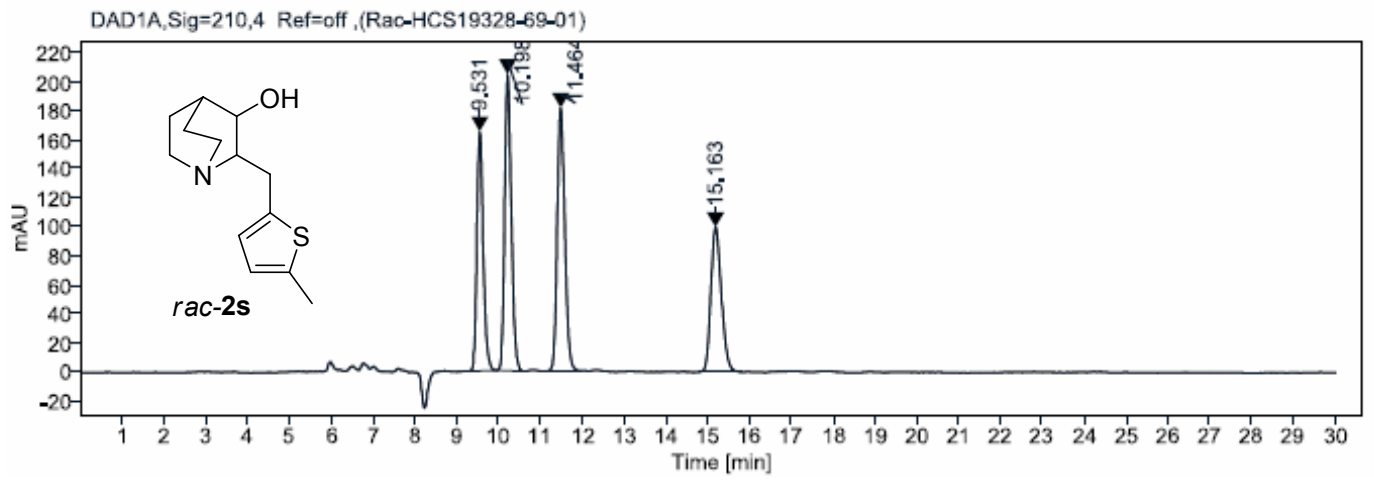

Signal: $\quad$ DAD1A,Sig=210,4 Ref $=$ off

$\begin{array}{ccccccc}\text { RT [min] } & \text { Height } & \text { Area } & \text { Area \% } & \begin{array}{c}\text { Resolution } \\ \text { USP }\end{array} & \text { Tail } & \begin{array}{c}\text { Peak Theoretical } \\ \text { Plates USP }\end{array} \\ 9.53 & 165.099 & 1735.124 & 21.63 & & 1.3 & 19571,37226 \\ 10,20 & 204,655 & 2263,390 & 28,22 & 2,4 & 1,2 & 19871,72198 \\ 11.46 & 181.598 & 2276.103 & 28.37 & 4.1 & 1.2 & 19485.97001 \\ 15,16 & 99,871 & 1746,987 & 21,78 & 9,3 & 1,2 & 17058,00773\end{array}$

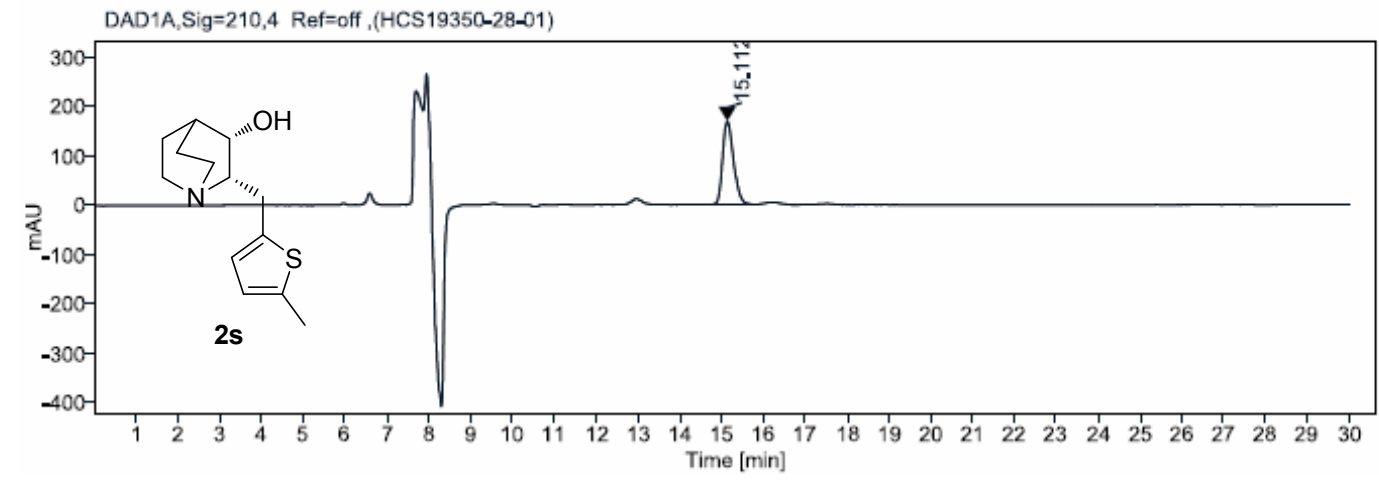

Signal: $\quad$ DAD1A,Sig $=210,4$ Ref $=$ off

$\begin{array}{ccccccc}\text { RT [min] } & \text { Height } & \text { Area } & \text { Area \% } & \begin{array}{c}\text { Resolution } \\ \text { USP }\end{array} & \text { Tail } & \begin{array}{c}\text { Peak Theoretical } \\ \text { Plates USP }\end{array} \\ 15.11 & 167.766 & 3046.815 & 100.00 & & 1.3 & 15689.14226\end{array}$




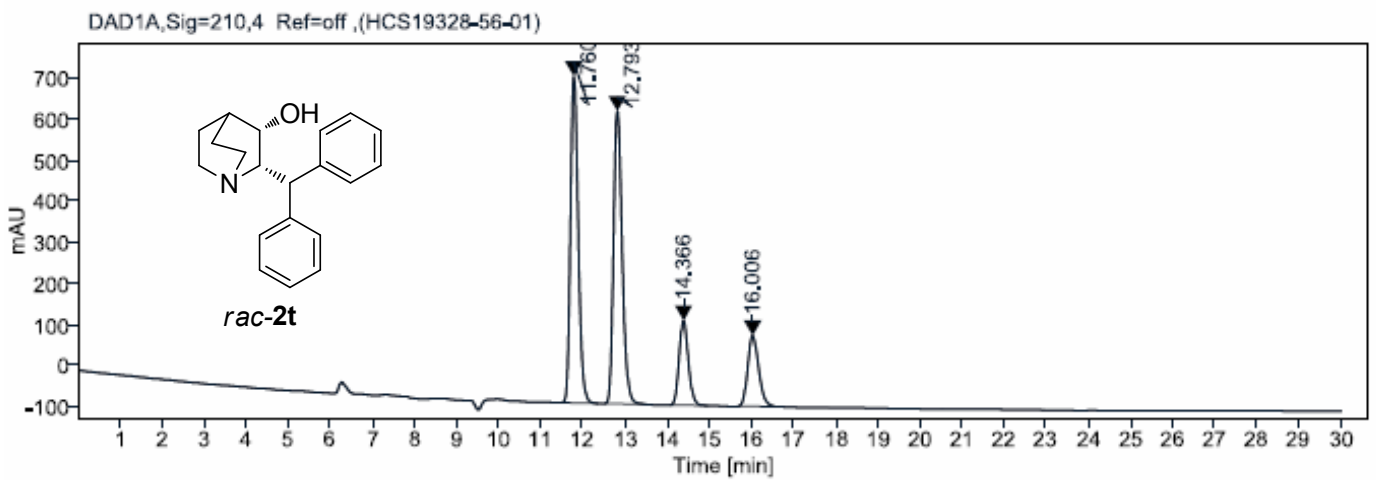

Signal: $\quad$ DAD1A,Sig=210,4 Ref=off

$\begin{array}{ccccccc}\text { RT [min] } & \text { Height } & \text { Area } & \text { Area \% } & \begin{array}{c}\text { Resolution } \\ \text { USP }\end{array} & \text { Tail } & \begin{array}{c}\text { Peak Theoretical } \\ \text { Plates USP }\end{array} \\ 11.76 & 792.729 & 10057.558 & 38.11 & & 1.2 & 20152.33869 \\ 12,79 & 710,934 & 10076,429 & 38,18 & 2,9 & 1,3 & 18989,20529 \\ 14.37 & 205.140 & 3141.757 & 11.90 & 4.1 & 1.1 & 20198.97114 \\ 16,01 & 171,570 & 3114,683 & 11,80 & 3,7 & 1,2 & 17783,60897\end{array}$

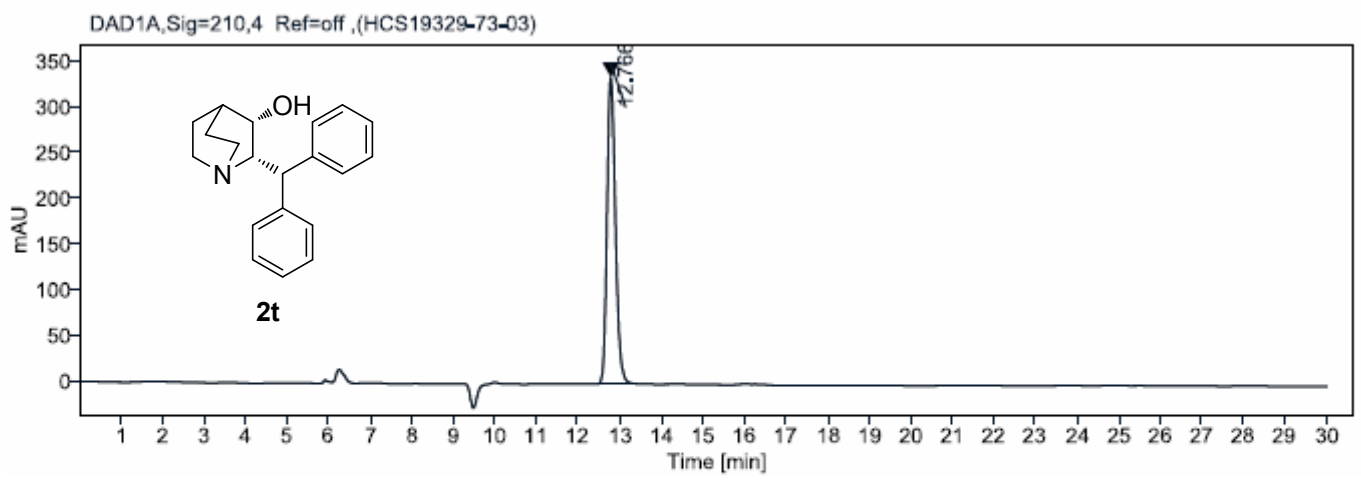

Signal: $\quad$ DAD1A,Sig=210,4 Ref=off

$\begin{array}{ccccccc}\text { RT [min] } & \text { Height } & \text { Area } & \text { Area \% } & \begin{array}{c}\text { Resolution } \\ \text { USP }\end{array} & \text { Tail } & \begin{array}{c}\text { Peak Theoretical } \\ \text { Plates USP }\end{array} \\ 12.77 & 335.345 & 4669.602 & 100.00 & & 1.2 & 19458.81111\end{array}$




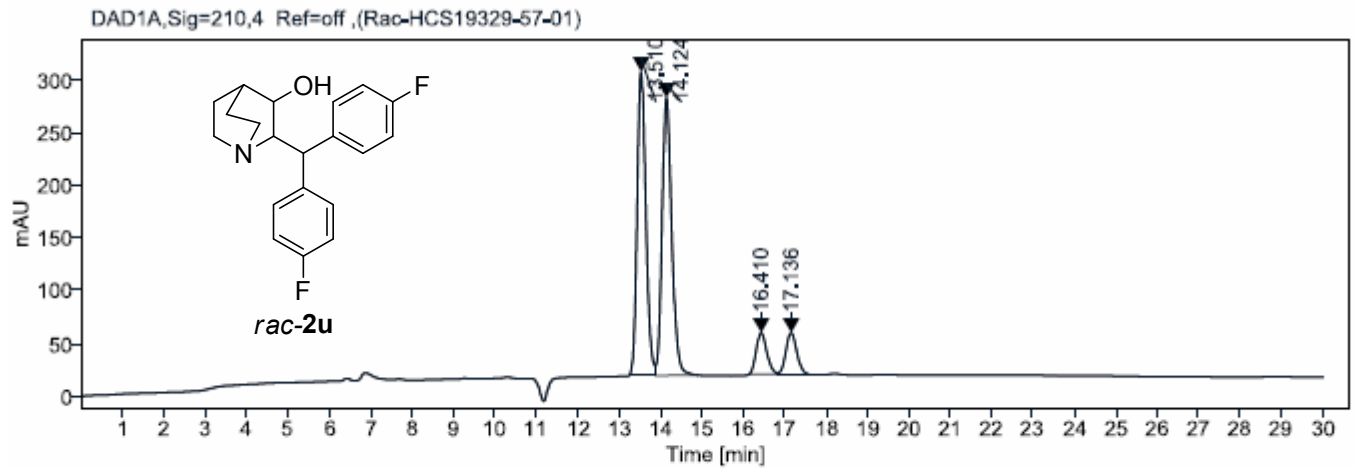

Signal: $\quad$ DAD1A,Sig=210,4 Ref=off

$\begin{array}{ccccccc}\text { RT [min] } & \text { Height } & \text { Area } & \text { Area \% } & \begin{array}{c}\text { Resolution } \\ \text { USP }\end{array} & \text { Tail } & \begin{array}{c}\text { Peak Theoretical } \\ \text { Plates USP }\end{array} \\ 13.51 & 287.926 & 4208.598 & 42.40 & & 1.2 & 19557.42788 \\ 14.12 & 263,148 & 4275,355 & 43,07 & 1,5 & 1,2 & 17905,68421 \\ 16.41 & 39.339 & 705.458 & 7.11 & 5.1 & 1.2 & 18679.62040 \\ 17.14 & 39,499 & 736,154 & 7.42 & 1,5 & 1,1 & 19270,93915\end{array}$

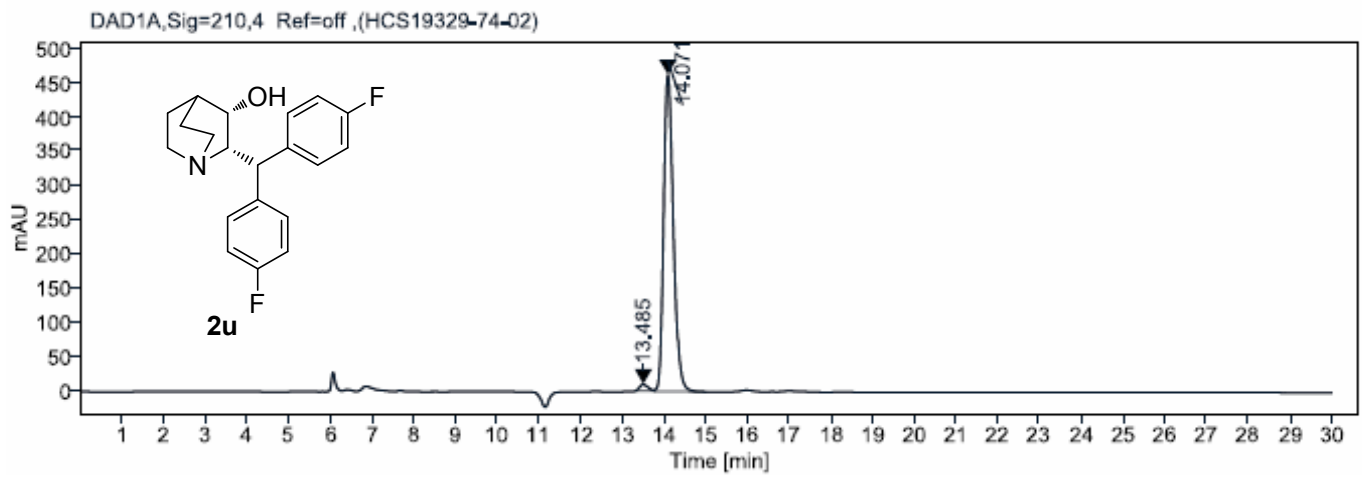

\begin{tabular}{ccccccc} 
Signal: & \multicolumn{2}{c}{ DAD1A,Sig=210,4 Ref=off } & & & & \\
RT [min] & Height & Area & Area $\%$ & $\begin{array}{c}\text { Resolution } \\
\text { USP }\end{array}$ & Tail & $\begin{array}{c}\text { Peak Theoretical } \\
\text { Plates USP }\end{array}$ \\
13.48 & 9.589 & 133.831 & 1.77 & & 1.3 & 20220.25827 \\
14,07 & 462,016 & 7429,476 & 98,23 & 1,5 & 1,3 & 17998,66013
\end{tabular}




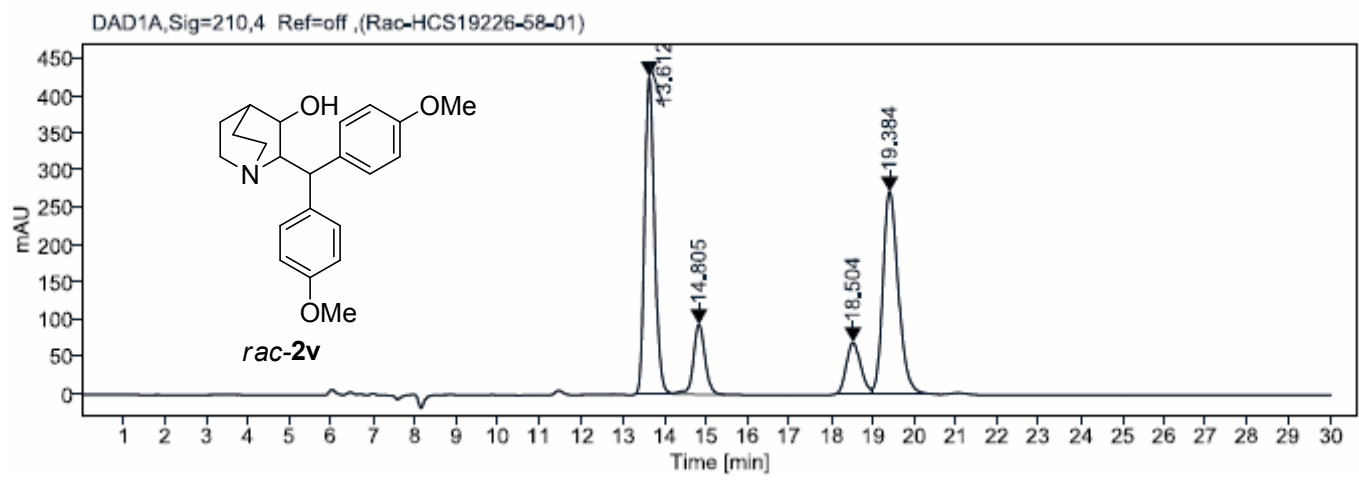

\begin{tabular}{ccccccc} 
Signal: & \multicolumn{2}{c}{ DAD1A,Sig=210,4 Ref=off } & & & & \\
RT [min] & Height & Area & Area \% & $\begin{array}{c}\text { Resolution } \\
\text { USP }\end{array}$ & Tail & $\begin{array}{c}\text { Peak Theoretical } \\
\text { Plates USP }\end{array}$ \\
13.61 & 426.800 & 7006.415 & 40.18 & & 1.2 & 15925.03293 \\
14,80 & 93,732 & 1818,375 & 10,43 & 2,6 & 1,1 & 14295,76576 \\
18.50 & 68.883 & 1654.397 & 9.49 & 6.5 & 1.2 & 13065.49407 \\
19,38 & 271,444 & 6959,553 & 39,91 & 1,3 & 1,2 & 13359,86318
\end{tabular}

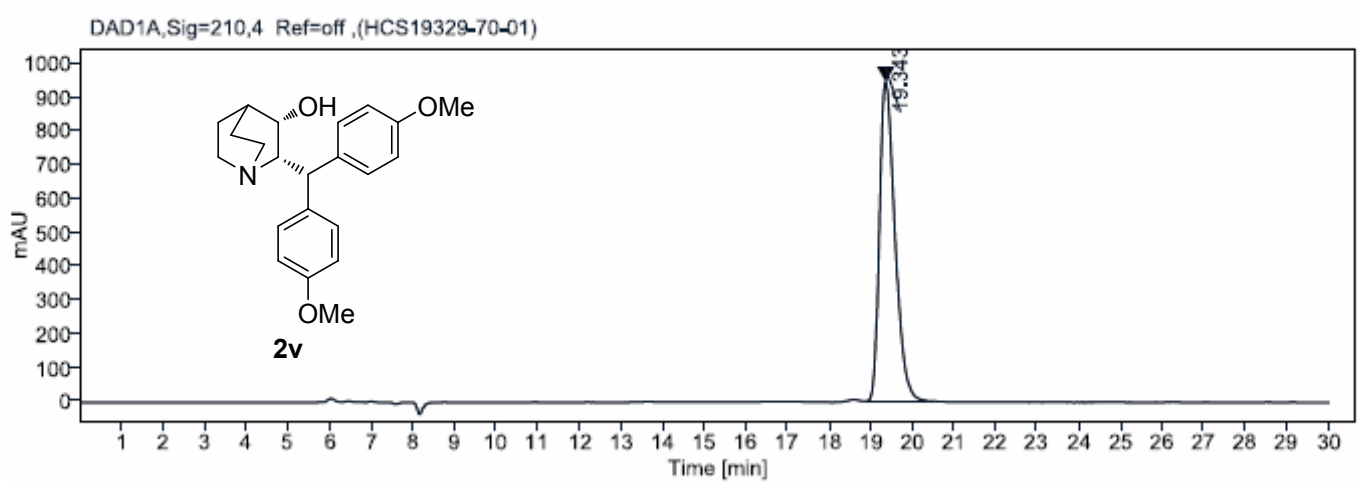

Signal: $\quad$ DAD1A,Sig $=210,4$ Ref $=$ off

$\begin{array}{ccccccc}\text { RT [min] } & \text { Height } & \text { Area } & \text { Area \% } & \begin{array}{c}\text { Resolution } \\ \text { USP }\end{array} & \text { Tail } & \begin{array}{c}\text { Peak Theoretical } \\ \text { Plates USP }\end{array} \\ 19.34 & 942.201 & 24304.662 & 100.00 & & 1.4 & 12933.20228\end{array}$




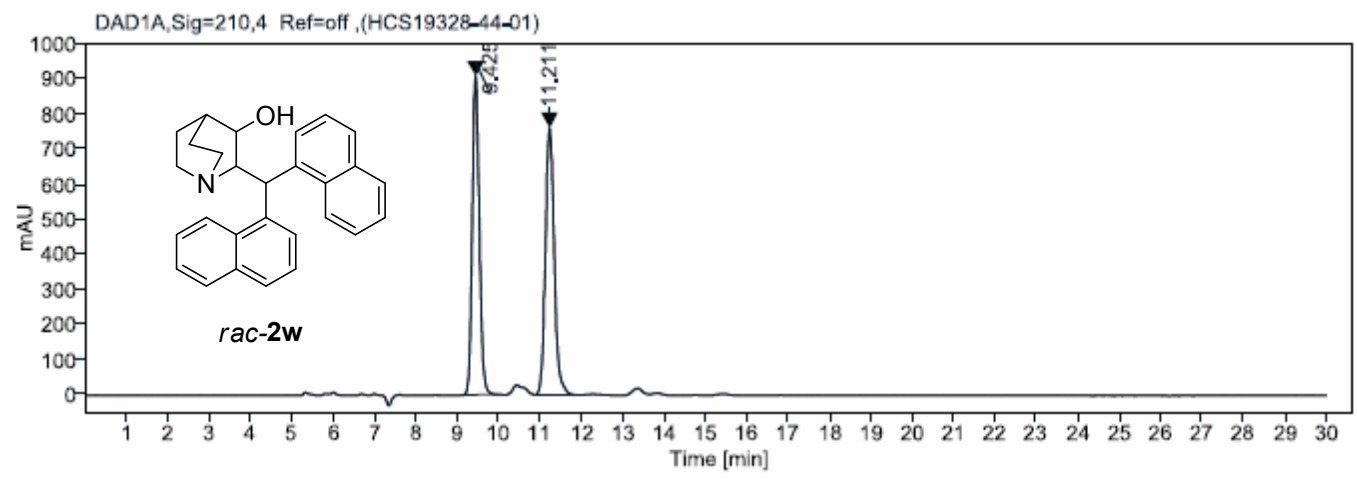

Signal: $\quad D A D 1 A, S i g=210,4$ Ref $=$ off

$\begin{array}{ccccccc}\text { RT [min] } & \text { Height } & \text { Area } & \text { Area \% } & \begin{array}{c}\text { Resolution } \\ \text { USP }\end{array} & \text { Tail } & \begin{array}{c}\text { Peak Theoretical } \\ \text { Plates USP }\end{array} \\ 9.42 & 907.470 & 10793.156 & 49.06 & & 1.1 & 14690.71360 \\ 11,21 & 759,920 & 11207,524 & 50,94 & 5.2 & 1.1 & 13824,77300\end{array}$

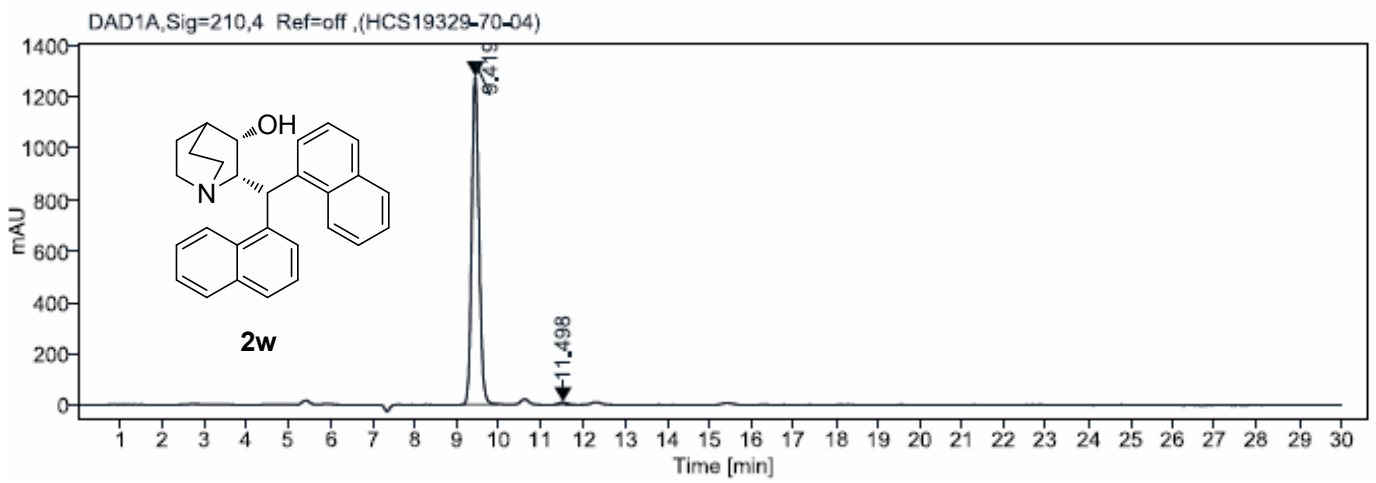

Signal: $\quad$ DAD1A,Sig=210,4 Ref $=$ off

$\begin{array}{ccccccc}\text { RT [min] } & \text { Height } & \text { Area } & \text { Area \% } & \begin{array}{c}\text { Resolution } \\ \text { USP }\end{array} & \text { Tail } & \begin{array}{c}\text { Peak Theoretical } \\ \text { Plates USP }\end{array} \\ 9.42 & 1283.906 & 15511.712 & 99.22 & & 1.1 & 14335.01633 \\ 11,50 & 9,837 & 122,679 & 0,78 & 6,3 & 1.1 & 17933,08853\end{array}$

